\title{
Evaluation of the personal health budget pilot programme
}

Julien Forder, Karen Jones, Caroline

Glendinning, James Caiels, Elizabeth Welch, Kate Baxter, Jacqueline Davidson, Karen

Windle, Annie Irvine, Dominic King and Paul Dolan

November 2012

www.pssru.ac.uk

Discussion Paper 2840_2

Research commissioned by the Department of Health 


\section{Acknowledgements}

This research would not have been possible without the expertise and efforts of a range of people involved with the evaluation of the personal health budgets pilot programme. We would like to thank the project teams in the pilot sites; Accent, our fieldwork collaborators; colleagues from the Department of Health Personal Health Budgets policy and delivery teams; and those people working alongside us in our respective universities. We are also grateful to all those patients and their families who agreed to participate in the study.

We would like to specifically thank a number of our colleagues from PSSRU, at the University of Kent for administrative, technical and research support. During the study period, invaluable support was offered by Jane Dennett, Clara Heath, Edward Ludlow, Amanda Burns, Emily Knapp, Sinead Rider and Lesley Cox.

Furthermore, we would like to thank Teresa Frank from SPRU, at the University of York for her administrative support during the study period.

The research is funded by the Department of Health. However, any views expressed in the report are those of the research team alone. 


\section{Contents}

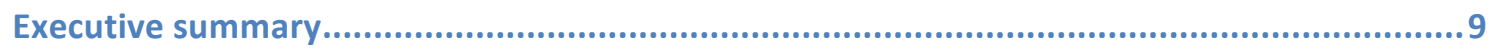

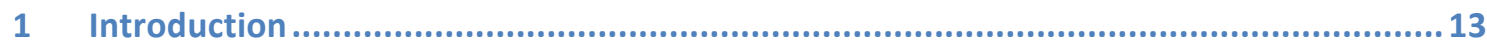

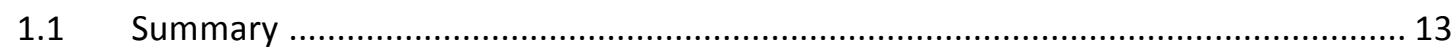

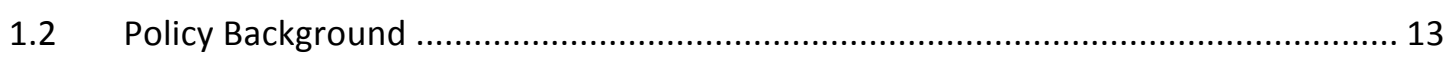

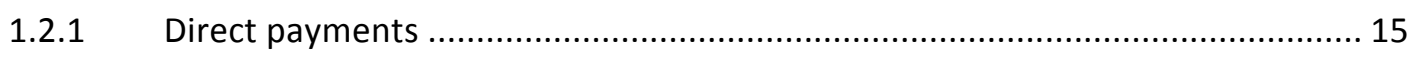

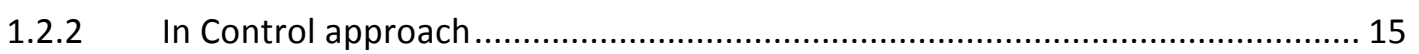

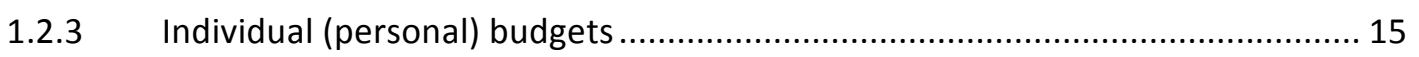

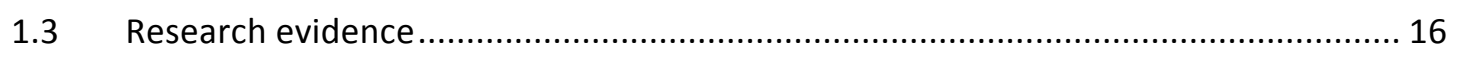

1.3.1 Evaluation of the personal health budget pilot programme .............................. 17

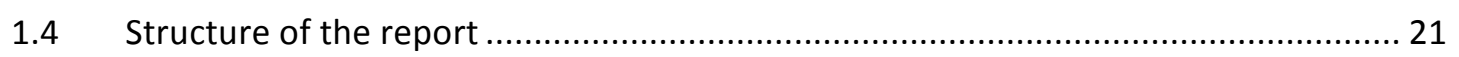

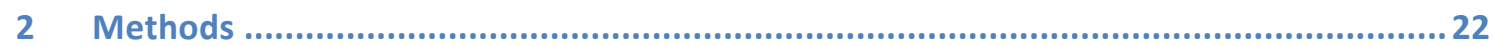

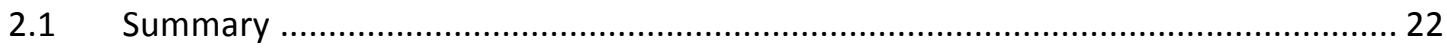

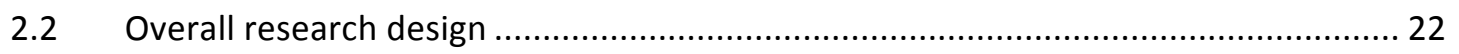

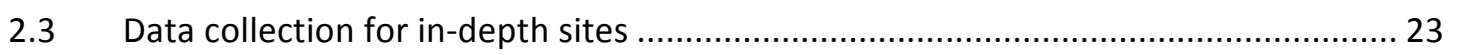

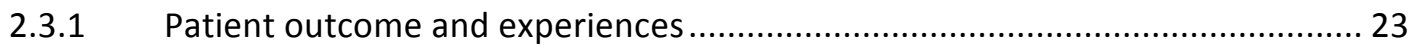

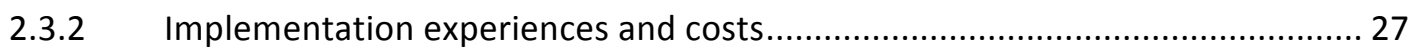

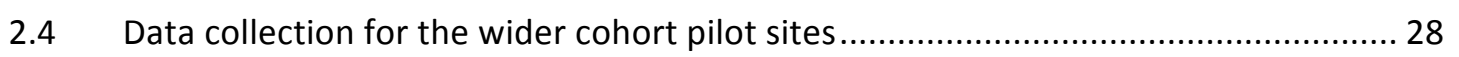

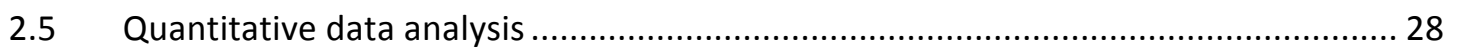

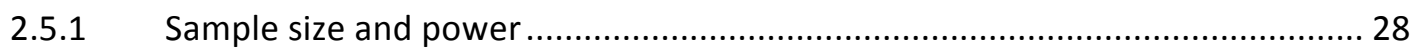

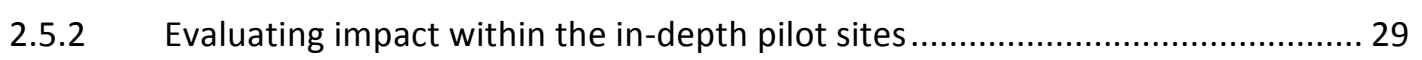

2.5.3 Confounding factors on outcome indicator changes........................................ 32

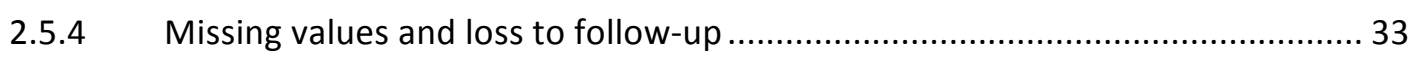

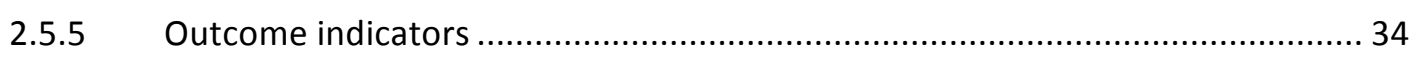

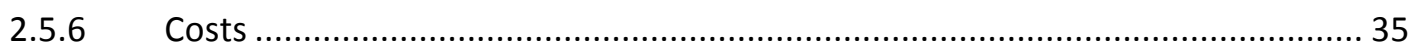

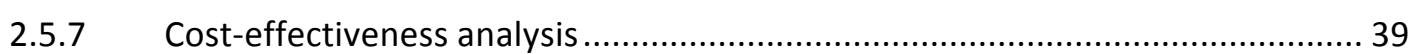

2.5.8 Outcomes, cost-effectiveness and statistical error ............................................. 40

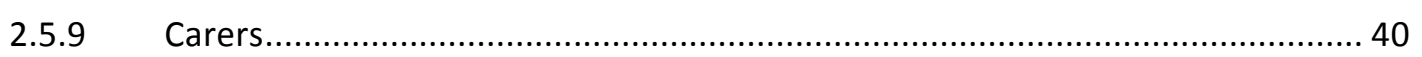

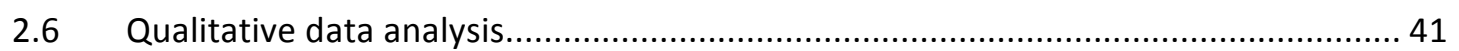

2.7 Challenges to implementing the evaluation design ................................................ 41

2.7.1 Demands on organisational representatives working within the pilot sites ...... 41

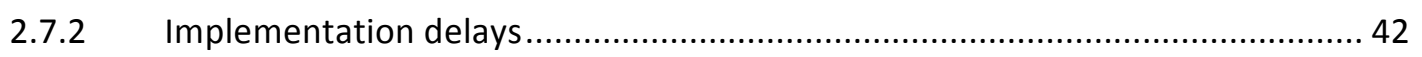

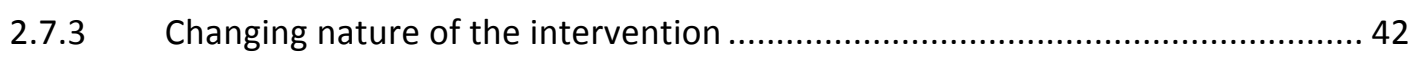

2.7.4 Changes to the policy during the evaluation period........................................... 42

2.8 Research ethics and the PHBE steering group......................................................... 43

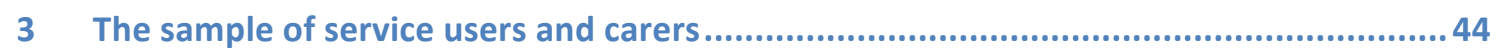

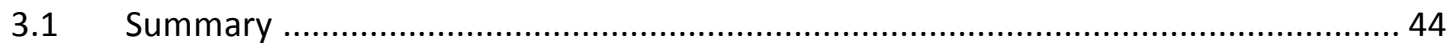

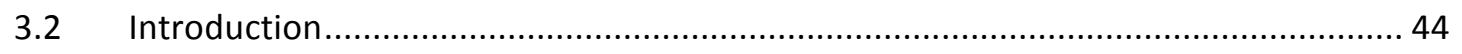

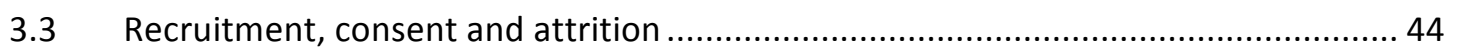




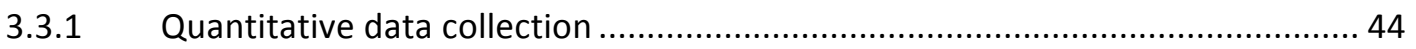

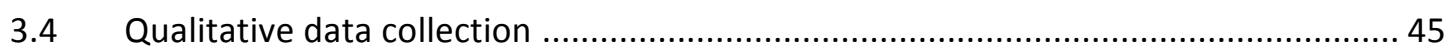

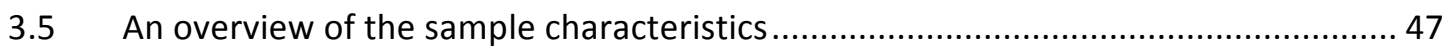

3.5.1 Completed main outcome questionnaires by health condition ........................ 47

3.6 An overview of the sample characteristics ............................................................... 47

3.6.1 Completed baseline outcome questionnaire by age, gender and ethnicity ....... 48

3.6.2 Household composition and informal carers ..................................................... 49

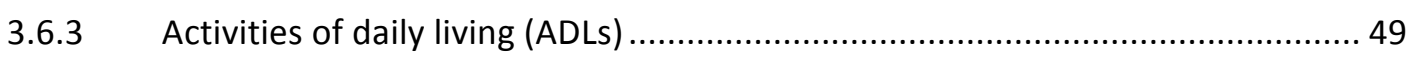

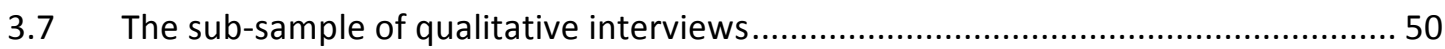

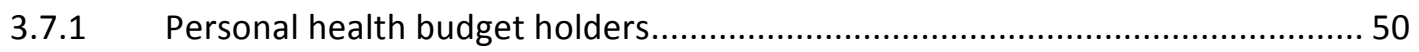

3.7.2 Carers of personal health budget holders ........................................................ 51

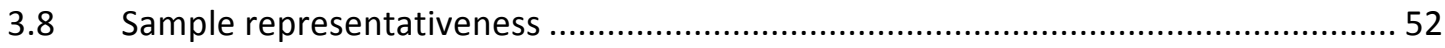

4 The content of the personal health budget support/care plans ............................. 53

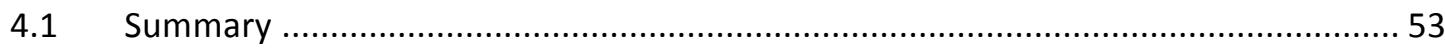

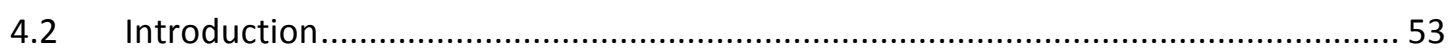

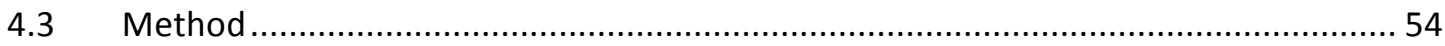

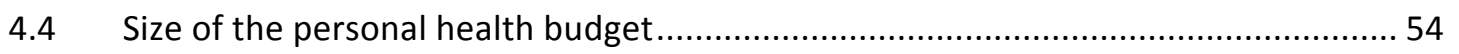

4.5 Personal health budget deployment mechanisms ................................................... 56

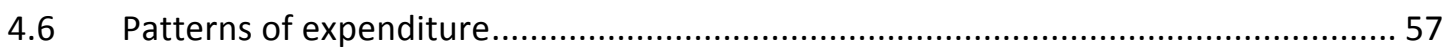

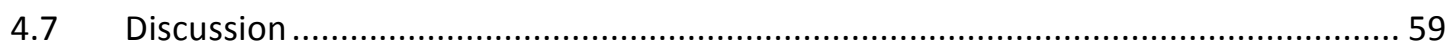

5 The impact of receiving personal health budgets on outcomes..............................6 60

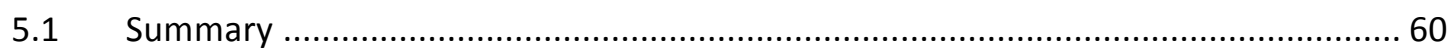

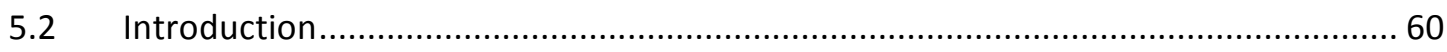

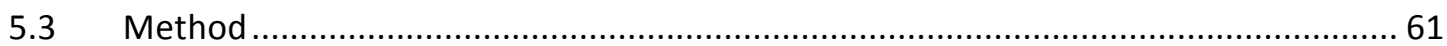

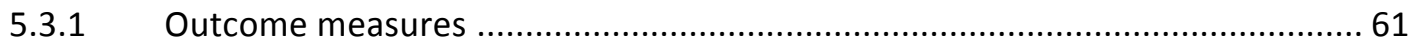

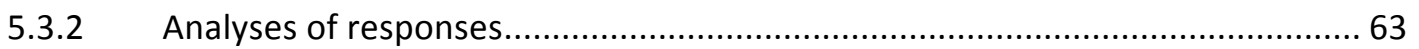

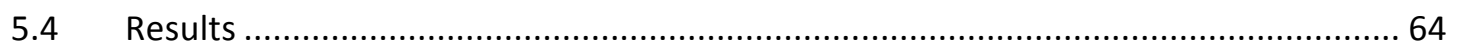

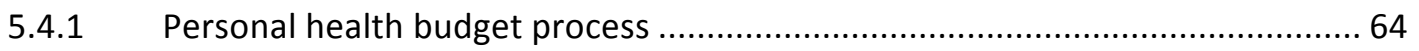

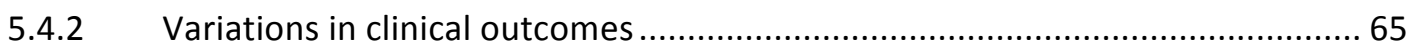

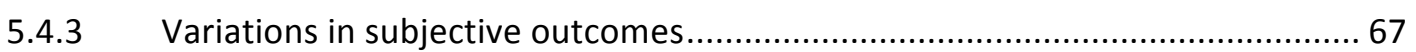

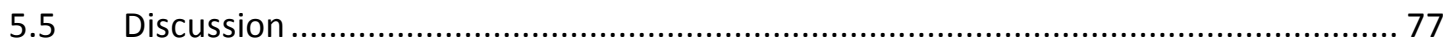

6 Costs and cost effectiveness ............................................................... 79

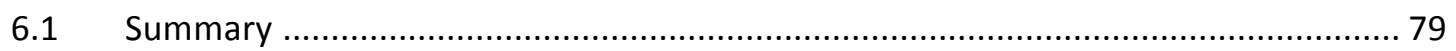

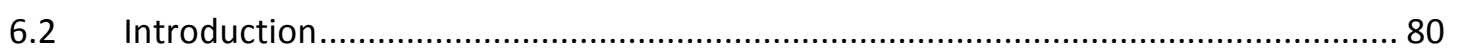

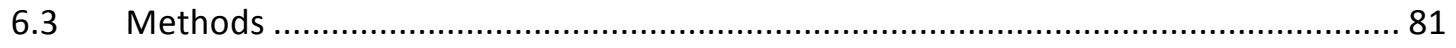

6.3.1 The expected impact of personal health budgets on costs and benefits ........... 81

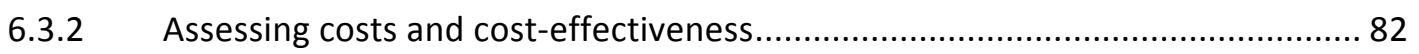

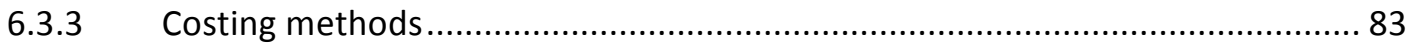

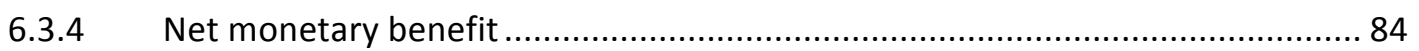

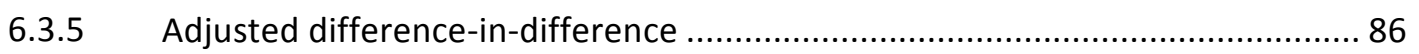

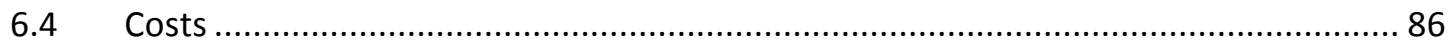




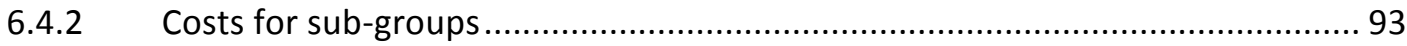

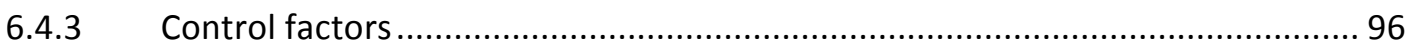

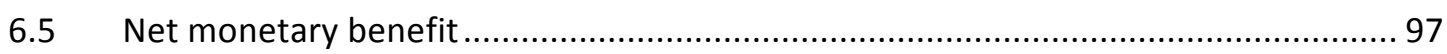

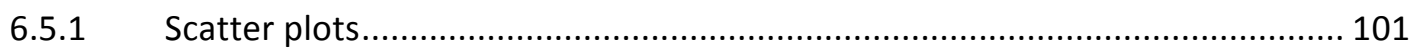

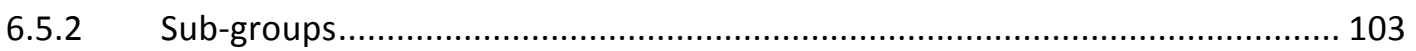

6.5.3 Controlling for baseline characteristics on trend ...................................... 113

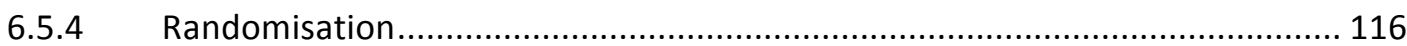

6.6 Exploring the relationship between costs and benefits .......................................... 117

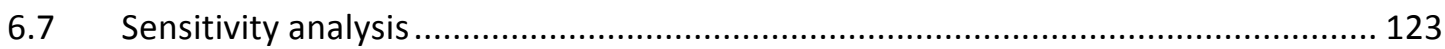

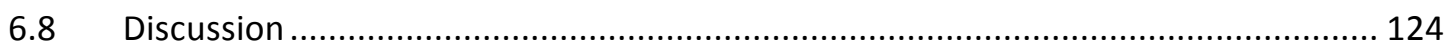

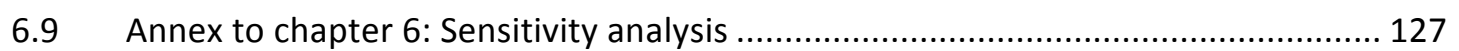

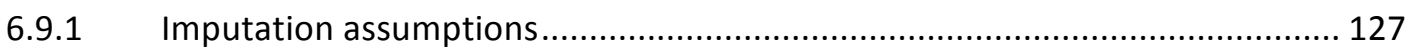

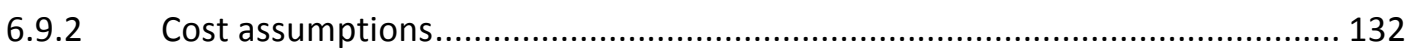

7 The impact of receiving personal health budgets on carers .................................. 135

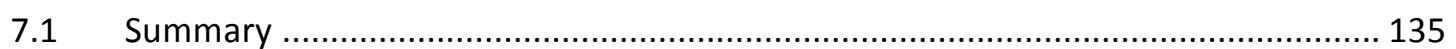

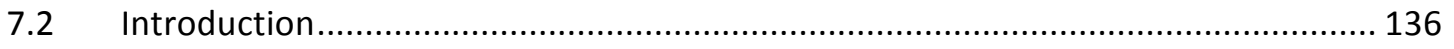

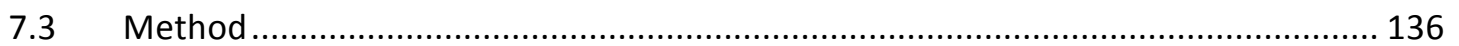

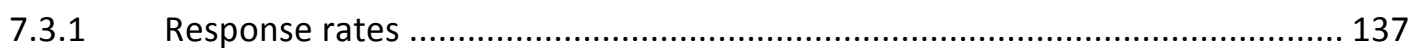

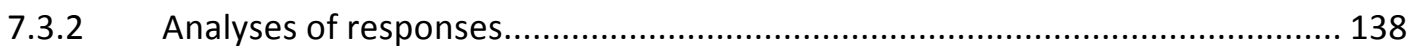

7.4 Variation in informal care receipt between baseline and follow-up .......................... 138

7.5 The impact of personal health budgets on carers ................................................. 140

7.5.1 Impact of personal health budgets on the health and quality of life of carers 143

7.5.2 Outcomes of personal health budgets for carers ........................................... 143

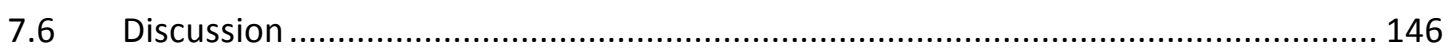

8 Personal health budgets - successes and challenges ....................................... 148

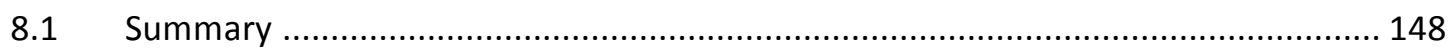

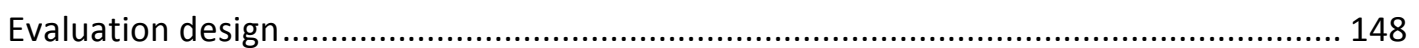

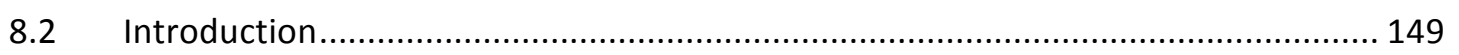

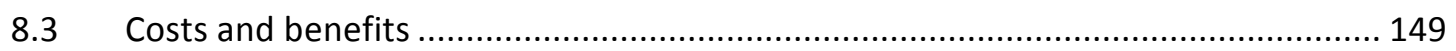

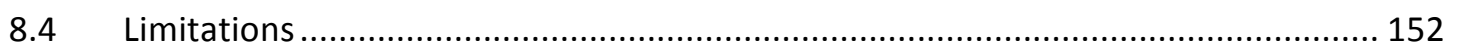

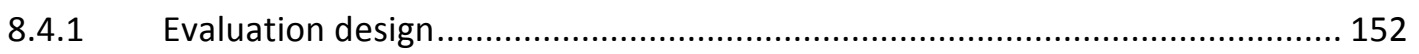

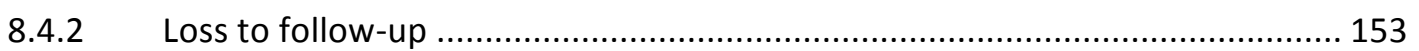

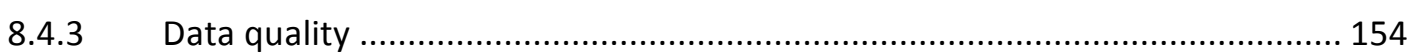

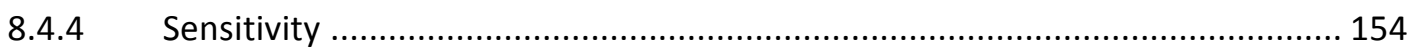

9 Implementing personal health budgets ..................................................... 155

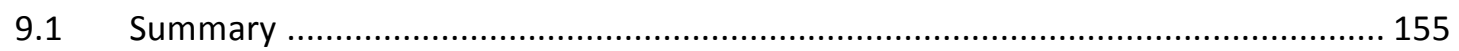

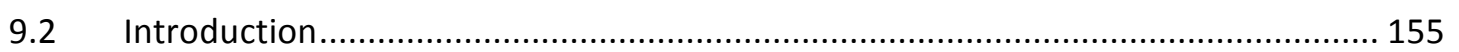

9.3 Optimal use and design of personal health budgets ............................................. 156

9.3.1 Should personal health budgets be deployed? ................................................. 156

9.3.2 Refining the implications for targeting of personal health budgets .................. 158

9.4 Configuring personal health budgets from the individuals' perspective................... 162 


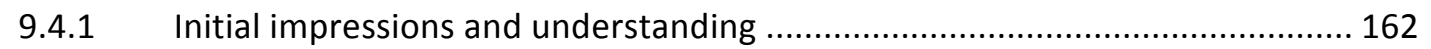

9.4.2 The level of the personal health budget.......................................................... 162

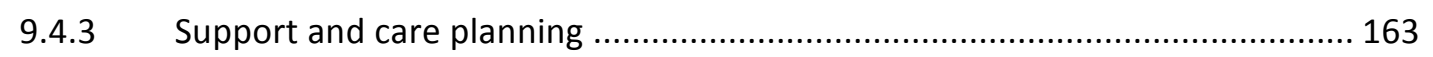

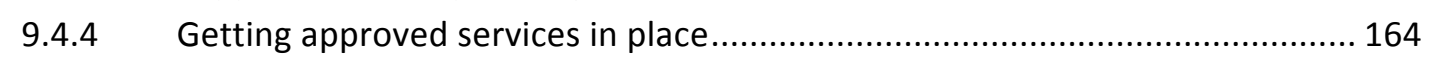

9.4.5 Personal health budgets and social care support............................................ 165

9.4.6 Budget holders' and carers' experiences of deployment options .................... 165

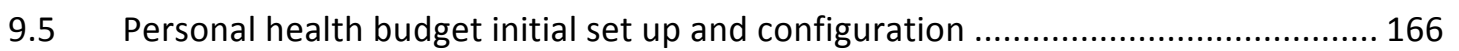

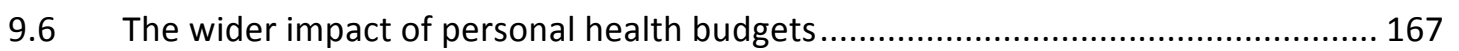

9.6.1 Impacts beyond the individual/family ......................................................... 168

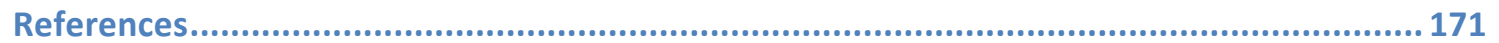

\section{Tables}

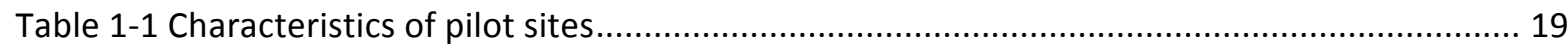

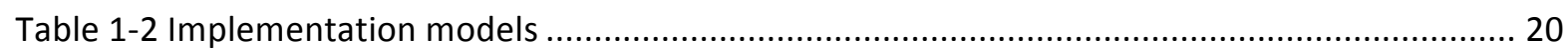

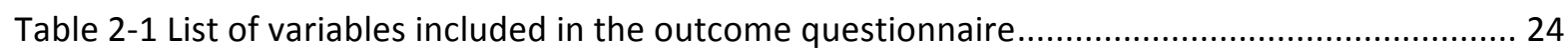

Table 2-2. Services and support - by availability and personal health budget coverage ................... 36

Table 3-1 Completed main outcome questionnaires by health condition ...................................... 47

Table 3-2 Completed baseline outcome questionnaire by age, gender and ethnicity ....................... 48

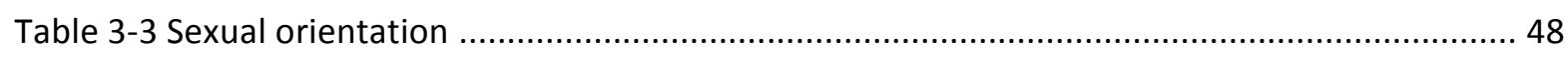

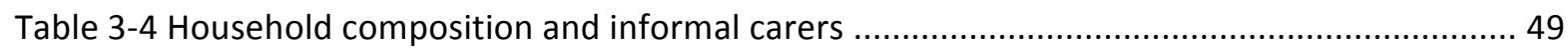

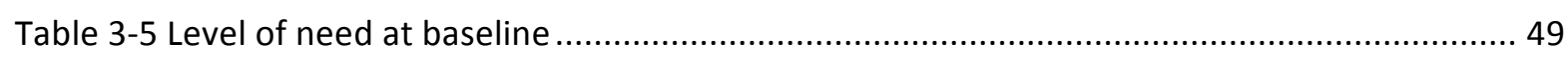

Table 3-6 Number of budget holders interviewed by condition group ............................................ 50

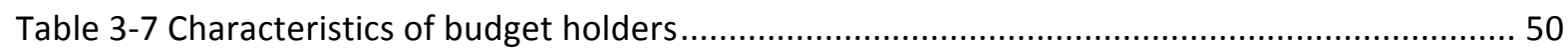

Table 3-8 Length of time personal health budget in place ................................................................. 51

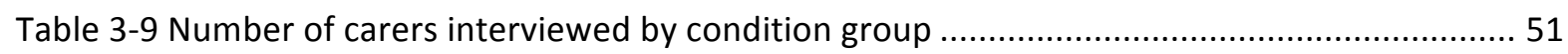

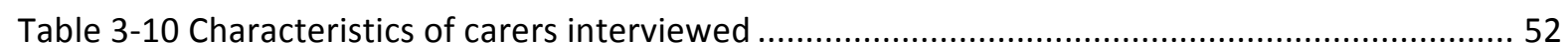

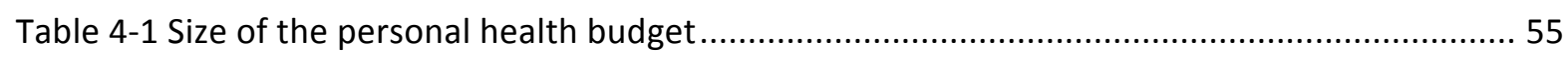

Table 4-2: Average personal health budget by health condition, demographic and socio-economic

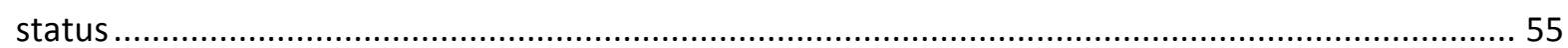

Table 4-3: Description of deployment options by health condition ............................................. 56

Table 4-4: Overall patterns of expenditure funded in personal health budgets, whole PHB sample 57

Table 4-5: Overall patterns of expenditure with the personal health budgets .................................. 58

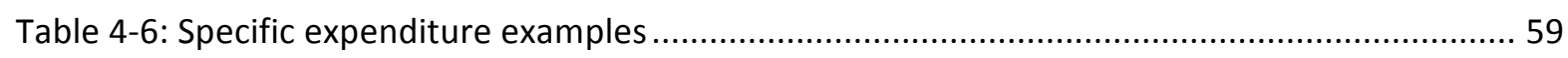

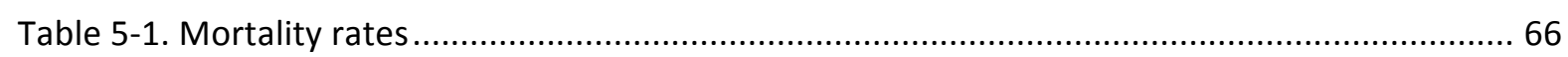

Table 5-2. Change in clinical outcomes between baseline and follow-up............................................ 67

Table 5-3 Outcome measures - means and differences, baseline and follow-up...............................68

Table 5-4 ASCOT and EQ-5D outcome Difference-in-Difference, personal health budget group, with

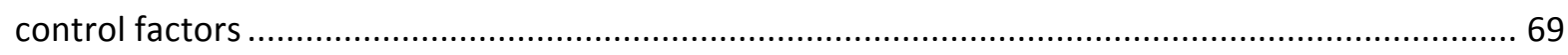

Table 5-5. GHQ-12 and Subjective well-being Difference-in-Difference, personal health budget

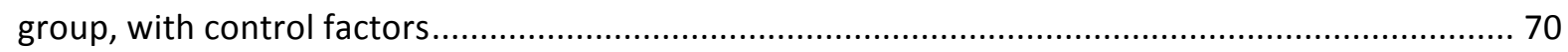

Table 5-6. Outcome measures - impact of personal health budgets, by health condition ............... 72 
Table 5-7.Outcome measures - impact of personal health budgets, by socio-demographic and socio-

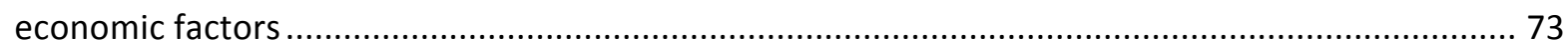

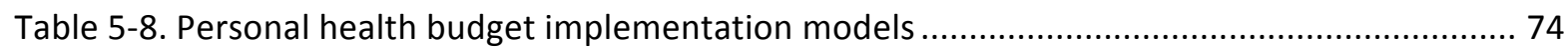

Table 5-9.Outcome measures - impact of personal health budgets, by implementation model...... 75

Table 5-10. Outcome measures - impact of personal health budgets, by budget-amount............... 77

Table 6-1. Service and support costs, by type - Personal health budget group, whole sample ........ 87

Table 6-2. Service and support costs, by type - Control group, whole sample................................. 87

Table 6-3. Differences in service and support costs, by type - whole sample ................................... 91

Table 6-4. Changes in indirect cost between baseline and follow-up, by patient group ................... 94

Table 6-5. Changes in direct cost between baseline and follow-up, by patient group ....................... 94

Table 6-6. Changes in total cost between baseline and follow-up, by patient group .......................... 94

Table 6-7. Differences in service and support costs, by type - over-75 sample ................................ 95

Table 6-8. Differences in service and support costs, by personal health budget type....................... 96

Table 6-9. Difference in mean NMB-change for ASCOT, whole sample, various CE thresholds ........ 98

Table 6-10. Difference in mean NMB-change for EQ-5D, whole sample, various CE thresholds ....... 98

Table 6-11. Statistical significance using bootstrap results, ASCOT and EQ-5D, whole sample

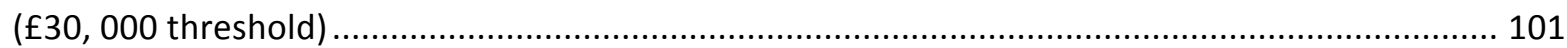

Table 6-12. NMB Diff-in-Diff, by health condition, point estimate and significance probability..... 105

Table 6-13. NMB Diff-in-Diff, by age group, point estimate and significance probability................ 108

Table 6-14. NMB Diff-in-Diff, by personal health budget process, point estimate and significance

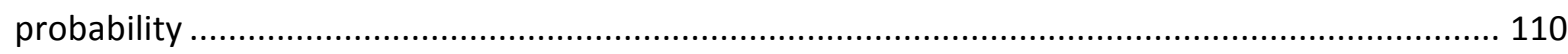

Table 6-15. ASCOT NMB Diff-in-Diff models with control factors - base, personal health budget size

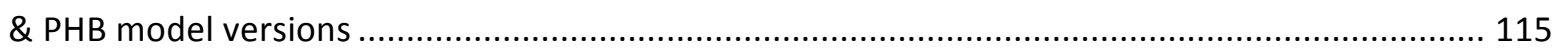

Table 6-16. ASCOT NMB Diff-in-Diff models with control factors - base, personal health budget size

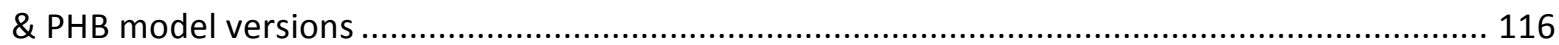

Table 6-17. Differences in ASCOT score - controlling for expenditure .......................................... 121

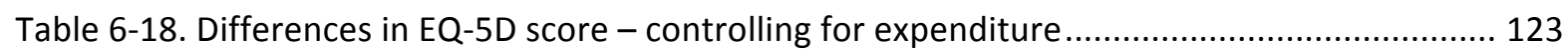

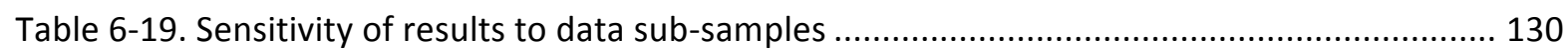

Table 7-1 Informal care receipt-sample means and differences, baseline and follow-up.............. 138

Table 7-2 Direction of change in receipt of informal care, with control factors ............................. 139

Table 7-3 Direction of change in receipt of informal care, with implementation models ............... 140

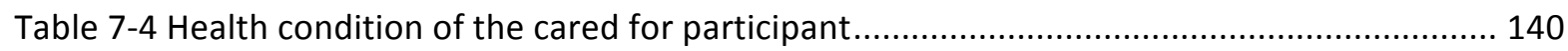

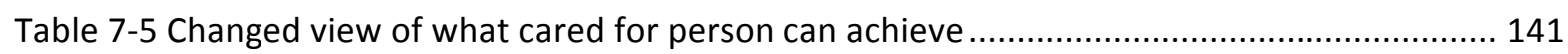

Table 7-6 Satisfaction and support planning process (personal health budget group)..................... 141

Table 7-7 Caring role and carers' health in the last 12 months.................................................... 143

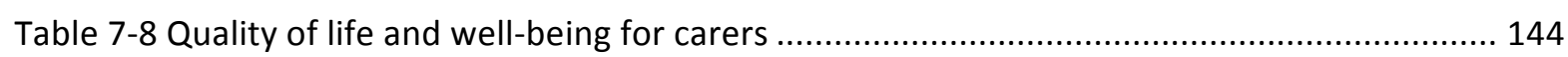

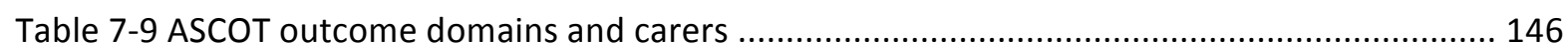

\section{Figures}

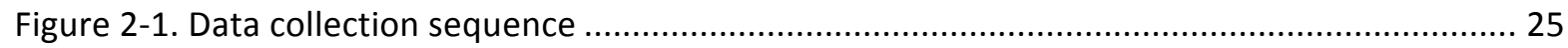

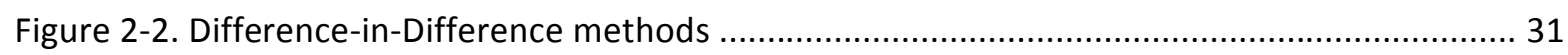


Figure 3-1. The sample.

Figure 6-1. The impact of personal health budgets on costs and benefits ....................................... 82

Figure 6-2. Density plot - distribution of total costs, all participants ............................................ 89

Figure 6-3. Changes in costs and differences - personal health budget and control groups, whole sample

Figure 6-4. ASCOT and EQ-5D NMB Diff-in-Diff, whole sample, point estimates and confidence

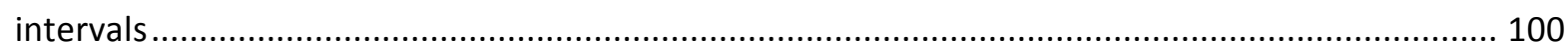

Figure 6-5. Cost-effectiveness plot - ASCOT outcome, whole sample .............................................. 102

Figure 6-6. Cost-effectiveness plot - EQ-5D outcome, whole sample .......................................... 103

Figure 6-7. NMB Diff-in-Diff, by health condition, point estimates and confidence intervals ......... 106

Figure 6-8. Cost-effectiveness plot - ASCOT outcome, NHS Continuing Healthcare sub-sample .... 107

Figure 6-9. Cost-effectiveness plot - EQ-5D outcome, NHS Continuing Healthcare sub-sample .... 107

Figure 6-10. NMB Diff-in-Diff, by age group, point estimates and confidence intervals................. 109

Figure 6-11. NMB Diff-in-Diff, by personal health budget process, point estimates and confidence

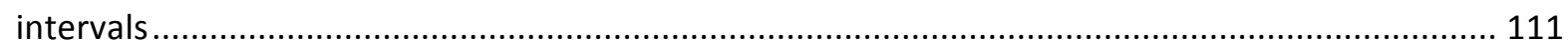

Figure 6-12. Cost-effectiveness plot - ASCOT outcome, personal health budget model 1............. 112

Figure 6-13. Cost-effectiveness plot - EQ-5D outcome, personal health budget model 1 ............. 112

Figure 6-14. ASCOT NMB Diff-in-Diff, comparing base MI and sensitivity MI results, point estimates

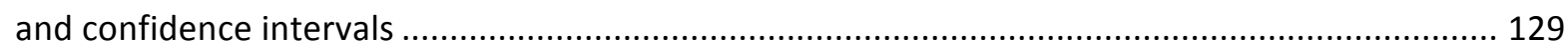

Figure 6-15. EQ-5D NMB Diff-in-Diff, comparing base MI and sensitivity MI results, point estimates

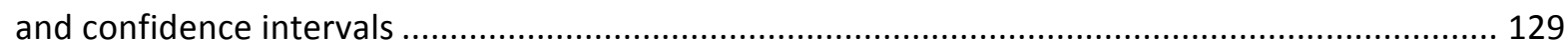

Figure 6-16. ASCOT NMB Diff-in-Diff, comparing MI models (truncated regression is base against

$P M M)$, point estimates and confidence intervals

Figure 6-17. EQ-5D NMB Diff-in-Diff, comparing MI models (truncated regression is base against $P M M)$, point estimates and confidence intervals

Figure 6-18. ASCOT NMB Diff-in-Diff, comparing base cost assumption ( $f 1000$ limit) with the alternative assumption ( $₫ 500$ limit), point estimates and confidence intervals.

Figure 6-19. EQ-5D NMB Diff-in-Diff, comparing base cost assumption ( $f 1000$ limit) with the alternative assumption ( $₫ 500$ limit), point estimates and confidence intervals

Figure 6-20. ASCOT NMB Diff-in-Diff, comparing base cost assumption ( $£ 1000$ limit) with the alternative assumption ( $€ 0$ limit), point estimates and confidence intervals.

Figure 6-21. EQ-5D NMB Diff-in-Diff, comparing base cost assumption ( $f 1000$ limit) with the alternative assumption ( $f 0$ limit), point estimates and confidence intervals.. 


\section{Executive summary}

1. The personal health budget initiative is a key aspect of personalisation across health care services in England. Its aim is to improve patient outcomes, by placing patients at the centre of decisions about their care. Giving people greater choice and control, with patients working alongside health service professionals to develop and execute a care plan, given a known budget, is intended to encourage more responsiveness of the health and care system.

2. The personal health budget programme was launched by the Department of Health in 2009 after the publication of the 2008 Next Stage Review. An independent evaluation was commissioned alongside the pilot programme with the aim of identifying whether personal health budgets ensured better health and care outcomes when compared to conventional service delivery and, if so, the best way for personal health budgets to be implemented.

\section{Study design and methodology}

3. The evaluation took a longitudinal approach and included people with any of six conditions: chronic obstructive pulmonary disease, diabetes and long-term neurological conditions; mental health; stroke; and patients eligible for NHS Continuing Healthcare.

4. Of the 64 sites in the personal health budgets pilot at onset, 20 sites were selected to be in-depth evaluation sites, with the remainder being wider cohort sites. Sites implemented personal health budgets in different ways, varying the choice people had about what services could be purchased with the budget, flexibility as to how the budget could be managed, how explicit the budget size was and how the size of the budget was calculated.

5. The evaluation used a controlled trial to compare the experiences of people selected to receive personal health budgets with those of people continuing with conventional support arrangements. A pragmatic design was used. After applying initial selection criteria, in some sites people were randomised into the personal health budget group or a control group. In other sites, the personal health budget group was recruited from patients of those health care professionals in the pilot offering budgets and a control group from patients of non-participating health care professionals.

6. The evaluation followed a mixed design, using both quantitative and qualitative methodologies to explore patient outcomes, experiences, service use and costs. Just over 1000 individuals were recruited into each arm of the study in order to give the analysis sufficient statistical power. The study used methods to deal with a range of issues, including: attribution of effect (addressed using a difference-indifference approach and testing with confounding factors); missing data (multiple imputation); allocation of costs (a costing model); and statistical inference regarding the significance of sample results (parametric and bootstrap estimators, powered sample sizes). Limitations with respect to these methods should be noted.

7. Cost effectiveness was assessed by estimating whether the personal health budgets group experienced greater benefits than the control group who received conventional service delivery, after netting off the difference in service and support costs between the groups. Net benefits were expressed in monetary terms for this purpose, using willingness-to-pay thresholds. The groups were compared at a target 12-month after initial recruitment, with any baseline differences (in net benefit levels) between the groups subtracted from the follow-up difference to reduce attribution bias.

8. The qualitative analysis involved interviews with organisational representatives, personal health budget holders and carers of budget holders. The framework approach was used for the analysis, with the data organised by themes according to the topic guides used in the interviews. 
9. A personal health budget might have an impact on outcomes in three different ways:

- Through the direct benefits of having more choice and control on quality of life;

- From the change in the services and support people fund using their personal health budgets, allowing them to tailor care and support to their own needs and preferences. This process could improve the recipients' health and functioning and, in turn, their quality of life. There might, conversely, be a negative effect if people make ill-informed choices about their care; and

- $\quad$ From any change in the overall level of funding of people's personal health budgets as compared to what they would have received under conventional service arrangements.

10. A range of 'impact' indicators were assessed, including clinical effectiveness measures; mortality rates; care and health-related quality of life measures; psychological health scales and overall wellbeing indicators. Changes in these impact indicators between the personal health budget and control groups indicated the benefits consequences of the initiative.

11. People's use of a wide range of services and support was assessed between the groups, including: community health; therapy and nursing services; social care services aimed at meeting health and care needs; well-being services; and also, primary and secondary services (which might be affected indirectly from the use of a personal health budget). Changes in service expenditure between the personal health budget and control groups indicated the cost consequences of the initiative.

\section{Findings}

12. The main benefit-related implications of personal health budgets were as follows (unless otherwise noted, significance is assessed at the $95 \%$ confidence level):

- The use of personal health budgets was associated with a significant improvement in the carerelated quality of life (ASCOT) and psychological well-being (GHQ-12) of patients (at 90\% confidence).

- Personal health budgets did not appear to have an impact on health status per se over the 12 month follow-up period. No significant effects were found with regard to two clinical measures ( $\mathrm{HbA} 1 \mathrm{C}$ and lung-function tests, used where relevant) and there was no significant difference in mortality rates between the groups. Consistent with these results, the study did not find that personal health budgets had a significant effect on EQ-5D compared to the control group.

13. The configuration of personal health budgets also appeared to be important. Generally, a more positive effect on outcome indicators was seen where sites: choose to be explicit in informing the patients about the budget amount; provided a degree of flexibility as to what services could be purchased; and provided greater choice as to how the budget could be managed. Some negative impacts were found for sites using configurations with less flexibility and choice than other sites.

14. Separating personal health budgets into high-value (i.e. a budget of more than $£ 1,000$ per year) and low-value, it was the former that showed a significant positive impact on care-related quality of life (ASCOT) and psychological well-being (GHQ-12). High-value budgets were more likely for people with greater levels of need or where sites opted to be more inclusive about what budgets could cover.

15. Sub-group analyses for individual patient groups were limited by the relatively small sample sizes for individual groups (hence low statistical power). Nonetheless, a number of effects were strong enough to emerge in these analyses: personal health budgets were associated with improvements regarding ASCOT-measured outcome change (at 90\%), psychological well-being (GHQ-12) and subjective wellbeing, for the COPD cohort. 
16. The main findings of the cost analysis were:

- The cost of inpatient care (an 'indirect' cost) was significantly lower for the personal health budget group compared to the control group after accounting for baseline differences.

- The ('direct') costs of well-being and other health services were both significantly higher for the personal health budget group compared to controls.

- Other categories of direct and indirect cost showed no difference between the groups.

- The difference in direct and indirect total costs between personal health budget and control groups after accounting for baseline differences were not statistically significant.

17. The cost analyses for the individual health condition groups were mostly inconclusive as a result of the modest sub-sample sizes. However, indirect costs were found to be lower for personal health budgetholders in the mental health and NHS Continuing Healthcare sub-groups (at the $90 \%$ confidence level). Total costs were also lower in the group of people with high-value personal health budgets compared to the controls (significant at the $90 \%$ confidence level).

18. The change in the balance of services that budget-holders used also suggested that more of their services were secured from outside conventional NHS providers than the control group.

19. Personal health budgets were assessed to be cost-effective relative to conventional service delivery if they produced greater net benefits than this usual care comparator. Key findings were:

- Using care-related quality of life (ASCOT) measured net benefits, personal health budgets were cost-effective relative to conventional service delivery (at the $90 \%$ confidence level).

- There was no significant difference in the net benefit between the groups using health-related quality of life (EQ-5D) measured benefits.

- Notwithstanding the small sample sizes in the sub-group analyses, personal health budgets showed higher ASCOT-measured net benefits than conventional services for the $\mathrm{CHC}$ and mental health sub-groups (at the $90 \%$ confidence level).

- Personal health budgets implemented following the main ethos of the policy (greater choice and control) were cost-effective at the $95 \%$ confidence level, as were those with high-value budgets.

20. Sensitivity analysis was used to explore whether the main results changed if some of the assumptions in the analysis were altered (regarding missing data imputation and the costing of personal health budgets). These analyses substantiated our main results in almost all cases. With the main alternative assumptions, personal health budgets were cost-effective (with ASCOT-measured benefits) at the 95\% confidence level, rather than the $90 \%$ level.

21. Personal health budgets were cost-effective using the ASCOT quality of life measure. Although this measure was developed originally to assess the consequences of social care services, its focus on carerelated quality of life implications makes it highly relevant for general use with people managing longterm conditions. Other studies have shown that people value care-related quality of life as measured by ASCOT in that they are willing to exchange shorter life expectancy for better ASCOT-measured quality of life. Improvement in ASCOT quality of life was found for people who were not using social care at baseline as well as for those people who were in receipt of these services.

The impact of personal health budgets on the caring role and quality of life

22. Difference-in-difference multivariate analysis revealed no significant differences in the reported use of informal care between personal health budget and control groups overall. 
23. Analysis of a more limited sample of carers of people with personal health budgets suggested that they were more likely to report better quality of life and perceived health than carers of people in the control group. Carers seemed to be satisfied with the personal health budget process in terms of support planning, the amount of the budget and the amount of help that was offered when deciding what services or support to purchase from the personal health budget. The small sample (147) in this case restricted the extent to which confounding factors could be accounted for in this analysis.

\section{User and carer perspectives on PHBs}

24. At around three months after the offer of the personal health budget 58 personal health budget holders were interviewed to discuss their experiences of the process. Fifty-two took part in a follow-up interview nine months after the offer of the personal health budgets. Nineteen carers providing assistance to a personal health budget holder were interviewed at three months after the offer; of which 13 carers were interviewed at nine months after the offer of the budget.

25. At nine months after study recruitment, the majority of budget-holders and carers reported positive impacts of the personal health budget - on their health and well-being, health care and other support arrangements and for other family members. Effect on their use of health services or changes in relationships with health professionals were less likely to be reported. Most interviewees appreciated the increased choice, control and flexibility of the personal health budget, although some thought the benefits were curtailed by restrictions on what the budget could be used for, lack of services and budgets being too small for their needs.

\section{Recommendations for policy and practice}

26. The findings from the study suggest a number of recommendations regarding the possible roll-out of personal health budgets:

- The study concludes that personal health budgets were cost-effective, given the assumptions made (esp. regarding the value of ASCOT quality of life) and thus support a wider roll out.

- High-value personal health budgets were most cost-effective, suggesting that personal health budgets should be initially targeted at people with greater need, to act as substitute for conventional service delivery.

- Personal health budgets were cost-effective for people with mental health problems and those receiving NHS continuing healthcare but the analyses for other health conditions were inconclusive due to small sub-samples sizes.

- The budget-holders that were interviewed emphasised the value of information and guidance from sites about the size and operation of their budgets, including what services were covered.

- The use of personal health budgets is likely to result in greater use of 'non-conventional' providers. Further research is required to better understand the scale of these changes. 


\section{Introduction}

\subsection{Summary}

In England, the personal health budget initiative is an important part of the current personalisation agenda for health care services. Its aim is to place patients at the heart of decisions about services they receive.

The personal health budget initiative was first proposed in the 2008 NHS Next Stage Review as a process of giving patients greater control over services they receive and how their support is managed. A number of design principles underlie personal health budgets, including:

1. Patients should know the resource level available within the budget;

2. Patients should be encouraged to develop a support/care plan that details how the resource will be used to meet their identified needs;

3. Patients should decide how they would like the budget to be managed.

In 2009, the Department of Health invited PCTs to become pilot sites for the personal health budget pilot programme. An evaluation was commissioned to run alongside in order to provide an account of the personal health budget process from the system and from the view of budget holders and carers.

Initially, 64 pilot sites were involved in piloting personal health budgets and contributing to the evaluation. During the evaluation period, the pilot programme remained open and now there are 75 pilot sites implementing personal health budgets.

Twenty sites from across the pilots were selected to be in-depth evaluation sites, with the remainder being wider cohort sites.

The overarching aim of the evaluation was to identify whether personal health budgets ensured better health and care-related outcomes when compared to conventional service delivery and, if so, the best way they should be implemented.

\subsection{Policy Background}

In England, the personal health budget initiative is an important part of the current personalisation agenda for health care services, with the aim of placing patients at the heart of decisions about the care and services they receive (Department of Health, 2009). The aim of the initiative is to encourage the NHS to become more responsive to the needs of patients by providing greater choice (HM Government, 2010b), which could in turn lead to improved efficiency within the NHS. Both aims are high on the political agenda at a time when demand on the health care system is increasing, with greater numbers of people living with one or more complex long-term conditions, and within the current climate of constrained finances. As context for these aims, around 15.4 million people in 
England have at least one long-term health condition, accounting for 70 per cent of the health and social care budget ${ }^{1}$.

The personal health budget initiative was proposed in the 2008 NHS Next Stage Review as a way of giving patients greater choice and control over services they receive and how their support is managed. However, the idea was not new, as in 2009 Glasby and colleagues reported a growing impetus around personalisation within the NHS among academics (Glasby et al., 2009; Glendinning et al., 2008; Leadbeater et al., 2008; Alakeson, 2007; Glasby and Hasler, 2004; Glendinning et al., 2000a; Glendinning et al., 2000b), from a previous health policy advisor to Tony Blair (Le Grand, 2007) and from Government (Brown, 2008; Milburn, 2007) (Glasby et al., 2009). The Coalition Government reaffirmed the importance of offering personalised services and actively involving individuals, carers and families in all decisions concerning services and support within the NHS in a number of policy documents. The 2010 White Paper Equity and Excellence: Liberating the NHS highlighted the need for patients to be involved in all decision making: "Shared decision-making will become the norm: no decision about me without me" (HM Government, 2010b). Following the White Paper, the Department of Health published two consultations outlining proposals to secure shared decisionmaking over care and treatment. It was proposed that personalised care planning would be the main way to encourage choice and control among patients (Department of Health, 2012; Department of Health, 2010). In 2011, the Government response to the NHS Future Forum report repeated and emphasised the importance of public involvement: " $A$ health system where patients and the public have a stronger voice and more control - no decision about me without me" (HM Government, 2011).

There are a number of principles underlying the personal health budget initiative (Department of Health, 2009), including:

1. Recipients knowing the resource level available within the budget following an initial assessment and the identification of outcomes.

2. Encouraging patients to develop a support/care plan that details how the resource will be used to meet their identified needs. This should include flexibility in the services and support that can be purchased, but contains a number of restrictions on the use of personal health budgets. The following are excluded:

- Part-funding treatment alongside patients' own money;

- Primary medical services (i.e. GP services) and emergency services;

- Anything illegal, gambling, debt repayment, tobacco and alcohol.

3. Patients deciding how they would like the budget to be managed. There are three options (or potentially a combination of them): notionally, where the budget is held by the commissioner, but the budget holder is aware of the treatment/service options and their

\footnotetext{
${ }^{1}$ www.longtermconditions.dh.gov.uk/about/
} 
financial implications; managed by a third party; or as a direct payment (in certain approved sites only).

It is clear from the underlying principles that co-production is central to the personal health budget initiative. Co-production can be traced back to the 1970s, when the term was introduced as a way of improving service efficiency by actively involving people who use services (Needham and Carr, 2009). In support of this view, a number of recent policy documents have provided a strong direction for coproduction within the personalisation agenda, including the 2010 White Paper, Equity and

Excellence: Liberating the NHS that outlined the importance of actively involving individuals, carers and families in all decisions in the delivery of health care services to ensure that personalised support is being offered (HM Government, 2010b).

The premise of co-production can be found within policy documents related to the introduction of three previous initiatives (direct payments; In Control approach and Individual Budgets) within social care in England. All three initiatives focused on placing more choice and control in the hands of service users in terms of how and when their services and support are delivered.

\subsubsection{Direct payments}

Local authorities were permitted to make cash payments to people with a disability in lieu of community care services following the implementation of the Community Care (Direct Payments) Act (1996). In 2003, after the implementation of the Health and Social Care Act 2001, local authorities were required to offer direct payments to all people eligible for social care services, to be mainly spent on personal and domestic support, through the employment of personal assistants (Department of Health, 2003).

\subsubsection{In Control approach}

Following the direct payment initiative, the White Paper Valuing People (Department of Health, 2001) set out the Government's vision for providing new opportunities for people with learning disabilities. Building on the strategy, the social enterprise organisation In Control combined both person-centred and direct payments, with the introduction of the term 'self-directed' support (Needham, 2011). In Control developed a system of seven-stage self-directed support with the aim of replacing the conventional care management arrangements. The approach encouraged greater flexibility, and the use of a wide range of services and support, rather than solely personal care (Duffy, 2005).

\subsubsection{Individual (personal) budgets}

Building on direct payments (DPs) and the In Control approach, individual budgets (IBs) were first proposed within the Cabinet Office Strategy Unit report Improving the Life Chances of Disabled People (Cabinet Office, 2005) that emphasised the importance of reducing fragmentation and multiple assessments by combining funding streams. This proposal went further than previous initiatives concerning DPs, by offering greater transparency over the level of the budget and how it is calculated; achieving greater flexibility over how the resource is used; and avoiding some of the 
responsibilities believed to have had an impact on the take-up of direct payments. Budget holders were given a choice in how the resource was deployed: they could receive the money directly; have a managed budget via the local authority, third party or a service provider; establish a trust fund; or a combination of these options. Furthermore, individual budgets combined a number of funding sources other than social care, including independent living and supporting people funds, access to work, and disabled facilities grants. Since the individual budget pilot programme, personalisation has continued to be stated in recent key policy documents in England, including the White Paper Building the National Care Service (HM Government, 2010a) which anticipated that, by the time the service is introduced, every eligible person will be offered a personal budget. Unlike individual budgets, personal budgets consist of only social care funds given to service users after an assessment to meet their social care needs. Budget holders should be given a transparent allocation of money, and they are given choice on how to manage the resource and what services are purchased.

The importance placed on greater choice and control in service delivery is not focused solely in England, with a number of reviews (The Health Foundation, 2010; Alakeson, 2010a; Alakeson, 2010b; Arksey and Kemp, 2008; Timonen et al., 2006; Ungerson and Yeandle, 2007; Alakeson, 2007; Pavolini and Ranci, 2006) highlighting the development of self-directed support initiatives in various countries, including Austria, Belgium, Denmark, Italy, Finland, Austria, France, Sweden, Germany, Australia and the USA. The research scan carried out by The Health Foundation (2010) outlined that the focus of most programmes was to reduce the cost of health and social care budgets, although it was highlighted that the motivations for introducing personal budgets differed between countries. For example, in Belgium the initiative was designed to reduce the use of residential care, while in Australia the focus was on reducing the fragmentation of services in remote rural areas.

Furthermore, there were differences between the initiatives as to the target population. In the USA, the 'cash and counselling system' is only available for people eligible for Medicaid, while in the Netherlands elderly and disabled people in need of care can apply to a special municipal agency for care services. In contrast, Germany has tested the impact of using personal budgets for people in need of nursing care (The Health Foundation, 2010).

\subsection{Research evidence}

A number of reviews have provided an overview of the social care initiatives outlined above and have found generally a positive impact on people's satisfaction with services and quality of life (Carr, 2011; Carr and Robbins, 2009; Needham and Carr, 2009; Needham, 2011; Glasby and Littlechild, 2009; The Health Foundation, 2010; Alakeson, 2010b; Glasby et al., 2009).

Research focusing on the impact of personalised support within the health care system in England is limited and is often based on qualitative or non-controlled studies (NHS Confederation and National Mental Health Development Unit, 2011; NHS Confederation and National Mental Health Development Unit, 2009; Cole, 2009; Macmillan Cancer Support, 2008). Although the examples of previous research are of great interest and provide valuable evidence, the studies were not designed to answer questions such as whether personalised support can ensure better outcomes when compared to conventional service delivery. From a number of reviews of the existing evidence (Alakeson, 2010a; Alakeson, 2007; Alakeson, 2010b) it has been suggested that the evidence from 
the US (particularly from randomised controlled trials) provides support for the notion that giving patients more choice and control could have a positive impact on patients' experiences of NHS services in England. Alakeson (2007) provides an example of a controlled experimental study that explored the impact of a Cash and Counselling programme in the US on costs and outcomes. This programme offers Medicaid patients more choice about how to get help at home. The initiative was first launched as a demonstration project in Arkansas, Florida and New Jersey. According to the evaluation of the demonstration projects that was carried out between 1998 and 2002, consumers were overwhelmingly satisfied with the Cash and Counselling programme (Robert Wood Johnson Foundation, 2006). The evaluation found that, compared to a control group, Cash and Counselling reduced participants' unmet needs for care and helped them maintain their health. It also significantly improved the lives of carers. The three states found that the programme could be implemented without costing substantially more than traditional services.

\subsubsection{Evaluation of the personal health budget pilot programme}

Following the publication of the NHS Next Stage Review (Department for Health, 2008), the personal health budget pilot programme was launched by the Department of Health in 2009 (Department of Health, 2009). The Department of Health invited PCTs to become pilot sites and to join a three-year programme to explore the opportunities offered by personal health budgets; and an independent evaluation was commissioned to run alongside the pilot programme. The 2010 White Paper Equity and Excellence - Liberating the NHS (HM Government, 2010b) and the Government response to the NHS Future Forum report (Department of Health, 2011) outlined that the Government intended to use the results from the evaluation of the personal health budget pilot programme to inform a wider, more general roll-out of the initiative after 2012.

However, for a national roll-out to be successful, robust, systematic evidence is required. It is essential that the various implementation possibilities for personal health budgets and the impact for different individuals be fully explored; personal health budgets cannot be viewed as a one-size-fits-all policy. The various complexities within the personal health budget process need to be fully understood if the potential of personal health budgets is to be realised after the pilot programme.

As noted above, much of the previous evidence exploring the impact of personalised support on health outcomes and on the system has involved qualitative or non-controlled research. This study took a longitudinal approach combining both quantitative and qualitative data collection points not only to bridge the gap in the evidence but also to be able to provide a complete account of the personal health budget process from the system, and from patients and carers perspectives.

Initially, 64 pilot sites were involved in piloting personal health budgets and contributing to the evaluation. During the evaluation period, the pilot programme remained open; 75 pilot sites were implementing personal health budgets at the time of writing. Most of the pilot sites were based within an individual PCT, although some worked across a number of PCTs.

Twenty sites from across the pilots were selected to be in-depth evaluation sites, with the remainder being wider cohort sites. The 20 in-depth pilot sites each received funding of $£ 100,000$ per year (for 
three years in total) to ensure that the requirements of the evaluation were met. The wider cohort received lower levels of funding per year as the evaluation demands on them were less onerous.

The 20 in-depth pilot sites were selected using the following criteria:

- Pilot sites offering personal health budgets to a large enough group of patients aged 18 and over;

- Pilot sites offering budgets to patient groups where there were enough patients in total for the evaluation to provide useful information;

- Pilot sites offering a mixture of deployment options;

- Pilot sites enabling inclusion of a range of socio-economic groups, age groups, ethnic groups and urban/rural populations; and

- Pilot sites implementing personal health budgets quickly enough to contribute information to the evaluation.

Based on the plans submitted by pilot sites, the in-depth pilot sites offered personal health budgets to individuals with the following health conditions: long-term conditions (including chronic obstructive pulmonary disease, diabetes and long-term neurological conditions); mental health; NHS Continuing Healthcare; and stroke. Table 1-1 below outlines the characteristics of the pilot sites.

Table 1-2 outlines the models of how personal health budgets were implemented among the indepth pilot sites. A range of implementation methods were used for personal health budgets, varying according to: whether the budget is known before support planning; what flexibility there is in terms of what help can be purchased; and the choice of deployment (including direct payment). The range of specification options were condensed into five models by the evaluation team. Model 1 is regarded as the most ambitious being nearest to policy intentions for personal health budgets. Model 5 is a combination of models 1 and 2. The only difference between these models is the existence of a menu of services for the budget holder to choose from, which was as wide as possible to provide flexibility to the budget holder. Nineteen of the 20 in-depth pilot sites were classified within one of the models. Quotations from the qualitative interviews with organisational representatives following each implementation model can be found in Appendix D. 


\begin{tabular}{|c|c|c|c|}
\hline $\begin{array}{l}\text { Pilot } \\
\text { sites }\end{array}$ & $\begin{array}{l}\text { Type of local } \\
\text { authority }\end{array}$ & $\begin{array}{l}\text { Strategic Health } \\
\text { Authority }\end{array}$ & $\begin{array}{l}\text { Health condition initially chosen for the personal } \\
\text { health budget pilot }\end{array}$ \\
\hline 1 & Metropolitan & Yorkshire and Humber & Mental health; NHS Continuing Healthcare \\
\hline 2 & London & London & COPD; Diabetes \\
\hline 3 & Unitary & Yorkshire and Humber & $\begin{array}{l}\text { COPD; Diabetes; Long-term neurological; NHS } \\
\text { Continuing Healthcare; End of Life }\end{array}$ \\
\hline 4 & Unitary & West Midlands & COPD; Diabetes; Mental health \\
\hline 5 & Shire & South Central & NHS Continuing Healthcare \\
\hline 6 & Shire & South East Coast & $\begin{array}{l}\text { Mental health; NHS Continuing Healthcare; End of } \\
\text { life; Maternity }\end{array}$ \\
\hline 7 & Shire & East Midlands & $\begin{array}{l}\text { Long-term neurological; NHS Continuing healthcare; } \\
\text { Stroke }\end{array}$ \\
\hline 8 & London & London & COPD; Diabetes; Stroke \\
\hline 9 & Shire & South West & $\begin{array}{l}\text { COPD; Long-term neurological; NHS Continuing } \\
\text { Healthcare; End of life }\end{array}$ \\
\hline 10 & Unitary & South East Coast & $\begin{array}{l}\text { COPD; Long-term neurological; Mental health; } \\
\text { Stroke }\end{array}$ \\
\hline 11 & Unitary & North East & $\begin{array}{l}\text { COPD; Long-term neurological; NHS Continuing } \\
\text { Healthcare }\end{array}$ \\
\hline 12 & Metropolitan & West Midlands & COPD; Diabetes \\
\hline 13 & Unitary & East Midlands & $\begin{array}{l}\text { Long-term neurological; Mental health; NHS } \\
\text { Continuing Healthcare }\end{array}$ \\
\hline 14 & Shire & East of England & $\begin{array}{l}\text { COPD; Long-term neurological; Mental health; NHS } \\
\text { Continuing Healthcare }\end{array}$ \\
\hline 15 & Unitary & South West & Long-term neurological; Mental health \\
\hline 16 & Unitary & South West & Stroke; NHS Continuing Healthcare \\
\hline 17 & Metropolitan & North West & Mental health \\
\hline 18 & Metropolitan & North West & NHS Continuing Healthcare \\
\hline 19 & Unitary & East of England & Long-term neurological; Stroke \\
\hline 20 & London & London & $\begin{array}{l}\text { COPD; Diabetes; Long-term neurological; NHS } \\
\text { Continuing Healthcare; Stroke }\end{array}$ \\
\hline
\end{tabular}


Personalised budget is known before support planning

Flexibility in what help can be purchased

Deployment choice (including DP)

Model 2

4 pilot sites

Budget is known before support planning (but may not be

personalised - a set amount)

Service directory

Deployment choice (including DP)

Model 3

3 pilot sites

Budget is known before support planning (but may not be

personalised - a set amount)

Lack of flexibility in the help that can be purchased

No deployment choice

Model 4

4 pilot sites

Budget is not known before support planning

Flexibility in what help can be purchased

Variation in the degree of deployment choice

Model 5

Model 1 and 2 combined

\subsubsection{Aims of the national evaluation}

The overarching aim of the evaluation is to identify whether personal health budgets ensured better health and social care outcomes when compared to conventional service delivery and, if so, the best way they should be implemented. The evaluation explores:

1. The process of implementing personal health budgets for individuals and carers;

2. The short- and longer-term impacts of personal health budgets on different groups of people and their carers (including the effects of demographic and socio-demographic factors);

3. The cost-effectiveness of implementing personal health budgets for different health conditions, compared to conventional service delivery; and the costs and benefits of different models of personal health budgets for different groups of people;

4. The short- and longer-term impact of implementing personal health budgets on NHS organisations, staff and the wider health and social care system;

5. The fit of personal health budgets with the broader context of the NHS and NHS values; and

6. The longer-term effects if personal health budgets were to be further rolled out. 


\subsection{Structure of the report}

This report is structured as follows. Chapter 2 outlines the methods used in the evaluation. Chapter 3 describes the sample of participants recruited to the study. Chapter 4 provides a description of how people were using their personal health budget. Chapter 5 reports the analysis of the impact of personal health budgets on a range of outcome indicators. Costs and cost-effectiveness analysis are reported in chapter 6 , while chapter 7 describes the analysis of the impact of receiving personal health budgets on carers. Chapter 8 discusses the main analysis to infer the successes and challenges of the personal health budget pilot. Finally, chapter 9 draws together the evidence to provide a series of recommendations about whether, and how, personal health budgets should be implemented beyond the pilot. 


\section{Methods}

\subsection{Summary}

The evaluation used a controlled trial with a pragmatic design (depending on pilot site arrangements) to compare the experiences of people selected to receive personal health budgets with the experiences of people continuing under the current support arrangements for their condition.

The quantitative and qualitative data collection points are outlined in this chapter that were followed to explore:

- Patient outcome and experiences

- Individual outcome data;

- Primary and secondary care service use;

- Information about care/support plans;

- Qualitative data on patient outcomes and experiences;

- Implementation experiences and costs

○ Pilot sites' experiences of implementing personal health budget.

The chapter introduces the main data analyses and outlines the challenges in implementing the evaluation design.

\subsection{Overall research design}

The evaluation within the in-depth pilot sites used a controlled trial with a pragmatic design (depending on pilot site arrangements) to compare the experiences of people selected to receive personal health budgets with the experiences of people continuing under the current support arrangements for their condition.

To best fit with the specific local configuration of the personal health budget pilot in each site, selection of individuals into either the personal health budget group or the control group occurred in one of two ways. The first option applied to sites where the pilot was set up so that personal health budgets were potentially offered by any health professional in the site. In this case, people under the care of the health professional who were judged as potentially eligible for a personal health budget were randomised into either the personal health budget group or the control group. People selected to the personal health budget group were offered a budget and were asked to participate in the evaluation. The offer of the personal health budget was not dependent on participating in the research. People selected into the control group were also asked to participate in the evaluation.

The second option was where pilots were set up so that separate health professional groups either offered personal health budgets or recruited patients to the control group. This meant that all potential personal health budget patients, up to a quota, were selected into the PHB group by participating health professionals. Non-participating health professionals in that site were asked to select a control group from people who would potentially be eligible for a personal health budget. A cluster randomisation design was considered, with GP practices forming clusters of patients. 
However, although the number of GP practices across the 20 in-depth pilot sites was sufficiently large, the numbers within each pilot site were not. Given the different implementations of personal health budgets by site, selection needed to occur within each pilot site. Instead, comparator practices were chosen according to local selection criteria. In the main, personal health budget patients were drawn from practices in one patch within the pilot site and the control patients from practices in a different patch (chosen to be a similar as possible). Where this was not possible, for example because local GPs refused to offer personal health budgets or where equivalent patches could not be easily found, control patients were drawn from practices selected on a more opportunistic basis.

For people who did not have the capacity to consent, (for example where an individual lacks capacity to make a decision or take a particular action for themselves at the time the decision needs to be taken), a representative was approached and asked if they would like to participate in the evaluation on behalf of the patient. The representative was asked to give consent for the potential participant to take part only if they believed this would be their wish. The decision about capacity to consent was initially made by the identified health professional in the pilot site.

The allocation into either the personal health budget group or the control group was followed by a number of quantitative and qualitative data collection points over a 12-month period.

\subsection{Data collection for in-depth sites}

Figure 2-1 outlines both quantitative and qualitative data collection that was followed to explore both:

- Patient outcome and experiences;

- Implementation experiences and costs.

Figure 2-1 provides further detail on the different data collection points and the outcome measures.

\subsubsection{Patient outcome and experiences}

\subsubsection{Individual outcome data}

Within the quantitative data collection, outcome data were collected on four occasions: at the time of consent (baseline); six months after date of consent (initial follow-up); 12 months after date of consent (main follow-up); and up to 24 months after date of consent (second follow-up).

Organisational representatives working within the pilot sites carried out the baseline outcome interviews between April 2010 and June 2011.

The six-month data collection was via postal questionnaires which were sent out from October 2010 and continued until December 2011. The follow-up interviews began 12 months later, in April 2011 and continued until June 2012; these interviews were conducted by a research fieldwork agency. Finally, the second follow-up outcome data collection was via a postal questionnaire which was sent out between 17 and 24 months later, starting in September 2011 and continuing until May 2012. 
The questionnaires included the following outcome measures:

- Health-related quality of life (EQ-5D - Euro-QoL);

- Care-related quality of life (ASCOT);

- Psychological well-being (GHQ-12);

- Subjective well-being scale;

- Perceived quality of life (a seven-point scale);

- Perceived health (a five-point scale).

Further details can be found below (section 2.5.5) and in Appendix A. The outcome questionnaire also collected information around social care and primary care service use. In addition, demographic and socio-economic information was collected, as well as information about current circumstances. Table 2-1 lists the variables that were included in the outcome questionnaires.

Table 2-1 List of variables included in the outcome questionnaire

\begin{tabular}{ll}
\hline Demographic information & Socio-demographic information \\
\hline Age & Highest education level \\
\hline Ethnicity & Type of income \\
\hline Sexual orientation & \\
\hline Gender (including trans-gender) & Current circumstances \\
Religion & Activities of daily living \\
\hline Household composition and accommodation & Receipt of informal care support \\
\hline Marital status & Employment status \\
\hline
\end{tabular}

\footnotetext{
${ }^{2}$ (c) 1990 EuroQol Group. EQ-5D ${ }^{\mathrm{TM}}$ is a trade mark of the EuroQol Group.
} 


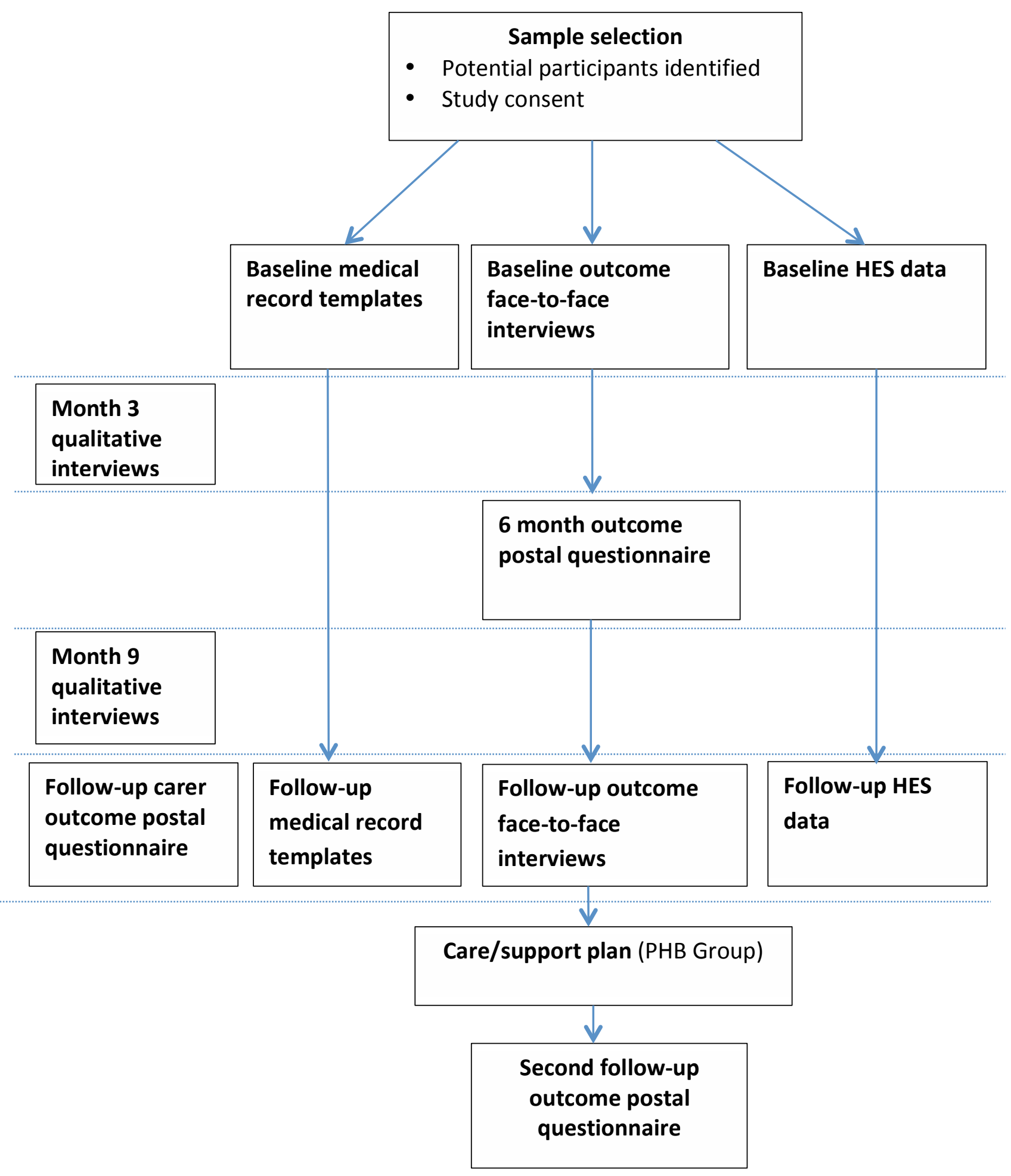




\subsubsection{Primary and secondary care service use}

A medical record template was designed by the evaluation team to gather information from GP records concerning participants' health status and their use of primary and secondary health care services.

This information was collected at two-time points during the study period: first, around the time of consent to explore the previous 12 months activity; second, around 12 months after participants agreed to take part to gather information for the year following consent. Information about secondary health care service use was also gathered from Hospital Episodes Statistics (HES) during 2008 and 2012.

\subsubsection{Information about the care plan and personal health budget}

For the personal health budget holders, the evaluation team asked for a copy of the support/care plan that outlined the following information:

- The budget per year, and the total level of funding in terms of health service expenditure, recurrent annual and one-off payments (where applicable);

- The cost of planning health support;

- The formal organisation of the budget in terms of deployment options;

- The activities in the care plan that the budget was to be spent on; and

- The cost of the individual services identified within the care plan.

\subsubsection{Qualitative data on patient outcomes and experiences}

The quantitative arm of the study was supplemented with qualitative data derived from semistructured interviews with a sub-sample of personal health budget holders. These interviews were conducted three and nine months after the offer of a personal health budget. Budget holders were recruited for these interviews from amongst those who had already consented to take part in the quantitative strand of the evaluation. The aim was to recruit this sub-sample from at least half of the 20 pilot sites involved in the in-depth evaluation; to include budget holders with each of the health conditions for which personal health budgets were offered; and to include a spread of ages and a roughly equal gender balance. Sampling also aimed to include budget holders from a range of ethnic groups. However, this depended on the wider sample and, as only small numbers of non-White British budget holders had been recruited to the wider evaluation at the time the sub-sample was selected, the number of interviewees from minority ethnic communities in the sub-sample was similarly limited. Inclusion of the full range of deployment options for personal health budgets in the qualitative sub-sample was similarly determined by progress with implementation in the pilot sites and by recruitment to the wider evaluation.

The aim was to recruit a sub-sample of 55 budget holders to take part in the semi-structured interviews. In fact, 58 budget holders were recruited initially and took part in the three month interviews; 52 of these were re-interviewed at nine months. Five of the initial sample withdrew 
before the nine-month interview, and a sixth who had been interviewed at three months could not be re-contacted at nine months.

The interviews at three months explored how people found out about personal health budgets and their initial impressions; their knowledge of the amount of the budget and how this had been calculated; their decisions about how to use the budget and the help received with this; and options for managing the budget. The nine-month interviews focused on the perceived impacts of the personal health budget on budget holders' health, well-being and quality of life; reflections on their chosen uses for the budget; satisfaction with the amount of the budget; reflections on the way the budget had been managed; and experiences of the implementation of personal health budgets. Interviews were digitally recorded, with interviewees' consent, and subsequently transcribed.

\subsubsection{Carers}

Personal health budgets may have an impact not only on patients but also on their family carers. We asked study participants to say whether they had a carer and, if so, whether they were happy for us to approach them. Twelve months after the patient gave consent, a questionnaire was made available to those carers in both the personal health budget group and the control group. This data collection began in April 2011 and continued until June 2012. The questionnaire included the following outcome measures to explore whether personal health budgets had an impact on carers' quality of life and the caring role:

- Health-related quality of life (EQ-5D - Euro-QoL);

- Perceived quality of life (a seven-point scale);

- Perceived health (a five-point scale).

The questionnaire also contained questions from the first national survey of adult carers' experiences, views and outcomes conducted in England by councils with social services responsibilities (Malley et al., 2010; Fox et al., 2010; Holder et al., 2009).

\subsubsection{Implementation experiences and costs}

\subsubsection{Pilot sites' experiences of implementing personal health budgets}

Both quantitative and qualitative data collection techniques were used to explore pilot sites' experiences of implementing personal health budgets.

The quantitative data collection involved key front-line operational staff completing a web-based questionnaire in month 3 and month 24 of the evaluation. The questionnaires focused on whether the implementation of personal health budgets had an impact on the workplace environment. The quantitative data also included various relevant measures, such as 'propensity to leave', job satisfaction and occupational stress.

In addition, to ensure as far as possible that the costs reported reflected only the resources required for the implementation of personal health budgets rather than being associated with being a pilot site, a template was designed (see Appendix E). The data collection explored the costs associated 
with implementing personal health budgets during the first and second year of the local pilot programme. Project leads were asked to complete the template at two time-points: during month 12 and month 24 of the evaluation.

It was anticipated that the implementation of personal health budgets would create many challenges for the pilot areas. It was vitally important to explore in detail the processes of implementing personal health budgets; the challenges and barriers encountered and how these were overcome; and the impact of personal health budgets on the wider local health and social care economy and patterns of service provision. This area of the evaluation relied on qualitative interviews from project leads; operational staff; front-line professionals; third-party budget holders; and commissioning managers at three points of the study period: months 3, 15 and 24. Interviews with service providers and managers of user/carer organisations were carried out within month 24 of the evaluation.

\subsection{Data collection for the wider cohort pilot sites}

The wider cohort of pilot sites was asked to recruit personal health budget holders to the main evaluation between April 2010 and April 2011. Recruitment to the personal health budget group followed the process outlined for the in-depth pilot sites.

Upon receipt of the consent form, the baseline outcome questionnaire was posted directly to the individual for completion. An outcome questionnaire was sent again at 12 months after the person consented to participate in the evaluation. The questionnaire covered the same measures as described above. As with the data collection in the in-depth sites, a copy of the care plan was requested, and information about the budget holder's use of secondary care services was extracted from the hospital episode statistics (HES).

To provide further understanding of the implementation issues within the local wider cohort pilot, a web-based questionnaire was developed for project leads to complete during month 17 of the study period. A further web-based questionnaire was developed to capture the views of project leads around the perceived success of the local pilot.

\subsection{Quantitative data analysis}

\subsubsection{Sample size and power}

Based on initial power calculations, the aim was to achieve a final sample size of 2,000 individuals into the evaluation: 1,000 to the personal health budget group and 1,000 to the control group. Power calculations for minimum sample sizes were computed using data from the evaluation of individual budget pilot programme (IBSEN) (Glendinning et al., 2008). In common with the measures used for this study, the IBSEN evaluation used both the GHQ-12 measure and the ASCOT measure ${ }^{3}$.

\footnotetext{
${ }^{3}$ A slightly different version of ASCOT was available at the time.
} 
Based on the mean and standard deviation scores for the comparison sample in the IBSEN study, and assuming a power of 0.8 , a minimum sample of 1,474 (737 participants recruited to the 'personal health budget group' and 737 to the 'control group') would be required to detect an overall onepoint increase in GHQ-12 in a two-sample comparison. ${ }^{4}$

As regards ASCOT, the IBSEN study detected an improvement of just over 0.03 (significant at the $5 \%$ level) when expressed on a 0 to 1 range. To detect this change in a two-sample comparison based on the IBSEN comparison group mean and standard deviation would require each sample to have at least 635 observations or 1,270 in total.

Given that we expected some loss at follow-up and also missing data within records that would require imputation (see section 2.5.4 below), we aimed to achieve a sample size of 1,000 for each group (personal health budget and control) by having an initial target sample of 3,000 participants to account for attrition rates. Ideally a larger sample would have been collected to allow sub-group analyses to be powered, but there were limits on the resources available in the study. As a consequence, for sub-group analyses with fewer cases than indicated by our power calculation a non-significant result could stem from the estimation being under-powered.

\subsubsection{Evaluating impact within the in-depth pilot sites}

To assess the impact of any intervention - such as a personal health budget or a new drug - on an outcome indicator we need to establish the counterfactual: i.e. what would have happened if the person had not received the intervention. The difference between the indicator with the intervention and the indicator without the intervention is a measure of its effectiveness. One way to do this is a before-and-after study: that is, to assume that the person's situation/status before the intervention is a good indication of what their situation would be in the future had they not had the intervention. However, this is a strong assumption: that over the course of the intervention nothing else affects people's situation. This assumption is particularly hard to defend when the intervention takes place over a long period of time, e.g. a year, and where it involves people with one or more long-term health conditions which could deteriorate during the study period.

An alternative is to use a control group and to compare the experiences of people who had the new intervention with those who continued to receive their usual support. A comparison-after-use study measures the outcome indicator of interest in both groups after the intervention and takes the difference as a measure of effectiveness of the new intervention. This approach assumes that the situation of people before the intervention is the same between the personal health budget and control groups. In a randomised controlled trial we can be confident that this is the case, especially if the trial is 'blind': i.e. that patients and practitioners do not know which group they are in during the study.

\footnotetext{
${ }^{4}$ The 36-range GHQ-12 score in IBSEN had a mean of 13.8 and a standard deviation of 6.85 for the comparison group.
} 
In many cases a double-blind randomised controlled trial is not possible, and it was not possible in this evaluation. Without blinding there was the potential problem that local patients and practitioners could change their behaviour with regard to the control group, thus potentially biasing the experiences of participants in providing a counterfactual. This problem is particularly significant for evaluation of more systemic interventions such as personal health budgets rather than, say, a new drug therapy.

In this study we chose a mixed methodology, with some in-depth sites using (non-blind) randomisation and others using a control between localities, as described above. This mixing gave us some opportunity to compare results produced by these different methods. In any case, it was difficult to defend the assumption that the characteristics and situation of people in the personal health budget group were the same (on average) as those people in the control group. To address this potential limitation, we opted to use a method that required the weakest assumptions: the difference-in-difference approach. This approach recognises that both intervention and control groups could differ at baseline and that other influences may apply during the course of the intervention. Rather than compare post-intervention outcome indicators between groups, we compared the changes in the outcome indicator between baseline and follow-up between the two groups. In particular, effectiveness was measured by subtracting the change (follow-up score minus the baseline) in the outcome indictor for the control group from the same change in the outcome indicator for the intervention group. In other words, we were assuming that, without the intervention, the situation of the intervention group would change (on average) by the same amount as the control group. The counterfactual is therefore the change in outcome indicator between the groups. Arguably, this assumption - that external influences affect people in both groups in the same way - is less demanding than the assumptions required for the above methods.

The difference-in-difference (DiD) measure was:

$\Delta y=\left(y_{11}-y_{10}\right)-\left(y_{01}-y_{00}\right)$

where:

$y_{11}$ is the measured indicator for the personal health budget group at follow-up

$y_{10}$ is the measured indicator for the personal health budget group at baseline

$y_{01}$ is the measured indicator for the control group at follow-up

$y_{00}$ is the measured indicator for the control group at baseline

The method for deriving this difference-in-difference measure is described in Box 2-1. Further information can be found in Appendix $C$. 


\section{Box 2-1. Difference-in-Difference methods}

Suppose that $y_{i t}$ is the outcome indicator in question (e.g. care-related quality of life (SCRQOL). The subscript $i$ refers to the patient group: $i=1$ for the personal health budget group and $i=0$ for the control group. The subscript $t$ refers to the time point: $t=0$ is the pre-intervention baseline time and $t=1$ is the post-intervention follow-up time (e.g. the 12month follow-up). In the figure below () we have illustrated a situation where the personal health budget group has higher (better) SCRQOL than the control group i.e. $y_{10}>y_{00}$, perhaps because slightly healthier people were selected into the PHB group. In fact, the difference in SCRQOL is $y_{10}-y_{00}=\beta_{1}$. By the follow-up time, the measured difference between groups is $y_{11}-y_{01}$, but on its own this difference would over-estimate the impact of personal health budgets because it does not account for the difference in SCRQOL at baseline. If we assume that the personal health budget group of people would have deteriorated in terms of their SCRQOL at the same rate as the control group, i.e. by an amount $\beta_{2}$, then without the intervention they would have averaged SCRQOL of $\hat{y}_{11}$, not $y_{01}$, and therefore the 'true' improvement is the amount $\beta_{3}$. Another way of thinking about this approach is to compare measured SCRQOL for the personal health budget group between baseline $\left(y_{10}\right)$ and follow-up $\left(y_{11}\right)$. This difference would under-estimate the effect of the intervention in this example because without the intervention, and taking a lead from the control group, we would have expected SCRQOL to have fallen by $\beta_{2}$ to a level $\hat{y}_{11}$. So again, the 'true' effectiveness is $y_{11}-\hat{y}_{11}=\beta_{3}$. As can been seen, this is equivalent to the calculation $\Delta y=\left(y_{11}-y_{10}\right)-\left(y_{01}-y_{00}\right)$ as set out in the text.

\section{Figure 2-2. Difference-in-Difference methods}

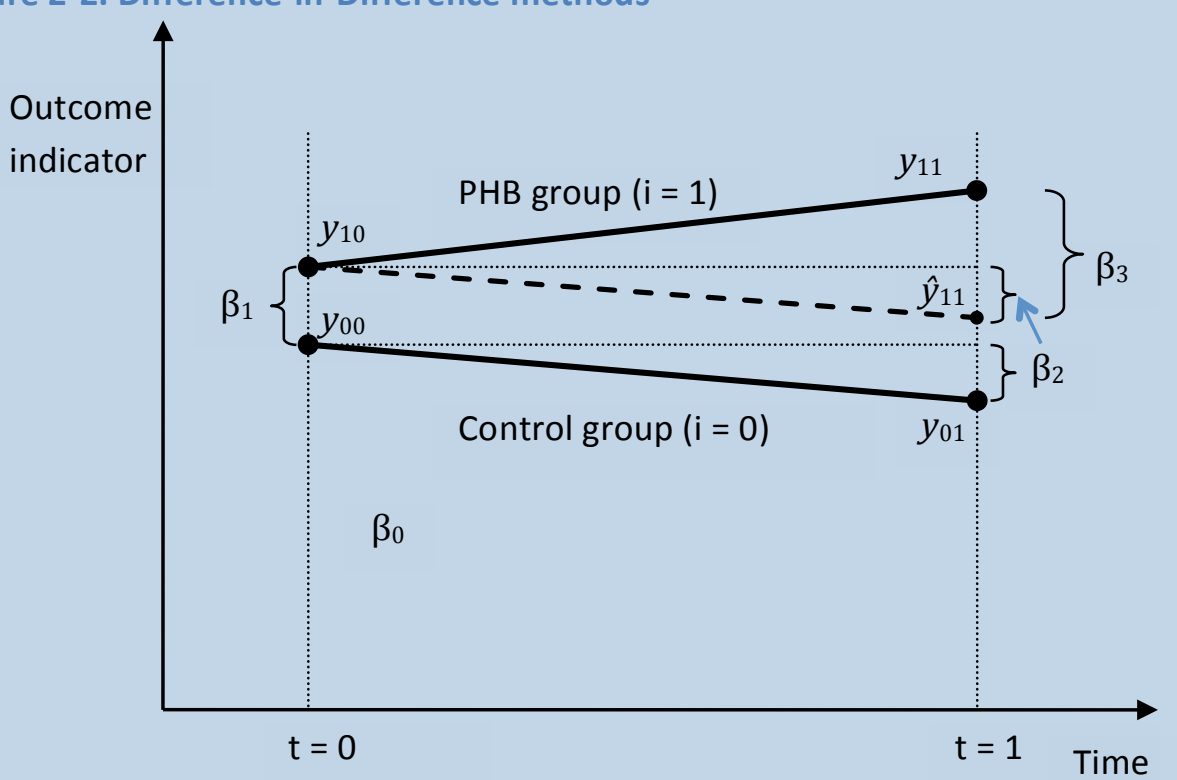

The difference-in-difference method can be used for any indicator that has been measured both at baseline and follow-up. In particular, we used it for our effectiveness measures, where we have $y$ as an effect indicator, $E$, such as health or care-related quality of life. The difference-in-difference 
estimate is then change in effect $\Delta E$. Also, the same method could be used for the cost of the care, services and support a person receives, so therefore we measured change in cost: $\Delta C$.

\subsubsection{Sub-groups}

The evaluation concerned a number of patient groups as potential beneficiaries of personal health budgets as outlined in Chapters 1 and 3. In each case we had the option to separate out these subsamples completely and run the analysis for these groups on a stand-alone basis. This approach clearly meant smaller sample sizes for the sub-samples. Moreover, it did not account for any similarity of effect (of personal health budgets) that applied for each sub-sample. The alternative was to analyse the whole sample but with dummy variables to distinguish patient groups. These dummy variables could be used to account for any differences at baseline (intercept dummies) and also differences in effect through time (interaction dummies).

\subsubsection{Confounding factors on outcome indicator changes}

In using a difference-in-difference approach we effectively remove any differences between the groups in the level of the outcome indicator at baseline. Whilst this method is an effective way to control for any differences in the characteristics of the personal health budget and control groups affecting the outcome indicator at baseline, there remains a possibility that differences between the groups might cause differences in the rate of change of the indicator, beyond any true effect of personal health budgets - see Appendix C. To safeguard against the possibility of bias of this kind, we also estimated multivariate difference-in-difference models. These allow us to identify and remove the effects of differences in baseline characteristics between the groups on change in the outcome measure. A number of baseline characteristics were explored in these 'controlled' analyses, including socio-demographic factors (for example, gender, age, baseline dependency, accommodation, ethnicity); socio-economic factors (for example, education, benefit receipt); and health status (for example, health condition and comorbidities) (see Appendix $C$ for the full list of confounding variables).

\subsubsection{Mortality}

Mortality rates are an important outcome measure and we investigated whether mortality was higher in the personal health budget group compared to the control group. In theory, a difference-indifference method can be applied, but in this case there is no difference in mortality at baseline between the groups. A simple comparison of mortality rates is unlikely to be sufficient, however, for the reasons outlined above. Indeed, the chance that differences in baseline characteristics will introduce spurious change bias is particularly important for mortality rate analysis. The lack of difference at baseline can mask significant differences in the probability of death immediately after baseline.

For these reasons, we estimated the probability of dying, controlling for a range of baseline characteristics, particularly morbidity indicators and also study timings. As regards the latter, and discussed in more detail below, for practical reasons the time between consent and baseline and between baseline and follow-up was not always the same for each study participant. Elapsed time is 
highly relevant for analysis of mortality, so for this analysis we selected a sample where time frames were comparable.

\subsubsection{Missing values and loss to follow-up}

In highly complex evaluations, such as PHBE, we will always have at least some missing data for individuals in the study. In some cases, these missing data are where respondents overlook or fail to answer particular questions but manage to complete many others. Where these responses could be considered to be missing at random (to a reasonable extent), we were able to 'impute' values for missing data with techniques that use the underlying patterns in the whole dataset. The Technical Annex (Appendix $\mathrm{C}$ ) has more details of these multiple imputation techniques.

After the baseline period, some original participants withdrew from the study or were unable to continue to participate, or died. The number of people in the study at follow-up (e.g. 12 months) was lower than at baseline. We were unable for ethical reasons to ask people why they had withdrawn if they had rescinded consent. We could assume in some cases that this withdrawal was at random and therefore use multiple imputations to infer characteristics at follow-up.

In Chapter 3 we describe the sample and the numbers of records used in the main analyses. Our overall approach was to include all participants in the analysis where we had either baseline outcome information or any form of outcome follow-up data. A number of study participants were in residential care at baseline. Since residential care is not included in the personal health budget pilot programme, we excluded these cases. With this definition of the active sample, multiple imputation was used to address missing values on all major variables in the dataset.

Following imputation, we excluded anyone below the age of 18. For the main outcomes, cost and cost-effectiveness analyses we also excluded people that had died at follow-up. Although in theory we might assign them outcome indicator and costs of zero at that point, this would not be a good guide to their experience between baseline and follow-up. We instead conducted a separate analysis of death rates.

\subsubsection{Variable follow-up periods}

Due to the different timing of local implementation between sites, delays in recruitment and workload at follow-up, there was some variation in the follow-up periods for participants in relation to our 12-month target duration. The follow-up data collection period in the study was between 9 and 18 months. Without prior knowledge about the timing of the actual impact of personal health budgets on people's outcomes, we could not specifically adjust for these differences, although we did include the time elapsed between baseline and follow-up as a variable in the statistical models.

On average, follow-up periods were similar between the personal health budget and control groups. It might nonetheless be the case that where follow-up was less than 12 months, this gave less opportunity for personal health budgets to show differential outcomes for patients (if such differences existed). We flag this issue as a possible limitation of the evaluation. 


\subsubsection{Outcome indicators}

We used a range of validated measures of impact or effectiveness as outlined above and in Appendix A. The intention was to measure impact in the following categories:

- Clinical outcomes: The aim was to assess the impact of personal health budgets on the health of study participants in terms of standard clinical markers. To this end, we measured $\mathrm{HbA1C}$ for diabetes sufferers and lung function (forced expiratory volume, FEV1) for COPD sufferers. Following advice from a medical professional, there wasn't an appropriate clinical outcome measure for the other health conditions.

- Health-related quality of life: We used the EQ-5D measure, which aims to measure a person's quality of life in domains that are likely to be related to their underlying health status. This measure is well established in research and offers significant potential for comparison of the results with those of other studies. EQ-5D can be summarised as measuring personal functioning (as potentially constrained by poor health) such as mobility, being free of pain and depression, and in order to achieve everyday activities (e.g. self-care, usual activities and mobility).

- Care-related quality of life: We also used another care-related quality of life measure with a focus on the achievement of everyday activities that might come from the support of services and interventions, as well as from personal functioning. Arguably, quality of life improvements for people with long-term conditions come mainly from a management of those conditions rather than any prospect that the underlying condition can be cured. There are a number of indicators that might be appropriate but we wanted a measure that was utility weighted in order to undertake comparative cost-effectiveness analyses. For these reasons we used the ASCOT measure (Adult Social Care Outcomes Toolkit) which aims to measure people's achievement of everyday activities, including basic capabilities such as dressing and feeding, as well as more complex capabilities such as feeling safe, being occupied and having a sense of control. ASCOT recognises that achievement of these activities may come from the support of services and interventions, as well as personal functioning. Whilst it was developed for people using long-term social care, many of the indicators are highly relevant for people with long-term conditions in general. For example, it would be expected that having a sense of control over daily living would be equally important for patients with a long-term health condition as well as for services users with social care needs.

- Subjective well-being: We used a subjective global measure based on the measure used by ONS in the Integrated Household Survey (IHS). This measure used in this study seeks to capture general life happiness and satisfaction. We used a scale that considers satisfaction with life, happiness and satisfaction/worry about the person's health.

- Perceived quality of life: The seven-point scale with categories ranging from 'So good, it could not be better' to 'So bad, it could not be worse'.

- Perceived health: The five-point scale asks respondents to rate their health in general according to five categories ranging from 'Very good' to 'Very bad'. 
- Mortality: As well as the above morbidity and quality of life measures, we also assessed whether there were differences in mortality rates between the personal health budget and control groups.

The range of measures used was intended to capture the broader ramifications of use of personal health budgets. As a process intervention, we expected that personal health budgets would have a direct impact on quality of life and rather more indirect impact (through people's choice of service and support) on clinical aspects of their life.

The measures used take the form of numerical scales or dummy variables. They were also measured at multiple time points in the study. The exception is mortality, where there was no difference between personal health budget and control groups in this outcome (all people were alive), and therefore change in mortality is the same as mortality at follow-up. For this reason we had to use mortality-related characteristics at baseline to control for any differences between the groups prior to the study, rather than the value of the respective outcome (on the above scales) at baseline.

\subsubsection{Costs}

Personal health budgets give recipients the opportunity to buy services and support that would not be ordinarily available, or to use services and support to a different intensity than would otherwise be provided.

Table 2-2 lists a range of services and other forms of support (column 1). Some of these are ordinarily available without a personal health budget and some are not (column 2). Depending on their local configuration, personal health budgets can be used to purchase a sub-set of these services (column 3). In some cases, services might be funded from the personal health budget or provided conventionally. Moreover, a person may have some of their services conventionally funded and others secured from their personal health budget. In other cases, support might only be available using a personal health budget - e.g. 'well-being' services such as art classes or holidays - or only conventionally-funded - e.g. secondary care.

In column 4 we distinguish direct, indirect and neutral services. Direct services are those where a personal health budget holder can use their budget to buy the service and so directly determine the amount of that service they use. Indirect services are those which cannot by secured using a budget but where it has been assumed that the use of these services might be affected as a result of a person having a personal health budget and the direct service choices that person makes with their budget. These could be preventative effects: for example, the use of particular well-being services secured using a personal health budget might reduce people's need for primary care (GP visits). Alternatively, a change in the use of direct services might increase the need for indirect services. Finally, we have neutral services where we expect a personal health budget to have very little or no 
effect on their use. For example, we would not expect the use of a personal health budget to affect the medicines a person might need, at least in the short term. ${ }^{5}$

Table 2-2. Services and support - by availability and personal health budget coverage

\begin{tabular}{lccc}
\hline Service or support type & $\begin{array}{c}\text { Usually } \\
\text { available? } \\
\text { (non-budget) }\end{array}$ & $\begin{array}{c}\text { Covered by } \\
\text { PHB? }\end{array}$ & Affected by PHB? \\
\hline Secondary care & Yes & No & Indirect \\
Primary care & Yes & No & Indirect \\
Drugs & Yes & No & Neutral (excluded) \\
Social care (residential) & Yes & No & Neutral (excluded) \\
Specialist nursing and other health & Yes & Yes & Direct \& Indirect \\
Community nursing and AHPs* & Yes & Yes & Direct \& Indirect \\
Social care (community)* & Yes & Yes & Direct \& Indirect \\
Respite care & Yes & Yes & Direct \& Indirect \\
Equipment & Yes & Yes & Direct \& Indirect \\
Well-being support & No & Yes & Direct \\
Informal care (payment) & No & Yes & Direct \\
\hline
\end{tabular}

* Includes personal assistants

The total cost of the services and support that people might use is calculated by applying a unit cost to the intensity measure of each service. In practice, measuring the use of any service across each of the above 11 categories is demanding for study participants. We simplified this requirement by using existing service datasets where possible, such as the hospital episodes statistics (HES) database and by using a costing methodology that places the lowest demand on respondents (but does require us to make a number of assumptions).

With regard to costing, we identified two ways in which personal health budgets were implemented by sites (see also Chapter 4):

- first, where the personal health budget funded services in a given category in addition to what was usually provided;

- second, where the personal health budget substituted for services in a given service category.

\footnotetext{
${ }^{5}$ Potentially, we have assumed that having a personal health budget might lead to improved outcomes that reduce the need for some drugs (e.g. anti-depressants) and have an impact on pain management, although we expect the effect will be small in magnitude. As a result, we do not measure the impact on drug use and assume this is not different between the personal health budget group and control group. However we do collect information on prescriptions and therefore potentially additional analysis could be carried out in the future.
} 
It was possible for a personal health budget to mix these two options for different categories of service: e.g. the personal health budget substituted for social care services but was used to augment provision of physiotherapy.

These distinctions were used in our comparison of costs for people in the personal health budget group and those in the control group. Our approach to costing was to include all health and social care service costs as listed in the above table. The exception was to exclude services for which we strongly anticipated a neutral effect, e.g. for drug medications.

As suggested by their description, in our costing method the value of in-addition services covered in the personal health budget were added to the cost of conventional services in that category to calculate the total cost in each category. For substitute services, their value in the personal health budget was used as the total cost of services in that service category. The overall total cost for each person at any given time was the summation of the sub-totals for each service category.

On this basis, the costs for a personal health budget holder are as follows:

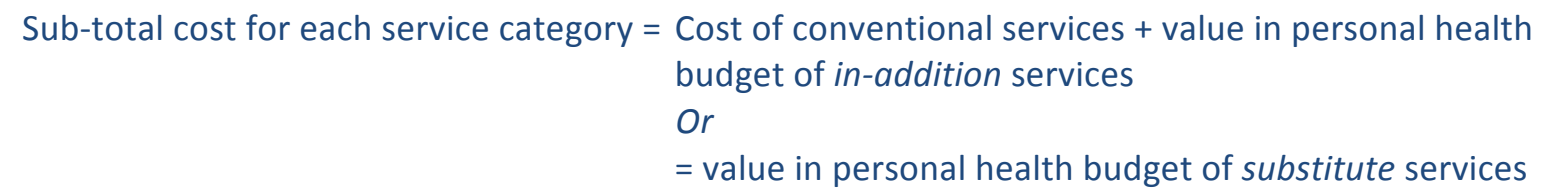

Total cost for personal health budget holder (at follow-up) = sum of sub-total cost for each service

$$
\text { category }
$$

Where all of a person's direct services are covered by the budget, then the total cost is equal to the value of the personal health budget (plus the cost of indirect services). Where budgets are purely additional, then the total cost is equal to the value of the personal health budget plus the cost of the conventionally-funded direct services they receive (plus the cost of indirect services).

In practice, there were a number of data limitations that needed to be addressed. First, we had to identify which services were in addition and which substitute for each service category based on the personal health budget value. In the main analysis we assumed a threshold of $£ 1000$ per annum: that is, personal health budgets of $£ 1000$ or less in each service category were provided in addition to conventional services in that category. Budgets over $£ 1000$ for each category of service were provided as a substitute for conventional service in that category.

Second, although we had direct measures of the cost of services covered by the personal health budget (from the personal health budget care plan), we had a mix of data sources and observations of service intensity for costing the level of conventional services. In particular, for the direct and indirect service categories - i.e. social care, respite, community nursing and AHPs/therapy service categories - we collected data on total use of conventional services in these categories. The difficulty was that we could not be sure whether respondents had included any amount of these services that was personal health budget-funded in addition to that funded in the usual way. This difficulty only 
affects in-addition services where usual and personal health budget funding can be used in the same service category.

Third, we could not collect service-use data on all conceivable, conventionally-funded health and social care services used by study participants. Since all types of service use that are funded by the personal health budget are recorded in the support plan, this created a problem when making likewith-like comparisons between costing people using a personal health budget and those using conventional funding (this includes people in the control group and those people in the personal health budget group at baseline before they received their budget). An example was where nonpersonal health budget holders reported having a social care personal budget but where we did not know its value.

These issues were tackled by making certain assumptions based on our understanding of how sites had implemented the funding and allocation of personal health budgets. As regards the first issue, in most sites budgets provided over-and-above conventionally-funded services were valued at less than f1000 per annum. On this basis we assumed that services costed in the personal health budget care plan at a value of less than $\mathrm{f} 1000$ were in-addition services. Accordingly, services funded in the personal health budget in an amount greater than $£ 1000$ were regarded as substitutes.

Regarding the second issue, we proceeded by estimating the proportion of the total reported use of conventional services that was covered by the personal health budget. This estimation involved comparing the reported total levels of use of substitute services against the amount costed in the care plan. As these are substitutes, there should not have been any non-personal health budget funding. Therefore, the amount of these services reported as the total use gives us an indication across the sample of the proportion of people that were including personal health budget-funded services in the total against those who were not. This sample average proportion was used to reduce the value of total use of conventional services when it was added to the value of personal health budget-funded services in each category to calculate total cost. For example, take the community social care service category. If $25 \%$ of the sample with budgets of over $£ 1000$ for that category had included personal health budget-funded amounts in their reporting of the total use of (conventional) services, then we would use this $25 \%$ figure as a deflator when costing in-addition community social care (i.e. for those with a personal health budget cost of less than $f 1000$ on community social care). Full details of this method are provided in Appendix C.

We had two options for tackling the third data limitation. We could remove the cost of these services covered by the personal health budget from the total. Alternatively, we could predict the amount of these services that would have been used by non-personal health budget holders and use this cost in the total. We chose the latter option because it allowed us to gauge any change in costs in this category over time (where the main analysis is in change in total cost). The predicted value was estimated using the correlation between the amount of these services in the personal health budget and some predictor variables such as health condition, dependency level, informal care and other personal characteristics.

Costs for the control group and for people in the personal health budget group at baseline were more straightforward: 
Total cost for control group (at each time) = sum of sub-total cost for each service category

Also

Total cost for PHB group at baseline = sum of sub-total cost for each service category

The main analyses used changes in total costs for each person between the amount at baseline and amount at follow-up:

Change in total cost $=$ Total cost at follow-up - Total cost at baseline

Using change controls for any difference in cost between the personal health budget and control groups at baseline before the budget were used and therefore reduce the potential to wrongly conclude that an effect was due to the intervention when it was due to chance. Specifically, this analysis controls for the potential occurrence of participants being recruited at a high service use that subsequently reduces to more normal levels: that is the analysis controls for the potential of 'regression to the mean'. Any impact of using personal health budgets on costs is best indicated by the difference between groups of the change in total costs.

Full details of our costing methodology are available in Appendix C.

\subsubsection{Cost-effectiveness analysis}

To judge the merits of a new intervention like personal health budgets, it is now a standard approach to consider how cost-effectively the intervention would utilise scarce resources (e.g. the NHS and social care budgets) (Drummond, 2005; Weinstein, 1990). Interventions that produce greater effect/benefits for the same cost are favoured over alternatives with poorer cost-effectiveness. Costeffectiveness (CE) ratio can be calculated for this purpose. They are normally expressed in terms of the annual (additional) cost of producing a standardised effect - an improvement in outcomes. Quality-adjusted life years (QALYs) have become the conventional way to measure the effect, a method that allows comparison across interventions that have different types of impact. Any given intervention is therefore evaluated to determine the additional cost of producing, on average, a gain of one QALY.

The approach used in England is to establish a cost-effectiveness threshold, based on the idea that the threshold represents the least cost-effective intervention currently being provided in the NHS (Raftery, 2009; NICE, 2007). In other words, any new intervention with an (incremental) cost per QALY less than this threshold should be implemented. The value of the threshold - expressed as the additional cost to achieve an extra QALY e.g. $£ 20,000$ per QALY gained - can change, increasing when society places a greater value on health (gain) and reducing when there is a lesser willingness to pay for health. This is the approach used by the National Institute for Health and Clinical Excellence 
(NICE). Following convention, the threshold value is denoted by $\lambda$. An intervention (personal health budget) is cost-effective if:

$$
\frac{\Delta C}{\Delta E}<\lambda
$$

In theory, the new intervention would displace a less cost-effective intervention to cover its total cost within the overall budget available (assuming it is reasonably divisible).

Re-arranging the above ratio, we can define the net monetary benefit (NMB) of any intervention as $\Delta N M B=\lambda \Delta E-\Delta C$. A cost-effective intervention is one where the change in NMB is greater than zero:

$$
\Delta N M B=\lambda \Delta E-\Delta C>0
$$

Both the EQ-5D health-related quality of life measure and the ASCOT social care-related quality of life measure, which were included in the study, are preference weighted so that any particular state is valued by respondents in comparison to the implicit value of being dead. NICE are currently operating with a guideline cost-effectiveness threshold for EQ-5D of around $£ 30,000$ per QALY. There is no cost-effectiveness threshold for ASCOT at present, although given the equivalent anchoring to death as for EQ-5D, we might infer a similar threshold value.

\subsubsection{Outcomes, cost-effectiveness and statistical error}

We estimated the impact of personal health budgets using a sample of patients and therefore needed to account for the statistical uncertainty inherent in our measures. To this end, we estimated confidence intervals - i.e. the range of values for the estimate in question which have a $95 \%$ chance of containing the true effect. For the cost-effectiveness analysis we also calculated the probability that estimated NMB was greater than zero for a range of CE threshold $(\lambda)$. We used both parametric and non-parametric approaches, and this is explored further in Appendix $C^{6}{ }^{6}$

\subsubsection{Carers}

This study was organised around the patient, with that patient as the main point of contact. We did not undertake a separate carers' study and instead sampled carers via the cared-for person.

Originally, the cared-for people were asked if we could contact their carer and, if they agreed, a 'permission to contact carer' form was completed. Around 12 months after the consent date we contacted the carer and asked if they would be willing to complete the outcome questionnaire. This

\footnotetext{
${ }^{6}$ It is important to be clear that this statistical uncertainty relative to the 'true effect' is a consequence of sampling and is distinct from any misrepresentation of the true effect of personal health budgets when in routine operation rather than the true effect that applies in the piloting process.
} 
design meant that we only had carer outcomes information at follow-up and for only those study participants who identified a main carer and who also consented for us to contact that carer.

Without a baseline measure for carers, we were unable to use a difference-in-difference estimator. Instead, we followed the approach used for mortality and used a range of (baseline) factors that were anticipated to be correlated with carer outcomes. In this case, the objective was to assess how the use of personal health budgets by the cared-for person impacted on carer outcomes. As such, it was important to include baseline outcomes and need characteristics for the cared-for person in the follow-up estimate of carer outcomes.

\subsection{Qualitative data analysis}

All interviews with organisational representatives, personal health budget holders and carers of budget holders were digitally recorded, with the participants' consent. The completed interviews were transcribed in full.

Analysis of the data followed the framework approach (Ritchie and Spencer, 1994). This involved summarising the data and extracting quotations from each transcript. These summaries were placed in a template organised by themes. The themes were based on the topics included in the interview topic guides. The template enabled the comparison of themes across participants.

\subsection{Challenges to implementing the evaluation design}

As with all new national initiatives, the implementation of personal health budgets presented various challenges, and modifications to the planned evaluation structure and the process had to be made in the course of the study. We have highlighted these challenges as limitations in Chapter 8.

\subsubsection{Demands on organisational representatives working within the pilot sites}

The evaluation team relied heavily on the support from organisational representatives working within pilot sites. Project leads were asked to:

- Organise the recruitment of participants and ensure patients were fully informed about the study;

- Organise baseline outcome interviews to be done within one month of the consent date;

- Organise the completion of the medical record templates at baseline and at 12 months after the consent date;

- Ensure delivery of the personal health budget support plans to the research team.

Furthermore, the research team relied on project leads and organisational representatives to inform them when participants had withdrawn from the study and also they were approached on a monthly basis to clarify whether it remained appropriate to make contact with participants who continued to be involved in the study.

The demands were being made at the same time as organisational representatives were changing how they were delivering health care services. Furthermore, staff were also dealing with significant 
challenges within the pilot sites, such as the abolishment of Strategic Health Authorities and Primary Care Trusts by 2013. These changes would have had an impact on the implementation of personal health budgets within the pilot programme.

\subsubsection{Implementation delays}

A number of implementation delays had a substantial impact on the evaluation. The initial recruitment period was between April 2010 and April 2011. However, challenges in implementing the initiative led to pilot sites not being in a position to begin offering personal health budgets and recruiting to the evaluation. Originally, recruitment was planned to cover the whole period, which would have meant an even spread of participants across the 12 months. However, the delays resulted in the recruitment period being extended to the end of June 2011 to achieve the aim of the study to recruit 3,000 participants.

Chapter 5 reports on the delays in the deployment of personal health budgets. Chapter 6 reports on the impact that recruitment delays and the time lag in the reports of activity becoming available in HES. This delay resulted in not being able to collect secondary service use information for the full 12month follow-up study period for a sample of participants ${ }^{7}$.

\subsubsection{Changing nature of the intervention}

There are reasonable arguments one way and the other about whether evaluations should be conducted on the implementation of new interventions or after some transition period when processes have had a chance to 'settle down'. In this study the former was the case, which meant that the evaluation was of a developing intervention. During the course of the pilot programme and evaluation, some key design features of personal health budgets were being modified and improved locally. This also meant that sites were using a range of methods to implement personal health budgets. The benefit of this, from an evaluation perspective, is that we have the opportunity to compare different models of personal health budget to assess which ones work the best. The downside is the significant extra complexity of evaluating essentially not one but multiple new interventions. The challenges for costing as outlined above were particularly acute as a result.

\subsubsection{Changes to the policy during the evaluation period}

Part of the evaluation is to inform the national roll-out of personal health budgets by providing information about how the initiative should be implemented. During the evaluation period there was a growing emphasis on the roll-out of the initiative, although it was repeatedly highlighted that any decision would be subject to the research findings. On 4 October 2011 the Secretary of State for Health announced that, subject to the evaluation, by April 2014 everyone in receipt of NHS Continuing Healthcare (NHS CHC) will have the right to ask for a personal health budget, including a

\footnotetext{
${ }^{7}$ There is potential that additional analysis could be carried out on the full 12-month period.
} 
direct payment. Within the same time, it is planned that the new Clinical Commissioning Groups (CCGs) will be able to offer personal health budgets on a voluntary basis more widely.

\subsection{Research ethics and the PHBE steering group}

The National Research Ethics Service conferred a favourable ethical opinion for the evaluation. Subsequently, the research was given Research Governance management authorisation to commence the study in each pilot site.

A steering group was set-up that included the full project team and a number of key stakeholders across the pilot sites, including a general practitioner and two service users. The group convened at appropriate times during the evaluation, initially for set up and then to comment on the interim reporting cycle. 


\section{The sample of service users and carers}

\subsection{Summary}

Overall, 2,700 patients were recruited to the evaluation of personal health budget pilot programme, of whom 2,235 were included in the active sample.

The active sample excluded participants:

- for whom we did not receive baseline or follow-up outcome information;

- in residential care at baseline;

- under the age of 18 years of age; and

- who had died before follow-up.

Within the active sample we received:

- 2,168 completed baseline questionnaires: 1,141 in the personal health budget group and 1,027 in the control group;

- 1,341 participants were interviewed for the main follow-up outcome data collection;

- $1,807(81 \%)$ completed baseline medical record templates;

- 1,278 (57\%) completed follow-up medical record templates;

- 1,678 baseline secondary care service use information;

- 1,400 follow-up secondary care service use information;

- 147 carers completed and returned the outcome questionnaire.

Fifty-eight personal health budget holders were interviewed three months after being offered a budget. Fifty-two took part in a follow-up interview, nine months after being offered a budget. Nineteen carers of personal health budget holders were interviewed at three months after the offer of the budget and 13 were interviewed nine-months after the offer.

The chapter provides an overview of the sample characteristics of patients taking part in both the quantitative and qualitative data collection.

\subsection{Introduction}

This chapter introduces the evaluation sample to inform interpretation and understanding of the analysis in Chapters 5 - 7. All results in this chapter were based on the non-imputed dataset.

\subsection{Recruitment, consent and attrition}

\subsubsection{Quantitative data collection}

Figure 3-1 shows the sequencing of the main quantitative and qualitative data collection within the in-depth pilot sites. Study consent was gained from 2,700 people, with 2,235 participants included in the active study sample. The active sample excludes participants for whom we did not receive 
baseline or follow-up outcome information, participants in residential care at baseline ${ }^{8}$, participants under the age of 18 years of age or who had died before follow-up. Within the active study sample, 453 participants withdrew from the study. Of these, 158 (35\%) participants died. In comparison, 31\% $(\mathrm{N}=40)$ of the withdrawn sample in the evaluation of individual budgets pilot programme died during the study period (Glendinning et al., 2008) ${ }^{9}$.

Within the active study sample, we received 2,168 completed baseline questionnaires: 1,141 in the personal health budget group and 1,027 in the control group. Within the active study sample, the fieldwork agency interviewed 1,341 participants (60\% response rate): 663 in the personal health budget group and 678 in the control group.

Within the active sample, the baseline medical record template ${ }^{10}$ was completed for 1,807 ( $\left.81 \%\right)$ participants: 957 in the personal health budget group and 850 in the control group. At 12 months, the medical record template ${ }^{11}$ was completed for 1,278 (57\% of the active sample) participants: 673 in the personal health budget group and 605 in the control group. In terms of extracting secondary care service use data from the hospital episodes statistics database (HES), we received secondary care service use information for 1,678 participants at baseline and 1,400 at follow-up. The lower sample was the result of a number of factors including:

- Participants not giving consent for data to be extracted from the database;

- NHS numbers not being supplied by the pilot site;

- The NHS Information Centre being unable to match the NHS number.

Postal outcome questionnaires were sent out to 282 carers, of which 147 (57\% response rate) were returned. Chapter 7 explores whether personal health budgets had an impact on the caring role and carers' quality of life.

\subsection{Qualitative data collection}

Figure 3-1 shows that 58 budget holders were interviewed around three months after the offer of the personal health budget to discuss their experiences of the process.

Nineteen carers providing assistance to personal health budget holders were also interviewed around three months after the offer of the budget to explore their experiences of the process.

\footnotetext{
${ }^{8}$ Patients in residential care at baseline should not have been invited to participate in the study.

${ }^{9}$ The attrition rate was expected due to the sample of patients participating in the evaluation.

${ }^{10}$ Medical record information collected for the year before consent date.

${ }^{11}$ Medical record information collected for the year after consent date.
} 
Figure 3-1. The sample.

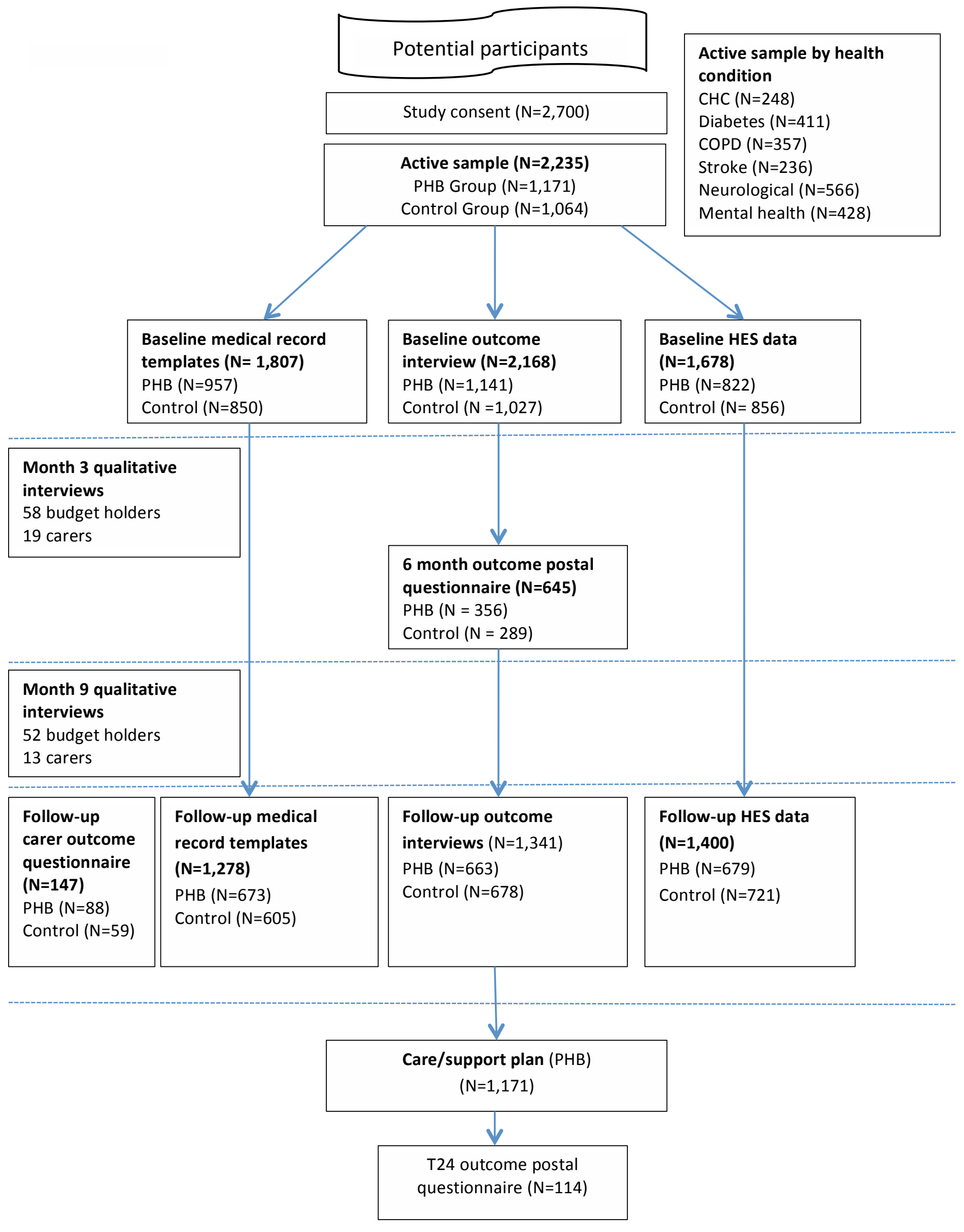




\subsection{An overview of the sample characteristics}

\subsubsection{Completed main outcome questionnaires by health condition}

We gathered information concerning health conditions from participants' medical records. Table 3-1 shows the primary health condition breakdown by personal health budget group and control group at baseline and at 12 months within the active sample.

Table 3-1 Completed main outcome questionnaires by health condition

\begin{tabular}{|ccc}
\hline & Baseline & Main follow-up \\
\hline Personal health budget group & $\mathbf{1 , 1 4 \mathbf { 1 2 } ^ { \mathbf { 2 } }}$ & $\mathbf{6 6 3}$ \\
\hline NHS Continuing Healthcare & 153 & 94 \\
\hline Diabetes & 170 & 97 \\
\hline Stroke & 116 & 71 \\
\hline Mental health & 228 & 105 \\
\hline COPD & 192 & 140 \\
\hline Neurological & 284 & 159 \\
\hline Control group & $\mathbf{1 , 0 2 7}$ & $\mathbf{6 7 8}$ \\
\hline NHS Continuing Healthcare & 86 & 61 \\
\hline Diabetes & 235 & 165 \\
\hline Stroke & 116 & 83 \\
\hline Mental health & 184 & 92 \\
\hline COPD & 152 & 111 \\
\hline Neurological & 262 & 173 \\
\hline
\end{tabular}

Within the medical records, it was reported that overall one per cent of participants ( $n=27)$ had a learning disability and six per cent $(n=132)$ had some form of disability, such as poor mobility, hearing or sight problems. However, we cannot assume that all disability information was included in returned medical record templates.

\subsection{An overview of the sample characteristics}

In determining the validity of subsequent multivariate analysis, it is crucial initially to explore whether the sample within the personal health budget and control groups were similar.

\footnotetext{
${ }^{12}$ The sample for each health condition does not add up to the total overall sample because a group of participants were eligible for NHS Continuing Healthcare and were classified within one of the health condition cohorts.
} 


\subsubsection{Completed baseline outcome questionnaire by age, gender and ethnicity}

Table 3-2 combines information on age, gender and ethnicity and health conditions. The table shows that participants in the personal health budget group were significantly younger (mean $=56$ years) compared to the control group (mean $=61$ years). Overall, $17 \%(n=369)$ of participants were aged 75 years of age or older.

Fifty-three per cent ( $n=588$ ) of the personal health budget sample were female and $52 \%(n=521)$ were female in the control group. Overall, $7 \%(n=150)$ of the sample was from a black and minority ethnic community.

Table 3-2 Completed baseline outcome questionnaire by age, gender and ethnicity

\begin{tabular}{|c|c|c|c|c|}
\hline & Mean age & $\begin{array}{c}75 \text { and over } \\
\%(N)\end{array}$ & $\begin{array}{l}\text { Female } \\
\%(N)\end{array}$ & $\begin{array}{l}\text { BME } \\
\%(N)\end{array}$ \\
\hline Overall & 59 years & 17 (369) & $51(1,109)$ & $7(150)$ \\
\hline PHB Group & 56 years $^{* * *}$ & 15 (167) & $53(588)$ & $7(82)$ \\
\hline $\mathrm{NHS} \mathrm{CHC}$ & 49 years $^{* * *}$ & $13(20)$ & $54(82)$ & $6(9)$ \\
\hline Diabetes & 60 years & $15(25)$ & $53(89)$ & $13(22)$ \\
\hline Stroke & 65 years & $26(30)$ & $41(47)$ & $3(4)$ \\
\hline Mental health & 45 years $^{* * *}$ & $11(24)$ & 49 (111) & $10(22)$ \\
\hline COPD & 66 years $^{* * *}$ & $17(32)$ & 57 (109) & $4(7)$ \\
\hline Neurological & 55 years $^{* * *}$ & $13(36)$ & $53(151)$ & $6(18)$ \\
\hline Control Group & 61 years & $20(202)$ & $52(521)$ & $7(68)$ \\
\hline NHS CHC & 59 years & $21(18)$ & $49(42)$ & $5(4)$ \\
\hline Diabetes & 61 years & $19(44)$ & 52 (123) & $15(36)$ \\
\hline Stroke & 67 years & $31(36)$ & $47(54)$ & $4(5)$ \\
\hline Mental health & 53 years & $10(18)$ & $50(92)$ & $3(6)$ \\
\hline COPD & 70 years & $31(47)$ & $49(74)$ & $0(0)$ \\
\hline Neurological & 61 years & $16(41)$ & 53 (140) & $6(17)$ \\
\hline
\end{tabular}

Table 3-3 shows sexual orientation information from the baseline questionnaire. As the overall sample classified themselves as heterosexual.

Table 3-3 Sexual orientation

\begin{tabular}{lcc}
\hline & PHB Group & Control Group \\
$\mathbf{N}=\mathbf{7 1 9}$ & $\mathbf{N}=\mathbf{7 3 4}$ \\
Heterosexual & $96(687)$ & $95(700)$ \\
Homosexual & $3(21)$ & $3(21)$ \\
Bisexual & $1(10)$ & $2(13)$ \\
\hline
\end{tabular}




\subsubsection{Household composition and informal carers}

Table 3-4 shows that people in the personal health budget group were significantly more likely to be single $(26 \%)$ compared to the control group (19\%) and were more likely to have help from a carer inside $(57 \%)$ or outside $(41 \%)$ the household. People in the control group were significantly more likely to be married (50\%) compared to people in the personal health budget group (45\%).

Table 3-4 Household composition and informal carers

\begin{tabular}{lcc}
\hline & $\begin{array}{c}\text { PHB Group } \\
\%(n)\end{array}$ & $\begin{array}{c}\text { Control Group } \\
\%(n)\end{array}$ \\
\hline Married/cohabiting & $45(506)$ & $50(511)^{*}$ \\
Single & $25(288)$ & $19(196)^{* * *}$ \\
Lives alone & $27(310)$ & $31(314)$ \\
University/college graduate & $27(297)$ & $25(256)$ \\
Secondary school education & $52(572)$ & $55(540)$ \\
Help from a carer inside the household & $57(644)$ & $48(488)^{* * *}$ \\
Help from a carer outside the household & $41(471)$ & $36(373)^{* *}$ \\
\hline Significance levels: ${ }^{*}<0.10^{* *} \mathrm{p}<0.05^{* * *} \mathrm{p}<0.001$ & &
\end{tabular}

\subsubsection{Activities of daily living (ADLs)}

Table 3-5 shows the level of dependency of participants. People in the personal health budget group were significantly more likely to be unable to perform a number of activities of daily living compared to the control group.

Table 3-5 Level of need at baseline

\begin{tabular}{|lcc|}
\hline Unable to.... & $\begin{array}{c}\text { PHB Group } \\
\text { \% (N) }\end{array}$ & $\begin{array}{c}\text { Control Group } \\
\%(\mathbf{N})\end{array}$ \\
\hline get up or down stairs & $29(330)$ & $22(222)^{* * *}$ \\
\hline get outdoors and walk down the road & $32(362)$ & $23(239)^{* * *}$ \\
get around indoors & $14(161)$ & $10(98)^{* *}$ \\
\hline get in or out of bed or chair & $11(128)$ & $6(62)$ \\
use the WC & $13(142)$ & $7(73)$ \\
wash face and hands & $10(108)$ & $6(57)$ \\
\hline bath, shower or wash all over & $12(132)$ & $7(70)$ \\
\hline get dressed/undressed & $10(111)$ & $6(63)$ \\
\hline feed self & $7(83)$ & $4(46)$ \\
\hline
\end{tabular}




\subsection{The sub-sample of qualitative interviews}

\subsubsection{Personal health budget holders}

Fifty eight personal health budget holders were interviewed three months after being offered a budget. Fifty two took part in a follow-up interview nine months after being offered a budget. Table 3-6 gives the number of budget holders interviewed at three and nine months by condition group.

Table 3-6 Number of budget holders interviewed by condition group

\begin{tabular}{lcr}
\hline Condition group & 3-month interview & 9-month interview \\
\hline COPD & $7(12 \%)$ & $6(12 \%)$ \\
Diabetes & $6(10 \%)$ & $5(10 \%)$ \\
Long-term neurological condition & $13(22 \%)$ & $10(19 \%)$ \\
Mental health & $9(16 \%)$ & $8(15 \%)$ \\
NHS Continuing Healthcare & $15(26 \%)$ & $15(29 \%)$ \\
Stroke & $8(14 \%)$ & $8(15 \%)$ \\
Total & $\mathbf{5 8 ( 1 0 0 \% )}$ & $\mathbf{5 2 ( 1 0 0 \% )}$ \\
\hline
\end{tabular}

Table 3-7 shows the gender and age groups of budget holders interviewed.

Table 3-7 Characteristics of budget holders

\begin{tabular}{lcc}
\hline Characteristics & 3-month interview & 9-month interview \\
\hline Gender & $27(47 \%)$ & \\
Male & $31(53 \%)$ & $25(48 \%)$ \\
\hline Female & & $27(52 \%)$ \\
Age group & $1(2 \%)$ & $1(2 \%)$ \\
Under 20 years & $8(14 \%)$ & $7(13 \%)$ \\
$20-29$ years & $11(19 \%)$ & $10(19 \%)$ \\
$30-39$ years & $7(12 \%)$ & $6(12 \%)$ \\
$40-49$ years & $10(17 \%)$ & $9(17 \%)$ \\
$50-59$ years & $13(22 \%)$ & $13(25 \%)$ \\
$60-69$ years & $4(7 \%)$ & $3(6 \%)$ \\
$70-79$ years & $3(5 \%)$ & $2(4 \%)$ \\
$80-89$ years & $1(2 \%)$ & $1(2 \%)$ \\
Not known & $\mathbf{5 8 ( 1 0 0 \% )}$ & $\mathbf{5 2 ( 1 0 0 \% )}$ \\
\hline Total & & \\
\hline
\end{tabular}

The 52 budget holders interviewed at nine months were at different stages with their personal health budgets. Table 3-8 shows that about a fifth had no budget in place at the time of the ninemonth interviews; around two-thirds had had services and support funded through the budget in place for at least three months. 
Table 3-8 Length of time personal health budget in place

\begin{tabular}{lc}
\hline Length of time budget in place & $\begin{array}{c}\text { Number of budget } \\
\text { holders }\end{array}$ \\
\hline No personal health budget in place & $9(17 \%)$ \\
Less than 3 months & $5(10 \%)$ \\
Between 3 and 6 months & $11(21 \%)$ \\
More than 6 months & $25(48 \%)$ \\
Budget terminated by budget holder because health improved & $1(2 \%)$ \\
Budget holder not sure if personal health budget in place & $1(2 \%)$ \\
Total & $\mathbf{5 2 ( 1 0 0 \% )}$ \\
\hline
\end{tabular}

\subsubsection{Carers of personal health budget holders}

The initial aim was to conduct semi-structured interviews with 20 carers of personal health budget holders, at three and nine months after the offer of a budget. Table 3-9 shows that nineteen carers were recruited within the available timeframe. Five of these carers withdrew before the nine-month interview, and a sixth who had been interviewed at three months could not be re-contacted at nine months. The interviews were digitally recorded, with interviewees' consent, and subsequently fully transcribed.

Table 3-9 Number of carers interviewed by condition group

\begin{tabular}{lcc}
\hline Carers who were supporting relatives with: & 3-month interview & 9-month interview \\
\hline Stroke $^{1}$ & 3 & 1 \\
COPD $^{2}$ & 2 & 1 \\
Long-term neurological condition $^{3}$ & 7 & 4 \\
NHS Continuing Healthcare $_{\text {Total }}$ & 7 & 7 \\
\hline
\end{tabular}

1. One person had a long-term neurological condition as well as stroke.

2. One person had multiple health problems, including arthritis, as well as COPD.

3. Two people had additional health problems, including diabetes and Asperger's Syndrome.

Table 3-10 shows the gender and range of ages of interviewees. The carers were, in the main, caring for budget holders eligible for NHS Continuing Healthcare or with long-term neurological conditions. 
Table 3-10 Characteristics of carers interviewed

\begin{tabular}{lcc}
\hline Age group & 3-month interview & 9-month interview \\
\hline Gender & 5 & 3 \\
\hline Male & 14 & 10 \\
\hline Female & & \\
& & \\
\hline Age group & 8 & 4 \\
\hline $40-49$ years & 3 & 3 \\
$50-59$ years & 5 & 4 \\
$60-69$ years & 3 & 2 \\
$70-79$ years & $\mathbf{1 9}$ & $\mathbf{1 3}$ \\
\hline Total & & \\
\hline
\end{tabular}

\subsection{Sample representativeness}

The results in this chapter provide an understanding of the potential bias in terms of both internal and external validity that will need to be controlled for in the difference-in-difference multivariate analysis. In terms of internal validity, the results highlight that there were significant baseline differences between the personal health budget and control groups in terms of dependency levels and socio-demographic factors (such as age, and receiving informal care from someone inside or outside the household). In terms of external validity, the results demonstrate potentially that pilot sites were selecting or excluding specific groups of patients to be put forward for this study which will need to be controlled for in the multivariate analysis. 


\section{The content of the personal health budget support/care plans}

\subsection{Summary}

1,171 care/support plans were received which identified an average of approximately $£ 10,400$ was included in the personal health budgets.

$71 \%(n=828)$ of support/care plans included deployment information; of these around $34 \%$ $(n=283)$ were managed as a direct payment.

Personal health budgets for the NHS Continuing Healthcare cohort had a higher value $(£ 37,418$ per year) compared with the other health condition cohorts such as the stroke cohort $(£ 1,837$ per year). The size of budget will inevitably be linked to the degree of need and may also give an indication of the level of control transferred to the individual.

$53 \%(N=625)$ of budgets were worth less than $f 1,000$, which indicates that pilot sites were either restricting recruitment to patients with less complex needs or were only using personal health budgets to cover specific elements of the care package (for example, well-being support and informal care, or modest health services or equipment).

$47 \%(N=545)$ of personal health budgets were worth more than $f 1,000$, with seven budgets worth more than $£ 150,000$.

\subsection{Introduction}

A number of principles underlying the personal health budget policy were outlined in Chapter 1 (Department of Health, 2009). To recap, personal health budget holders should be:

1. Aware of the level of resources available to them before support planning.

2. Encouraged to develop a support plan which details how the resources will be used to meet identified needs and outcomes. There should be a degree of flexibility on what support and services are paid for through the personal health budget.

3. Given the choice on how the budget is managed. There are three different ways that personal health budgets can be managed (or potentially a combination of them): notionally, where the budget is held by the commissioner but the budget holder is aware of the treatment/service options and their financial implications; managed by a third party; or as a direct payment (in certain approved sites only), where the patient receives a cash payment to buy services.

In this chapter we describe the level of the personal health budget funding, the content of support/care plans, and the degree to which individuals are taking advantage of the flexibility in deciding how to manage the budget and the innovative services/support that can be purchased. We also compare spending patterns within and between health condition cohorts and deployment 
options. Throughout this chapter we will also draw from evidence that has been gathered within the in-depth interviews with budget holders, carers and organisational representatives.

\subsection{Method}

All the information about the level and use of personal health budgets was drawn from the support/care plans. A copy of the care/support plan was requested by the evaluation team for all personal health budget holders participating in the study. Within the active sample, as outlined in Chapter 3, 1,171 care/support plans were received which identified the size of the personal health budget and the request for support/services that would meet the desired outcomes.

\subsection{Size of the personal health budget}

Table 4-1 shows that $53 \%(N=625)$ of budgets were worth less than $£ 1,000$, which indicates that pilot sites were either restricting recruitment to patients with less complex needs or were only using personal health budgets to cover specific elements of the care package (for example, well-being support and informal care, or modest health or social care services or equipment). Chapter 5 will explore whether participants in the 12-month outcome interview felt the level of budget was adequate to meet their needs. However, there was some evidence in the in-depth interviews that were carried out around nine months after the offer of the budget to suggest that budget holders felt that the level was sometimes inadequate (Davidson et al., 2012). It was found that, while most interviewees appreciated the increased choice, control and flexibility of the personal health budget, some thought the benefits had been curtailed by restrictions on what the budget could be used for or lack of services. The lack of flexibility with what services/support could be purchased through the personal health budgets was echoed during the interviews with organisational representatives. A number of pilot sites offered a menu of approved services that budget holders could choose from rather than allowing total service flexibility.

Table 4-1 also shows that $47 \%(\mathrm{~N}=546)$ of personal health budgets were worth more than $f 1,000$, with seven budgets worth more than $£ 150,000$. As highlighted in Chapter 2, for budgets over $£ 1,000$ it was assumed that the resource would be used to substitute for existing services and the money would have been transferred from conventional service budgets. We will explore whether the level of budget had an impact on outcomes and costs in Chapters 5 and 6 .

Table 4-2 shows that, unsurprisingly, people receiving NHS Continuing Healthcare received a significantly higher level of resource (mean $£ 37,418$ ) in their personal health budget compared with the other health condition cohorts. Furthermore, budget holders with a neurological problem also received a higher level of resource (mean $£ 13,055$ per year) compared with the other health conditions, such as people in the stroke cohort who received on average $f 1,837$ per year. The size of budget will inevitably be linked to the degree of need. 
Table 4-1 Size of the personal health budget

\begin{tabular}{|c|c|c|c|c|}
\hline & $\mathbf{N}$ & Mean & Min & Max \\
\hline Overall budget (yearly) & 1,171 & $£ 10,402$ & $£ 0$ & $£ 378,524$ \\
\hline \multicolumn{5}{|l|}{ Budget breakdown } \\
\hline Less than $f 500$ & 359 & $£ 267$ & f0 & $f 500$ \\
\hline$£ 501-£ 1000$ & 266 & $f 680$ & $f 500$ & $£ 1,000$ \\
\hline$£ 1001-f 5000$ & 273 & $£ 2,092$ & $£ 1,000$ & $£ 4,987$ \\
\hline$£ 5001-£ 10,000$ & 67 & $f 6,820$ & $f 5,011$ & f9,996 \\
\hline$£ 10,001-£ 50,000$ & 128 & $£ 25,302$ & $£ 10,067$ & $f 49,904$ \\
\hline$£ 50,001-£ 100,000$ & 55 & $£ 71,554$ & $£ 50,567$ & $f 99,984$ \\
\hline$£ 100,001-f 150,000$ & 16 & $£ 122,172$ & $£ 101,470$ & $£ 147,702$ \\
\hline More than $£ 150,000$ & 7 & $£ 245,294$ & $£ 163,422$ & $£ 378,524$ \\
\hline
\end{tabular}

Table 4-2: Average personal health budget by health condition, demographic and socio-economic status

\begin{tabular}{|c|c|c|c|c|}
\hline & $\mathbf{N}$ & Mean & Min & Max \\
\hline \multicolumn{5}{|l|}{ Health condition } \\
\hline NHS Continuing Healthcare & 155 & $£ 37,418$ & $£ 0$ & $£ 378,524$ \\
\hline Diabetes & 174 & $£ 5,286$ & $£ 1$ & $f 263,970$ \\
\hline COPD & 197 & $£ 3,257$ & f0 & $£ 121,566$ \\
\hline Stroke & 119 & $£ 1,837$ & f1 & $£ 68,171$ \\
\hline Long-term neurological & 295 & $£ 13,055$ & $£ 0$ & $f 308,255$ \\
\hline Mental health & 234 & $£ 3,602$ & $£ 0$ & $£ 92,302$ \\
\hline \multicolumn{5}{|l|}{ Age } \\
\hline Above 75 years of age & 173 & $£ 11,011$ & $£ 0$ & $f 263,251$ \\
\hline Below 75 years of age & 998 & $£ 10,296$ & f0 & $£ 378,524$ \\
\hline \multicolumn{5}{|l|}{ Ethnicity } \\
\hline White population & 1,085 & $£ 10,626$ & f0 & $£ 378,524$ \\
\hline BME population & 86 & $f 7,581$ & f0 & $£ 137,635$ \\
\hline \multicolumn{5}{|l|}{ Benefit receipt } \\
\hline Received benefits & 397 & $£ 8,187$ & f0 & $f 255,212$ \\
\hline No benefits & 774 & $£ 11,538$ & f0 & $£ 378,524$ \\
\hline \multicolumn{5}{|l|}{ Highest education level } \\
\hline \multicolumn{5}{|l|}{ University/college education } \\
\hline Yes & 318 & $£ 14,408$ & $£ 0$ & $£ 378,524$ \\
\hline No & 853 & $£ 8,907$ & $£ 0$ & $£ 308,255$ \\
\hline \multicolumn{5}{|l|}{ Intermediate education } \\
\hline Yes & 171 & $£ 10,945$ & f0 & $£ 223,117$ \\
\hline No & 1,000 & $£ 10,309$ & $£ 0$ & $£ 378,524$ \\
\hline
\end{tabular}




\subsection{Personal health budget deployment mechanisms}

Seventy-one per cent ( $n=828$ ) of support/care plans included deployment information; of these, around $34 \%$ of personal health budgets were being managed as a direct payment $(n=283)$ and $36 \%$ were managed notionally ( $n=298$ ). Despite one of the main aims of the initiative being centred around choice, there is a varying picture between pilot sites as to whether budget holders were given the option to choose the deployment that best met their needs. From the interviews with organisational representatives, only half of the 20 in-depth pilot sites reported that all three deployment options were offered. Five of the pilot sites only offered the notional deployment option to their budget holders. The lack of choice given to budget holders was highlighted to some extent within the in-depth interviews carried out nine-months after the offer of the budget (Davidson et al., 2012). During the interviews, one interviewee reported that she would have preferred it if someone else had managed the budget rather than having a direct payment, but she was not given that option. We will continue to explore the impact of choice and flexibility on outcome and costs in the personal health budget process in Chapters 5 and 6.

Table 4-3 shows that personal health budgets that were managed as a direct payment received a significantly higher level of resource (mean $f 13,712$ ) compared with a notional budget (mean $f 4,523$ )

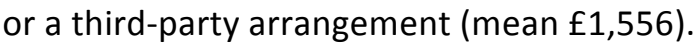

Table 4-3: Description of deployment options by health condition

\begin{tabular}{lcccc}
\hline & DP & Notional & 3rd-party & Combination \\
& Mean (N) & Mean (N) & Mean (N) & Mean (N) \\
Overall & $£ 13,712(283)$ & $£ 4,523(298)$ & $£ 1,556(190)$ & $£ 15,226(57)$ \\
NHS Cont. Healthcare & $£ 38,972(79)$ & $£ 26,216(23)$ & $£ 17,955(9)$ & $£ 47,019(12)$ \\
Diabetes & $£ 998(36)$ & $£ 1,376(43)$ & $£ 517(25)$ & $£ 0(0)$ \\
COPD & $£ 493(31)$ & $£ 1,329(64)$ & $£ 499(38)$ & $£ 2,044(18)$ \\
Stroke & $£ 664(27)$ & $£ 1,602(65)$ & $£ 300(1)$ & $£ 720(1)$ \\
Neurological & $£ 7,851(65)$ & $£ 4,011(89)$ & $£ 1,926(4)$ & $£ 10,325(22)$ \\
Mental health & $£ 6,568(48)$ & $£ 9,969(14)$ & $£ 833(113)$ & $£ 9,741(4)$ \\
\hline
\end{tabular}




\subsection{Patterns of expenditure}

In order to provide an overall picture of the use of personal health budgets, Table 4-4 shows the pattern of expenditure per year included in the personal health budgets. The expenditure was divided into four categories:

- Social care service-related service required to meet both health and social care needs - for example home care, day care, meal services;

- Well-being-related services - for example complementary therapies, leisure, and equipment;

- Therapy and nursing services - for example, nurse and physiotherapy visits;

- Other health services - for example, specialist NHS Continuing Healthcare.

Table 4-4 shows the overall pattern of expenditure.

Table 4-4: Overall patterns of expenditure funded in personal health budgets, whole PHB sample

\begin{tabular}{lllll}
\hline & Mean & \multicolumn{1}{c}{ SD } & Min & \multicolumn{1}{c}{ Max } \\
\hline Total budget & $£ 10,402$ & $£ 28,834$ & $£ 0$ & $£ 378,524$ \\
Social care & $£ 5,712$ & $£ 18,627$ & $£ 0$ & $£ 235,855$ \\
Well-being & $£ 560$ & $£ 1,289$ & $£ 0$ & $£ 23,135$ \\
Therapy & $£ 111$ & $£ 545$ & $£ 0$ & $£ 5,492$ \\
Other health & $£ 1,947$ & $£ 10,226$ & $£ 0$ & $£ 147,207$ \\
\hline
\end{tabular}

A varying picture was uncovered when the level of resource was explored within each health condition cohort. Unsurprisingly, Table 4-5 shows that individuals in the NHS Continuing Healthcare cohort received a higher level of resource for social care-related services such as home care which is

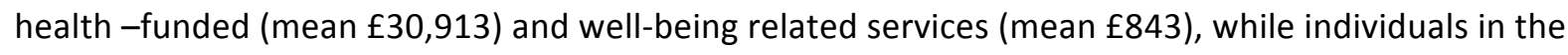
stroke cohort received a higher level of resource for therapy-related services (mean $\mathrm{f358}$ ).

Individuals in the neurological cohort received a significantly higher level of resource for the 'other health service' category (mean $£ 3,901$ ). 
Table 4-5: Overall patterns of expenditure with the personal health budgets

\begin{tabular}{|c|c|c|c|c|c|}
\hline & $\mathbf{N}$ & Mean & SD & Min & Max \\
\hline \multicolumn{6}{|l|}{ NHS CHC } \\
\hline Total Budget & 155 & $£ 37,418$ & $£ 47,672$ & $£ 0$ & $£ 378,524$ \\
\hline Social care & 155 & $£ 30,913$ & $£ 35,274$ & $£ 0$ & $f 235,855$ \\
\hline Well-being & 155 & $£ 843$ & $£ 2,749$ & $£ 0$ & $£ 23,136$ \\
\hline $\begin{array}{l}\text { Therapy and } \\
\text { other nursing }\end{array}$ & 155 & $f 61$ & $£ 472$ & $£ 0$ & $£ 5,492$ \\
\hline Other health & 155 & $£ 2,713$ & $£ 14,351$ & f0 & $£ 123,609$ \\
\hline \multicolumn{6}{|l|}{ Diabetes } \\
\hline Total Budget & 174 & $f 5,286$ & $£ 23,000$ & $f 1$ & $f 263,251$ \\
\hline Social care & 174 & $£ 583$ & $£ 7,495$ & $£ 0$ & $£ 179,790$ \\
\hline Well-being & 174 & $£ 590$ & $£ 520$ & $£ 0$ & $£ 4,103$ \\
\hline $\begin{array}{l}\text { Therapy and } \\
\text { other nursing }\end{array}$ & 174 & $f 29$ & $£ 257$ & $£ 0$ & $f 5,492$ \\
\hline Other health & 174 & $£ 1,978$ & $£ 10,554$ & $£ 0$ & $£ 127,284$ \\
\hline \multicolumn{6}{|l|}{ Stroke } \\
\hline Total Budget & 119 & $£ 1,836$ & $£ 4,162$ & $f 1$ & $f 68,171$ \\
\hline Social care & 119 & f225 & $£ 1,149$ & $£ 0$ & $£ 15,788$ \\
\hline Well-being & 119 & $£ 292$ & $£ 437$ & $£ 0$ & $£ 2,347$ \\
\hline $\begin{array}{l}\text { Therapy and } \\
\text { other nursing }\end{array}$ & 119 & f358 & £914 & $£ 0$ & $f 5,492$ \\
\hline Other health & 119 & $£ 466$ & $£ 1,887$ & $£ 0$ & $£ 33,010$ \\
\hline \multicolumn{6}{|l|}{ Mental health } \\
\hline Total Budget & 234 & $f 3,602$ & $£ 9,663$ & $£ 0$ & $f 92,132$ \\
\hline Social care & 234 & $f 2,198$ & $£ 8,894$ & $£ 0$ & $f 91,950$ \\
\hline Well-being & 234 & $f 598$ & $£ 1,130$ & $£ 0$ & $£ 11,799$ \\
\hline $\begin{array}{l}\text { Therapy and } \\
\text { other nursing }\end{array}$ & 234 & f34 & $£ 244$ & $£ 0$ & $£ 2,533$ \\
\hline Other health & 234 & $£ 374$ & $£ 1,319$ & $£ 0$ & $£ 22,100$ \\
\hline \multicolumn{6}{|l|}{ COPD } \\
\hline Total Budget & 197 & $£ 3,257$ & $£ 12,217$ & $£ 0$ & $f 121,566$ \\
\hline Social care & 197 & $£ 387$ & $£ 3,579$ & $£ 0$ & $£ 102,444$ \\
\hline Well-being & 197 & $f 488$ & f611 & $£ 0$ & $£ 3,557$ \\
\hline $\begin{array}{l}\text { Therapy and } \\
\text { other nursing }\end{array}$ & 197 & $f 65$ & $£ 403$ & $£ 0$ & $£ 5,492$ \\
\hline Other health & 197 & $£ 1,123$ & $£ 5,614$ & $£ 0$ & $f 57,352$ \\
\hline \multicolumn{6}{|l|}{ Neurological } \\
\hline Total Budget & 295 & $£ 13,055$ & $£ 33,414$ & $£ 0$ & $£ 308,255$ \\
\hline Social care & 295 & $£ 4,242$ & $£ 15,225$ & $£ 0$ & $f 123,346$ \\
\hline Well-being & 295 & $£ 585$ & $£ 1,471$ & $£ 0$ & $£ 18,506$ \\
\hline $\begin{array}{l}\text { Therapy and } \\
\text { other nursing }\end{array}$ & 295 & $f 175$ & $£ 700$ & $£ 0$ & $£ 5,492$ \\
\hline Other health & 295 & $£ 3,901$ & $£ 14,501$ & $£ 0$ & $£ 147,201$ \\
\hline
\end{tabular}

Exploring the breakdown of costs, individuals in the NHS Continuing Healthcare cohort received a higher level of resource for social care-related services such as home care which is health-funded for 
this cohort (mean $£ 30,913$ ) and well-being related services (mean $£ 843$ ), while individuals in the stroke cohort received a higher level of resource for therapy-related services (mean $£ 358$ ).

Individuals in the neurological cohort received a significantly higher level of resource for the 'other health service' category (mean $£ 3,901$ ).

Table 4-6 highlights specific services and support that were purchased with the personal health budget. As mentioned above, the majority of the budgets were being spent on social care-related services, although there were signs that budget holders were choosing more innovative services to support their outcomes and needs, such as complementary therapies, leisure and education.

Table 4-6: Specific expenditure examples

\begin{tabular}{|c|c|c|c|}
\hline Social care-related & Well-being related & $\begin{array}{l}\text { Nursing and } \\
\text { Therapy }\end{array}$ & Other health \\
\hline Day centre placements & Complementary therapies & Physiotherapy & Chiropody \\
\hline Respite & Sport membership & $\begin{array}{l}\text { Occupational } \\
\text { therapy }\end{array}$ & Dentistry \\
\hline Home care & Equipment & Counselling & $\begin{array}{l}\text { Psychiatric } \\
\text { appointments }\end{array}$ \\
\hline Meal services & Holidays & Stroke therapy & Specialised $\mathrm{CHC}$ nursing \\
\hline Autism support & Talking therapies & $\begin{array}{l}\text { Nurse } \\
\text { appointments }\end{array}$ & \\
\hline \multirow[t]{3}{*}{ Transport } & Swimming & & \\
\hline & Horse-riding & & \\
\hline & Education & & \\
\hline
\end{tabular}

Note 1: CHC refers to NHS Continuing Healthcare

\subsection{Discussion}

Of significant importance is the finding that around $50 \%$ of the budgets were worth less than $f 1,000$ per year, suggesting that pilot sites were either restricting recruitment to the evaluation to patients with less complex care packages, concentrating on specific elements of packages or providing a small additional resource on top of what would have been conventionally provided. Within this group of budgets, pilot sites may have incurred double running costs that may not be sustainable in the event of a national roll-out of the initiative. It seems that where personal health budgets were sufficiently high enough to substitute for existing services, pilot sites tended to transfer money from conventional service budgets. In terms of the evaluation guiding the roll-out of the initiative, personal health budgets substituting existing services will be of central importance and will be the focus within subsequent multivariate analyses in Chapters 5 and 6.

This chapter also highlighted that there were clear resource differences between the health condition cohorts, deployment options and expenditure patterns. To some extent these differences may reflect the policies within pilot sites rather than decisions made by budget holders. This assumption is supported by the interviews with both organisational staff and budget holders that highlighted varying degrees of choice and control given to budget holders during the personal health budgets process, which will be explored in subsequent multivariate analysis. 


\section{The impact of receiving personal health budgets on outcomes}

\subsection{Summary}

Chapter 5 explores the impact that personal health budgets had on both clinical and subjective outcomes between baseline and the main follow-up 12 month period.

For the clinical outcomes we explored whether personal health budgets had an impact on mortality rates, and health-specific measures for diabetes (HbA1C) and COPD (forced expiratory volume in 1 second-FEV1). For the more subjective measures, we used validated measures: health-related quality of life; care-related quality of life; psychological well-being; and subjective well-being.

To account for possible selection bias we used the difference-in-difference approach.

Key overall findings were:

- Personal health budgets had a significant impact on well-being and quality of life rather than health per se. Although, as the follow-up period was for one year we may not expect that personal health budgets would have an impact on health status. Personal health budgets had a significant positive impact on care-related quality of life, psychological wellbeing and subjective well-being compared to individuals in the control group.

- Personal health budgets had little impact on health status as measured by the clinical effect indicators such as HbA1c and FEV1. Furthermore, personal health budgets didn't have an effect on mortality rates.

- People in the personal health budget group did not report significant improvements in health-related quality of life (EQ-5D) compared to those in the control group.

Key sub-group findings were:

First, there were significant improvements associated with the personal health budget group relative to the control group for:

- COPD sub-group on ASCOT, GHQ-12 and subjective well-being;

- People aged under 75 years of age on ASCOT;

- University or college education on ASCOT and GHQ-12;

- Those not receiving benefits at baseline on ASCOT, GHQ-12 and subjective well-being.

Second, implementation model 4 and 5 had a significant positive impact on outcome change, while model 3 had a significant negative impact.

Third, high-budget personal health budgets $(f 1,000+)$ had a significant positive impact on carerelated quality of life (ASCOT) and psychological well-being (GHQ-12).

\subsection{Introduction}

A key objective of the evaluation was to identify whether personal health budgets improved outcomes from the health and care system for people by giving them greater choice and control over 
the type of support they accessed and the way that support was organised and delivered. The purpose of this chapter is to describe the change in outcomes for individuals in the personal health budget and control groups between baseline (before the intervention began) and 12 months after the consent date (follow-up). Three questions will be addressed in this chapter:

- Is there evidence that personal health budgets lead to better outcomes as compared with conventional service delivery?

- Is there evidence to suggest that specific implementation models lead to comparatively better outcomes for budget holders?

- What other factors are associated with outcome changes?

This chapter is structured as follows. In the next section we briefly review the methods relevant to this chapter that we used to assess the effects of using personal health budgets. Section 5.4 reports the results and we end with a discussion of those results in Section 5.5.

\subsection{Method}

The effectiveness or outcome of an intervention like personal health budgets can be measured in a number of ways. We used clinical outcome measures to assess consequences for health state. A range of subjective measures were used to assess the impact of personal health budgets on healthrelated quality of life and care-related quality of life, and also on subjective global well-being and psychological well-being.

The impact of personal health budgets can also be inferred from peoples' experiences of the process of using the budget and the satisfaction or otherwise they expressed regarding that process. These measures are useful for understanding how well budget holders felt the process was working. There is often a presumption that well-operating processes lead to improved outcomes of personal health budget use compared to alternatives.

\subsubsection{Outcome measures}

\subsubsection{Clinical outcomes}

To explore whether personal health budgets had an impact on health status we collected information from medical records. The template included health-specific measures for diabetes (HbA1c measure) and COPD (forced expiratory volume in 1 second - FEV1). The template can be found in Appendix G.

$\mathrm{HbA1c}$ occurs when haemoglobin joins with glucose in the blood. The more glucose found in the blood, the more glycated haemoglobin ( $\mathrm{HbA1c}$ ) will be present. The HbA1c test is in widespread use 
as a way to check whether diabetes is under control, and current $\mathrm{HbA} 1 \mathrm{c}$ target is between $6.5 \%$ (good control) and $7.5 \%$ (greater risk of hypoglycaemia). ${ }^{13}$

The pulmonary function test (FEV1) is performed to assess lung function and determine the degree of damage to the lungs. FEV1-Forced Expiratory Volume in One Second is the amount of air which can be forcibly exhaled from the lungs in the first second of a forced exhalation. ${ }^{14}$

We also tracked mortality rates in the sample between groups.

\subsubsection{Subjective well-being outcomes}

As described in Chapter 2, we sought to capture some over-arching aspects of well-being by using well-validated global indicators, and also measures that picked up key areas of people's lives, specifically relevant to social care. Chapter 2 and Appendix A describes the measures used in more detail, and here we briefly summarise them.

- Clinical outcomes: The aim was to assess the impact of personal health budgets on the health of study participants in terms of standard clinical markers. To this end, we measured $\mathrm{HbA1c}$ for diabetes sufferers and lung function (forced expiratory volume, FEV1) for COPD sufferers.

- Health-related quality of life: The EQ-5D utility scale aims to measure a person's quality of life in domains likely to be related to their underlying health status. It measures personal functioning (as potentially constrained by poor health). We use the three-level version in this study.

- Care-related quality of life: The care-related quality of life measure (ASCOT) focused on the achievement of everyday activities that might come from the support of services and interventions, as well as from personal functioning.

- Subjective well-being: The measure used in this study seeks to capture general life happiness and satisfaction. We used a scale that considers satisfaction with life, and happiness and satisfaction/worry about the person's health.

The outcome questionnaire also contained a number of socio-demographic and socio-economic questions which will be controlled in the multivariate analysis. Quantitative outcome data was collected on four occasions: at baseline; six months after date of consent; at the main follow-up time (12 months after date of consent); and up to 24 months after date of consent. For the purpose of this chapter, change in outcome measures will be explored between baseline and main follow-up (12months after consent).

\footnotetext{
${ }^{13}$ (http://www.diabetes.org.uk/Professionals/Publications-reports-and-resources/Tools/Changes-to-HbA1c-values/)

${ }^{14}$ (http://copd.about.com/od/glossaryofcopdterms/g/FEV1.htm)
} 


\subsubsection{Process and experience measures}

Interviews with budget holders at three and nine months after the offer of the personal health budget provided us with a further insight into the experiences and impact of the process on individuals and carers that could help explain change in outcome. We asked about their perceptions of the process of using, and their understanding of personal health budgets. We also asked about the overall levels of satisfaction they felt in using a personal health budget.

\subsubsection{Analyses of responses}

As outlined in Chapter 2, we aimed to assess the effect of personal health budgets by measuring whether the average change in the relevant outcome indicator in the personal health budget group was higher than the average change in that indicator for the control group. In other words, relative to the control group, had people in the personal health budget group improved at a faster rate (or declined at a slower rate) on the measured indicator?

In using a difference-in-difference approach, we effectively removed any differences between the groups in the level of the outcome indicator at baseline. So, if one group happened to have higher measured well-being or health status at baseline than the other, this baseline difference will not have a bearing on the follow-up analysis - as we are measuring relative improvement. Whilst this method is an effective way to control for any differences in the characteristics of the personal health budget and control groups affecting the outcome indicator at baseline, there remains a possibility that differences between the groups might cause differences in the rate of change of the indicator, beyond any true effect of PHBs- see Appendix C. A list of all confounding variables explored in the analysis can be found in Appendix $C$.

To safeguard against the possibility of bias of this kind, we also estimated multivariate difference-indifference models. These allow us to identify and remove the effects of differences in baseline characteristics between the groups on any changes in the outcome measures (see also Chapter 6 for an interpretation of these methods). A number of baseline characteristics were explored in these 'controlled' analyses including socio-demographic factors (for example, gender, age, baseline dependency, accommodation, ethnicity), socio-economic factors (for example, education, benefit receipt); and health status (for example, health condition and comorbidities). The possibility of differences in baseline characteristics introduces spurious change bias which is particularly important for mortality rate analysis. Clearly, at baseline there were no differences between the groups in mortality rates (all were alive) but this can mask significant differences in the probability of death immediately after baseline. The confounding variables that contributed to explaining the change on outcomes at follow-up were included in the models.

As anticipated and discussed in Chapter 2 (and Appendix C, section C. 7), in highly complex evaluations such as this one, we will always have at least some missing data for individuals. Where these responses could be considered to be missing at random (to a reasonable extent), we were able to 'impute' values for missing data with techniques that use the underlying patterns in the whole dataset. Appendix $\mathrm{C}$ provides a detailed account of these multiple imputation techniques. The main 
multivariate outcome analyses were run on the imputed datasets. The imputed dataset consists of 2,235 cases, with 1,171 in the personal health budget group and 1,064 in the control group.

The quantitative analyses were also supplemented by evidence from the in-depth qualitative analysis with budget holders at nine months after the offer of the budget and organisational representatives.

\subsubsection{Sub-group effects}

The in-depth interviews with organisational representatives provided us with information around how personal health budgets had been implemented within the in-depth pilot sites and the views held by the staff offering personal health budgets. The interviews also provided the opportunity to classify pilot sites into five different implementation models based on the local processes that were followed during the pilot programme (see Table 1-2).

\subsection{Results}

\subsubsection{Personal health budget process}

The original evaluation design anticipated that all personal health budget holders would be using their budget at the time of the 12-month outcome interview. However, similar to the social care experience within the evaluation of individual budgets pilot programme (Glendinning et al., 2008), the process took significantly longer to be put in place. According to the in-depth interviews with organisational representatives, the delays were caused by various issues including challenges faced within the pilot sites, lack of guidance and the required cultural shift to implement a new way of service delivery.

"Too slowly, too slowly, because of the challenges our PCT is having in terms of administering and assessing for the personal health budgets" (Operational staff member).

"We need training about packages of choice, how to facilitate choice, we don't know this at the minute" (Health professional).

Despite the delays within the system, it was anticipated that a year between consent and follow-up outcome interviews would have been sufficient for the personal health budget process. At the time of the outcome interview, around two-thirds of individuals reported that they had received help paid for by their personal health budget $(65 \%, N=766) .{ }^{15}$ Just under half of people with personal health budgets received help paid for by the budget for more than 6 months $(46 \%, N=544)$. The limited amount of time that budget holders were in receipt of the personal health budget will have an impact on the extent to which the study can explore the impact on longer-term outcomes.

\footnotetext{
${ }^{15}$ These figures are taken from the imputed dataset. In the non-imputed data, $62 \%$ of PHB-holders reported having support in place.
} 
We were able to collect further information on people's view of the personal health budget for the sub-sample of non-imputed data. Among personal health budget holders, around $40 \%(n=217)$ had some understanding of how the resource level was calculated and around 50\% $(n=285)$ were extremely or very satisfied with the support planning process. However, around $15 \%(n=98)$ of individuals in the personal health budget group expressed a degree of dissatisfaction with the support planning process, the knowledge that the support planner had around the initiative and financial arrangements.

The mixed views among budget holders is supported by the findings from the in-depth interviews among budget holders as it was found that, while many people were satisfied with the help and advice that they received in support planning, others did not feel that they had been given a chance to be involved in the process.

However, despite the somewhat negative views held by some budget holders, 38\% $(n=201)$ reported that their view of what could be achieved in their lives had changed a lot and a further $32 \%(N=172)$ reported that it had changed a little. This picture is consistent with the interviews carried out among the sub-sample of budget holders that found a number of participants expressing the view that the personal health budget had been life-changing: by improving their health and outlook on life. For example, one man with a mental health issue said that, in planning for its use, he had been encouraged to focus on what would make him feel better: "I just think it encourages me to look more positively at my health condition than otherwise I would have done".

A few budget holders felt that the personal health budget had given them motivation to do more for themselves to increase their well-being, while others thought that their confidence had improved. However, similar to the in-depth qualitative interviews, a sample of budget holders within the quantitative analysis $(29 \%, \mathrm{~N}=151)$ did not believe that the personal health budget process had changed their view on what could be achieved.

\subsubsection{Variations in clinical outcomes}

\subsubsection{Mortality rates}

Mortality rates after baseline characteristics averaged $8.7 \%$ in the personal health budget group and $6.6 \%$ in the control group, or around $33 \%$ higher in the personal health budget than the control group, but this difference was not statistically significant $(p=0.109)$. As noted above, the case for using control factors is strong for the analysis of mortality. Table 5-1 presents the results of the control estimations and highlight that older and more dependent participants were more likely to die within the study period. The base model compared personal health budget and control groups for the whole sample. An odds ratio ${ }^{16}$ of $+27.1 \%$ greater mortality in the personal health budget group

\footnotetext{
${ }^{16}$ The odd ratios are a measure of effect size and reflect how likely an event (death) will occur.
} 
compared to the control group was estimated, but again not significant $(p=0.252)$. The control factors were strongly significant in accounting for variation in mortality rates after baseline $(F=4.55$, $p<0.001$ ). Specifically, the results suggest that older and more dependent participants (as measured by ADLs scores at baseline) were more likely to die within the study period.

Table 5-1. Mortality rates

\begin{tabular}{|c|c|c|c|c|}
\hline & \multicolumn{2}{|c|}{ Base model } & \multicolumn{2}{|c|}{ Interaction model } \\
\hline & Odds Ratio & Prob & Odds Ratio & Prob \\
\hline PHB Group & 1.271 & 0.252 & 1.213 & 0.426 \\
\hline PHB Group*NHS Continuing Healthcare & & & 1.286 & 0.629 \\
\hline NHS Continuing Healthcare & 1.457 & 0.373 & 1.229 & 0.701 \\
\hline Age & 1.032 & $<0.001^{* * *}$ & 1.032 & $<0.001^{* * *}$ \\
\hline Married & 0.867 & 0.614 & 0.872 & 0.624 \\
\hline Informal care - inside household at baseline & 0.972 & 0.912 & 0.968 & 0.900 \\
\hline Informal care - inside household at baseline & 1.250 & 0.291 & 1.252 & 0.287 \\
\hline ADL score level at baseline & 0.923 & $<0.001^{* * *}$ & 0.923 & $<0.001^{* * *}$ \\
\hline Gender & 1.064 & 0.734 & 1.067 & 0.721 \\
\hline Help with outcome questionnaire & 0.781 & 0.271 & 0.780 & 0.269 \\
\hline Receiving benefits at baseline & 0.810 & 0.415 & 0.813 & 0.421 \\
\hline University or college education & 1.066 & 0.824 & 1.070 & 0.815 \\
\hline Intermediate education & 0.644 & 0.417 & 0.647 & 0.425 \\
\hline BME & 1.019 & 0.965 & 1.025 & 0.955 \\
\hline Health-related quality of life at baseline & 1.383 & 0.625 & 1.400 & 0.614 \\
\hline Psychological well-being at baseline & 0.988 & 0.559 & 0.988 & 0.557 \\
\hline Subjective well-being at baseline & 0.995 & 0.740 & 0.995 & 0.723 \\
\hline Social care-related quality of life at baseline & 1.796 & 0.322 & 1.803 & 0.315 \\
\hline Perceived quality of life at baseline & 1.098 & 0.444 & 1.101 & 0.430 \\
\hline \multicolumn{5}{|l|}{ Perceived health at baseline } \\
\hline Very good & base & & base & \\
\hline Good & 1.006 & 0.994 & 1.012 & 0.988 \\
\hline Fair & 1.639 & 0.532 & 1.646 & 0.529 \\
\hline Bad & 1.633 & 0.566 & 1.646 & 0.561 \\
\hline Very bad & 2.544 & 0.291 & 2.550 & 0.290 \\
\hline Constant & 0.014 & $0.001^{* * *}$ & 0.014 & $0.001^{* * *}$ \\
\hline $\mathrm{N}$ & 2329 & & 2329 & \\
\hline Imputations & 5 & & 5 & \\
\hline
\end{tabular}

Mortality rates were higher overall for the NHS Continuing Healthcare sub-group than the rest of the sample, at $15.43 \%$ overall ( $12.42 \%$ control and $16.97 \%$ personal health budget). The results of the interaction model indicated that the impact of personal health budget use on mortality rates in the NHS Continuing Healthcare cohort was not significantly different from the rest of the sample (and not significant compared to controls in the NHS Continuing Healthcare cohort: $p=0.329$ ). 
Table 5-2 shows that personal health budgets did not a have significant impact on the clinical measures for diabetes and COPD compared to conventional service delivery. Control factors did not have a significant impact on clinical outcome change ( $p=0.755$ for COPD and $p=0.449$ for diabetes). However, due to the one-year follow-up period used in this study, it may be expected that using a personal health budget wouldn't have an impact on health status.

Table 5-2. Change in clinical outcomes between baseline and follow-up

\begin{tabular}{lcc}
\hline & Coef & P>t \\
\hline HbA1c - Diabetes health cohort & -0.481 & 0.449 \\
FEV1 - COPD health cohort & 0.069 & 0.755 \\
\hline Signifance & &
\end{tabular}

Significance levels: ${ }^{*} p<0.10^{* *} p<0.05^{* * *} p<0.001$

\subsubsection{Variations in subjective outcomes}

This section explores whether personal health budgets had a significant impact on subjective outcome measures compared to the control group. A description of the outcome measures used can be found in Chapter 2 and Appendix A.

\subsubsection{Main effect analyses}

On average, the study showed that there were relative improvements in care-related quality of life (ASCOT), psychological well-being (GHQ-12) and subjective well-being for individuals in the personal health budget group compared to those in the control group. Table 5-3 shows baseline and follow-up averages for the listed outcome measures, distinguishing between the personal health budget and control group, and the size of their change without accounting for baseline characteristics. The righthand column reports the differences between the personal health budget and control group, and, in particular, the difference in the change amounts. At baseline, we found significant differences between outcome measures, the size of which had reduced in all cases except EQ-5D by follow-up. 
Table 5-3 Outcome measures - means and differences, baseline and follow-up

\begin{tabular}{|c|c|c|c|c|c|c|}
\hline & & \multicolumn{2}{|c|}{ PHB } & \multicolumn{2}{|c|}{ Control } & \multirow{2}{*}{$\begin{array}{c}\text { Diff } \\
\text { Mean }\end{array}$} \\
\hline & & Mean & SD & Mean & SD & \\
\hline \multirow[t]{3}{*}{ ASCOT } & Baseline & 0.526 & 0.239 & 0.610 & 0.230 & $-0.084^{* * *}$ \\
\hline & Follow-up & 0.584 & 0.239 & 0.628 & 0.235 & $-0.045^{* * *}$ \\
\hline & Change & 0.057 & 0.233 & 0.018 & 0.221 & $0.039^{* * *}$ \\
\hline \multirow[t]{3}{*}{$E Q-5 D$} & Baseline & 0.489 & 0.278 & 0.549 & 0.265 & $-0.059^{* * *}$ \\
\hline & Follow-up & 0.479 & 0.284 & 0.549 & 0.271 & $-0.070^{* * *}$ \\
\hline & Change & -0.011 & 0.221 & 0.000 & 0.207 & $-0.011^{\mathrm{NS}}$ \\
\hline \multirow[t]{3}{*}{ GHQ-12 } & Baseline & 17.274 & 7.480 & 15.549 & 7.267 & $1.726^{* * *}$ \\
\hline & Follow-up & 15.056 & 7.219 & 14.496 & 7.006 & $0.560^{\mathrm{NS}}$ \\
\hline & Change & -2.218 & 7.940 & -1.053 & 7.173 & $-1.165^{* * a}$ \\
\hline \multirow{3}{*}{$\begin{array}{l}\text { Subjective } \\
\text { well-being }\end{array}$} & Baseline & 24.780 & 10.310 & 26.870 & 10.558 & $-2.090^{* * *}$ \\
\hline & Follow-up & 27.336 & 9.724 & 28.293 & 9.913 & $-0.956^{*}$ \\
\hline & Change & 2.556 & 10.017 & 1.423 & 9.654 & $1.133^{*}$ \\
\hline
\end{tabular}

${ }^{\mathrm{a}}$ A negative change denotes an improvement on GHQ-12

Significance levels: ${ }^{*} p<0.10^{* *} p<0.05^{* * *} p<0.001$

However, because we also found significant differences at baseline, the possibility that those differences might account for some of the difference in change, rather than the use of a personal health budget, needed to be considered. For this reason we also estimated the difference in the change over time when controlling for a range of baseline characteristics.

The results are listed in Table 5-4 for ASCOT and EQ-5D and in Table 5-5 for GHQ-12 and subjective well-being. In all cases the control factors were jointly significant at the $10 \%$ level or higher. The introduction of control factors somewhat reduced the difference-in-difference estimates compared to the mean values in Table 5-3 above. For example, rather than an average difference in ASCOT change of 0.039 , the new estimate is reduced to 0.028 . The differences remained statistically significant at $5 \%$ for ASCOT and $10 \%$ for GHQ-12. The subjective well-being measure did not show a significant difference after control factors were introduced.

The difference-in-difference analyses highlight that, after accounting for confounding factors, people in the personal health budget group reported statistically significantly better ASCOT and GHQ-12 outcomes compared with people in the control group. In other words, we found significant positive effects of using personal health budgets on care-related quality of life and psychological well-being. The results highlight that there is good probability that personal health budgets improve people's perceptions of quality of life compared with conventional service delivery.

Despite these positive effects on ASCOT and GHQ-12 outcomes, this result was not repeated for health care-related quality of life (EQ-5D). After accounting for confounding factors, people in the personal health budget group did not report a greater change in health-related quality of life compared to those in the control group. As outlined above, there is both quantitative and qualitative evidence to suggest that personal health budgets did not have an impact on health status when clinical outcomes were explored. 
Exploring the controlling factors, the analysis indicated that improvement in outcome measures was consistently associated with younger participants and baseline dependency levels. The age effect is consistent with the findings from the evaluation of individual budgets (Glendinning et al., 2008), which raised questions about the benefits of IBs for older people. However, the main effects disappeared once we controlled for whether participants were in the personal health budget or control group.

Table 5-4 ASCOT and EQ-5D outcome Difference-in-Difference, personal health budget group, with control factors

\begin{tabular}{|c|c|c|c|c|}
\hline & \multicolumn{2}{|c|}{$\begin{array}{l}\text { Care-related quality of life } \\
\text { (ASCOT) }\end{array}$} & \multicolumn{2}{|c|}{$\begin{array}{l}\text { Health-related quality of life } \\
\text { (EQ-5D) }\end{array}$} \\
\hline & Coeff & Prob & Coeff & Prob \\
\hline PHB group & 0.028 & $0.047^{* *}$ & -0.018 & 0.167 \\
\hline Age & -0.002 & $<0.001^{* * *}$ & -0.001 & $0.023^{* *}$ \\
\hline Male & -0.004 & 0.741 & 0.011 & 0.432 \\
\hline ADL score & $2.11 \mathrm{E}-04$ & 0.813 & -0.004 & $<0.001^{* * *}$ \\
\hline Receives benefits & -0.014 & 0.420 & 0.011 & 0.427 \\
\hline Uni/college educ. & 0.010 & 0.701 & 0.019 & 0.175 \\
\hline Intermediate educ. & -0.004 & 0.840 & 0.022 & 0.198 \\
\hline \multicolumn{5}{|l|}{ Health condition } \\
\hline Continuing Healthcare & 0.009 & 0.656 & -0.074 & $0.001^{* *}$ \\
\hline Stroke & -0.004 & 0.873 & -0.001 & 0.977 \\
\hline Diabetes & 0.044 & 0.146 & $-3.18 \mathrm{E}-04$ & 0.988 \\
\hline Mental health & 0.042 & 0.176 & -0.012 & 0.635 \\
\hline COPD & 0.040 & 0.140 & 0.016 & 0.514 \\
\hline Neurological & 0.043 & 0.215 & -0.022 & 0.298 \\
\hline Follow-up period & 2.90E-04 & 0.319 & 2.95E-05 & 0.889 \\
\hline Consent date & $-2.71 E-05$ & 0.810 & $7.55 \mathrm{E}-05$ & 0.473 \\
\hline Area cost adjust & 0.079 & 0.564 & 0.193 & 0.186 \\
\hline \multicolumn{5}{|l|}{ Area } \\
\hline Town \& Fringe & 0.026 & 0.310 & 0.014 & 0.639 \\
\hline Rural & 0.019 & 0.578 & 0.036 & 0.114 \\
\hline Constant & 0.385 & 0.858 & -1.501 & 0.456 \\
\hline $\mathrm{N}$ & 2235 & & 2235 & \\
\hline Model F & 2.010 & $0.011^{* *}$ & 2.000 & $0.011^{* *}$ \\
\hline Controls - Joint sig & 1.670 & $0.052^{*}$ & 2.110 & $0.008^{* *}$ \\
\hline
\end{tabular}


Table 5-5. GHQ-12 and Subjective well-being Difference-in-Difference, personal health budget group, with control factors

\begin{tabular}{|c|c|c|c|c|}
\hline & \multicolumn{2}{|c|}{$\begin{array}{l}\text { Psychological well-being } \\
\text { (GHQ-12) }\end{array}$} & \multicolumn{2}{|c|}{ Subjective well-being } \\
\hline & Coeff & Prob & Coeff & Prob \\
\hline PHB group & -0.852 & $0.096^{*}$ & 0.762 & 0.213 \\
\hline Age & 0.027 & $0.028^{* *}$ & -0.042 & $0.022^{* *}$ \\
\hline Male & 1.030 & $0.059^{*}$ & -0.669 & 0.110 \\
\hline ADL score & 0.113 & $0.013^{* *}$ & -0.041 & 0.306 \\
\hline Receives benefits & -0.291 & 0.604 & 0.132 & 0.865 \\
\hline Uni/college educ. & -0.334 & 0.561 & 0.446 & 0.457 \\
\hline Intermediate educ. & 0.288 & 0.648 & -0.755 & 0.266 \\
\hline \multicolumn{5}{|l|}{ Health condition } \\
\hline Continuing Healthcare & 1.423 & $0.060^{*}$ & -1.391 & 0.165 \\
\hline Stroke & -1.801 & $0.033^{* *}$ & 0.569 & 0.633 \\
\hline Diabetes & -1.891 & $0.047^{*}$ & 1.563 & 0.101 \\
\hline Mental health & -0.459 & 0.653 & 2.233 & $0.066^{*}$ \\
\hline COPD & -1.278 & 0.136 & 1.141 & 0.350 \\
\hline Neurological & -1.119 & 0.153 & 1.015 & 0.410 \\
\hline Follow-up period & -0.003 & 0.663 & 0.014 & $0.062^{*}$ \\
\hline Consent date & $-1.57 E-04$ & 0.954 & 0.002 & 0.611 \\
\hline Area cost adjust & 1.016 & 0.842 & -0.141 & 0.981 \\
\hline \multicolumn{5}{|l|}{ Area } \\
\hline Town \& Fringe & -0.549 & 0.415 & 0.947 & 0.295 \\
\hline Rural & -1.048 & 0.270 & 1.305 & 0.166 \\
\hline Constant & -1.188 & 0.982 & -45.595 & 0.605 \\
\hline $\mathrm{N}$ & 2235 & & 2235 & \\
\hline Model F & 2.220 & $0.004^{* *}$ & 1.790 & $0.025^{* *}$ \\
\hline Controls - Joint sig & 1.880 & $0.020^{* *}$ & 1.590 & $0.064^{*}$ \\
\hline
\end{tabular}

We can infer from these results that personal health budgets had a significant positive impact on care-related quality of life and psychological well-being. The impact of personal health budgets on well-being/quality of life-related outcomes is not surprising as it was highlighted in Chapter 4 that there were signs budget holders were choosing more innovative well-being services to support their needs that potentially would have an impact on perceptions of quality of life. Furthermore, Chapter 6 will be reporting that well-being services show statistically significant difference between groups in changes over time. The assumption that personal health budgets have an impact on well-being was further supported in the in-depth qualitative interviews with budget holders at nine months after the offer of the budget. Some budget holders felt that the budget had changed their lives by giving them hope for the future, increased social participation, and improved mental health and well-being; "well, if anyone asked what it did for me l'd say it give me my life back; honest, it has changed me completely" (mental health cohort). 


\subsubsection{Sub-group effects}

We anticipated that personal health budgets would have a differential impact on outcome measures for different sub-groups in the sample. In particular, we investigated whether personal health budgets showed significant improvements for the different health conditions, socio-demographic and socio-economic factors and also in terms of the different implementation models for personal health budgets (see Chapter 1). To this end, we estimated controlled difference-in-difference models with sub-group interaction effects.

\subsubsection{Health condition, socio-demographic factors and socio-economic factors}

Table 5-6 reports the (controlled) difference-in-difference results for health condition (analysis run separately for each health condition). The analysis reported in this table compares the outcome indicators for people in this study with a personal health budget against those in the control group by listed health condition. Because sample sizes in these health condition sub-groups were much smaller than for the whole sample, confidence intervals will be wider (or significance probabilities lower), other things equal. To find a significant result in a given health condition sub-group means that the effect size associated with personal health budgets will have had to have been greater in that case than the effect size for the whole sample (at the same significance level). We found significant improvements associated with the personal health budget group relative to the control for the COPD sub-group on GHQ-12 and subjective well-being.

With regard to the implications, with the possible exception of COPD, these results do not suggest that the overall positive effect of personal health budgets is strongly associated with particular health conditions. In that the personal health budget effect seems to be focused around well-being and not health status improvement, it is perhaps unsurprising that health condition is not a strong distinguishing factor. 
Table 5-6. Outcome measures - impact of personal health budgets, by health condition

\begin{tabular}{|c|c|c|}
\hline & Coeff & Prob \\
\hline \multicolumn{3}{|l|}{ NSH Continuing Healthcare } \\
\hline ASCOT & 0.015 & 0.693 \\
\hline$E Q-5 D$ & -0.079 & 0.112 \\
\hline GHQ-12 & -0.117 & 0.906 \\
\hline Subjective well-being & -0.263 & 0.864 \\
\hline \multicolumn{3}{|l|}{ Stroke } \\
\hline ASCOT & -0.015 & 0.619 \\
\hline EQ-5D & -0.018 & 0.544 \\
\hline GHQ-12 & -1.026 & 0.329 \\
\hline Subjective well-being & 1.123 & 0.372 \\
\hline \multicolumn{3}{|l|}{ Diabetes } \\
\hline ASCOT & 0.012 & 0.710 \\
\hline$E Q-5 D$ & -0.015 & 0.533 \\
\hline GHQ-12 & -1.019 & 0.437 \\
\hline Subjective well-being & -0.009 & 0.994 \\
\hline \multicolumn{3}{|l|}{ Mental health } \\
\hline ASCOT & 0.045 & 0.171 \\
\hline EQ-5D & -0.040 & 0.105 \\
\hline GHQ-12 & 0.597 & 0.533 \\
\hline Subjective well-being & 1.255 & 0.289 \\
\hline \multicolumn{3}{|l|}{ COPD } \\
\hline ASCOT & 0.039 & $0.098^{*}$ \\
\hline EQ-5D & 0.001 & 0.959 \\
\hline GHQ-12 & -2.313 & $0.003^{* *}$ \\
\hline Subjective well-being & 2.850 & $0.033^{* *}$ \\
\hline \multicolumn{3}{|l|}{ Neurological } \\
\hline ASCOT & 0.025 & 0.251 \\
\hline EQ-5D & -0.001 & 0.959 \\
\hline GHQ-12 & -0.931 & 0.195 \\
\hline Subjective well-being & -0.128 & 0.901 \\
\hline
\end{tabular}

Significance levels: $p<0.10^{* *} p<0.05^{* * *} p<0.001$

The in-depth interviews with budget holders support the view that, while personal health budgets may provide greater choice and control, clinical health status is more difficult to improve: It certainly helped me to maintain the status quo and, and .. I mean I, I would say it's, it's a positive achievement .. that I have not had any significant deterioration. And that is positive to me... (Long-term neurological cohort).

Turning to age, there was some weak evidence that personal health budgets showed more effect for younger age groups - see Table 5-7. People aged under 75 in the personal health budget group were significantly more likely than people over the age of 75 to report improved care-related quality of life 
(ASCOT) relative to the control group. The same pattern was also evident for the GHQ-12 measure. There was no observed change for over-75s relative to the control group.

Table 5-7.Outcome measures - impact of personal health budgets, by socio-demographic and socio-economic factors

\begin{tabular}{|c|c|c|}
\hline & Coeff & Prob \\
\hline \multicolumn{3}{|l|}{ Over 75} \\
\hline ASCOT & 0.013 & 0.574 \\
\hline EQ-5D & -0.016 & 0.511 \\
\hline GHQ-12 & -0.644 & 0.471 \\
\hline Subjective well-being & 0.722 & 0.548 \\
\hline \multicolumn{3}{|l|}{ Under 75} \\
\hline ASCOT & 0.031 & $0.059^{*}$ \\
\hline$E Q-5 D$ & -0.018 & 0.221 \\
\hline GHQ-12 & -0.891 & $0.091^{*}$ \\
\hline Subjective well-being & 0.770 & 0.250 \\
\hline \multicolumn{3}{|l|}{ University/college education } \\
\hline ASCOT & 0.050 & $0.021^{* *}$ \\
\hline$E Q-5 D$ & -0.030 & 0.148 \\
\hline GHQ-12 & -1.837 & $0.008^{* *}$ \\
\hline Subjective well-being & 1.107 & 0.282 \\
\hline \multicolumn{3}{|l|}{ Intermediate education } \\
\hline ASCOT & -0.002 & 0.937 \\
\hline EQ-5D & -0.035 & 0.324 \\
\hline GHQ-12 & -0.230 & 0.793 \\
\hline Subjective well-being & 0.530 & 0.695 \\
\hline \multicolumn{3}{|l|}{ Receiving benefits } \\
\hline ASCOT & 0.023 & 0.419 \\
\hline EQ-5D & -0.015 & 0.423 \\
\hline GHQ-12 & -0.370 & 0.606 \\
\hline Subjective well-being & -0.075 & 0.944 \\
\hline \multicolumn{3}{|l|}{ Not receiving benefits } \\
\hline ASCOT & 0.030 & $0.087^{*}$ \\
\hline EQ-5D & -0.019 & 0.217 \\
\hline GHQ-12 & -1.054 & $0.088^{*}$ \\
\hline Subjective well-being & 1.112 & $0.089^{*}$ \\
\hline
\end{tabular}

Significance levels: ${ }^{*} p<0.10^{* *} p<0.05^{* * *} p<0.001$ 
People who had a university/college education in the personal health budget group were significantly more likely to report improved care-related quality of life and psychological well-being (GHQ-12) at follow-up relative to the control group.

People not receiving benefits at baseline in the personal health budget group were significantly more likely to report improved care-related quality of life, psychological well-being (GHQ-12) and subjective well-being ${ }^{17}$.

\subsubsection{Implementation models}

A central element of the personal health budget pilot programme was to allow flexibility in how the initiative was implemented within the pilot sites. This flexibility allowed the evaluation to explore what implementation models had a significant impact on outcome change. As reported earlier, five implementation models were developed, based on the findings from the in-depth qualitative interviews with organisational representatives (see Table 1.3). The models were classified in terms of whether pilot sites informed budget holders of the amount in the personal health budget before support planning began; the degree of flexibility in what services/help could be purchased through the budget; and the degree to which different deployment options were offered when the decision on how to manage the budget was made. Table 5-8 reports how they varied across the personal health budget sample. Note that one pilot site could not be classified into any of the four models but had too few cases to form its own model type.

Table 5-8. Personal health budget implementation models

\begin{tabular}{lccc}
\hline Model & $\begin{array}{c}\text { Number of } \\
\text { participants }\end{array}$ & \% PHB group & No of sites \\
\hline Model 1 & 390 & $33.3 \%$ & 8 \\
Model 2 & 283 & $24.2 \%$ & 4 \\
Model 3 & 206 & $17.6 \%$ & 3 \\
Model 4 & 225 & $19.2 \%$ & 4 \\
\hline Other & 67 & $5.7 \%$ & 1 \\
Model 5 (models 1 and 2 together) & 673 & $57.5 \%$ & 12 \\
\hline
\end{tabular}

Table 5-9 shows the extent to which implementation models had an impact on outcome change between the personal health budget and control groups. The main effect analyses considered the average net effect of any personal health budget, whereas this analysis assessed the impact of specific types of budgets (by implementation model). It is clear from the results that the impact of personal health budgets varied significantly according to how they were implemented.

\footnotetext{
${ }^{17}$ The analysis was carried out involving participants from a black and minority ethnic community, though the total numbers in the BME group were below the required 10 per cent level of the whole sample. The effects were not statistically significant.
} 
The analyses highlighted that all implementation models had a significant impact on outcome change. Personal health budgets with model 1 implementation showed a significant effect relative to controls on ASCOT. While personal health budgets with model 2 implementation were associated with better outcomes than controls on GHQ-12. However, both these effects were picked up in model 5 , which combined models 1 and 2 . The common factor between models 1,2 and 4 is the level of choice and flexibility in the services that can be purchased from personal health budgets.

Table 5-9.Outcome measures - impact of personal health budgets, by implementation model

\begin{tabular}{|c|c|c|}
\hline & Coeff & Prob \\
\hline \multicolumn{3}{|l|}{ Model 1} \\
\hline ASCOT & 0.039 & $0.026^{* *}$ \\
\hline$E Q-5 D$ & -0.024 & 0.207 \\
\hline GHQ-12 & -1.052 & 0.130 \\
\hline Subjective well-being & 0.476 & 0.593 \\
\hline \multicolumn{3}{|l|}{ Model 2} \\
\hline ASCOT & 0.037 & 0.161 \\
\hline$E Q-5 D$ & -0.007 & 0.790 \\
\hline GHQ-12 & -1.999 & $0.076^{*}$ \\
\hline Subjective well-being & 0.816 & 0.367 \\
\hline \multicolumn{3}{|l|}{ Model 3} \\
\hline ASCOT & -0.016 & 0.417 \\
\hline EQ-5D & -0.037 & $0.062^{*}$ \\
\hline GHQ-12 & 2.441 & $0.002^{* *}$ \\
\hline Subjective well-being & -1.573 & $0.077^{*}$ \\
\hline \multicolumn{3}{|l|}{ Model 4} \\
\hline ASCOT & 0.044 & $0.027^{* *}$ \\
\hline$E Q-5 D$ & 0.010 & 0.670 \\
\hline GHQ-12 & -2.445 & $0.001^{* *}$ \\
\hline Subjective well-being & 3.680 & $<0.001^{* * *}$ \\
\hline \multicolumn{3}{|c|}{ Model 5 (models 1 and 2 together) } \\
\hline ASCOT & 0.037 & $0.028^{* *}$ \\
\hline$E Q-5 D$ & -0.018 & 0.347 \\
\hline GHQ-12 & -1.384 & $0.073^{*}$ \\
\hline Subjective well-being & 0.623 & 0.396 \\
\hline
\end{tabular}

Significance levels $p<0.10^{* *} p<0.05^{* * *} p<0.001$

In terms of care-related quality of life, people in the personal health budget group among pilot sites following implementation model 5 reported statistically significant better scores than the control group. This finding suggests that there was an impact on quality of life when personal health budgets were being implemented following the basic principles underlying the initiative: that is, budget holders know the resource amount before support planning; there is some degree of flexibility in what services can be purchased; and there is choice in deployment options as to how the budget holder would like the resource to be managed. 
Individuals in the personal health budget group within pilot sites following implementation 4 also reported statistically improved subjective well-being compared to the control group. The only difference between model 4 and 5 is whether the budget holder is informed of the budget level before support planning begins (model 5 ), suggesting possibly that it is the greater choice and flexibility that is more important than knowing the budget level ${ }^{18}$.

This suggestion was echoed by budget holders during the qualitative interviews. We found that personal health budgets had increased the amount of choice and flexibility people had over their healthcare, and that choice was viewed positively. Choice was consistently linked to feelings of greater control over health care which budget holders enjoyed: "I've been able to choose something that I think might be beneficial, whether it transpires to be so or not has yet to be seen. But at least I've been given the opportunity to take control of some of the health care issues available to me. I had the choice". However, the in-depth interviews also indicated that knowing the budget meant that budget holders had a view of whether the resource level was adequate for their needs. The majority of budget holders appeared satisfied because the amount had allowed them to access the services or items they had felt they needed. Budget holders who did not know the budget level could also be unsure whether their budget was enough for their needs: "I think the problem is we don't know how much we've spent".

The implied beneficial effect of choice is underlined by the results regarding model 3 , which, compared to the other models had relatively little flexibility built into the personal health budget process. In this case, we found that the change in EQ-5D, GHQ-12 and subjective well-being was significantly worse among model 3 personal health budget holders than for people in the control group. In other words, personal health budgets had a negative impact in this case. In addition to the lack of flexibility and choice afforded in model 3 , a further explanation of this effect is provided by the qualitative interviews with organisational representatives that hinted at differing attitudes held within pilot sites implementing the different models. Representatives within the pilot sites held both positive and negative attitudes (see appendix D). Some examples of these views are as follows:

"Service users know what is good for them so they know what works and it enables this so I think it is a real positive in terms of control and choice and enabling them to stay in the community and enabling them to come to their own solutions" (Health professional - model 5).

"To be honest, when I've spoken to other people within the health service about someone on the personal health budget, you know, making enquiries, I've received a generally negative attitude towards it. I would say that the majority of other people I've spoken to within the health service aren't keen, they don't think it's a good idea, they don't like it. I've had some people say to me that

\footnotetext{
${ }^{18}$ This assumption could be explored by combining implementation models 1,2 and 4 (implementation model 6). A significant positive impact of personal health budgets was found for care-related quality of life, psychological well-being and subjective well-being within pilot sites following model 6.
} 
they think the whole idea's a disgrace. The people who are negative towards it have come from a lot of different camps. I've spoken to commissioners who don't think it's a good idea, I've spoken to service providers that don't think it's a good idea. I would say that there isn't one particular group that has more of a dislike for it than any other." (Operational staff - model 3).

Another relevant factor in the design of personal health budgets was the amount of the budget. We distinguished high-amount personal health budgets as those which had an annual budget of more than $£ 1,000$, and low-amount PHBs as those which had an annual budget of less than $£ 1,000$. Table 5-10 suggests that the positive impact of personal health budgets was concentrated on those with $£ 1000+$ budgets. This finding could indicate that when a sufficient level of resource is included in the budget to fully cover needs, budget holders feel that they have more choice in the services/support that can be purchased which in turn had an impact on their perceived quality of life at follow-up.

Table 5-10. Outcome measures - impact of personal health budgets, by budget-amount

\begin{tabular}{|ccc|}
\hline & Coeff & Prob \\
\hline High-budget PHB & & \\
\hline ASCOT & 0.032 & $0.046^{* *}$ \\
\hline EQ-5D & -0.024 & 0.229 \\
\hline GHQ-12 & -1.378 & $0.072^{*}$ \\
\hline Subjective well-being & 0.928 & 0.316 \\
\hline Low-budget PHB & & \\
\hline ASCOT & 0.025 & 0.136 \\
\hline EQ-5D & -0.014 & 0.337 \\
\hline GHQ-12 & -0.557 & 0.291 \\
\hline Subjective well-being & 0.666 & 0.359 \\
\hline
\end{tabular}

Significance levels: $\quad p<0.10{ }^{* *} p<0.05^{* * *} p<0.001$

\subsection{Discussion}

A number of key findings have been discussed in this chapter which can be used to guide the national roll-out of personal health budgets. During the study period, and after controlling for baseline differences and health conditions, personal health budgets had a significant positive impact on carerelated quality of life, psychological well-being and subjective well-being compared to individuals in the control group. In terms of socio-demographic characteristics of individuals, the analysis indicated that younger people with personal health budgets had higher ASCOT outcome scores at follow-up than younger people in the control group (after accounting for baseline differences in ASCOT scores between the two groups). There was no significant difference in outcome scores for people over 75 between the groups. We did not find significant differences by age using EQ5D-measured outcomes.

By contrast, personal health budgets had very little impact on health status as measured by the clinical effect indicators such as HbA1c and FEV1. Moreover, there was no significant difference in mortality rates. This finding was further supported by the result that people in the personal health budget group did not report significant change in the health-related quality of life (EQ-5D) compared to those in the control group. This instrument aims to measure a person's quality of life in domains 
that are likely to be related to their underlying health status. Although differences on these measures were statistically insignificant, the average effect in this sample tended to be negative for the personal health budget group compared to control group. The lack of statistical significance means that we cannot reject the conjecture that this result was due to random chance (in terms of the participants that happened to be included in this study), but it might merit further investigation.

We can interpret these findings to mean that personal health budgets impact on well-being and quality of life rather than health per se. Indeed, the benefits in this regard appear to stem from the value people place on increased choice and control in their lives, and the capability this brings for people to improve the more complex or higher-order aspects of their quality of life (see for example, Sen, 1982; Sen, 1993).

This finding is one of the implications for the strong direction of the national roll-out of the initiative after 2012.

A final key message for the personal health budge programme is that outcome change was significantly influenced by how the initiative had been implemented during the pilot programme. Models 4 and 5 had a significant positive impact, while model 3 tended to show negative effects. The findings indicate the following:

1. In some instances, greater flexibility and choice are valued more by budget holders and families than knowing the budget level before support planning begins;

2. The management of cultural change, in terms of acknowledging concerns held by frontline staff, is an important element of the implementation phase that can have an impact on the experience of the personal health budget process and, in turn, on outcomes. One question that was raised within the chapter is whether the success of personal health budget stems from the views held by the staff members implementing the initiative. 


\section{Costs and cost effectiveness}

\subsection{Summary}

In this chapter, we aim to assess whether personal health budgets are cost effective, and this requires us to consider the cost consequences of PHBs as well as their effects on outcomes.

Cost effectiveness is assessed by estimating whether personal health budgets generate greater net benefit than conventional service delivery. Net (monetary) benefit is (value-adjusted) quality of life less the costs of the services people used and this is measured for both personal health budget and control groups.

To account for possible selection bias we used the difference-in-difference approach. We also explored the effect of confounding variables but these were not significant for the cost effectiveness analysis.

Key findings regarding the cost analysis were:

- Services such as primary and secondary care, not covered by personal health budgets (hence 'indirect'), were found to be significantly lower for the personal health budget group compared to the control group after accounting for baseline differences.

- There was no difference in the costs of services that could be directly secured using a personal health budget (such as for nursing, therapy and care services).

- Total costs (direct plus indirect) were not statistically significantly different between the personal health budget and control groups after accounting for baseline differences.

- The cost analyses for health condition sub-samples did not show conclusive differences between personal health budget and control groups.

- Total costs were significantly lower in the group of people with high-value personal health budgets compared to the controls.

Key findings on costs-effectiveness were:

- Measuring net benefits using the care-related quality of life (ASCOT) scale, personal health budgets were found to be cost-effective relative to conventional service delivery at the $90 \%$ confidence level.

- There was no significant difference in the net benefit between the groups when benefit was measured using the health-related quality of life (EQ-5D) scale.

- In the sub-group analyses, personal health budgets produced higher ASCOT-measured net benefits than conditional services for the $\mathrm{CHC}$ and mental health sub-groups (at the $90 \%$ confidence level)

- Otherwise, the health condition sub-group analyses were inconclusive.

- Personal health budgets implemented using model 1 were cost-effective at the $95 \%$ confidence level, as were those with high-value budgets.

- Personal health budgets managed as a direct payment were cost effective at the $90 \%$ confidence level.

- Other socio-economic characteristics showed inconclusive impacts.

Sensitivity analysis supported the main conclusions, in many cases showing personal health budgets to be cost-effective at higher statistical confidence levels. 


\subsection{Introduction}

A range of outcomes were improved for personal health budgets holders compared to the control group as outlined in the previous chapter. In this chapter we aim to assess whether personal health budgets are cost effective, and this requires us to consider the cost consequences of PHBs, as well as their effects on outcomes.

As outlined in Chapter 2, the use of personal health budgets can have potentially beneficial effects in a number of ways. Broadly speaking, we might anticipate that personal health budgets allow recipients: greater choice of what services and support they use to help them manage their condition; to better tailor their care according to their own needs and preferences; and to feel more in control of their own lives and empowered to make decisions for themselves. Furthermore, being given a personal health budget might have implications for the total resources of the care system that people receive.

In these ways, personal health budgets will affect people's quality of life and other outcomes. They will also affect the cost of services and support people use. These cost consequences can be direct in that people change their use of those services and support that can be secured using their personal health budget. Also, the amount of services and support that people can access with their personal health budget, compared to conventional service delivery, will depend on how budgets resourced. In some cases, sites anticipated the costs of conventional services that a person would have used and provided a personal health budget of the same value. In this case, we might expect the cost of services purchased with the personal health budget to be broadly the same as they would have been without the budget - i.e. broadly cost neutral. Other sites, however, used different resource allocation systems that did not necessarily relate directly to the costs of the conventional service the person in question might have used otherwise.

The consequences for costs and expenditure can also be indirect in that the choices people make regarding their budgets and the services and support they receive might change their need for services not covered by their budgets. For example, if a personal health budget allows a person to have greater control and choice to better manage their condition, their need for more intensive health and care services in the future could be reduced. Similarly, by enabling people more scope to act on different priorities than with conventional services, personal health budgets might lead to an increase in the demand for some types of indirect care and support.

In keeping with the usual convention, we focused on recurrent production costs to the health and social care systems. In other words, we do not measure any cost implications of using personal health budgets that fall outside the health and social care system. These additional costs might include family-incurred costs (e.g. from a change in the amount of informal care that personal health budget holders use compared to the control group). We assess the implications for the level of informal care use in Chapter 7, but we do not include the associated cost implications here (in part to maintain consistency with other studies and also because there is little agreement about which costing methodology to use for informal care). Furthermore, we exclude one-off set up costs for personal health budgets; these costs were explored in the $3^{\text {rd }}$ interim report (Jones et al., 2011). Finally, we were unable to measure the 'transaction' costs incurred in commissioning and arranging 
services e.g. in staff time used in the care planning process. Whilst it might have been possible to cost some of this activity for personal health budgets, it would have been very difficult to undertake a like-with-like costing for the care arrangement process for the control group. We would not expect these costs to be significant relative to annual service costs for this population, but, nonetheless, acknowledge that our conclusions could change if transaction costs were included.

The main aims of the chapter are to:

- Develop a consistent framework for assessing the cost and benefits of personal health budgets.

- Report the costs of the services and support used by people in the study.

- Combine these findings with those of the last chapter in order to analyse the costs and benefits of personal health budgets together.

- Analyse how net benefits compared between the personal health budget group and the control group, and therefore assess cost-effectiveness.

- Explore how the cost-effectiveness of personal health budgets varied for different subgroups in the study, such as by health condition, type of budget, budget level and person characteristics.

This chapter is structured as follows. In the next section we briefly describe the methods used to assess cost-effectiveness and then, in the third section, we report the cost analysis. The fourth section has the cost-effectiveness analysis. Section five explores the relationship between costs and benefits. The sixth part of the chapter reports sensitivity analyses. We conclude the chapter with a discussion of the results.

In what follows, the analyses and results are for the imputed dataset unless otherwise stated. The imputed dataset consists of 2,235 cases, with 1,171 in the personal health budget group and 1,064 in the control group.

\subsection{Methods}

\subsubsection{The expected impact of personal health budgets on costs and benefits}

There are many ways in which the use of a personal health budget might produce benefits for the recipient and affect both the service they choose and the costs of those services. Figure 6-1 provides a simple model of the three main effect routes.

- First, we would expect that personal health budgets have a direct (positive) effect on quality of life by giving people more choice and control.

- Second, we would expect that using personal health budgets could lead to a change in the types and intensities of services and support people use. Suppose personal health budgets allow people to better tailor care and support to their own particular needs and preferences. Their health condition and functioning could improve and, in turn, have positive effects on quality of life. There might, conversely, be a negative effect if people make ill-informed choices about their care. 
- A third effect may arise as a consequence of the resource levels provided in personal health budgets. In practice the monetary amount of a personal health budget need not be set at a level that equals the cost of conventional services. Indeed, there are many examples where the monetary value of personal health budgets only partially substitutes for the cost of conventional services. As a result the total public resources available to the person might be lower with a personal health budget than without one. Alternatively, the personal health budget might be provided in addition to conventional services, implying an overall increase in resources to that person. This is a policy decision.

Figure 6-1. The impact of personal health budgets on costs and benefits

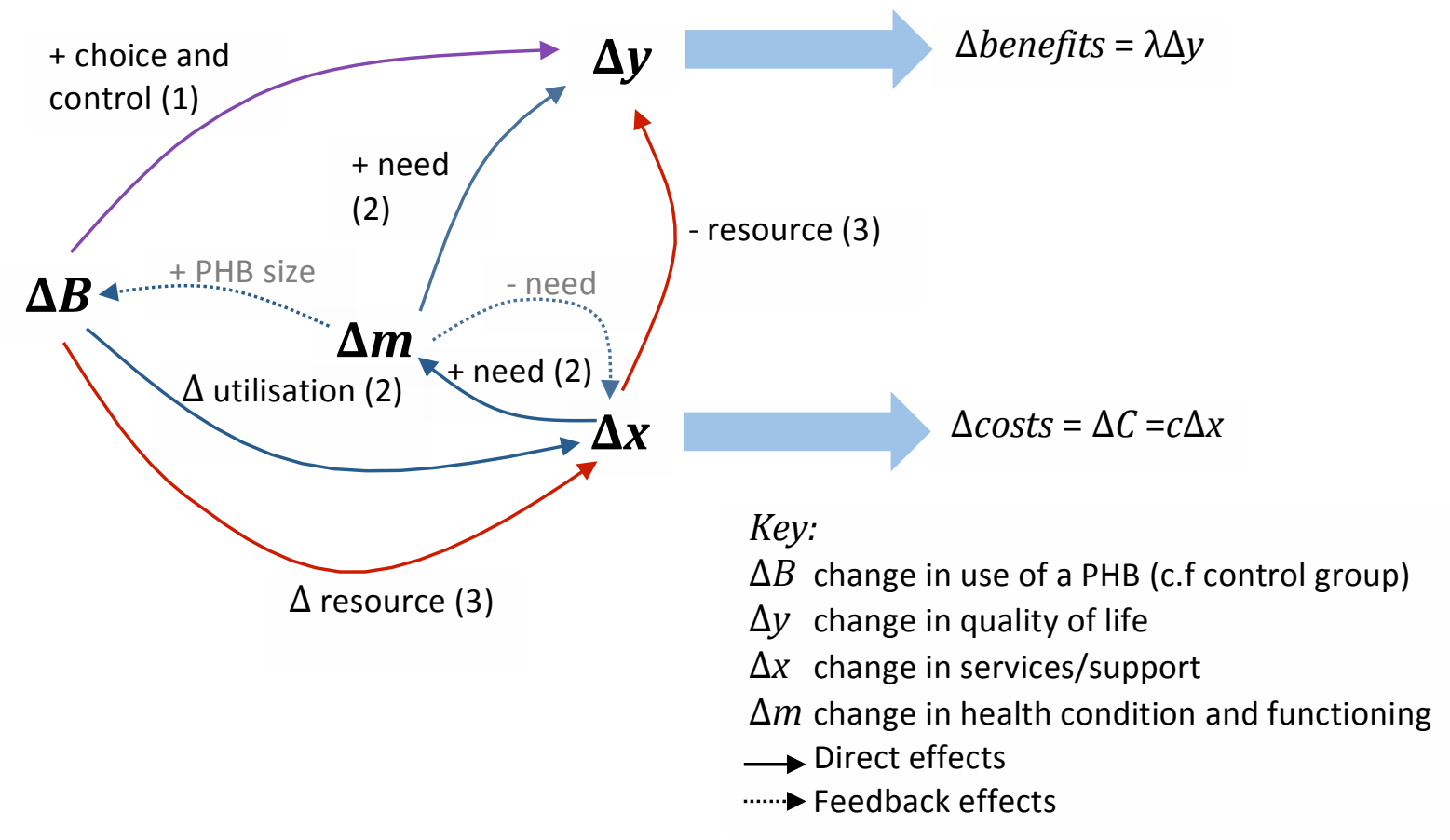

\subsubsection{Assessing costs and cost-effectiveness}

Participants in the study had access to a very wide range of support and services, the majority of which might have been affected, directly or indirectly, by whether the person had a personal health budget. These could include: hospital inpatient, hospital outpatient, accident and emergency, primary care doctors/GPs, practice, community and specialist nurses, a range of therapy services (such as physiotherapy), other continuing health services, community-based social care (including home care, day care, meals, social care personal budgets), social work and respite care. Potentially, study participants might have received equipment and other care-related technology, and many would be using a variety of drug treatments. Personal health budget holders could also use their budget to purchase an array of well-being, exercise and leisure services, equipment and other forms of support in addition to their use of conventional services.

As outlined in Chapter 2, we used a number of data collection methods to gather a comprehensive set of information on service and support use. We also had to develop a methodology to aggregate 
the costs in a way that constituted a like-with-like comparison between personal health budget and control groups, avoided double-counting and was sufficiently comprehensive. The complexity of this task was compounded by the use of different processes between the sites to determine (a) the value of the personal health budget and (b) what services were covered by the personal health budget and what would be provided in the usual way. In many cases, local implementation was developing during the course of the study so, to this end, we made a number of assumptions when combining costs. We tested these assumptions as far as possible, and assessed the implications and sensitivity of the results where different assumptions were made.

Table 2-2 in Chapter 2 gives a broad classification of the services and support being used by people in the study. A key distinction was made between indirect (only) services and direct services, and we follow that approach in this chapter.

\subsubsection{Costing methods}

The activity levels of services measured in the study were assigned a unit cost. Primary and community health care services were given a unit cost using the values outlined in the unit costs for health and social care report published by the Personal Social Services Research Unit (Curtis, 2010; Curtis, 2011). Social care services were given the unit cost using the values outlined in the Personal Social Services Expenditure returns for 2009/2010 and 2010/2011.$^{19}$ Activity data were collected from the outcome interviews for social care and nursing care. GP visits (by type) and allied health professional contacts (e.g. physiotherapy) were collected from GP medical records using the medical record template (see Appendix E). Activity levels were grossed up to an annual amount, representing total activity in the year before baseline and follow-up (12-months after consent date).

Secondary care costs were calculated by applying the appropriate national tariff rates to episodes according to the health research group (HRG) classification of activity (inpatient, outpatient and A\&E tariffs). We also calculated individual lengths of stay for all episodes experienced by study participants in order to apply the long-stay and zero stay payment adjusters in the national tariffs. In the main, the Hospital Episode Statistics (HES) dataset was used to provide activity levels for all study participants who consented for us to download this data. We extracted data for all years after April 2008. In some cases, the last few months of hospital activity were missing due to the time lag in the reports of activity becoming available in $\mathrm{HES}^{20}$ In those instances we were able to fill in the missing data using secondary care information collected from medical records. The detail in the medical record information was more limited than HES so was only used where HES was missing. More information about this method can be found in Appendix C.

\footnotetext{
${ }^{19}$ http://www.ic.nhs.uk/services/social-care/social-care-collections

${ }^{20}$ There is potential that additional analysis could be carried out on the full 12 -month period.
} 
Where consent was granted, requests were made for the care plans for the personal health budget participants to be sent to the research team. These plans included a breakdown of each service or support item and the corresponding cost as met from the personal health budget. We allocated the range of activity into the categories outlined in Chapter 4 . These categories were designed to be comparable to conventional service categories where relevant or to new categories, such as wellbeing services.

In the analysis we use recurrent costs of services and support as used by study participants. We have detailed the implementation costs in interim report 3 (Jones et al., 2011).

\subsubsection{Net monetary benefit}

We used the net (monetary) benefit approach to assess cost-effectiveness. Personal health budgets provide people with benefits, both from the services and support they use and in the way this help is organised. Similarly, people gain benefits from the services and support they are provided under conventional delivery arrangements. These benefits can be measured in a number of ways as outlined in chapter 5 . The net benefit approach usually involves measuring the benefits in terms of the value people associate with having (better) health or care-related quality of life. In this way we can gauge the benefits of personal health budgets compared to conventional service delivery by comparing (the value of) people's quality of life under each option.

As well as benefit implications, the use of personal health budgets affects the cost of services and support people use, as compared to the cost of services and support that would be accrued under conventional service delivery. As there can be both benefit and cost implications, we need a way of combining these effects if we are to assess whether personal health budgets are cost-effective relative to conventional service delivery. The net benefit approach resolves this problem by assessing benefits in $\mathrm{f}$-value terms. This allows a net monetary benefit amount to be calculated by simply subtracting the cost associated with an intervention from the $f$-value of the benefits (i.e. valueadjusted quality of life) it produces.

To make this judgement we need a method to measure the benefits in monetary value terms. An established method is to measure health or care-related quality of life using scales such as EQ-5D or ASCOT and then apply a willingness-to-pay amount for each unit gain on the EQ-5D or ASCOT scales. At present NICE ascribe a willingness-to-pay of between $£ 20,000$ and $£ 30,000$ for each unit gain in EQ-5D - that is, if an intervention improves a person's quality of life by an amount corresponding to being in full health compared to a state that is no better than being dead, over the course of a year, then the value of this improvement in monetary terms is between $£ 20,000$ and $£ 30,000$.

Measures like ASCOT or EQ-5D are amenable scales for this purpose because they are designed to produce quantitative ratings of experiences that people have which are relevant to their quality of life - for example, being in pain, being able to conduct usual activities, feeling in control of their lives etc. These measures place a value on each aspect of quality of life. This value is drawn from a scale that is 'anchored' with reference to the overall value of being in full health as opposed to being dead. Therefore, when we apply a rating from these indicators - e.g. to rate a reduction in someone's sense of control over their life or their level of pain - this rating was made by asking people how far 
they would trade this poorer quality of life for having good quality of life but dying earlier. In other words, these measures aim to allow us to describe the value of quality of life attributes.

Using this approach, we can assess whether personal health budgets are cost-effective by measuring net benefit (i.e. value-adjusted quality of life less service cost) for people in the PHB group and comparing the average with the average net benefit amount measured in the control group. On this basis, if people in the personal health budget group experience greater net benefit than those in the control group, we can infer that personal health budgets are cost effective relative to conventional service options, and should be adopted.

There are two further issues to address, however. First, we need to be sure that the reason we see higher net benefit in the personal health budget group is due to the use of the budget and not something else. Second, we need to be confident that the difference we see in net benefit amounts between the groups is a real difference and not just down to chance in the way the participants in each group were sampled.

As regards the first problem, the two groups - personal health budget and control - may vary in ways other than the use of a personal health budget. This problem is addressed using the difference-indifference design. ${ }^{21}$ If the two groups were different in these other ways, this would show up at baseline before a personal health budget was used. By measuring quality of life and service cost at baseline we can calculate net benefit for each group prior to the use of a personal health budget. Any difference in net benefit between the groups can then be subtracted from the difference in net benefit between the groups at follow-up to remove this selection bias.

The calculation is as follows, assuming a $£ 30,000$ willingness to pay:

NMB diff at baseline $=[€ 30,000 \times$ base QoL(PHB) $-\operatorname{Cost}(\mathrm{PHB})]-[€ 30,000 \times$ base QoL(CG) - Cost(CG)

NMB diff at follow up $=[€ 30,000 \times$ foll QoL(PHB) $-\operatorname{Cost}(\mathrm{PHB})]-[€ 30,000 \times$ foll QoL(CG) $-\operatorname{Cost}(\mathrm{CG})$

The impact of personal health budgets is the difference between these two difference-amounts:

Difference in NMB difference $=$ NMB diff at follow-up - NMB diff at baseline .

A positive value of this difference in NMB difference means that the personal health budgets group experienced greater net benefit than the control group after accounting for any such difference at

\footnotetext{
${ }^{21}$ Regarding this attribution problem, there is always a possibility that the people in the personal health budget group had some characteristics (other than the use of personal health budgets), that systematically differed from those in the control group and which caused the observed difference in NMB or, at least, biased our estimation away from the true value. A fully blind RCT design makes the odds of this happening extremely small. Without a fully randomised design, as in this case, the odds are potentially greater, although a difference-in-difference design minimises the problem by controlling for baseline differences in the sample (see Appendix C).
} 
baseline. By that definition, personal health budgets would be more cost-effective than conventional service arrangements (if the difference was a real difference - see below). In other words, personal health budgets would have generated more benefit after subtracting costs than the conventional approach.

Regarding the second problem, what we have are samples of people using personal health budgets and conventional service delivery. Average values (of net benefits in this case) for each group therefore only imperfectly reflect the true value: they are subject to statistical noise. For this reason we need to determine whether or not the average value of net benefit difference is greater than zero but that the difference is statistically significant.

In the analysis described below we started with a $£ 30,000$ willingness-to-pay threshold and assessed the probability and/or statistical chance that people in the personal health budget group would show a greater improvement in NMB than people in the control group. We used this threshold for valuing both EQ-5D and ASCOT. To be clear, a willingness-to-pay convention for a unit improvement in ASCOT has not yet been fully established. However, as the utility valuation of ASCOT was made on the same basis as for EQ-5D (i.e. both anchored at the being dead-equivalent level) ${ }^{22}$, we expect them to be broadly comparable in value. The impact of personal health budgets on ASCOT and EQ-5D outcome measures was described in Chapter 5.

\subsubsection{Adjusted difference-in-difference}

As outlined in Chapter 2 and applied in the previous chapter on impact - see section 5.3.2 - we used a difference-in-difference approach to account for any baseline differences between personal health budget and control group. Furthermore, to safeguard against possible bias that could arise as a result of differences in the rate of change of costs and benefits between groups, beyond any true effect of personal health budgets, we also estimated multivariate difference-in-difference model with control factors. In this case, unlike the results on impact in the last chapter, we found that control factors made essentially no difference to the results (see below for details). In the multivariate analysis, only confounding factors that contributed to the various models were included. A list of all confounding variables initially explored in the analysis can be found in Appendix C.

\subsection{Costs}

Whole sample

Table 6-1 and Table 6-2 report the cost of services used by the personal health budget and control groups respectively. These tables distinguish costs at baseline and follow-up, and by service category

\footnotetext{
${ }^{22}$ using the same method: a time-trade-off where people exchange longer durations of lower quality of life with short
} periods in full health. 
(see also section 0 for examples of services in these categories). For both groups there was a pattern of increasing direct costs and falling indirect costs over the follow-up period.

Table 6-1. Service and support costs, by type - Personal health budget group, whole sample

\begin{tabular}{|c|c|c|c|c|c|c|c|c|}
\hline & \multicolumn{4}{|c|}{ Follow-up } & \multicolumn{4}{|c|}{ Baseline } \\
\hline & Mean & SD & Min & Max & Mean & SD & Min & Max \\
\hline Social care & 15100 & 23500 & 0 & 250800 & 12800 & 21500 & 0 & 129100 \\
\hline Well-being & 600 & 1300 & 0 & 23100 & 100 & 200 & 0 & 1800 \\
\hline Nursing and therapy services & 200 & 700 & 0 & 5500 & 200 & 500 & 0 & 11000 \\
\hline Other health services & 2100 & 10900 & 0 & 156700 & 2000 & 10400 & 0 & 149800 \\
\hline Sub-total: Direct costs & 18000 & 9100 & 0 & 98400 & 15100 & 11900 & 0 & 137300 \\
\hline Primary care & 740 & 1140 & 0 & 13220 & 680 & 980 & 0 & 10960 \\
\hline Inpatient care & 3010 & 8310 & 0 & 82480 & 5160 & 11430 & 0 & 118200 \\
\hline Outpatient and A\&E care & 800 & 1050 & 0 & 8610 & 930 & 1040 & 0 & 8610 \\
\hline Sub-total: Indirect costs & 4600 & 9100 & 0 & 98400 & 6800 & 11900 & 0 & 137300 \\
\hline Total cost & 22600 & 28800 & 0 & 256000 & 21900 & 29500 & 0 & 218800 \\
\hline
\end{tabular}

Table 6-2. Service and support costs, by type - Control group, whole sample

\begin{tabular}{|c|c|c|c|c|c|c|c|c|}
\hline & \multicolumn{4}{|c|}{ Follow-up } & \multicolumn{4}{|c|}{ Baseline } \\
\hline & Mean & SD & Min & Max & Mean & SD & Min & Max \\
\hline Social care & 11200 & 19300 & 0 & 136900 & 8500 & 17100 & 0 & 124200 \\
\hline Well-being & 100 & 200 & 0 & 1900 & 100 & 200 & 0 & 1900 \\
\hline Nursing and therapy services & 100 & 300 & 0 & 3900 & 100 & 300 & 0 & 4600 \\
\hline Other health services & 2400 & 5100 & 0 & 24200 & 2300 & 4900 & 0 & 23500 \\
\hline Sub-total: Direct costs & 13800 & 7700 & 0 & 89800 & 11000 & 8900 & 0 & 112800 \\
\hline Primary care & 650 & 1020 & 0 & 10580 & 590 & 930 & 0 & 10600 \\
\hline Inpatient care & 2510 & 7030 & 0 & 82480 & 3340 & 8340 & 0 & 102500 \\
\hline Outpatient and A\&E care & 750 & 940 & 0 & 7650 & 850 & 970 & 0 & 11030 \\
\hline Sub-total: Indirect costs & 3900 & 7700 & 0 & 89800 & 4800 & 8900 & 0 & 112800 \\
\hline Total cost & 17700 & 22400 & 0 & 141400 & 15800 & 21800 & 0 & 137800 \\
\hline
\end{tabular}

Expenditure to meet direct costs (social care, well-being, nursing and therapy, and other health services) increased by $25 \%$ over the period for the control group and $20 \%$ for the personal health budget group. Indirect costs, however, fell by $33 \%$ for the personal health budget group compared 
with $18 \%$ for the controls. In both cases the main reduction was for hospital services rather than in primary care (GP services).

The reduction in secondary care costs is not unusual, often being the result of 'regression to the mean': that is, where participants are recruited (meeting the recruitment criteria) at a high ebb of secondary care service-use that subsequently reduces to more normal levels. This feature of the data is a prime reason for designing the study with a control group so that trends can be compared.

Two observations are important in respect of these cost results. First, the control group sample had lower costs on average than the personal health budget group. Comparing the totals, at baseline costs were some $28 \%$ lower for the control group than for the personal health budget group. At follow-up, total costs averaged $22 \%$ lower for the controls. Both of these were statistically significant differences $(p<0.001)$. Second, the mean cost increased at a slightly faster rate in the control group than in the personal health budget group: follow-up costs were $12 \%$ higher, compared to $4 \%$ higher for the personal health budget group.

We can clearly infer that the people in the control group are healthier and have lower care needs than people in the personal health budget group. This result underlines the difficulty in selecting study participants for interventions, like personal health budgets, that are not 'blind' and which are process orientated. In some sites, a non-blind randomisation method for some patient groups was used for study selection (see Chapter 2 for details) (560 of the 2,235 cases were selected using a randomisation process). For the randomised sub-sample, the difference in baseline costs mean values between the two groups was substantially reduced compared to the whole-sample difference, although still significant at the $10 \%$ level. ${ }^{23}$ There was no significant difference in costs at follow-up for the randomisation sub-sample. Due to these anticipated selection issues, we adopted a difference-in-difference design on the basis that changes (in costs) through time are less affected by differences in baseline characteristics. We explore the differences between the randomised and nonrandomised sub-samples as regards to cost-effectiveness below (section 6.5.4).

Costs in both groups showed significant variation and had the usual characteristic of a long righthand tail of the distribution - see Figure 6-2.

\footnotetext{
${ }^{23}$ For a fully double-blind randomised trial we would expect no difference in baseline costs, except by pure chance.
} 
Figure 6-2. Density plot - distribution of total costs, all participants

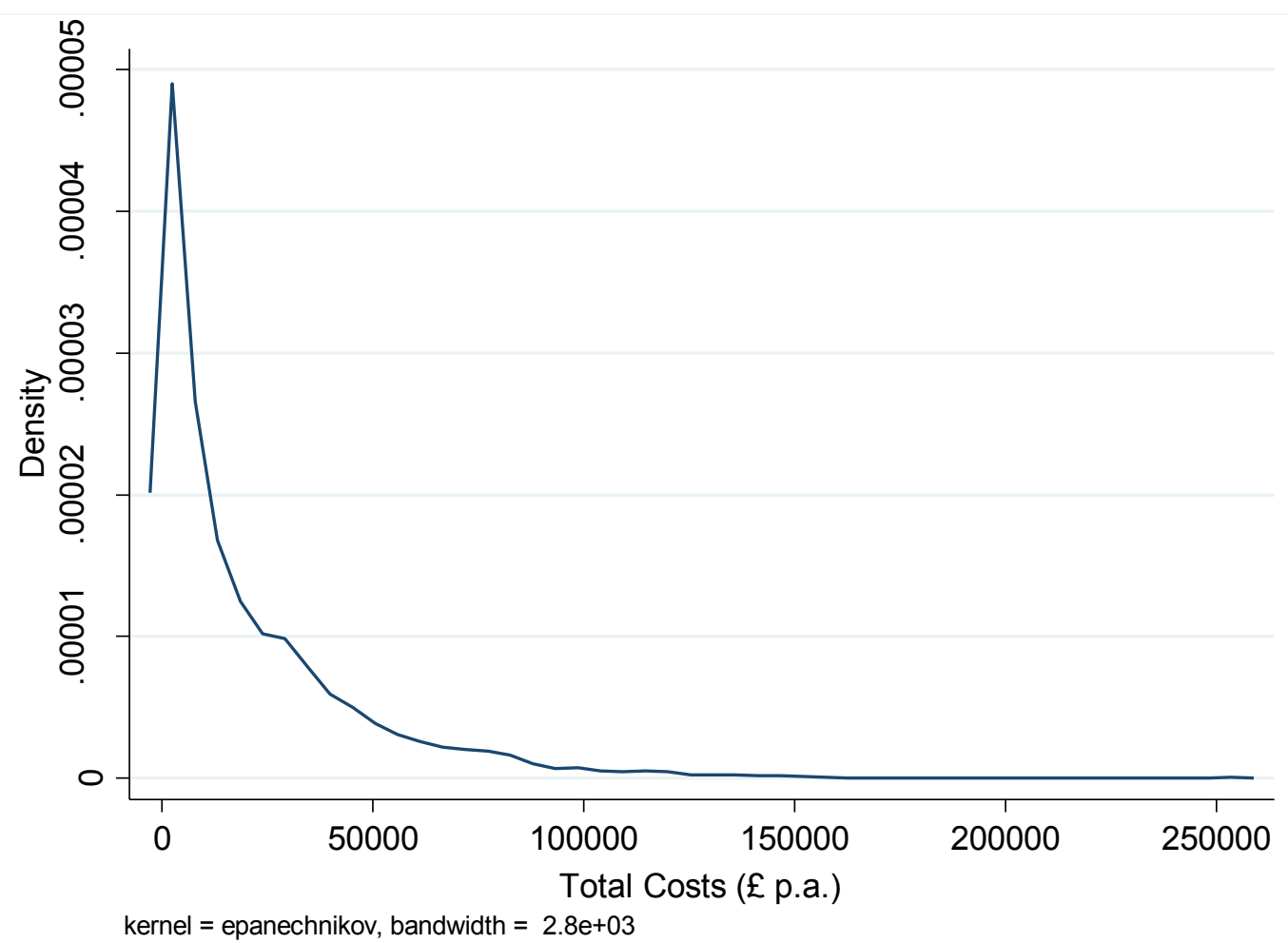

Figure 6-3. Changes in costs and differences - personal health budget and control groups, whole sample

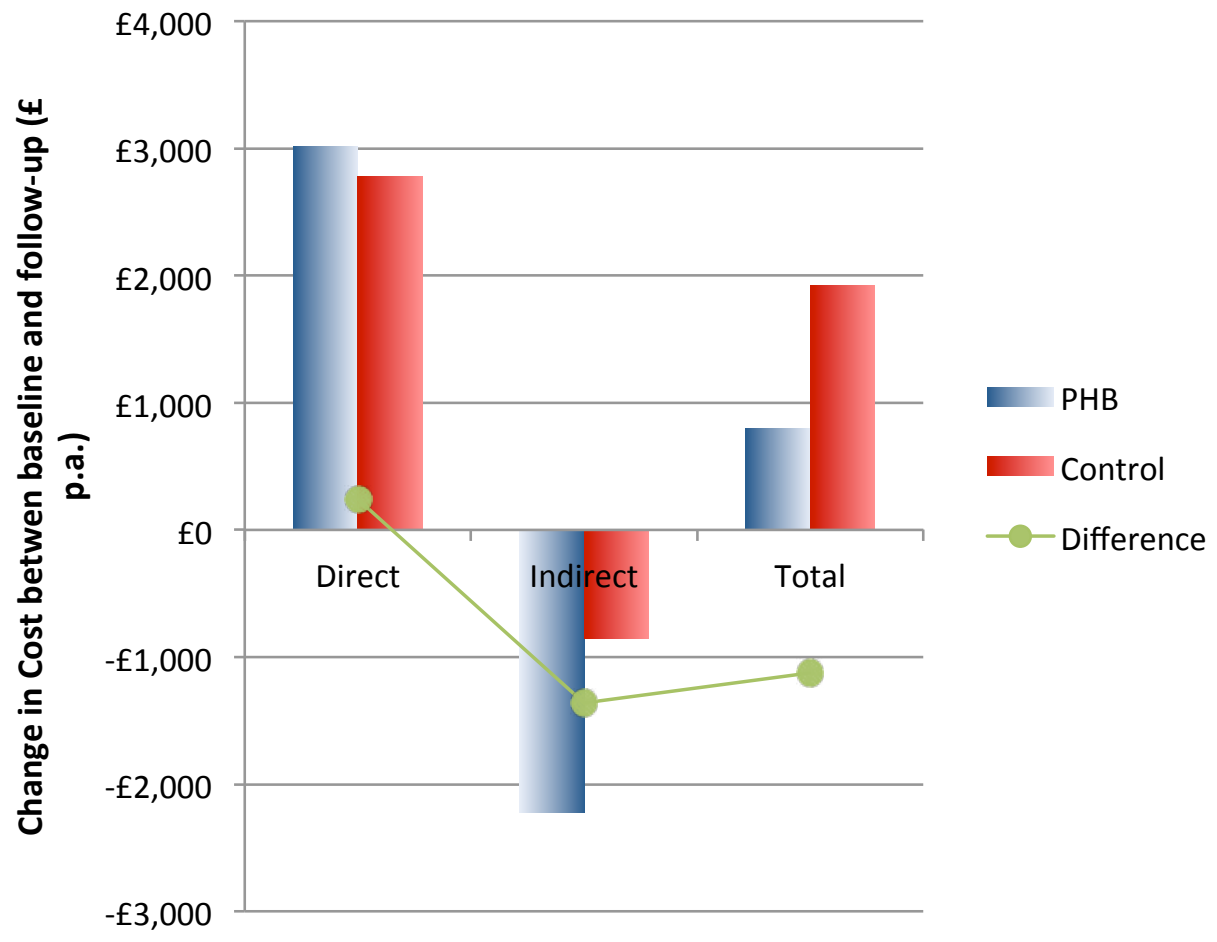


Figure 6-3 above shows the change in cost between baseline and follow-up for both groups and also the differences in these changes between groups through time. Table 6-3 gives more detail. Overall, total costs on average increased by $f 1920$ per person over the study period in the control group and by $f 800$ in the personal health budget group (i.e. a difference-in-difference of $£ 1120$ less for the personal health budget group compared with the controls). Given the variation in total cost differences between the individuals in our sample, we calculated a $95 \%$ confidence interval for this cost difference of $-\mathrm{f} 3440$ to $f 1191$ (i.e. we are $95 \%$ confident that the true cost difference-indifference is in our confidence interval $\left.{ }^{24}\right)$. In this case, the confidence interval includes a zero cost difference so we cannot say that the personal health budget group showed a significantly lower change in costs than the control group.

The faster reduction in indirect costs was the main driver of this overall difference-in-difference result, with indirect costs falling by $£ 1360$ more in the personal health budget group (mainly in respect to inpatient care costs), and this was a significant result. By contrast, direct costs grew at a slightly faster rate in the personal health budget group, with most of this cost growth-difference accounted for by well-being services. This result is in line with expectations and the results reported in Chapter 4 that individuals use their personal health budgets to increase the purchase of well-being type support. We should also be clear that in the majority of cases personal health budgets were provided in addition to usual funding and so constitute an increase in expenditure for the personal health budget group, other things equal. We would, therefore, anticipate that overall cost increases would have been even lower relative to the control group if personal health budgets had been used to substitute for existing service use to a greater degree.

\footnotetext{
${ }^{24}$ Strictly speaking we are saying that there is a $95 \%$ chance that the confidence interval we have calculated hold the true value.
} 
Table 6-3. Differences in service and support costs, by type - whole sample

\begin{tabular}{|c|c|c|c|c|}
\hline & \multicolumn{2}{|c|}{$\begin{array}{l}\text { Change in cost between } \\
\text { baseline and follow-up }\end{array}$} & \multirow{2}{*}{$\begin{array}{l}\text { Difference- } \\
\text { in- } \\
\text { difference }\end{array}$} & \multirow[t]{2}{*}{$\begin{array}{l}\text { Significance } \\
\text { probability }\end{array}$} \\
\hline & PHB & Control & & \\
\hline Social care & 2310 & 2720 & -400 & 0.635 \\
\hline Well-being & 500 & 0 & 510 & $<0.001^{* * *}$ \\
\hline Nursing and therapy services & 80 & -10 & 90 & 0.109 \\
\hline Other health services & 120 & 70 & 50 & $0.003^{* *}$ \\
\hline Sub-total: Direct costs & 3020 & 2780 & 240 & 0.759 \\
\hline Primary care & 60 & 70 & -10 & 0.830 \\
\hline Inpatient care & -2150 & -830 & -1320 & $0.040^{* *}$ \\
\hline Outpatient and A\&E & -130 & -100 & -30 & 0.427 \\
\hline Sub-total: Indirect costs & -2220 & -860 & -1360 & $0.042^{* *}$ \\
\hline Total cost & 800 & 1920 & -1120 & 0.319 \\
\hline
\end{tabular}

Significance levels: $p<0.10^{* *} p<0.05^{* * *} p<0.001$

$N=2235$

To sum up, statistically significant differences between the groups in changes in costs over time were found for inpatient care, well-being and other health services. The total cost difference-in-difference was not significantly different, however, for the whole sample.

\subsubsection{Costs of services by personal health budget type}

Just under $45 \%$ of personal health budgets in the study were deployed as direct payments (as opposed to notional budgets or using third parties). The average budget value for recipients with only direct payment personal health budgets was $£ 12880$ compared to an average of $£ 8330$ for all types of personal health budget. In terms of expenditure made from budgets, total expenditure from direct payment budgets in the study $(£ 6.62 \mathrm{~m})^{25}$ accounted for approximately two-thirds of the total expenditure made from all types of personal health budgets $(f 9.75 \mathrm{~m})$. To put these figures in context, taking just personal health budget holders, expenditure from direct payment personal health budgets accounted for $31 \%$ of direct expenditure totals at follow-up and $25 \%$ of total expenditure - see Table 6-1.

Generally speaking we would expect expenditure from direct payment personal health budgets to be used to fund services and support provided by the non-public (or independent) sector. ${ }^{26}$ This

\footnotetext{
${ }^{25}$ In other words, an average of $₫ 5650$ over the 1171 personal health budget holders.

${ }^{26}$ We make this assumption because in theory the commissioning of publicly-delivered services would not require a monetary payment. Nonetheless, it might be possible that local arrangements are made to accommodate these sorts of transactions e.g. netting off the cost of public services from the direct payment. These arrangements ought to be classed as mixed notional and DP deployment in the data, but interpretation can differ between sites.
} 
assumption underpinned an estimation of the change in the amount of service secured outside the NHS (and in-house social care providers) as a result of the introduction of personal health budgets. Again we estimated this outsourcing figure using a difference-in-difference approach. Irrespective of the use of personal health budgets, around $90 \%$ of social care is purchased from independent sector providers anyway. Furthermore, well-being services are not (generally) provided by the NHS. For this analysis we therefore assumed that all well-being services secured at follow-up were outsourced. Of the remaining services categorised as direct costs, e.g. therapy services, we assumed that where these services were funded using a direct payment they were secured externally, and otherwise they were supplied by internal providers (i.e. NHS and in-house care providers).

These assumptions mean that at baseline, for both the control and personal health budget groups, all direct expenditure went to internal providers except for the $90 \%$ of social care that was secured externally. At follow-up the same applied for the control group. However, for the personal health budget group all well-being services and $90 \%$ of social care expenditure were sourced externally. Furthermore, the remaining direct expenditure (after well-being service and $90 \%$ of social care expenditure is removed) was externally sourced if the person had a direct payment personal health budget.

To reiterate the amount of care secured from external providers was:

\begin{tabular}{|c|c|c|}
\hline Group & Baseline & Follow-up \\
\hline Control & $\begin{array}{l}90 \% \text { of social care } \\
\text { expenditure }(\mathrm{SC}) \text { at } \\
\text { baseline }(\mathrm{A})\end{array}$ & $\begin{array}{l}90 \% \text { of SC at follow-up } \\
\text { (B) }\end{array}$ \\
\hline Personal health budget & $\begin{array}{l}90 \% \text { of SC at baseline } \\
\text { (C) }\end{array}$ & $\begin{array}{l}90 \% \text { of } S C \text { at follow-up + well-being } \\
(W B)+\text { all other direct expenditure } \\
\text { by a } D P^{27} \\
\text { (D) }\end{array}$ \\
\hline
\end{tabular}

The difference-in-difference is $=($ Cell $D-$ Cell $C)-($ Cell B - Cell A $)$.

This difference-in-difference was calculated to be $£ 2180$ per year for the sample. It was our estimate of the amount of expenditure going outside the NHS following the use of personal health budgets. ${ }^{28}$ This amount was equivalent to just over $12 \%$ of the $f 18000$ total direct expenditure in the personal health budget group.

\footnotetext{
${ }^{27}$ This call is equal to: (Direct expenditure at follow-up - 90\% of social care expenditure at follow-up) $\times$ DP\% + well-being expenditure at follow-up $\times(1-$ DP\% $)+90 \%$ of SC at follow-up.

${ }^{28}$ This value is significant at the $90 \%$ confidence level.
} 
Our definition of social care in this study was inclusive and could incorporate services that might be provided by the NHS for people without personal health budgets. This would mean less expenditure was externally sourced before the introduction of personal health budgets and consequently the change would be greater following their introduction. Re-calculating on the basis that $80 \%$ of social care was provided by the independent sector increased the change figure to $f 3110$ or around $17 \%$ of direct expenditure. ${ }^{29}$

This outsourcing analysis gives some indication of the scale outsourcing associated with the use of personal health budgets, but it relies on the assumptions detailed above. Different assumptions would produce different results. We might have assumed that therapy and other health services funded under notional personal health budgets could have been commissioned from external providers. Also, we assumed that all well-being expenditure went externally, but this might not be the case. For example, if we assume that only half of well-being expenditure actually went externally, then the impact of personal health budgets would be in the order of $f 1900$ per year for the sample. The wider implications of these results are considered in chapter 9.

\subsubsection{Costs for sub-groups}

We can further explore costs difference by sub-samples of the study population. The following tables present changes in costs for the different health condition groups, by different cost category. Table 6-4 shows the change to indirect service use for each health condition group. In this case, the personal health budget group reduced their expenditure on indirect services to a greater degree than the control group. The differences in the changes through time were significant for the mental health and NHS Continuing Healthcare cohorts. The result for the NHS Continuing Healthcare cohort was consistent with the views held by project leads within the in-depth qualitative interviews. It was consistently thought the personal health budgets would lead to cost-savings within this cohort.

Changes in direct costs are shown in Table 6-5. There were no significant differences between the personal health budget and control group in these cost changes. We should note, however, that statistical precision may be limited by the relatively small sample sizes of each of the six health conditions.

Table 6-6 reports the changes in the total costs for each group, which is the sum of indirect and direct costs. Overall, the difference in the change over time (third column) was not significantly different from zero for any of the health conditions. For the NHS Continuing Healthcare and mental health cohorts, the high variance in direct costs in the sub-samples diluted the precision of the result for indirect costs. Not finding significant total cost differences does not remove the significance of the difference regarding indirect costs.

\footnotetext{
${ }^{29}$ This difference is significant at the $95 \%$ confidence level.
} 
Table 6-4. Changes in indirect cost between baseline and follow-up, by patient group

\begin{tabular}{l|r|r|rrrr}
\hline & \multicolumn{1}{l}{$\begin{array}{l}\text { PHB } \\
\text { Change }\end{array}$} & $\begin{array}{l}\text { Control } \\
\text { Change }\end{array}$ & Difference & & & \\
& Change & \multicolumn{1}{l}{ Prob } & Cl+ & \multicolumn{1}{c}{$\mathrm{Cl}-$} \\
\hline Diabetes & -1730 & -410 & -1320 & 0.263 & -3730 & 1090 \\
Stroke & -4940 & -4130 & -810 & 0.605 & -3880 & 2280 \\
Mental health & -2980 & 70 & -3050 & $0.008^{* *}$ & -5290 & -820 \\
COPD & -930 & 670 & -1600 & 0.109 & -3560 & 370 \\
Neurological & -1240 & -1150 & -90 & 0.921 & -2060 & 1870 \\
CHC & -4570 & -530 & -4040 & $0.058^{*}$ & -8220 & 140 \\
\hline
\end{tabular}

Note 1: $\mathrm{CHC}$ refers to NHS Continuing Healthcare

Significance levels: ${ }^{*} p<0.10^{* *} p<0.05^{* * *} p<0.001$

Table 6-5. Changes in direct cost between baseline and follow-up, by patient group

\begin{tabular}{l|r:r:rrrr}
\hline & \multicolumn{1}{l}{ PHB } & Control & Difference & & \\
& Change & Change & Change & Prob & Cl+ & \multicolumn{2}{c}{ Cl- } \\
\hline Diabetes & 3610 & 1900 & 1710 & 0.197 & -900 & 4320 \\
Stroke & 1900 & 4740 & -2840 & 0.146 & -6680 & 1000 \\
Mental health & 980 & 800 & 180 & 0.921 & -3630 & 4000 \\
COPD & 4230 & 2680 & 1550 & 0.268 & -1260 & 4360 \\
Neurological & 4620 & 3410 & 1210 & 0.411 & -1690 & 4110 \\
CHC & 860 & 5450 & -4590 & 0.301 & -13560 & 4390 \\
\hline
\end{tabular}

Note 1: $\mathrm{CHC}$ refers to NHS Continuing Healthcare

Significance levels: ${ }^{*} p<0.10^{* *} p<0.05^{* * *} p<0.001$

Table 6-6. Changes in total cost between baseline and follow-up, by patient group

\begin{tabular}{|c|c|c|c|c|c|c|}
\hline & \multirow{2}{*}{$\begin{array}{l}\text { PHB } \\
\text { Change }\end{array}$} & \multirow{2}{*}{$\begin{array}{l}\text { Control } \\
\text { Change }\end{array}$} & \multicolumn{4}{|c|}{ Difference } \\
\hline & & & Change & Prob & $\mathrm{Cl}+$ & $\mathrm{Cl}-$ \\
\hline Diabetes & 1880 & 1490 & 390 & 0.832 & -3320 & 4100 \\
\hline Stroke & -3040 & 600 & -3640 & 0.146 & -8570 & 1290 \\
\hline Mental health & -2010 & 870 & -2880 & 0.199 & -7390 & 1640 \\
\hline COPD & 3300 & 3350 & -50 & 0.979 & -3780 & 3680 \\
\hline Neurological & 3380 & 2260 & 1120 & 0.506 & -2180 & 4410 \\
\hline $\mathrm{CHC}$ & -3710 & 4920 & -8630 & 0.112 & -19520 & 2270 \\
\hline
\end{tabular}

Note 1: $\mathrm{CHC}$ refers to NHS Continuing Healthcare

Significance levels: ${ }^{*} p<0.10^{* *} p<0.05^{* * *} p<0.001$

Another important distinction in the sample is by age. People aged over 75 are known to be more intensive users of health and social care services than younger people. At baseline in this study, people aged over 75 had $24 \%$ higher total costs than people aged 75 or less $(p=0.016)$. With a higher capacity to benefit from cost-saving interventions, we might expect to see differences in the change in cost through time according to age group.

Table 6-7 has the difference-in-difference results for people aged over 75 and, as can be seen, the personal health budget group had lower growth in total cost than the control groups by $-£ 4300$, with 
much of this difference due to the relative reductions in inpatient costs. This was a significant difference at the $10 \%$ confidence level. $^{30}$

Table 6-7. Differences in service and support costs, by type - over-75 sample

\begin{tabular}{|c|c|c|c|c|c|c|}
\hline & \multicolumn{2}{|c|}{ Baseline cost totals } & \multicolumn{2}{|c|}{$\begin{array}{l}\text { Change in cost between } \\
\text { baseline and follow-up }\end{array}$} & \multirow{2}{*}{$\begin{array}{l}\text { Difference- } \\
\text { in- } \\
\text { difference }\end{array}$} & \multirow{2}{*}{$\begin{array}{l}\text { Significance } \\
\text { probability }\end{array}$} \\
\hline & PHB & Control & PHB & Control & & \\
\hline Social care & 13530 & 9060 & 1370 & 3240 & -1880 & 0.364 \\
\hline Well-being & 60 & 40 & 300 & 0 & 300 & $<0.001^{* * *}$ \\
\hline Nursing and therapy & 160 & 120 & 230 & 40 & 180 & 0.210 \\
\hline Other health services & 2190 & 3340 & 140 & 110 & 30 & 0.525 \\
\hline Sub-total: Direct costs & 15940 & 12560 & 2030 & 3390 & -1360 & 0.449 \\
\hline Primary care & 890 & 760 & -60 & -30 & -30 & 0.804 \\
\hline Inpatient care & 7890 & 5540 & -3710 & -1110 & -2600 & 0.152 \\
\hline Outpatient and A\&E & 1070 & 880 & -360 & -50 & -310 & 0.152 \\
\hline Sub-total: Indirect costs & 9840 & 7180 & -4130 & -1190 & -2940 & 0.124 \\
\hline Total cost & 25780 & 19740 & -2100 & 2200 & -4300 & $0.062^{*}$ \\
\hline
\end{tabular}

As outlined in Chapter 4, the value within personal health budgets varied considerably among the sample. We made a qualitative distinction between budgets of less than and greater than $£ 1000$ per annum - see Chapter 2 and Appendix C for details. Just under $40 \%$ of personal health budget holders in the active (imputed) sample had 'high' budgets in excess of $£ 1000$ p.a. In the NHS Continuing Healthcare cohort over $80 \%$ had $£ 1000+$ budgets, with the proportion below $45 \%$ for the other groups. We have assumed that high budget amounts indicate that the personal health budget was funded in place of existing service delivery.

We can also compare costs for people in the personal health budget cohort with different types of personal health budget. In particular, we can compare those people with high-value budgets (i.e. those worth more than $f 1000$ per annum) and those with low-value budgets. In regard to our assumptions about costing, in the latter case personal health budgets were provided in addition to usual services, whilst for the former the personal health budget would substitute for conventional services.

\footnotetext{
${ }^{30}$ The total cost difference can be significant where individual cost components are not because use of services in these cost categories is likely to be positively correlated (i.e. they add up in the same direction).
} 
Table 6-8 highlights a number of observations that are relevant. First, people in the sample with highvalue personal health budgets ("hi PHB" in the table) had much higher expenditure levels at baseline than people with low-value personal health budgets. Second, the growth in direct, indirect and total costs over the follow-up period was lower, both proportionally and in actual terms, for high-value personal health budget group compared to the low-value personal health budget group. Thirdly, compared to the change in costs for the control group (as reported in Table 6-7), participants with high-value personal health budgets showed lower growth in total ( $£ 3,100$ less growth) whilst those with low-value personal health budgets showed a very slightly higher growth $(+£ 170) .{ }^{31}$ The lower growth in total costs for high-value personal health budgets in the sample compared to the control group was sufficiently large for us to infer, with $90 \%$ confidence, that high-value personal health budgets reduce total costs relative to conventional service arrangements.

Table 6-8. Differences in service and support costs, by personal health budget type

\begin{tabular}{|c|c|c|c|c|c|c|c|c|}
\hline & \multicolumn{2}{|c|}{ Baseline cost totals } & \multicolumn{2}{|c|}{$\begin{array}{l}\text { Change in cost } \\
\text { between baseline } \\
\text { and follow-up }\end{array}$} & \multicolumn{2}{|c|}{$\begin{array}{l}\text { Difference-in- } \\
\text { difference } \\
\text { compared to the } \\
\text { control group }\end{array}$} & \multicolumn{2}{|c|}{ Significance of DiD } \\
\hline & Lo PHB & Hi PHB & Lo PHB & Hi PHB & Lo PHB & Hi PHB & Lo PHB & Hi PHB \\
\hline Social care & 5190 & 24530 & 3530 & 450 & 820 & -2270 & 0.464 & 0.103 \\
\hline Well-being & 0 & 140 & 390 & 680 & 390 & 690 & $<0.001^{* * *}$ & $<0.001^{* * *}$ \\
\hline Nursing \& therapy & 130 & 230 & 0 & 190 & 10 & 200 & 0.682 & $0.044^{* *}$ \\
\hline Other health & 0 & 4940 & 50 & 230 & -20 & 150 & $0.008^{* *}$ & $<0.001^{* * *}$ \\
\hline Sub-total: Direct costs & 5330 & 29830 & 3970 & 1550 & 1190 & -1220 & 0.237 & 0.349 \\
\hline Primary care & 560 & 870 & 20 & 120 & -50 & 50 & 0.362 & 0.470 \\
\hline Inpatient care & 3960 & 6990 & -1770 & -2740 & -940 & -1910 & $0.054^{*}$ & 0.132 \\
\hline Outpatient \& A\&E & 830 & 1090 & -140 & -110 & -40 & -20 & 0.326 & 0.812 \\
\hline Sub-total: Indirect costs & 5350 & 8950 & -1890 & -2730 & -1030 & -1880 & $0.044^{* *}$ & 0.144 \\
\hline Total cost & 10680 & 38790 & 2090 & -1180 & 170 & -3100 & 0.883 & $0.077^{*}$ \\
\hline
\end{tabular}

\subsubsection{Control factors}

As described above, there was no statistically significant difference in mean cost-change between personal health budget and control groups - see Table 6-3. We also tested the results to assess whether the difference became significant after introducing control factors. The multivariate estimations, using the same control factors as in chapter 5 , produced very similar results to the

\footnotetext{
${ }^{31}$ For low-value personal health budgets, direct costs in the sample showed greater growth on average compared to the control group.
} 
uncontrolled results in Table 6-3, with almost the same effect size (-f1125) and no significant difference $(p=0.26)$.

\subsection{Net monetary benefit}

Net (monetary) benefit (NMB) at baseline and follow-up was estimated for study participants in both the personal health budget and control groups. As outlined in section 6.3.4, personal health budgets would be assessed as cost-effective if they produced greater net benefit (i.e. $f$-value of benefits less cost) than conventional service arrangements (to a statistically significant degree). We made this assessment by comparing the net benefit at follow-up (less net benefit at baseline) in the personal health budget group with that of the control group. As we are controlling for baseline differences in the net benefit of support and services received by the two groups, we refer to net benefit change or 'NMB-change' and the difference between the groups in NMB-change.

The results are given in Table 6-9 for ASCOT and Table 6-10 for EQ-5D. Starting with the ASCOTmeasured benefits, Table 6-9 shows that over the follow-up period both personal health budget and control group participants showed an improvement in their ASCOT quality of life scores (as also described in chapter 5 ). The personal health budget group showed the greater improvement - by a difference of 0.039 . Seeing an improvement in the control group - albeit modest, of 0.018 on average - it is not unexpected because people in the control group were getting an increased level of services over the period due to an increase in need: the cost of their care increased by $f 1920$.

The table shows the $f$-value of this beneficial impact, by an amount that varies according to the willingness to pay threshold we use. For example, at the $£ 30,000$ threshold, the 0.057 improvement in ASCOT quality of life reported by the personal health budget group between follow-up and baseline is valued at $£ 1720$ (i.e. $£ 30,000 \times 0.057$ ). For the control group, benefits increased by $£ 540$ over the follow-up period. Overall, the personal health budget group were showing between $£ 400$ and $£ 1570$ worth of extra benefit over the control group, depending on the threshold chosen.

As outlined above, the costs of services and support for people in the personal health budget group increased at a slower rate on average than for the control group. Effectively, total service costs were f1120 less, on average, in the personal health budget group than the control group.

Taking both cost and benefits together, therefore, the personal health budget group were showing greater benefit and less cost, on average, than the control group, so net benefit was between $£ 1520$ and $£ 2690$ greater for the personal health budget group compared to the control group after subtracting baseline differences. For example, at the $f 30,000$ threshold, the extra net benefit averaged $f 2300$ ( $f 1180$ minus $-f 1120$ ) more for the personal health budget group compared to the control group.

Exactly the same calculations were undertaken for EQ5D-measured benefits - see Table 6-10. In this case, personal health budgets showed greater net benefit than controls on average of between $£ 1020$ and $£ 700$, though these were not statistically significant. 
Table 6-9. Difference in mean NMB-change for ASCOT, whole sample, various CE thresholds

\begin{tabular}{|c|c|c|c|c|c|c|}
\hline & PHB & Control & Difference & $\begin{array}{l}\text { Sig prob } \\
(p: \neq 0)\end{array}$ & $90 \% \mathrm{Cl}-$ & $90 \% \mathrm{Cl}+$ \\
\hline \multicolumn{7}{|l|}{ Benefits } \\
\hline ASCOT change & 0.057 & 0.018 & 0.039 & & & \\
\hline \multicolumn{7}{|c|}{ f-value of ASCOT change: } \\
\hline$£ 40,000$ & 2290 & 720 & 1570 & & & \\
\hline$£ 30,000$ & 1720 & 540 & 1180 & & & \\
\hline$£ 20,000$ & 1150 & 360 & 790 & & & \\
\hline$£ 10,000$ & 580 & 180 & 400 & & & \\
\hline \multicolumn{7}{|l|}{ Costs } \\
\hline Cost change & 800 & 1920 & -1120 & & & \\
\hline \multicolumn{7}{|l|}{ Net benefit } \\
\hline \multicolumn{7}{|l|}{ NMB change: } \\
\hline$£ 40,000$ & 1490 & -1200 & 2690 & $0.057^{*}$ & 410 & 4970 \\
\hline$£ 30,000$ & 920 & -1380 & 2300 & $0.082^{*}$ & 140 & 4460 \\
\hline$£ 20,000$ & 350 & -1560 & 1910 & 0.124 & -150 & 3960 \\
\hline$f 10,000$ & -220 & -1740 & 1520 & 0.198 & -450 & 3490 \\
\hline
\end{tabular}

Significance levels: ${ }^{*} p<0.10^{* *} p<0.05^{* * *} p<0.001$

Table 6-10. Difference in mean NMB-change for EQ-5D, whole sample, various CE thresholds

\begin{tabular}{|c|c|c|c|c|c|c|}
\hline & PHB & Control & Difference & $\begin{array}{l}\text { Sig prob } \\
(p: \neq 0)\end{array}$ & $90 \% \mathrm{Cl}-$ & $90 \% \mathrm{Cl}+$ \\
\hline \multicolumn{7}{|l|}{ Benefits } \\
\hline EQ-5D change & -0.011 & 0.000 & -0.011 & & & \\
\hline \multicolumn{7}{|c|}{$f$-value of EQ-5D change: } \\
\hline$£ 40,000$ & -420 & 0 & -420 & & & \\
\hline$£ 30,000$ & -310 & 0 & -310 & & & \\
\hline$£ 20,000$ & -210 & 0 & -210 & & & \\
\hline$£ 10,000$ & -100 & 0 & -100 & & & \\
\hline \multicolumn{7}{|l|}{ Costs } \\
\hline Cost change & 800 & 1920 & -1120 & & & \\
\hline \multicolumn{7}{|l|}{ Net benefit } \\
\hline \multicolumn{7}{|l|}{ NMB change: } \\
\hline$£ 40,000$ & -1220 & -1920 & 700 & 0.613 & -1710 & 3110 \\
\hline$£ 30,000$ & -1110 & -1920 & 810 & 0.536 & -1450 & 3060 \\
\hline$£ 20,000$ & -1010 & -1920 & 910 & 0.459 & -1200 & 3030 \\
\hline$£ 10,000$ & -900 & -1920 & 1020 & 0.386 & -980 & 3020 \\
\hline
\end{tabular}


As outlined above, although we found that the people in the personal health budget sample group did better than the people in the control group on average, this sample difference is only a noisy indicator of its true value. ${ }^{32}$ We therefore need to consider the statistical significance of the difference-in-difference estimator of NMB. Two methods are available to determine statistical significance: the conventional method is to assume that variation in results across all participants in the study follows a normal distribution - the parametric approach; an alternative is to use a repeated sampling method - the bootstrap approach - which does not make the same parametric assumptions about the shape of the distribution in the sample. These two methods produce the same results if the underlying distribution is normal. Using both methods helps us determine whether our results are influenced by the testing assumptions.

Our aim is to assess cost-effectiveness of personal health budgets compared to conventional service arrangements. The sample mean values of the difference in net benefit outlined above and the corresponding sample variance across participants in the study will reflect the true value but there is always a small chance that this sample gives a false result.

For ASCOT-measured benefits, we estimated that personal health budgets produced greater net benefit than conventional services with between a 0.198 and 0.057 probability of this being a false positive, depending on the threshold used - see Table 6-9. Taking the base $f 30,000$ threshold, we found that the rejection probability (of personal health budgets being cost-effective) was 0.082 . Another way of expressing this result is to say that personal health budgets are cost-effective with a better than $90 \%$ confidence level.

For EQ5D-measured benefits, the effect sizes were much smaller on average than for ASCOTmeasured benefits and therefore, accounting for statisical error, we could not reasonably reject the possibility that personal health budgets are not cost-effective when benefits are measured this way.

Figure 6-4 presents the significance results from the tables in graphical form - as confidence intervals around the point estimates.

\footnotetext{
${ }^{32}$ Even where it is an unbiased estimate of the true effect of using a personal health budget.
} 
Figure 6-4. ASCOT and EQ-5D NMB Diff-in-Diff, whole sample, point estimates and confidence intervals

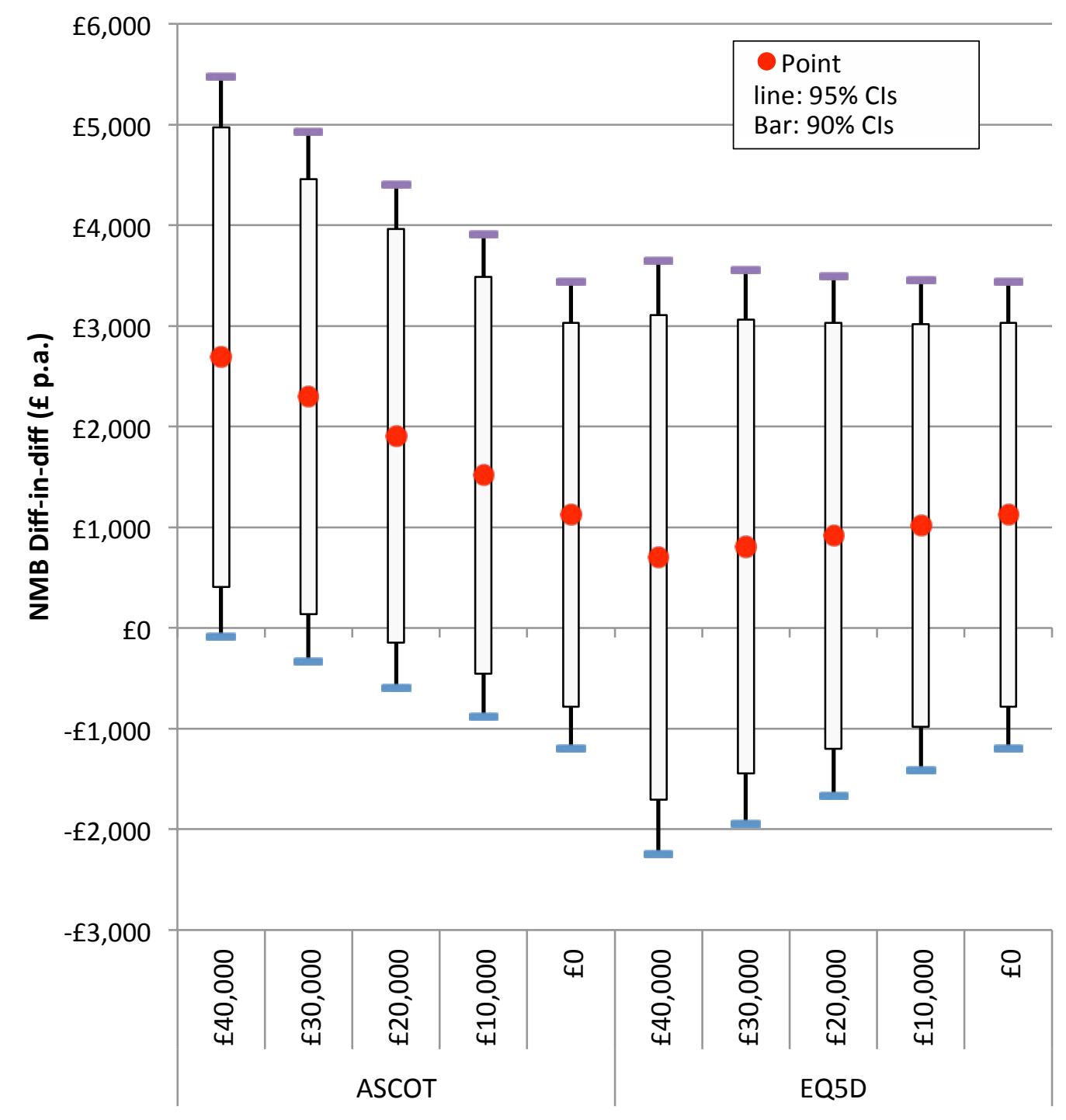

The significance results in the tables and the above chart are the 'parametric' versions. We also computed bootstrap significance results. These results were derived by creating 1000 re-samples from the original sample for each of the five imputations of the imputed dataset. In each case, we calculated cost-change and outcome-change difference, and also the difference in the NMB change. Rubin's rules were applied to adjust the estimated standard errors to allow for the imputed values in the data. ${ }^{33}$ Table 6-11 reports the results. Overall, they are very similar to the parametric results

\footnotetext{
${ }^{33}$ Because imputed missing values are estimates subject to statistical error, this additional source of statistical noise needs to be taken into account when calculating significance.
} 
about suggesting that the cost, outcome and $\mathrm{NMB}$ change variables are close to normally distributed. NMB using ASCOT outcomes is significant at the $10 \%$ level for the $£ 30,000$ threshold.

Table 6-11. Statistical significance using bootstrap results, ASCOT and EQ-5D, whole sample (£30, 000 threshold)

\begin{tabular}{lllllll}
\hline & & $\begin{array}{l}\text { Mean } \\
\text { effect }\end{array}$ & BS t stat & Prob & Cl- & Cl+ \\
\hline ASCOT & NMB-change & 2300 & 1.867 & $0.082^{*}$ & 135 & 4464 \\
& Outcome-change & 0.039 & 3.017 & $0.007^{* *}$ & 0.017 & 0.062 \\
& Cost-change & -1125 & 1.026 & 0.319 & -3033 & 784 \\
\hline \multirow{2}{*}{ EQ-5D } & NMB-change & 807 & 0.634 & 0.537 & -1449 & 3064 \\
& Outcome-change & -0.011 & 0.775 & 0.452 & -0.035 & 0.014 \\
\hline
\end{tabular}

Significance levels: ${ }^{*} p<0.10^{* *} p<0.05^{* * *} p<0.001$

\subsubsection{Scatter plots}

The significance results can be seen in the following cost-effectiveness scatter plots (Figure 6-5 and Figure 6-6). ${ }^{34}$ In addition to the mean effect sizes and significance summary statistics outlined above, these charts give a useful visual understanding of the strength of effect that was found.

The charts show each combination of outcome-change and cost-change from each bootstrap replication over five imputations. The cost-effectiveness charts are divided into four quadrants. Quadrant A - for outcome-cost pairs where outcomes (change) are higher for personal health budgets compared to control and cost (change) lower - it is the dominant quadrant. Quadrant $D$ is the dominated quadrant with estimates for which personal health budgets are both more costly and produce less quality of life ( $\mathrm{QoL})$ outcome compared to the controls.

Quadrants B and C involve a relative improvement in outcomes or costs, but not both, so our NMB sum has both a positive and negative component. In these two cases, we had to calculate the difference in NMB-change for each group. Plotted on the chart below are two rays passing through the origin. These lines are drawn through all points where NMB difference is zero. To the left of the ray, the difference in NMB (change) is positive - personal health budgets are cost-effective. To the right of the ray, the difference is negative - personal health budgets are not cost-effective. The angle of the ray depends on the cost-effectiveness threshold assumed. The solid ray corresponds to the $£ 30,000$ threshold, whilst the dashed line is the $£ 20,000$ threshold.

\footnotetext{
${ }^{34}$ These plots were manually adjusted for additional noise associated with imputed data using the between variation standard errors calculated for the bootstrap estimation. They should be taken as approximations to illustrate the overall difference-in-difference by outcome and cost. Not making this adjustment treats each data point as non-stochastic i.e. an actual observation which gives the impression of greater significance than the main results indicate.
} 
Figure 6-5 is the cost-effectiveness plot for the ASCOT outcome across the whole sample. The majority of points are in the dominant quadrant $A$ and the majority of the rest are also to the left of the zero-NMB line. Corresponding to the significance results above, we found that just under $95 \%$ of points were in the cost-effective region for personal health budgets. From the in-depth qualitative interviews, it was clear that organisational representatives thought that personal health budgets would have an impact on both costs and well-being:

"One case where [participant] needed a communication device, the standard communication devices provided by the NHS weighs about three kilos, very cumbersome, it's a bit like an old fashioned typewriter. She's lying in bed, she wouldn't be able to actually use this thing 'cause she couldn't hold it, but they also cost between five and seven thousand a time. We bought an iPad for E355, with that she can actually hold it, type on it as a keyboard, which is great for her communication, she can also use a voice synthesiser so she can verbalise but she can also, as a side effect, contact her peers via social network sites and help combat that feeling of being socially isolated" (Project Lead).

Figure 6-5. Cost-effectiveness plot - ASCOT outcome, whole sample

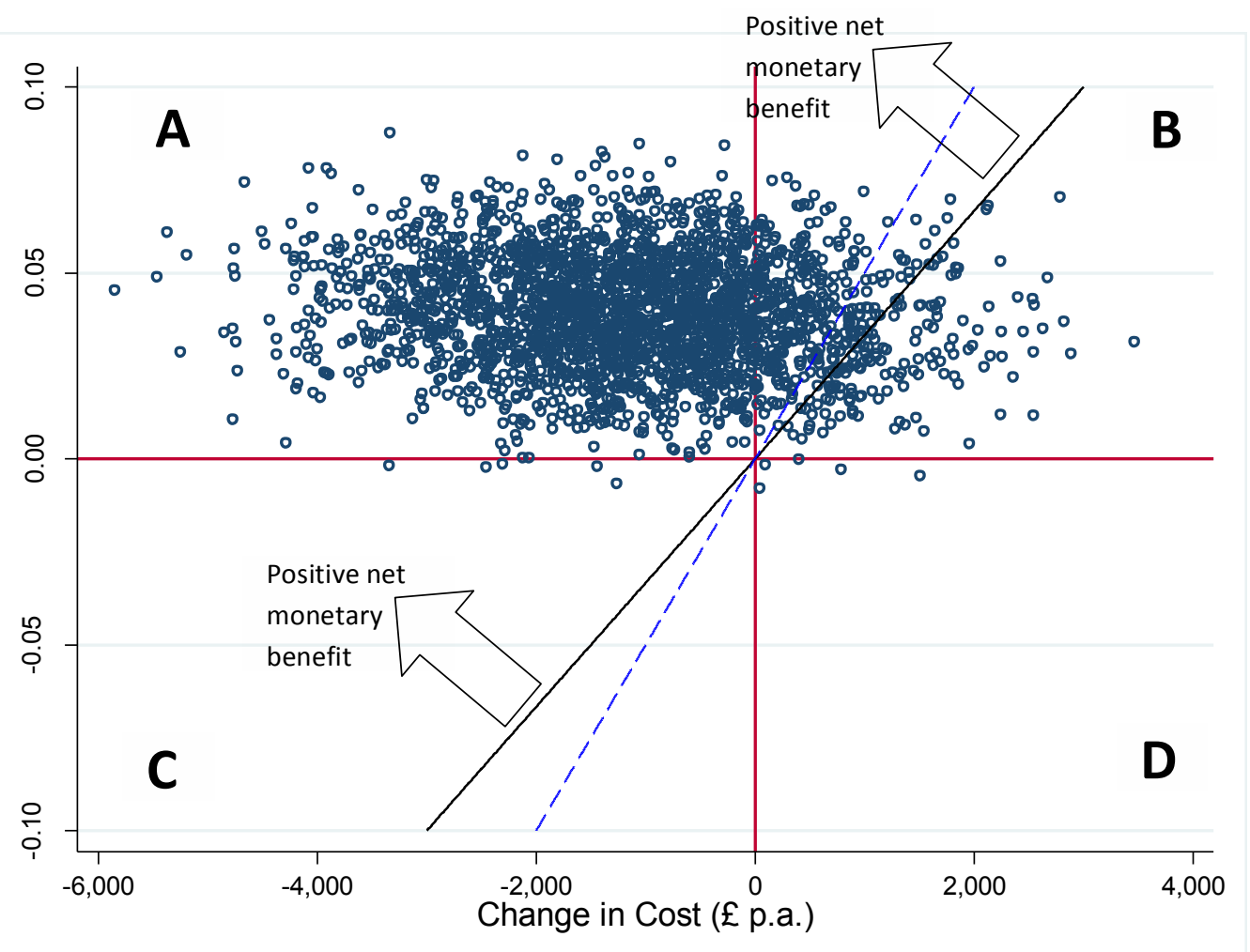

Regarding the EQ-5D plot (Figure 6-6), although the majority of points are in the cost-effective region, a significant number of points are in the non-cost-effective region (to the right of the zeroNMB line). Moreover, where points are in the cost-effective region this is due to personal health budgets having lower costs (change) than the control group. 
Figure 6-6. Cost-effectiveness plot - EQ-5D outcome, whole sample

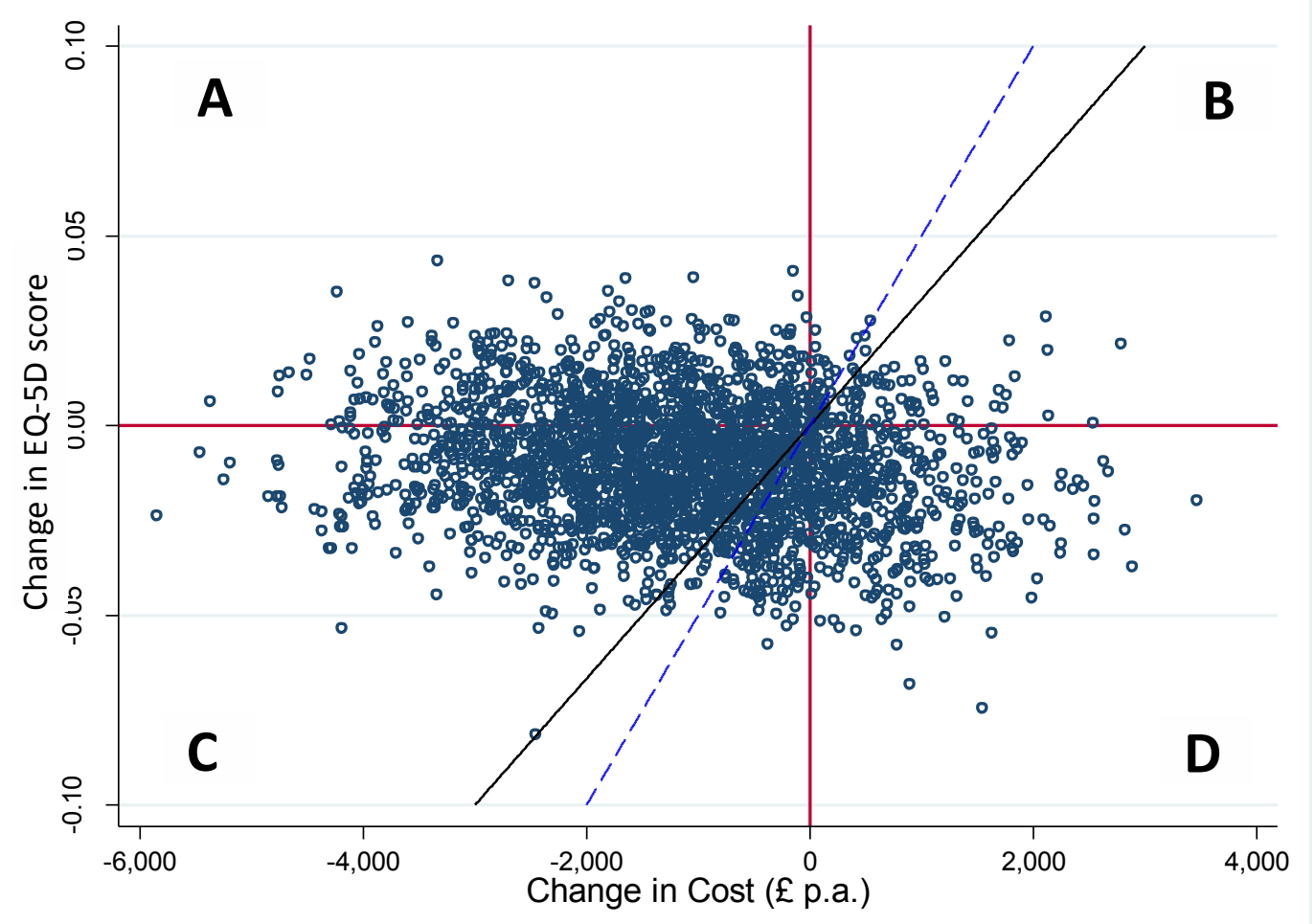

\subsubsection{Sub-groups}

Cost-effectiveness can be assessed for particular sub-groups within the sample to give us insight into which groups of participants did well, or not so well, with personal health budgets. We assessed the differential impact of personal health budgets by: participants' health condition; age group (e.g. people aged over 75); and the characteristics of the personal health budget model used in each case. In addition, we assessed the relative impact of personal health budgets by other socio-economic conditions, namely sex and income. We were unable to robustly investigate whether people from different ethnic groups have a different experience with personal health budgets because only $7.2 \%$ of the active sample where non-white. ${ }^{35}$

We also explored whether there were any differences between participants in the randomised subsample compared to the non-randomised sample in terms of the cost-effectiveness of personal health budgets.

\footnotetext{
${ }^{35}$ Bearing in mind the sample size issue, the analysis did not show a significant difference of effect by ethnic group (white compared to non-white).
} 
Sub-group effects were estimated using the difference between NMB-change for the people with personal health budgets in the sub-group and the people acting as controls in the same sub-group. This approach ensured that like-with-like comparisons of experience of personal health budgets or the usual care process were made given that there were differences between the sub-groups at baseline which needed to be removed. For example, people in the NHS Continuing Healthcare cohort were more intensive service users at baseline than the other cohorts. We therefore compared personal health budget holders in the NHS Continuing Healthcare cohort with controls in the NHS Continuing Healthcare cohort to assess cost-effectiveness for this sub-sample. When assessing the implications of particular implementation models for personal health budgets, we used the whole control group sample as the comparator because, by definition, they could not be differentiated by type of personal health budget (i.e. they had no personal health budget).

All of the sub-group analyses were for the $£ 30,000$ willingness-to-pay threshold. As indicated by Figure 6-4, these results would have to be adjusted up or down for difference thresholds.

\subsubsection{Health conditions}

Table 6-12 reports the sample mean difference between the personal health budget and control groups in changes in NMB for the six health conditions in the study. The analysis in the table takes the sub-sample of people in the study with the listed health condition and compares the change in NMB for that sub-sample with a personal health budget against people in the sub-sample with conventional service delivery. Because sample sizes in the health condition sub-groups are much smaller than for the whole sample, confidence intervals will be wider (or significance probabilities lower), other things equal. Finding a significant result in a given health condition sub-group means that the personal health budget effect size will have had to have been greater in that case than the personal health budget effect size for the whole sample at the same significance level. An alternative way of assessing whether there was any difference in the personal health budget effect size by subgroup was to test whether the PHB effect size for the sub-group was significantly different from the whole-sample PHB effect size. We did not find any significant differences from this latter analysis.

Table 6-12 reports the corresponding probabilities that this difference was significantly different from zero. For example, for the mental health cohort in the sample, average net benefit was $£ 4880$ greater for people in the personal health budget group compared to people in the control group (accounting for baseline differences). Figure 6-7 shows the confidence intervals for these estimates at both the $90 \%$ and $95 \%$ confidence levels. 
Table 6-12. NMB Diff-in-Diff, by health condition, point estimate and significance probability

\begin{tabular}{lrrrr}
\hline & \multicolumn{3}{c}{ ASCOT } & \multicolumn{3}{c}{ EQ-5D } \\
\hline & Coeff & prob & \multicolumn{1}{l}{ Coeff } & \multicolumn{1}{c}{ prob } \\
CHC & 9840 & $0.096^{*}$ & 6550 & 0.301 \\
Stroke & 3160 & 0.254 & 3320 & 0.227 \\
Diabetes & 510 & 0.768 & -670 & 0.708 \\
Mental health & 4880 & $0.096^{*}$ & 1810 & 0.489 \\
COPD & 1410 & 0.456 & 370 & 0.847 \\
\hline Neurological & -150 & 0.931 & -720 & 0.696 \\
\hline
\end{tabular}

Significance levels: ${ }^{*} p<0.10^{* *} p<0.05^{* * *} p<0.001$

Using the ASCOT scale, the results indicated that personal health budgets were cost-effective for both NHS Continuing Healthcare and mental health cohorts at the $10 \%$ significance level (or $90 \%$ confidence level). Otherwise we did not find significant differences between the groups. The final sample size for the NHS Continuing Healthcare sub-group was relatively small. ${ }^{36}$ Death rates in the NHS Continuing Healthcare cohort were also substantially higher than those for other groups. This meant that the NHS Continuing Healthcare results were subject to a high degree of noise which makes for wide confidence intervals. We also calculated bootstrap significance levels for this group which, given the small sample sizes, is especially important. The results, however, were essentially unchanged: bootstrap significance for NHS Continuing Healthcare was 0.097 for ASCOT and 0.302 for EQ-5D. Figure 6-8 and Figure 6-9 present indicative cost-effectiveness plots for the NHS Continuing Healthcare cohort.

The sample mean NMB difference-in-differences was high for the NHS Continuing Healthcare cohort. Where this is representative of the population of patients receiving NHS Continuing Healthcare, we would expect this result to be significant at higher levels with a larger sample size.

The mental health result is in keeping with the findings of the national evaluation of personal budgets in social care. This group appears to respond particularly well to personal health budgets.

\footnotetext{
${ }^{36}$ A number of participants within the NHS Continuing Healthcare cohort had moved into residential care by follow-up and, as a consequence, had to be excluded from the analysis.
} 
Figure 6-7. NMB Diff-in-Diff, by health condition, point estimates and confidence intervals

ASCOT

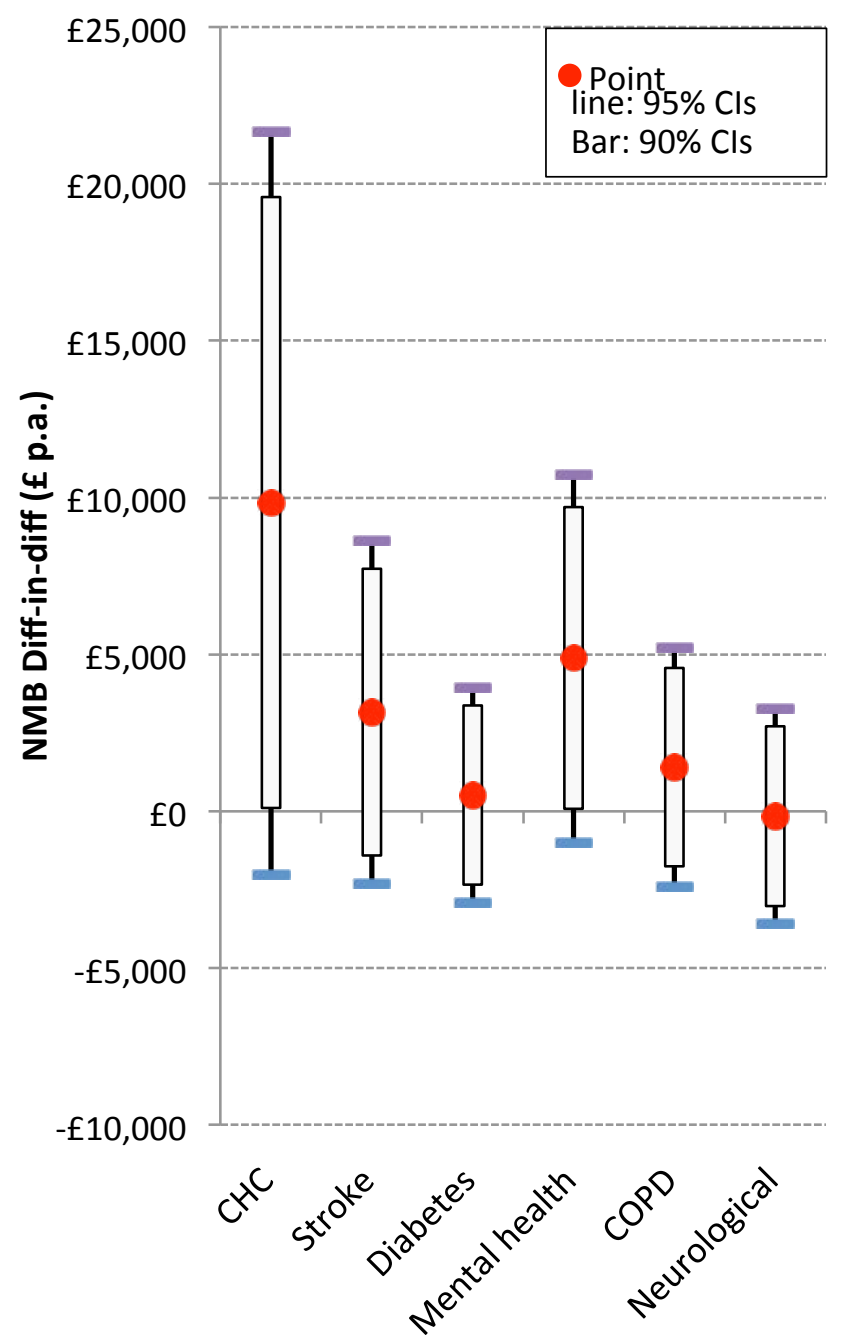

EQ-5D

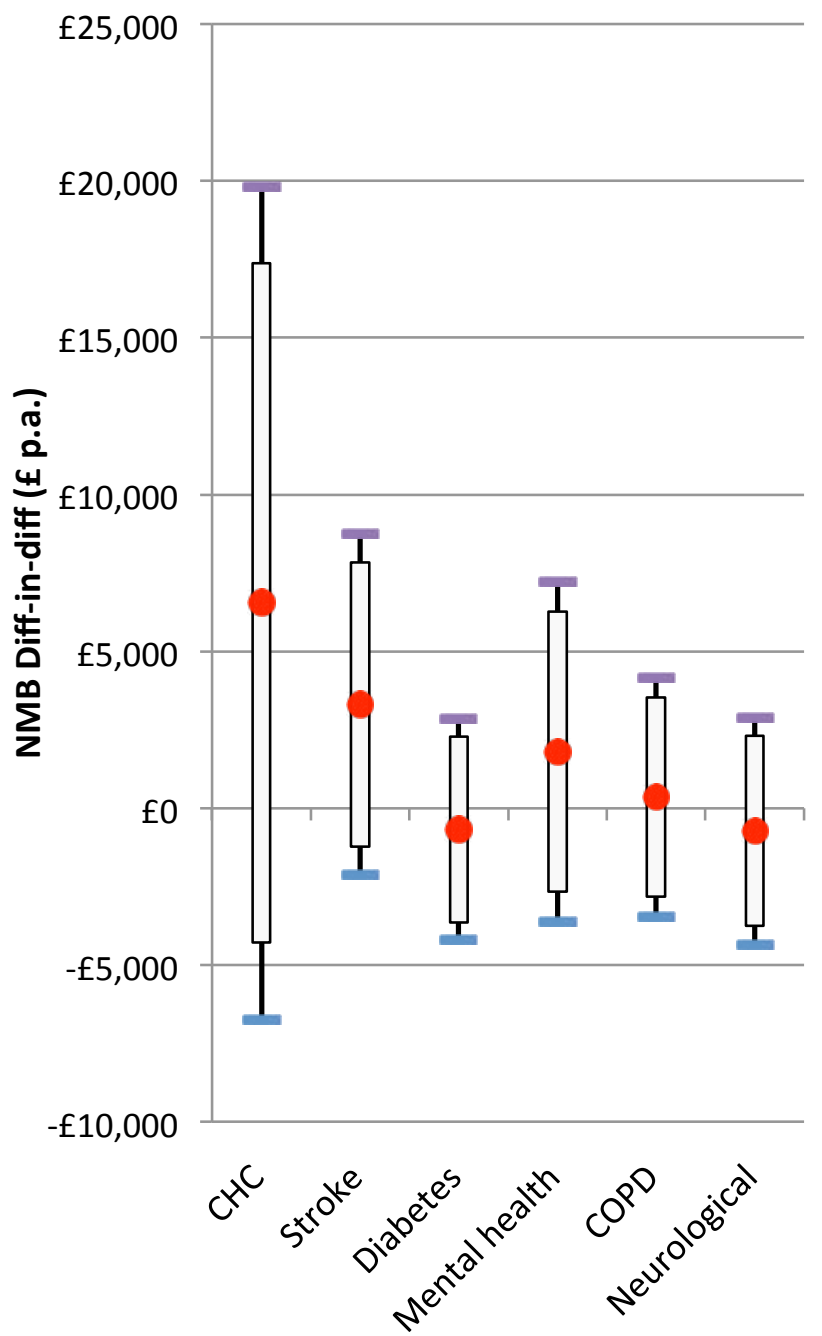

The bootstrap plots below show the different impacts of personal health budgets on ASCOT and EQ$5 \mathrm{D}$ outcomes. The personal health budget group in the sample reported better outcomes than the control group (from baseline) on ASCOT, but there was no significant difference on EQ-5D. By chance we might have a sub-sample of patients receiving NHS Continuing Healthcare in the personal health budget that were going to have poor EQ-5D outcomes anyway, regardless of their use of personal health budgets. We cannot reject this possibility at usual confidence levels for the EQ-5D indicator.

EQ-5D measures personal functioning and impairment in achieving good quality of life experiences whereas ASCOT measures achieved quality of life experiences, regardless of whether this achievement was via personal functioning or the support of services or others. This distinction is particularly sharp for the NHS Continuing Healthcare cohort where many people have high levels of personal impairment and accessed the services and support aimed at helping them to manage the consequences. In this regard we might infer that personal health budgets help people manage the consequences of their long-term conditions rather than improving their personal functioning. 
Figure 6-8. Cost-effectiveness plot - ASCOT outcome, NHS Continuing Healthcare sub-sample

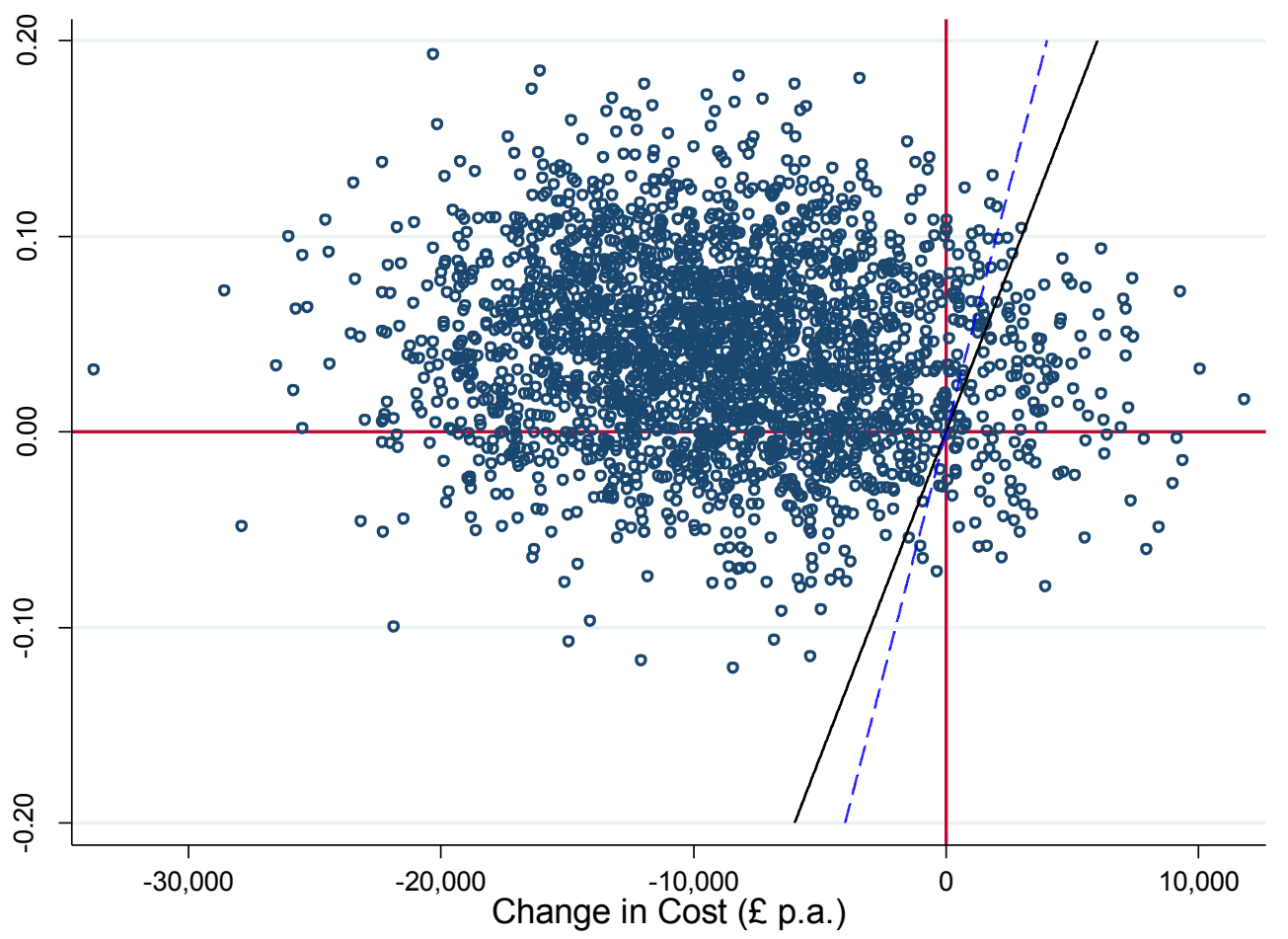

Figure 6-9. Cost-effectiveness plot - EQ-5D outcome, NHS Continuing Healthcare sub-sample

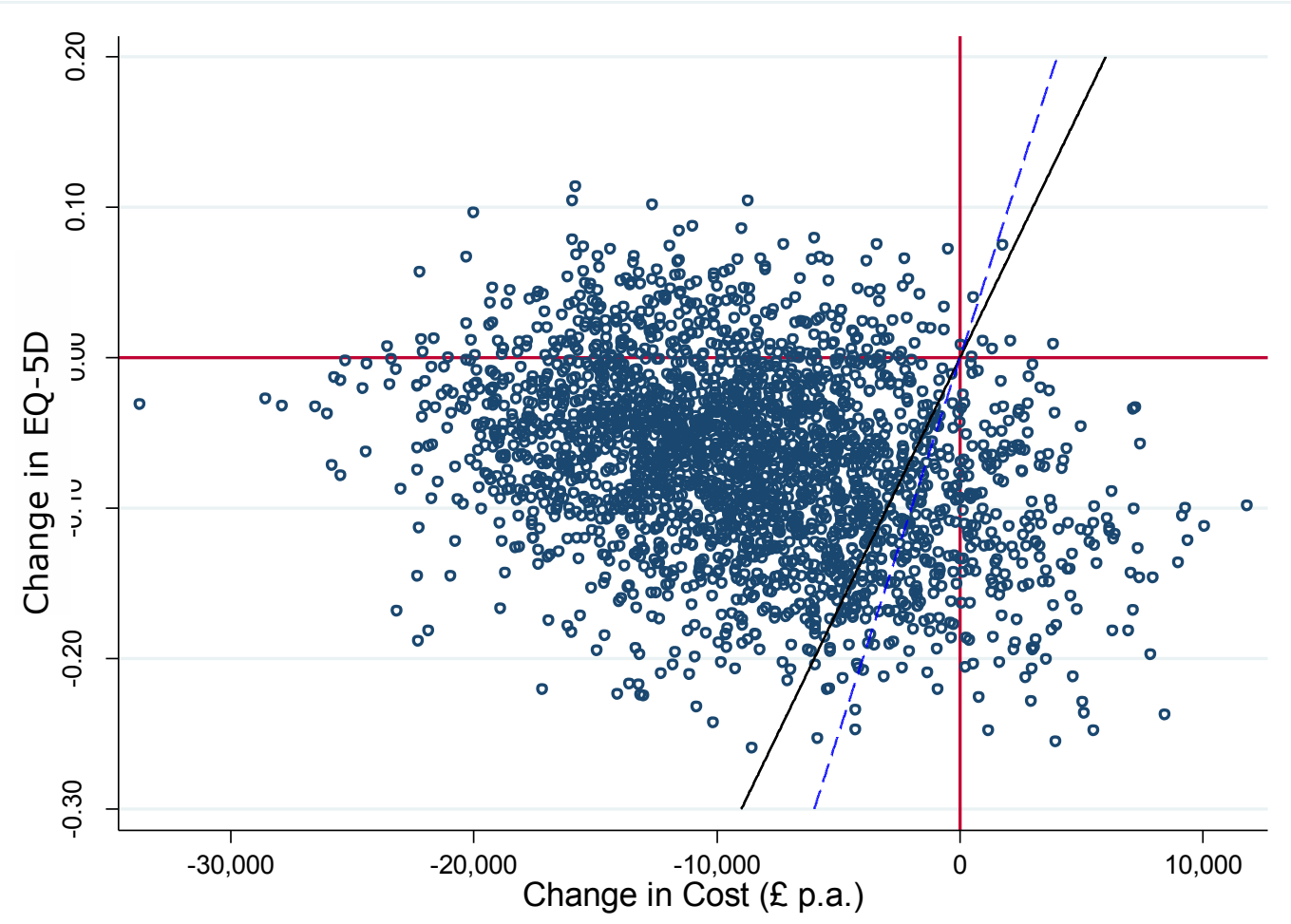




\subsubsection{Age group}

Regarding people aged over 75, those in the personal health budget group showed higher ASCOTmeasured net benefit than those in the control group (after subtracting for baseline differences). This effect was significant at the $90 \%$ confidence level $(p=0.055)$. The mean effect size was $£ 4610$ in the sample, with much of the additional net benefit coming from the cost savings (which were $f 4300$ less for the personal health budget group compared to the controls, after baseline, see Table 6-7) rather than improved quality of life benefits relative to the control group. This result does not necessarily mean that people over 75 gained no quality of life benefits from personal health budgets. Indeed, in that these personal health budget-holders were getting some $f 4300$ less support than their counterparts in the control group, which on its own could produce worst outcomes, we could argue that the direct benefits from personal health budgets offset these resource deficits - see Figure 6-1. We are able to explore this result using structural modelling - see section 6.6 below.

For people aged under 50 there was no significant difference between change in net benefit between the personal health budget and control groups (on the ASCOT scale). There were no significant differences between the groups on the EQ-5D scale for any age group - see Figure 6-10 and Table 6-13.

Table 6-13. NMB Diff-in-Diff, by age group, point estimate and significance probability

\begin{tabular}{llrc}
\hline & & \multicolumn{1}{c}{ Coeff } & \multicolumn{1}{c}{ Prob } \\
\hline ASCOT & Over 75 & 4610 & $0.055^{*}$ \\
& Under 50 & 2850 & 0.205 \\
\multirow{2}{*}{ EQ-5D } & Over 75 & 3870 & 0.128 \\
& Under 50 & 980 & 0.606 \\
\hline
\end{tabular}

Significance levels: ${ }^{*} p<0.10^{* *} p<0.05^{* * *} p<0.001$ 
Figure 6-10. NMB Diff-in-Diff, by age group, point estimates and confidence intervals

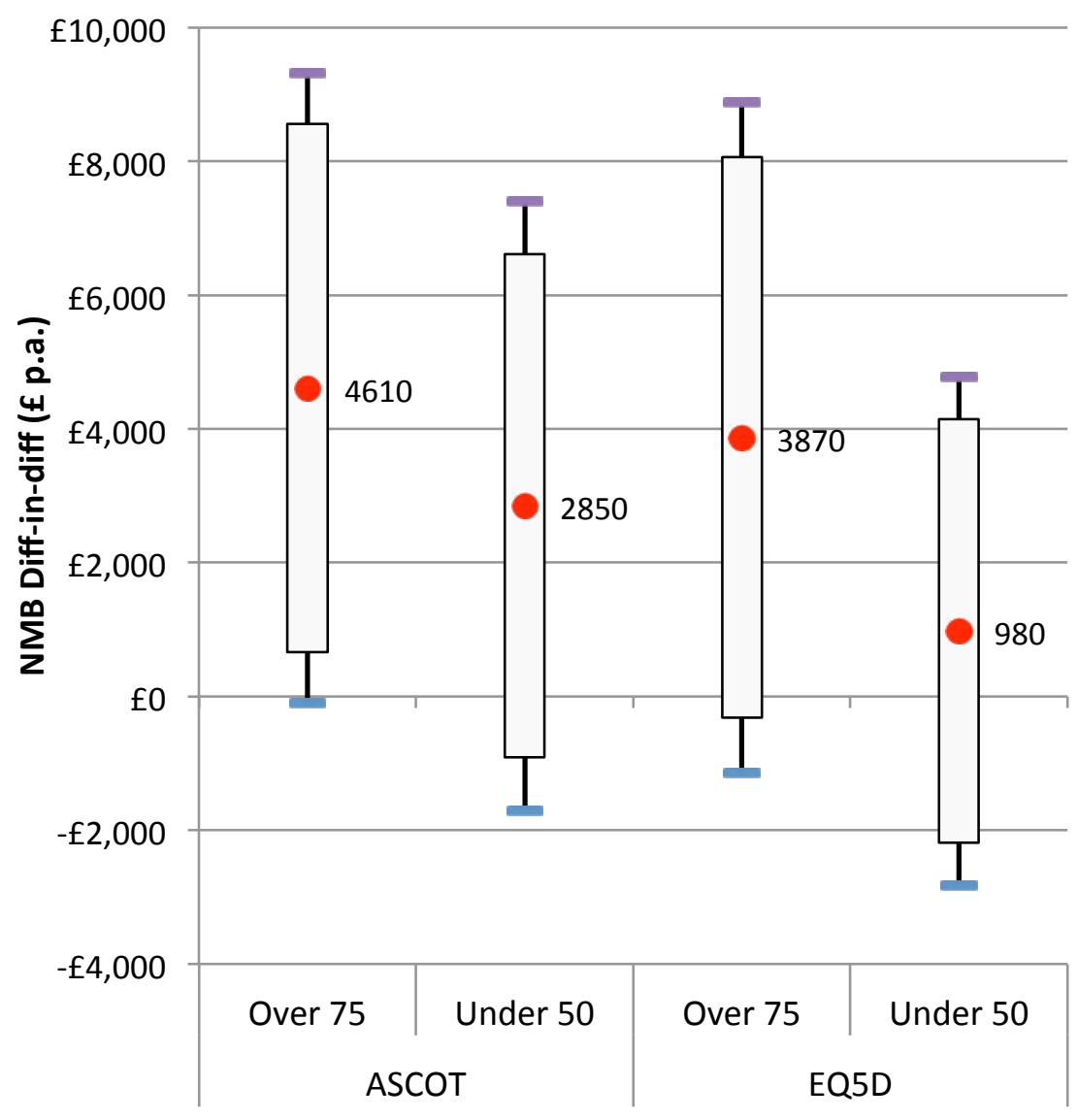

These results suggest that people aged over 75 have a greater capacity to benefit from personal health budgets than other age groups. Part of the reason for this result is that over $75 \mathrm{~s}$ have a greater use of services and so more opportunity to make savings with personal health budgets.

\subsubsection{Personal health budget process}

As outlined in Chapters 4 and 5, a range of implementation methods were used for personal health budgets, varying according to: the processes for determining the size of budgets; whether the budget is known before support planning; what flexibility there is in terms of what help can be purchased; the choice of deployment (including DPs); and so on. The range of specification options were reduced into five models for personal health budget implementation - see Table 1-3.

In Chapter 5 on outcomes we found models 1,4 and 5 to have significant positive impacts on ASCOT for personal health budgets compared to controls. As to EQ-5D, only model 3 showed significant results and these suggested that the personal health budget group had worsened with respect to the control group by follow-up. In this chapter we will focus on model 1 which can be regarded as the most ambitious form of personal health budget implementation, and model 3 which is perhaps furthest away from the policy intention, to assess the implications for cost-effectiveness in this 
section. ${ }^{37}$ We also looked at the implications of the size of budget and whether the budget is a direct payment for cost-effectiveness.

Table 6-14 shows the results. We found that people with high-amount personal health budgets (more than $\mathrm{f} 1000$ per year) were cost-effective compared to controls: on the ASCOT scale, highamount budgets were cost-effective at the $95 \%$ confidence level and on the EQ-5D scale, these personal health budgets were cost-effective at the $90 \%$ confidence level. In other words, whereas personal health budgets of all types were cost-effective with 90\% confidence levels using ASCOT, high-amount budgets were cost-effective at the higher $95 \%$ confidence level. This result is particularly apparent when looking at the cost-effectiveness plots for high-amount personal health budgets - see Figure 6-12 and Figure 6-13 below.

The results also indicated that personal health budgets were cost-effective at the $95 \%$ level for the ASCOT scale when implemented in Model 1 form. By contrast, personal health budgets in Model 3 form were not cost-effective compared to conventional practice in the control group.

Finally, personal health budgets were cost-effective (on the ASCOT scale) when deployed as direct payments as compared to the control group (at the $90 \%$ confidence level). For other deployments, effect sizes fell short of significant levels.

Table 6-14. NMB Diff-in-Diff, by personal health budget process, point estimate and significance probability

\begin{tabular}{llrl}
\hline & & Coeff & prob \\
\hline ASCOT & Hi Budget & 4340 & $0.025^{* *}$ \\
& PHB model 1 & 4830 & $0.041^{* *}$ \\
& PHB model 3 & 190 & 0.932 \\
& Direct payment & 3030 & $0.074^{*}$ \\
EQ5D & Hi Budget & 2850 & $0.099^{*}$ \\
& PHB model 1 & 2580 & 0.267 \\
& PHB model 3 & -1570 & 0.447 \\
& Direct payment & 1520 & 0.335 \\
\hline Significance levels: ${ }^{*} p<0.10^{* *} p<0.05^{* * *} p<0.001$
\end{tabular}

Confidence intervals for these three comparisons can be seen in Figure 6-11. As before the bootstrap, significance probabilities were very similar to the parametric versions. For high-value personal health budgets compared to controls, significance probabilities were 0.024 for ASCOT and 0.098 for EQ-5D. Regarding personal health budget model 1, the respective bootstrap significance probabilities were 0.041 for ASCOT and 0.266 for EQ-5D.

\footnotetext{
${ }^{37}$ We did test models 4 and 5 but these did not show significantly different results.
} 
Figure 6-11. NMB Diff-in-Diff, by personal health budget process, point estimates and confidence intervals

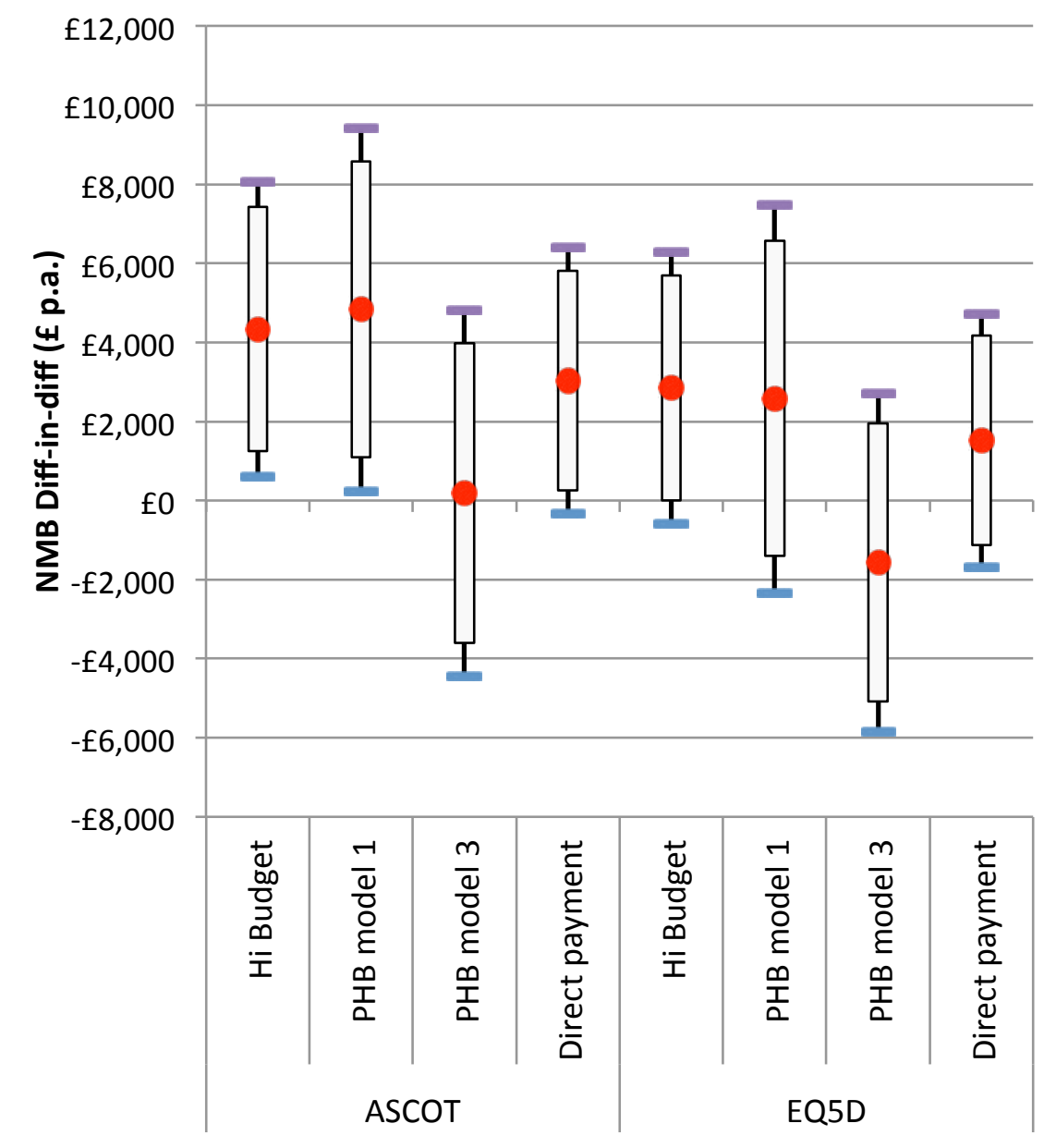


Figure 6-12. Cost-effectiveness plot - ASCOT outcome, personal health budget model 1

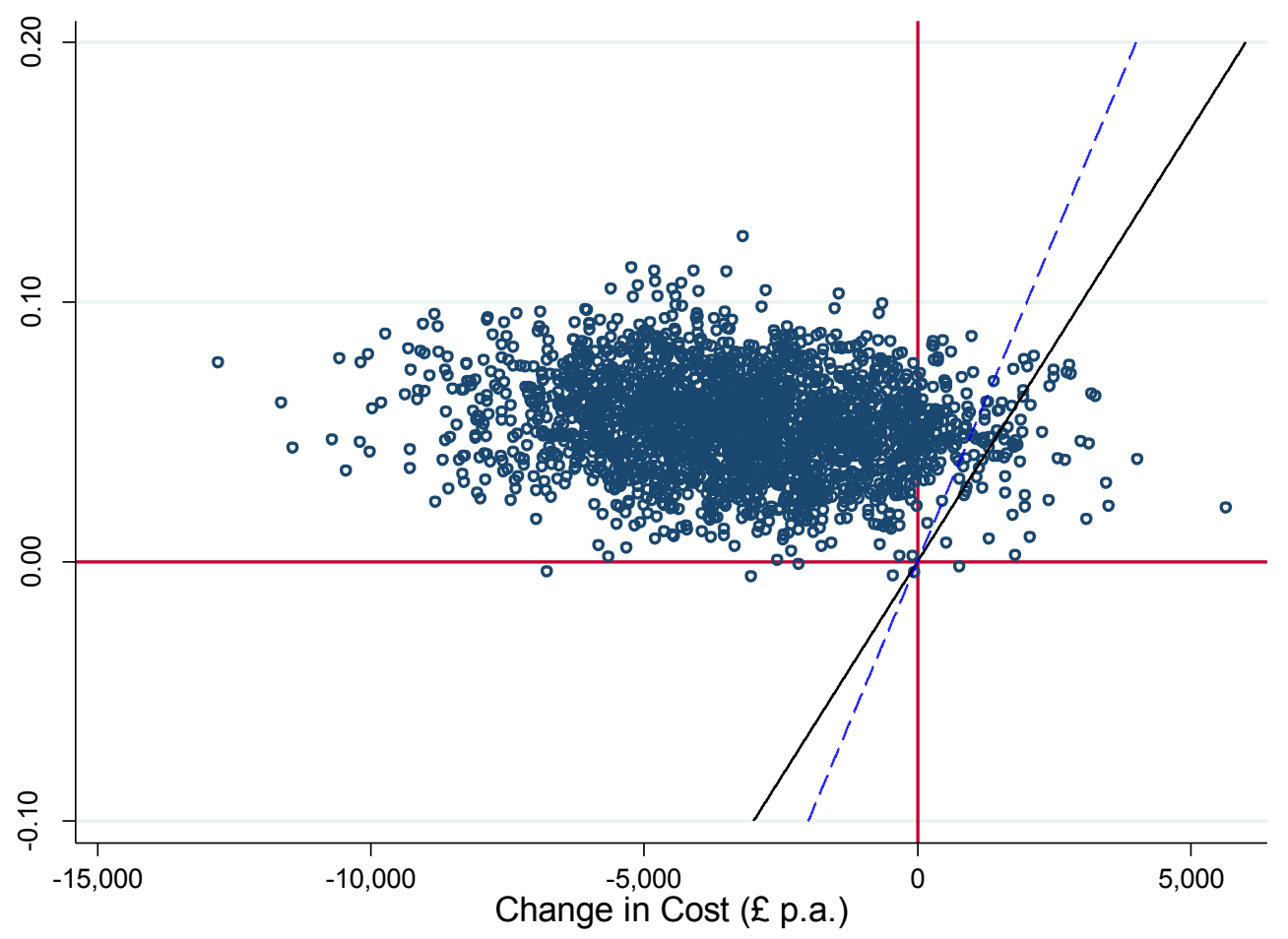

Figure 6-13. Cost-effectiveness plot - EQ-5D outcome, personal health budget model 1

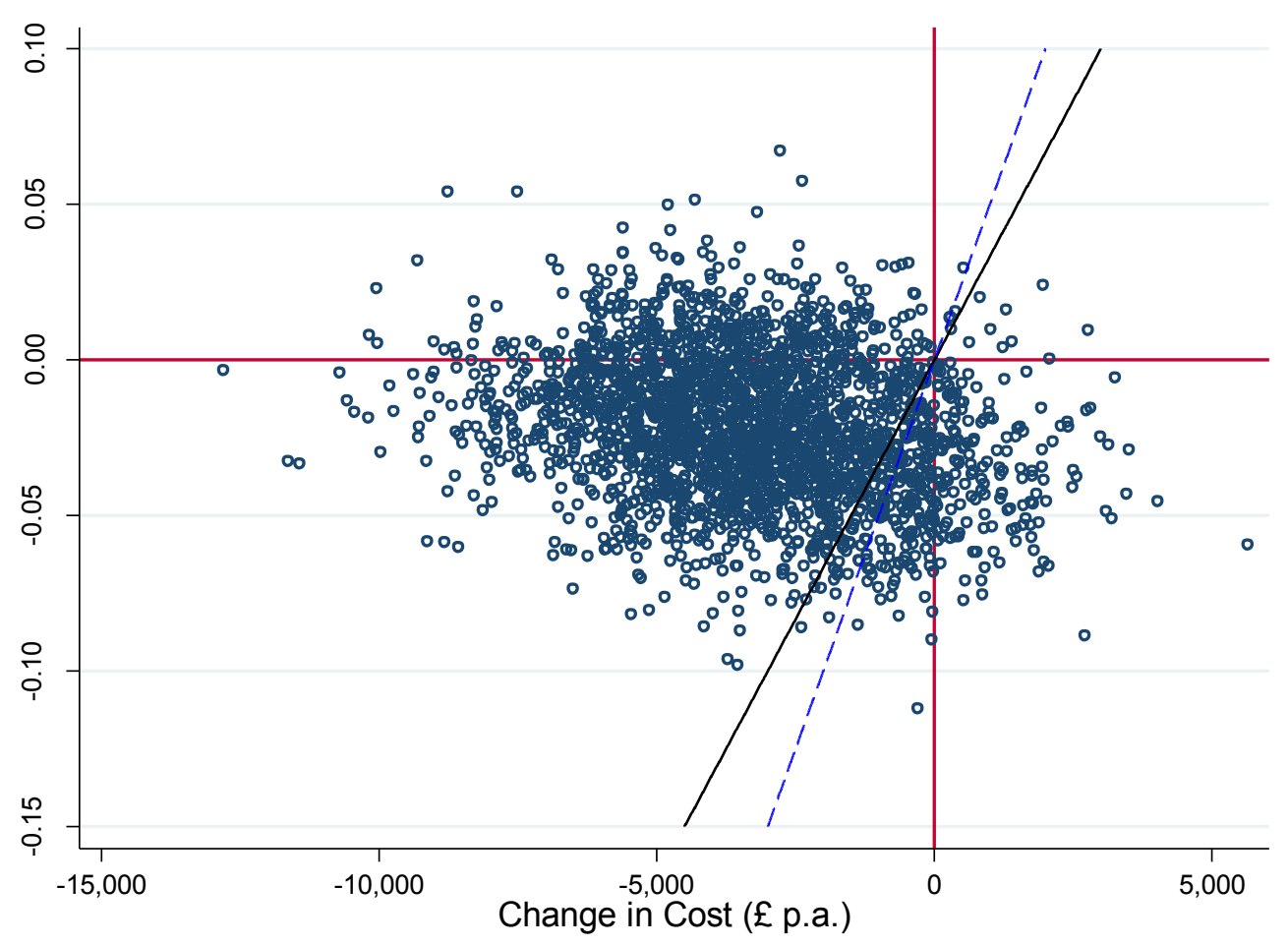




\subsubsection{Other socio-economic characteristics}

Further sub-group analyses were conducted by: gender (male); whether the participant reported claiming income benefits; and by reported university or college education. In none of these cases did we find a statistically significant differential effect of personal health budgets on net benefit. We surmise that the cost-effectiveness of personal health budgets was not affected by their sex, income status or education level. ${ }^{38}$

\subsubsection{Controlling for baseline characteristics on trend}

The difference-in-difference design of the study accounts for any differences between the personal health budget and control groups at baseline. It is possible, nonetheless, that differences between the groups at baseline affect how costs, outcomes and other variables change after baseline. If this pattern was characteristic of the data, we would expect differences between the groups at follow-up, after subtracting baseline values, even if personal health budgets had not been implemented and the 'personal health budget' group had continued with conventional support. As outlined in Appendix C, we can limit this potential problem by explicitly accounting for baseline characteristics that might cause differences in the rate of change of the variables of interest (i.e. cost and outcomes). For example, differences in age or baseline activities of daily living (ADL) disability might be relevant factors.

To this end, we gauged the impact of personal health budgets by estimating the NMB difference in difference between the personal health budget and control group after accounting for the variation in the change of NMB that was associated with a range of baseline factors (see equation C-9 in Appendix C). The factors are listed in Table 6-15 and include personal characteristics (e.g. age and sex), health conditions, characteristics of the areas in which the study participant lived, and some factors accounting for timings in the study. We estimated this difference-in-difference model for the whole sample and also for the sub-groups which showed significant cost-effectiveness above.

There is a risk in attempting to control for rate-of-change variation as we might inadvertently affect the impact of personal health budgets on cost-effectiveness. In particular, the control factors we used were significantly correlated with baseline service and support costs (e.g. someone with high ADL disability is more likely to have had higher service expenditure at baseline than someone with low need). We also know that the size of a person's personal health budget will be correlated with their baseline care needs (i.e. people with high service expenditure at baseline will have higher-value budgets, other things equal, than people with low baseline expenditure). As the above results suggest that the size of budget matters, we potentially introduce this bias when trying to control for extraneous change-of-change variation.

\footnotetext{
${ }^{38}$ Also, with the caution about small sub-sample sizes, no difference of effect was found by ethnic group.
} 
These deliberations suggest that using control factors might give further insight but that some caution is required in interpreting the results. ${ }^{39}$ Table 6-15 and Table 6-16 report our findings for the ASCOT NMB change. As regards to the base model (whole sample), the estimated mean increase in NMB change of the personal health budget group compared to the control group was $£ 1878$ rather than a mean difference of $£ 2300$ without additional control factors (see results in Table 6-9). The difference in these two results is well within the confidence intervals of the estimates and the (controlled) result remained significant at the $10 \%$ level.

We also tested the joint significance of the additional control factors together and found they were not significantly different from zero $(p=0.298)$. The same pattern of results occurred for the subgroup models. On the basis of these control factors, we can infer that there were not any significant differences in rate-of-change of NMB over and above the effect of personal health budgets.

We estimated similar models for EQ-5D. In all cases the additional control factors were (jointly) insignificant except for the NHS Continuing Healthcare sub-group, which was significant at the $10 \%$ level. The overall result of no cost-effective improvement from personal health budgets on EQ-5D outcomes was unaffected.

We tested the main sub-group results to see whether control factors made a difference. In all cases the control factors were jointly insignificant and had very small changes to the average effect sizes. This included sub-group analyses for personal health budget process, age and health condition. The only exceptions were for NHS Continuing Healthcare and diabetes using EQ-5D, where control factors were jointly significant, but where the difference in NMB change remained insignificant - see Table 6-12.

\footnotetext{
${ }^{39}$ These cautions would be especially relevant if we were to include baseline expenditure in the model as a control factor. We did try these estimations: baseline expenditure was highly significant with a co-efficient of near to one (as expected because baseline expenditure directly enters the difference-in-difference dependent variable on the left-hand side of the model). In the main this did not qualitatively affect the above results, with one exception. The interaction model with highand low- budget showed substantially changed coefficients.
} 
Table 6-15. ASCOT NMB Diff-in-Diff models with control factors - base, personal health budget size \& PHB model versions

\begin{tabular}{|c|c|c|c|c|c|c|}
\hline & \multicolumn{2}{|l|}{ Base } & \multicolumn{2}{|l|}{ PHB size } & \multicolumn{2}{|c|}{ PHB model 1} \\
\hline & Coeff & Prob & Coeff & Prob & Coeff & Prob \\
\hline PHB Group & 1877.75 & $0.089^{*}$ & & & & \\
\hline High-budget PHB & & & 3665.62 & $0.028^{* *}$ & & \\
\hline Low-budget PHB & & & 884.47 & 0.482 & & \\
\hline PHB, Model 1 & & & & & 4184.06 & $0.032^{* *}$ \\
\hline PHB, other model & & & & & 871.40 & 0.428 \\
\hline \multicolumn{7}{|l|}{ Person characteristics } \\
\hline Age & -242.05 & 0.306 & -217.33 & 0.347 & -237.98 & 0.316 \\
\hline Age (sqrd) & 1.76 & 0.411 & 1.54 & 0.462 & 1.75 & 0.417 \\
\hline Male & -106.30 & 0.927 & -90.61 & 0.938 & -27.31 & 0.981 \\
\hline ADL score & -297.89 & 0.372 & -258.56 & 0.439 & -306.32 & 0.360 \\
\hline ADL score (sqrd) & 5.65 & 0.548 & 5.52 & 0.559 & 6.12 & 0.515 \\
\hline Receives benefits & -2412.06 & $0.047^{* *}$ & -2446.35 & $0.042^{* *}$ & -2305.06 & $0.053^{*}$ \\
\hline \multicolumn{7}{|l|}{ Health condition cohort } \\
\hline $\mathrm{CHC}$ & 1019.17 & 0.716 & 638.52 & 0.823 & 163.71 & 0.951 \\
\hline Stroke & 2487.71 & 0.342 & 2793.68 & 0.287 & 3058.04 & 0.262 \\
\hline Diabetes & 1921.97 & 0.528 & 2102.49 & 0.498 & 2411.94 & 0.432 \\
\hline Mental health & 4560.93 & $0.068^{*}$ & 4676.93 & $0.062^{*}$ & 4497.07 & $0.073^{*}$ \\
\hline COPD & 47.87 & 0.987 & 267.78 & 0.928 & 239.02 & 0.936 \\
\hline Neurological & -204.67 & 0.941 & -87.99 & 0.975 & 15.26 & 0.996 \\
\hline \multicolumn{7}{|l|}{ Study timing } \\
\hline Follow-up period & -105.62 & 0.322 & -99.65 & 0.361 & -95.73 & 0.371 \\
\hline Follow-up period (sqrd) & 0.13 & 0.318 & 0.12 & 0.352 & 0.11 & 0.367 \\
\hline Consent date & -0.49 & 0.958 & -0.42 & 0.964 & -0.58 & 0.950 \\
\hline \multicolumn{7}{|l|}{ Area classification } \\
\hline ACA & 3721.43 & 0.697 & 3559.41 & 0.708 & 5070.13 & 0.600 \\
\hline Town \& Fringe location & 1895.77 & 0.385 & 1816.24 & 0.408 & 1542.58 & 0.484 \\
\hline Rural location & -247.20 & 0.919 & -166.24 & 0.946 & -713.62 & 0.775 \\
\hline \multicolumn{7}{|l|}{ Constant } \\
\hline Constant & 35677.58 & 0.839 & 31618.81 & 0.855 & 33502.60 & 0.847 \\
\hline Joint sig control factors & 1.17 & 0.289 & 1.08 & 0.367 & 1.08 & 0.367 \\
\hline RESET & 0.57 & 0.572 & 0.61 & 0.553 & -0.12 & 0.905 \\
\hline
\end{tabular}

Significance levels: ${ }^{*} p<0.10^{* *} p<0.05^{* * *} p<0.001$ 
Table 6-16. ASCOT NMB Diff-in-Diff models with control factors - base, personal health budget size \& PHB model versions

\begin{tabular}{|c|c|c|c|c|c|c|}
\hline & \multicolumn{2}{|l|}{ Over 75} & \multicolumn{2}{|l|}{$\mathrm{CHC}$} & \multicolumn{2}{|c|}{ Mental Health } \\
\hline & Coeff & Prob & Coeff & Prob & Coeff & Prob \\
\hline PHB Group, Over 75 & 4225.20 & $0.067^{*}$ & & & & \\
\hline PHB Group, Under 75 & 1360.81 & 0.243 & & & & \\
\hline PHB Group, $\mathrm{CHC}$ & & & 8958.35 & 0.10 & & \\
\hline PHB Group, Other health cohort & & & 1107.51 & 0.28 & & \\
\hline PHB Group, Mental health & & & & & 4152.71 & 0.120 \\
\hline PHB Group, Other health cohort & & & & & 1352.67 & 0.259 \\
\hline \multicolumn{7}{|l|}{ Person characteristics } \\
\hline Over 75 & -1370.57 & 0.623 & & & & \\
\hline Age & -252.61 & 0.438 & -224.15 & 0.347 & -221.54 & 0.339 \\
\hline Age (sqrd) & 1.84 & 0.570 & 1.65 & 0.444 & 1.59 & 0.450 \\
\hline Male & -84.60 & 0.941 & -81.30 & 0.944 & -110.92 & 0.923 \\
\hline ADL score & -294.93 & 0.377 & -312.12 & 0.350 & -306.32 & 0.358 \\
\hline ADL score (sqrd) & 5.58 & 0.553 & 6.29 & 0.502 & 5.77 & 0.540 \\
\hline Receives benefits & -2376.40 & $0.056^{*}$ & -2306.64 & $0.052^{*}$ & -2384.21 & $0.051^{* *}$ \\
\hline \multicolumn{7}{|l|}{ Health condition cohort } \\
\hline $\mathrm{CHC}$ & 1005.84 & 0.718 & -3774.48 & 0.231 & 1016.68 & 0.716 \\
\hline Stroke & 2480.21 & 0.341 & 2373.33 & 0.361 & 2371.10 & 0.368 \\
\hline Diabetes & 1907.60 & 0.529 & 1786.54 & 0.555 & 1772.79 & 0.568 \\
\hline Mental health & 4495.55 & $0.070^{*}$ & 4481.48 & $0.071^{*}$ & 2999.52 & 0.348 \\
\hline COPD & 104.98 & 0.972 & -47.57 & 0.987 & -23.31 & 0.994 \\
\hline Neurological & -208.29 & 0.940 & -322.17 & 0.907 & -297.18 & 0.915 \\
\hline \multicolumn{7}{|l|}{ Study timing } \\
\hline Follow-up period & -106.10 & 0.320 & -95.88 & 0.379 & -102.37 & 0.329 \\
\hline Follow-up period (sqrd) & 0.13 & 0.316 & 0.11 & 0.378 & 0.12 & 0.325 \\
\hline Consent date & -0.75 & 0.937 & -0.29 & 0.975 & 0.35 & 0.969 \\
\hline \multicolumn{7}{|l|}{ Area classification } \\
\hline ACA & 3960.895 & 0.680 & 3837.75 & 0.688 & 3697.75 & 0.699 \\
\hline Town \& Fringe location & 1982.995 & 0.366 & 1926.29 & 0.377 & 1846.70 & 0.396 \\
\hline Rural location & -192.693 & 0.937 & 70.85 & 0.976 & -405.20 & 0.867 \\
\hline \multicolumn{7}{|l|}{ Constant } \\
\hline Constant & 40841.43 & 0.818 & 29714.5 & 0.863 & 19124.79 & 0.911 \\
\hline Joint sig control factors & 1.14 & 0.313 & 1.12 & 0.334 & 0.82 & 0.666 \\
\hline RESET & 0.61 & 0.55 & -0.04 & 0.972 & 0.46 & 0.648 \\
\hline
\end{tabular}

\subsubsection{Randomisation}

The randomised sub-sample of the study involved people that had been offered the chance to participate in the study as a potential recipient of a personal health budget (or participating in the 
control group). In contrast, in the non-randomised sub-sample, people were either asked to participate as controls or they were offered a personal health budget. As a consequence, we might expect some differences between the controls in the randomised and non-randomised sub-samples as people in the former group were predisposed to the idea of having a personal health budget whilst those in the latter were not.

On this basis, we might anticipate a greater difference between the personal health budget and control group in the randomised sample as compared to those in the non-randomised sample, other things equal. On the other hand, although we adopted a difference-in-difference design to mitigate baseline selection between personal health budget and control groups, in the absence of an (albeit fully blind) randomisation process, some selection effects could have bled into the final result. The direction of any selection bias, should it have occurred, was difficult to predict however. The above analyses using the additional baseline control factors suggested that baseline selection bias was limited in the study.

We explored the hypothesis that the randomised sub-sample had a greater chance of showing personal health budgets to be cost-effective relative to usual practice in the control group by comparing the size and significance of the difference in the change in NMB for each sub-sample. We found that the randomised sub-sample showed a small change in NMB overall but that the difference in this change between personal health budget and controls was greater in the randomised subsample (mean difference in difference of $f 4140$ ) than the non-randomised sub-sample (mean difference in difference of $f 1710$ ). The difference between these two estimates was not statistically significantly different. We can infer from these results that randomisation did not play a statistically significant role in the overall results.

\subsection{Exploring the relationship between costs and benefits}

Figure 6-1 shows the three main hypothesised effect 'routes' for personal health budgets. It also shows that the costs and benefits are inter-related. In using the NMB approach, we are assessing the impact of personal health budgets in terms of the combined effect along the routes in the figure (and indeed along other routes not included in the figure). We are, in other words, considering the actual implementation of personal health budgets in the study, including choices about resourcing PHBs. In theory personal health budgets could be deployed without changes in resource levels: that is, where they are cost-neutral. Therefore, it would be useful to have estimates of the effect size for each of the three routes of effect. This set of estimates would allow us, for example, to get a better sense of the impact of personal health budgets without changes in resources.

One way to produce these individual effect sizes is to estimate a structural model using multivariate regression. Details of this method are given in Appendix $\mathrm{C}$. The challenge with estimating a structural model is the need to have all relevant factors included, which is very demanding in terms of data collection. In this study we had only sufficient data for a partial structural analysis, whereby we estimated the impact of personal health budgets on outcome change whilst removing the effect of service change and some condition-severity characteristics. Nonetheless, this analysis gave us an estimate of the direct choice and control effect of using personal health budgets as shown by effect 
route (1) in Figure 6-1 above. It also provided an estimate of the impact of a change services on quality of life (change).

The ASCOT results are presented in Table 6-17. This table shows three estimated (structural) equations for change in ASCOT between baseline and follow-up. In the base model, we estimated the impact of using a personal health budget for the whole PHB sample. The estimated effect of personal health budgets on ASCOT change, assuming no change in total expenditure, was 0.049 . This estimate corresponds to the 'choice and control' and 'changed utilisation/better tailoring' effects described in Figure 6-1 - effects (1) and (2). As a shorthand, we can described both effects together as the control/tailoring effect.

A change in total service expenditure was also found to have a significant positive effect on ASCOT change (with a coefficient of 0.012 for every $£ 1000$ difference in total cost). In other words, an increase in support from services produces an increase in ASCOT quality of life, other things equal. Similarly, improved ADL functioning was strongly associated with an increase in ASCOT score.

The effect size of 0.049 can be compared with the overall effect size of 0.039 reported in Chapter 5 (Table 5-7). This figure can be interpreted as the net impact of personal health budgets on ASCOT quality of life scores if personal health budgets have zero effect on total expenditure. In this case, we would expect this control/tailoring effect to be greater than the overall effect because the use of personal health budget was associated with a decrease in total expenditure (of on average $-\mathrm{f} 1120$ : see Table 6-3). ${ }^{40}$ Moreover, we found no significant effect of personal health budget on change in $A D L$ functioning or on use of informal care.

If we take the direct control/tailoring effect and add the service effect we get:

Net effect size $=0.049+0.012 / 1000 x-1120=0.036$

This net effect is very similar to the 0.039 value reported in Chapter 5 .

As reported above, there was some indication that personal health budgets were most effective for the NHS Continuing Healthcare and mental health sub-groups. On this basis, we estimated the effect of personal health budgets on these two sub-groups, again assuming no change in total expenditure (i.e. the control/tailoring effect). For the mental health sub-group, the control/tailoring effect was highly significant with a coefficient of 0.112 . As regards the NHS Continuing Healthcare cohort, the control/tailoring effect was estimated with a coefficient of 0.153 , although with a much wider confidence interval and significance at the $10 \%$ level. In both cases, these control/tailoring effects are much higher than their corresponding total effect as reported in Chapter 5 . This difference occurs for

\footnotetext{
${ }^{40}$ A Two Stage Least Squares model was used to estimate the results. In the first-stage equation, we found the impact of personal health budgets on total expenditure change to have a point estimate effect size of $-f 1210$ (rather than - $f 1120$ without controlling for other factors).
} 
both NHS Continuing Healthcare and mental health cohorts because the personal health budget group had much lower costs than the control group on average.

To sum up, the results suggest that the use of a personal health budget has a direct impact on quality of life via improved choice, control and tailoring of services to personal needs and circumstances. Personal health budgets also have an impact on expenditure/cost, both from a change in service need and also as a consequence of changed resourcing levels. A change in expenditure (i.e. due to a change in service use) also has an impact on quality of life, other things equal. So where personal health budgets lead to a change in service use/expenditure, they also have a knock-on effect on quality of life. This latter effect goes some way to offsetting the direct 'control/tailoring' effect. The EQ-5D measure is not especially sensitive to measuring the benefits of improved control and tailoring, particularly the value of choice and control. It is perhaps not surprising, therefore, that the service effects tend to dominate on EQ-5D leading to zero overall effect. ASCOT, by contrast, is designed for people with long-term conditions where choice and control in managing one's condition is highly valued.

We might conclude that personal health budgets would be more cost-effective if they were provided without a reduction in expenditure i.e. would have a greater net benefit improvement over the control group in that case. These analyses do suggest that, other things equal, giving people more support would improve their quality of life. But the use of personal health budgets in this study produced joint benefit and cost effects, and so we cannot assume that net benefits would be the size of the control/tailoring effect estimated above if the personal health budget were actually provided without a reduction in expenditure. We cannot be sure, for example, that personal health budgets would be cost-effective for high-value budgets without expenditure reductions when measured on the EQ-5D scale. Nonetheless, these results do suggest that the level of net benefit of personal health budgets compared to conventional arrangements - and hence whether we assess personal health budgets to be cost-effective - is sensitive to policy decisions about resourcing levels for budgets. We might, in other words, see a greater likelihood of personal health budgets being costeffective with higher levels of expenditure.

The analysis also gives us an indication of the marginal benefit of the extra spending on people in the study. For example, other things equal, the analysis suggests that an extra $£ 1000$ expenditure produces additional EQ-5D benefit of 0.016 on average (with a confidence interval of 0.0070 to 0.025). The point estimate translates to a marginal cost per (EQ-5D) QALY of $£ 62,500$. Even accounting for the uncertainty of the estimate there is a considerable likelihood that extra expenditure at this level is outside the cost-effective range. We might interpret this finding as a reason to scale back expenditure on this group, but this judgement is hard to make without a better understanding of the change in marginal benefit rates that result from lower levels of expenditure and also about alternative uses of this resource.

Table 6-18 has structural estimation results for the EQ-5D measure. The overall effect of personal health budgets on the change in EQ-5D was essentially zero - see Table 5-10. Controlling for change in total expenditure, the use of all personal health budgets (whole sample) showed a small positive, but insignificant coefficient. We also estimated the impact on EQ-5D change of high-value personal health budgets compared to controls. The overall effect from the uncontrolled analysis was again 
very close to zero -.008. Controlling for total expenditure change, however, produced a control/tailoring effect of 0.063 , which is significant at the $5 \%$ level. The high budgets group showed a change in total costs of some $£ 3100$ less than controls, which accounts for much of this result. On average, an increase in total expenditure of $£ 1000$ leads to a 0.016 increase in EQ-5D according to our results (at the point estimates), so $-f 3100$ would mean a change in EQ-5D of -0.05 , which largely offset the 0.063 direct effect.

Interaction effects for NHS Continuing Healthcare and mental health were also estimated for the EQ$5 \mathrm{D}$ change equation and, whilst the estimated control/tailoring effect sizes were much higher than overall effect for these two sub-groups respectively, neither was significantly different from zero. 
Table 6-17. Differences in ASCOT score - controlling for expenditure

\begin{tabular}{|c|c|c|c|c|c|c|}
\hline & \multicolumn{2}{|l|}{ Base } & \multicolumn{2}{|l|}{ CHC } & \multicolumn{2}{|c|}{ Mental health } \\
\hline & Co-eff & Prob & Co-eff & Prob & Co-eff & Prob \\
\hline \multicolumn{7}{|l|}{ Change in process } \\
\hline PHB group (all) & 0.049 & $0.008^{* *}$ & & & & \\
\hline $\mathrm{PHB}-$ not $\mathrm{CHC}$ & & & 0.039 & $0.039^{* *}$ & & \\
\hline $\mathrm{PHB}-\mathrm{CHC}$ & & & 0.153 & $0.072^{*}$ & & \\
\hline PHB - not $\mathrm{MH}$ & & & & & 0.033 & $0.041^{* *}$ \\
\hline $\mathrm{PHB}-\mathrm{MH}$ & & & & & 0.112 & $0.004^{* *}$ \\
\hline \multicolumn{7}{|l|}{ Other change characteristics } \\
\hline Total cost diff (predicted) (£1000s) & 0.012 & $0.004^{* *}$ & 0.012 & $0.004^{* *}$ & 0.012 & $0.005^{* *}$ \\
\hline Receives benefit (T1) & -0.030 & 0.223 & -0.028 & 0.244 & -0.028 & 0.255 \\
\hline Receives benefit (T2) & -0.003 & 0.907 & -0.005 & 0.848 & -0.003 & 0.897 \\
\hline Care from co-resident (T1) & -0.055 & $0.035^{* *}$ & -0.057 & $0.036^{* *}$ & -0.054 & $0.032^{* *}$ \\
\hline Care from co-resident (T2) & -0.017 & 0.662 & -0.015 & 0.693 & -0.015 & 0.686 \\
\hline Change in Care from others & 0.034 & $0.085^{*}$ & 0.035 & $0.085^{*}$ & 0.033 & $0.089^{*}$ \\
\hline Change in ADL need & 0.016 & $0.000^{* * *}$ & 0.016 & $0.000^{* * *}$ & 0.016 & $0.000^{* * *}$ \\
\hline Change in ADL need - $\mathrm{CHC}$ group & $-2.99 \mathrm{E}-04$ & 0.950 & $1.05 \mathrm{E}-03$ & 0.840 & $-8.78 \mathrm{E}-04$ & 0.853 \\
\hline \multicolumn{7}{|l|}{ Invariant characteristics } \\
\hline Age & -0.006 & -2.340 & -0.007 & -2.400 & -0.005 & -2.000 \\
\hline Age squared & 4.47E-05 & 1.890 & $4.63 \mathrm{E}-05$ & 1.930 & $3.72 \mathrm{E}-05$ & 1.580 \\
\hline Age, $\mathrm{CHC}$ cohort & $-2.75 \mathrm{E}-04$ & -0.220 & $3.41 \mathrm{E}-04$ & 0.310 & $-5.80 \mathrm{E}-04$ & -0.470 \\
\hline Married & 0.055 & 2.700 & 0.056 & 2.740 & 0.053 & 2.610 \\
\hline Male & 0.016 & 0.990 & 0.017 & 1.030 & 0.016 & 0.980 \\
\hline Married males & -0.042 & -1.880 & -0.043 & -1.900 & -0.042 & -1.880 \\
\hline $\mathrm{CHC}$ cohort & 0.177 & 1.400 & 0.110 & 1.010 & 0.187 & 1.490 \\
\hline Stroke cohort & 0.160 & 1.810 & 0.195 & 1.930 & 0.153 & 1.730 \\
\hline Diabetes cohort & 0.188 & 1.970 & 0.223 & 2.050 & 0.181 & 1.880 \\
\hline Mental health cohort & 0.144 & 1.600 & 0.178 & 1.760 & 0.100 & 1.050 \\
\hline COPD cohort & 0.168 & 1.910 & 0.203 & 1.960 & 0.164 & 1.860 \\
\hline Neurological cohort & 0.165 & 1.900 & 0.198 & 1.950 & 0.162 & 1.860 \\
\hline Area cost adjustment & 0.132 & 0.690 & 0.122 & 0.610 & 0.129 & 0.680 \\
\hline Pilot site dummies & Yes & & Yes & & Yes & \\
\hline Constant & -0.114 & 0.612 & -0.125 & 0.577 & -0.125 & 0.578 \\
\hline \multicolumn{7}{|l|}{ Model } \\
\hline F-test & 2.84 & $<0.0001^{* * *}$ & 2.71 & $<0.0001^{* * *}$ & 2.87 & $<0.0001^{* * *}$ \\
\hline \multicolumn{7}{|l|}{ Weak identification } \\
\hline $\mathrm{F}$ & 7.28 & 0.0014 & 6.75 & 0.0023 & 7.39 & 0.0013 \\
\hline \multicolumn{7}{|l|}{ Over-identification } \\
\hline Sargan Chi2 & 1.406 & 0.495 & 1.338 & 0.512 & 1.427 & 0.490 \\
\hline \multicolumn{7}{|l|}{ Endogeneity } \\
\hline Hausman (t-test) & -3.21 & 0.004 & -3.18 & 0.005 & -3.15 & 0.005 \\
\hline
\end{tabular}

Note 1: $\mathrm{CHC}$ refers to NHS Continuing Healthcare

Significance levels: ${ }^{*} p<0.10^{* *} p<0.05{ }^{* * *} p<0.001$ 
To sum up, the results suggest that the use of a personal health budget has a direct impact on quality of life via improved choice, control and tailoring of services to personal needs and circumstances. Personal health budgets also have an impact on expenditure/cost, both from a change in service need and also as a consequence of changed resourcing levels. A change in expenditure (i.e. due to a change in service use) also has an impact on quality of life, other things equal. So where personal health budgets lead to a change in service use/expenditure, they also have a knock-on effect on quality of life. This latter effect goes some way to offsetting the direct 'control/tailoring' effect. The EQ-5D measure is not especially sensitive to measuring the benefits of improved control and tailoring, particularly the value of choice and control. It is perhaps not surprising, therefore, that the service effects tend to dominate on EQ-5D leading to zero overall effect. ASCOT, by contrast, is designed for people with long-term conditions where choice and control in managing one's condition is highly valued.

We might conclude that personal health budgets would be more cost-effective if they were provided without a reduction in expenditure i.e. would have a greater net benefit improvement over the control group in that case. These analyses do suggest that, other things equal, giving people more support would improve their quality of life. But the use of personal health budgets in this study produced joint benefit and cost effects, and so we cannot assume that net benefits would be the size of the control/tailoring effect estimated above if the personal health budget were actually provided without a reduction in expenditure. We cannot be sure, for example, that personal health budgets would be cost-effective for high-value budgets without expenditure reductions when measured on the EQ-5D scale. Nonetheless, these results do suggest that the level of net benefit of personal health budgets compared to conventional arrangements - and hence whether we assess personal health budgets to be cost-effective - is sensitive to policy decisions about resourcing levels for budgets. We might, in other words, see a greater likelihood of personal health budgets being costeffective with higher levels of expenditure.

The analysis also gives us an indication of the marginal benefit of the extra spending on people in the study. For example, other things equal, the analysis suggests that an extra $£ 1000$ expenditure produces additional EQ-5D benefit of 0.016 on average (with a confidence interval of 0.0070 to 0.025). The point estimate translates to a marginal cost per (EQ-5D) QALY of $£ 62,500$. Even accounting for the uncertainty of the estimate there is a considerable likelihood that extra expenditure at this level is outside the cost-effective range. We might interpret this finding as a reason to scale back expenditure on this group, but this judgement is hard to make without a better understanding of the change in marginal benefit rates that result from lower levels of expenditure and also about alternative uses of this resource. 
Table 6-18. Differences in EQ-5D score - controlling for expenditure

\begin{tabular}{|c|c|c|c|c|}
\hline & Co-eff & Prob & Co-eff & Prob \\
\hline \multicolumn{5}{|l|}{ Change in process } \\
\hline PHB, hi budget & 0.063 & $0.038^{* *}$ & & \\
\hline PHB group, all budgets & & & 0.015 & 0.425 \\
\hline \multicolumn{5}{|l|}{ Other change characteristics } \\
\hline Change in Total cost (predicted) ( $£ 1000 s)$ & 0.016 & $0.002^{* *}$ & 0.016 & $0.002^{* *}$ \\
\hline Change in Care from co-resident & -0.007 & 0.811 & -0.008 & 0.784 \\
\hline Change in Care from others & 0.027 & 0.132 & 0.025 & 0.155 \\
\hline Change in ADL need & 0.024 & $0.000^{* * *}$ & 0.024 & $0.000^{* * *}$ \\
\hline Change in $\mathrm{ADL}$ need - $\mathrm{CHC}$ cohort & 0.006 & 0.133 & 0.006 & 0.162 \\
\hline \multicolumn{5}{|l|}{ Invariant characteristics } \\
\hline Age, log & -0.004 & 0.803 & -0.008 & 0.644 \\
\hline Uni education & 0.015 & 0.323 & 0.014 & 0.348 \\
\hline Married & 0.012 & 0.568 & 0.013 & 0.538 \\
\hline Male & 0.006 & 0.796 & 0.006 & 0.788 \\
\hline $\mathrm{CHC}$ cohort & 0.124 & $0.082^{*}$ & 0.144 & $0.061^{*}$ \\
\hline Stroke cohort & 0.164 & $0.020^{* *}$ & 0.165 & $0.019^{* *}$ \\
\hline Diabetes cohort & 0.147 & $0.082^{*}$ & 0.152 & $0.070^{*}$ \\
\hline Mental health cohort & 0.122 & 0.148 & 0.128 & 0.124 \\
\hline COPD cohort & 0.132 & $0.079^{*}$ & 0.134 & $0.071^{*}$ \\
\hline Neurological cohort & 0.128 & $0.064^{*}$ & 0.131 & $0.054^{*}$ \\
\hline Pilot site dummies & Yes & & Yes & \\
\hline Constant & -0.133 & 0.198 & -0.108 & 0.254 \\
\hline \multicolumn{5}{|l|}{ Model } \\
\hline F-test & 4.22 & $<0.001^{* * *}$ & 4.16 & $<0.001^{* * *}$ \\
\hline \multicolumn{5}{|l|}{ Weak identification } \\
\hline $\mathrm{F}$ & 6.17 & $0.004^{* *}$ & 6.12 & $0.004^{* *}$ \\
\hline \multicolumn{5}{|l|}{ Over-identification } \\
\hline Sargan Chi2 & 4.13 & 0.126 & 4.38 & 0.112 \\
\hline \multicolumn{5}{|l|}{ Endogeneity } \\
\hline Hausman (t-test) & -3.79 & 0.003 & -3.80 & 0.003 \\
\hline
\end{tabular}

\subsection{Sensitivity analysis}

A number of assumptions were made in the course of this analysis. They fall into two categories: those around the statistical modelling of missing data in the multiple imputation process; and others made during the costing of personal health budgets as compared to the control group. We explored the sensitivity of our main results to changes in these assumptions by re-estimating net benefit differences with changes in assumptions as follows:

- Using a different imputation dataset (created by added further imputations);

- Using a different imputation model; 
- Selecting different sub-sample of the data for imputation;

- Changing assumptions about which personal health budgets substitute for, rather than be provided in-addition to, conventional services.

Details of the results are in the Annex to this chapter. In summary, the sensitivity analysis supported the main findings outlined above for all but the most extreme assumptions. In many cases the costeffectiveness results were stronger, with personal health budgets showing cost-effectiveness (on the ASCOT scale) at the $95 \%$ confidence level.

\subsection{Discussion}

Personal health budgets were hypothesised to change costs and benefits for study participants in three ways:

- Personal health budgets could have a direct effect on quality of life by improving people's choice and control over their own lives;

- Personal health budgets could allow people to change their utilisation of services and support to better match their own needs and preferences (other things equal this would also improve their quality of life);

- the use of personal health budgets might imply different overall resourcing levels for each recipient, compared to their situation without a personal health budget, receiving conventional support.

The three effects would change the amount and mix of services and support people use and thus change costs compared to the control group. They also have consequences for care-related quality of life and so might change the benefits people gain from the services and support they use. These considerations framed our analysis of costs and benefits.

As a system level reform, the use of personal health budgets can affect the use of a wide range of services and support, and these can differ significantly from the services available under conventional practice. Ideally we would aim to measure the total use of all forms of service and support for all participants in the study, regardless of funding source, but this was clearly infeasible in any practical evaluation. Instead, we collected information about what services and support people purchased or secured with their budgets, as well service use in a broad range of conventional service categories from respite care to inpatient hospital services. People in the personal health budget group used a mix of both conventional services and those funded from their budgets. Moreover, the coverage, possible use, size and specification of personal health budgets implementation models varied significantly between sites and by health condition. There was also a very broad mix of participants in the pilot who varied significantly according to their service use.

Both the personal health budget and control groups showed increased total cost averages between baseline and follow-up, although the rate of increase was slightly lower for the personal health budget group. Overall, in the personal health budget group the average cost increase was $f 800$ per person per year as compared to a $£ 1920$ increase for the control group, a difference of $f 1120$ less for the personal health budget group. The variety of both personal health budget implementations and 
study participants meant there was a high degree of variation of each participant's cost around these sample mean values so that the differences were not statistically significant. In other words, although we found in this sample that people in the personal health budget group had lower costs than those in the control group overall, we could not be confident (at the $10 \%$ level or better) that the true cost effect of personal health budgets is actually different from zero.

Total costs are a sum of the cost of direct services (those which can be purchased using a personal health budget) and indirect services (those which cannot by secured using a personal health budget e.g. hospital care). Direct costs showed very little difference between personal health budget and control groups. However, indirect costs were significantly lower for people using personal health budgets. This latter effect was not strong enough to carry through to produce a significant difference in total costs, as noted.

Overall, we did not find significant differences in the change in total costs between the groups. Some differences were significant, however, for sub-groups. In the mental health cohort, individuals' indirect costs (mainly inpatient costs) were reduced by a significantly greater amount in the personal health budget group than in the control group (significant at 1\%). A similar result occurred for the NHS Continuing Healthcare sample, but at the $10 \%$ significance level.

People gain benefit from using services and support as arranged with personal health budgets or through conventional means. Personal health budgets might affect the size of the benefits by changing what sort of services people actually use and also in the way people experience this support. These benefits need to be set against the costs of the services and support people used. Where benefits can be measured in $\mathrm{f}$-equivalent terms, net (monetary) benefit can be calculated as the value of benefits less the costs incurred. The cost-effectiveness of personal health budgets was assessed by comparing whether people in the personal health budget experienced greater net benefit than those people in the control group.

Benefits were measured as the change in health and care-related quality of life using the ASCOT and EQ-5D scales. These changes were converted into monetary values using pre-determined willingnessto-pay thresholds for a unit gain in quality of life over a year. As a base we used a $£ 30,000$ per QALY threshold for both ASCOT and EQ-5D. To better ensure we attribute the differences in net benefits at follow-up between the personal health budget and control groups to the use of personal health budgets, we subtracted any difference in net benefit amount that existed at baseline (i.e. prior to personal health budgets use).

After subtracting the baseline difference, the personal health budget group had ASCOT-measured net benefits of $£ 2300$ more than the control group on average (at the $£ 30,000$ threshold) comprising $£ 1180$ worth of additional benefit and $£ 1120$ less cost. The difference in net benefit between the groups was statistically significantly different from zero at the $10 \%$ significance level, suggesting with $90 \%$ confidence that personal health budgets actually produce greater net benefit than conventional arrangements. Bootstrapping confirmed that in over $90 \%$ of re-samples of the data, the corresponding net benefit difference was in the cost-effective range i.e. greater than zero (at thresholds of $£ 30,000$ or above). The corresponding difference in net benefit using a $£ 20,000$ threshold ( $€ 1910$ ) or below was not significantly different from zero. 
We did not find significant differences on the EQ-5D scale. With a $£ 30,000$ threshold EQ-5Dmeasured net benefit averaged only $£ 810$ greater in the personal health budget group than in the control group (after subtracting baseline differences). Given the variation between participants in the study, we could not be confident (at $90 \%$ or better) that this was a real difference.

Although the results suggested personal health budget to be cost-effective at borderline significance $(10 \%)$ for the whole sample, we found stronger and more significant effects for sub-groups. In particular, where personal health budgets were implemented as model 1 (i.e. with the budget known before support planning; with flexibility in what help can be purchased and where the recipient has a choice of the type of budget), they showed improvements in net benefits of a sample average $£ 4830$ over the control group, with significance at the $5 \%$ level. Furthermore, where budgets exceeded $£ 1000$ a year in value, the gain in net benefits for personal health budgets averaged $£ 4340$ in the sample which, again, is significant at the $5 \%$ level. For all types of personal health budgets we found that PHBs were cost-effective compared to controls in the NHS Continuing Healthcare and mental health sub-sample (at 10\% significance). However, the change in net benefits was no higher in the personal health budget group than in controls for the other health condition sub-groups. It is worth noting that the size of the NHS Continuing Healthcare sample was relatively small due to a number of ineligible study participants and higher mortality rates after baseline. As a result, statistical significance was low, even though effect sizes were often very high compared to other groups.

The difference-in-difference design of the evaluation removed the effects of baseline differences between personal health budget and control groups. To guard against the possibility of systematic differences in changes in costs and benefits due to baseline differences, we also used range of control factors in the net benefit analysis. These were not significant overall and did not affect the results.

A number of assumptions were made in the analysis - in particular about calculating costs and in the process of imputing missing data. 'Sensitivity analysis' involves changing the assumptions and reestimating the results. We found for all but the most extreme assumptions, that the study data showed personal health budgets to be cost-effective using the ASCOT measure. In most cases in fact, the strength of the result increased so that personal health budgets were estimated to be costeffective at the $95 \%$ confidence level.

In gauging cost-effectiveness, we assessed the overall effect of personal health budgets in the pilot. In other words, we assessed personal health budgets in terms of their actual implementation in the study, including choices about resourcing PHBs. It is possible, nonetheless, that personal health budgets could be deployed without changes in resource levels. We therefore conducted further analyses to estimate effect size as if the level of expenditure on services and support was the same between personal health budget and control groups. To begin with, these analyses suggested that the use of a personal health budget has a direct impact on quality of life via improved choice, control and tailoring of services to person need and circumstances - the 'control/tailoring' effect.

The analysis also showed that personal health budgets have an impact on expenditure levels, both as a result of a change in service need and also as a consequence of changed resourcing levels. In turn, a change in expenditure (i.e. a change in service use) has an impact on quality of life, other things 
equal. So, where personal health budgets lead to a change in service use/expenditure, they also have a knock-on effect on quality of life. This latter effect goes some way to offsetting the direct 'control/tailoring' effect, depending on whether we use the ASCOT or EQ-5D measure.

The EQ-5D measure is not especially sensitive to measuring the benefits of improved control and tailoring, particularly the value of choice and control. It is perhaps not surprising, therefore, that the service effects tended to dominate on EQ-5D leading to zero overall effect. ASCOT, by contrast, is designed for people with long-term conditions, where choice and control in managing one's condition is highly valued. In this case, the 'control/tailoring' effect was greater than the 'service resourcing' effect, which is why we found that personal health budgets were generally cost-effective on the ASCOT scale but not with EQ-5D.

Cost-effectiveness was assessed in this chapter in terms people's use of the health and social care systems' resources. In practice, we might expect wider implications of using personal health budgets. The impact on families and carers of personal health budgets is considered in the next chapter, although we do not explicitly build this into our cost-effectiveness analysis. Furthermore, in this chapter we have looked at cost-effectiveness on the basis of people that survived to follow-up. It is theoretically possible to include people that died during the analysis as that both the EQ-5D and ASCOT scales have death-equivalent quality of life ratings (of 0 ). After death, service utilisation is also clearly zero. The problem is that we do not know the profile of outcomes or service use in the followup period prior to death. We expect these profiles to be quite different to those people that did not die. As reported in the previous chapter, we found no significant difference in mortality rate between groups which mitigates the consequences of this omission to a certain extent. It remains the case, nonetheless, that we cannot know whether there were any systematic differences in service use or outcome between the groups prior to death.

This was a highly complex evaluation that involved a substantial data collection from a population of people with significant long-term health conditions and with a follow-up period of a year or more. Not surprisingly there was missing data and loss of participants to follow-up. It was therefore especially important to use multiple imputation techniques and to ensure that those techniques were well specified for this dataset. As with any statistical method, some assumptions had to be made using multiple imputation. As noted we tested the sensitivity of our results using an alternative imputed dataset, alternative imputation models, different sub-samples reflecting missing data patterns, and different costing assumption. We found very similar - if not stronger - results. Sensitivity analyses of this kind provide more confidence in the main results of the study.

\subsection{Annex to chapter 6: Sensitivity analysis}

\subsubsection{Imputation assumptions}

Multiple imputation (MI) involves using the full set of data in the sample to infer or impute missing values of certain variables. Statistical relationships are estimated for this purpose and link the variables in the dataset. Multiple imputation uses chains of these estimated equations to build up a full imputed dataset using an iterative feedback process. The basic idea is that there are underlying patterns in the dataset that can be exploited to fill in missing data - essentially the whole is greater 
than the sum of the parts. However, because these are statistical processes, they are subject to statistical noise. Actual values estimated to replace missing values are subject to some 'error' and also the randomness in the process that resolves a statistical probability into a specific value.

To reflect this statistical noise, multiple imputation produces a set of parallel samples of the data with slightly different values of missing data. Any analysis of the data is made on each dataset and, after variations between the datasets are accounted for, the results are combined. A practical consideration is how many imputations of the dataset to use. Rubin (1987), the originator of the multiple imputation technique, showed that five imputations were often sufficient but that there are no hard-and-fast rules. There is also the choice of the starting values for the randomness process (the randomness 'seed') which in theory should not affect the imputation results in a large dataset. Finally there are choices to be made about the imputation 'model'. A relevant consideration here how 'censored' variables are modelled; these are variable which cannot take certain values - for example we cannot have negative costs. Stata offers two approaches in this regard: truncated regression and predictive mean matching (PMM). Our base models use truncated regression and so as part of our sensitivity analysis we instead use the PMM approach.

To test the sensitivity of the main results, we added a further five imputations to our main dataset with a different randomly selected seed value. The main analyses were then re-produced using the ten imputations dataset. The key results are presented in Figure 6-14 and Figure 6-15 below, comparing results from the base five imputations dataset with the 'sensitivity' results where an additional five imputations are added. The figures show difference-in-difference results for NMB using ASCOT and EQ-5D scales respectively. The results are very similar for the two datasets with, if anything, slightly tighter confidence intervals than for the ten imputations dataset. 
Figure 6-14. ASCOT NMB Diff-in-Diff, comparing base MI and sensitivity MI results, point estimates and confidence intervals

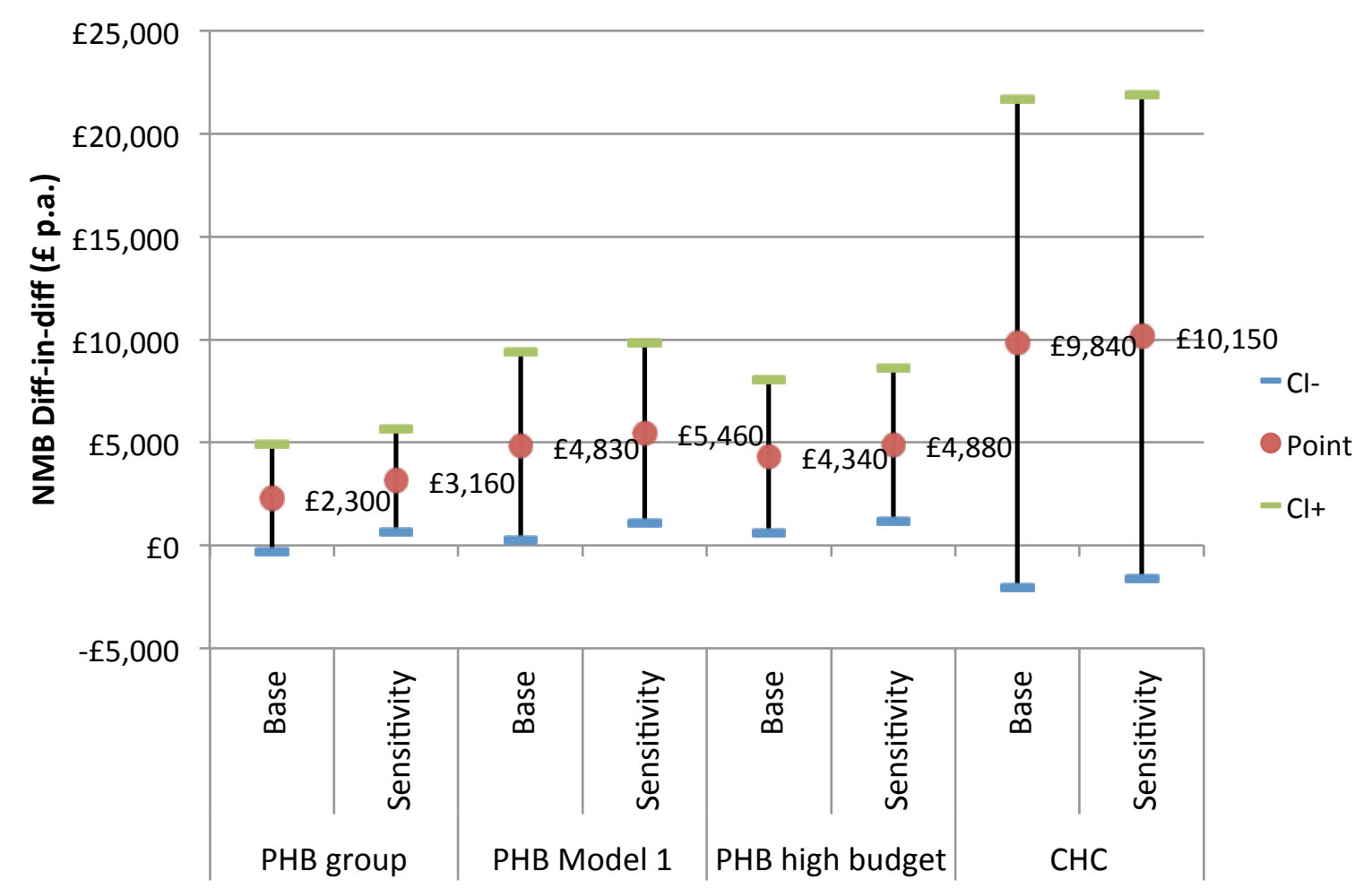

Figure 6-15. EQ-5D NMB Diff-in-Diff, comparing base MI and sensitivity MI results, point estimates and confidence intervals

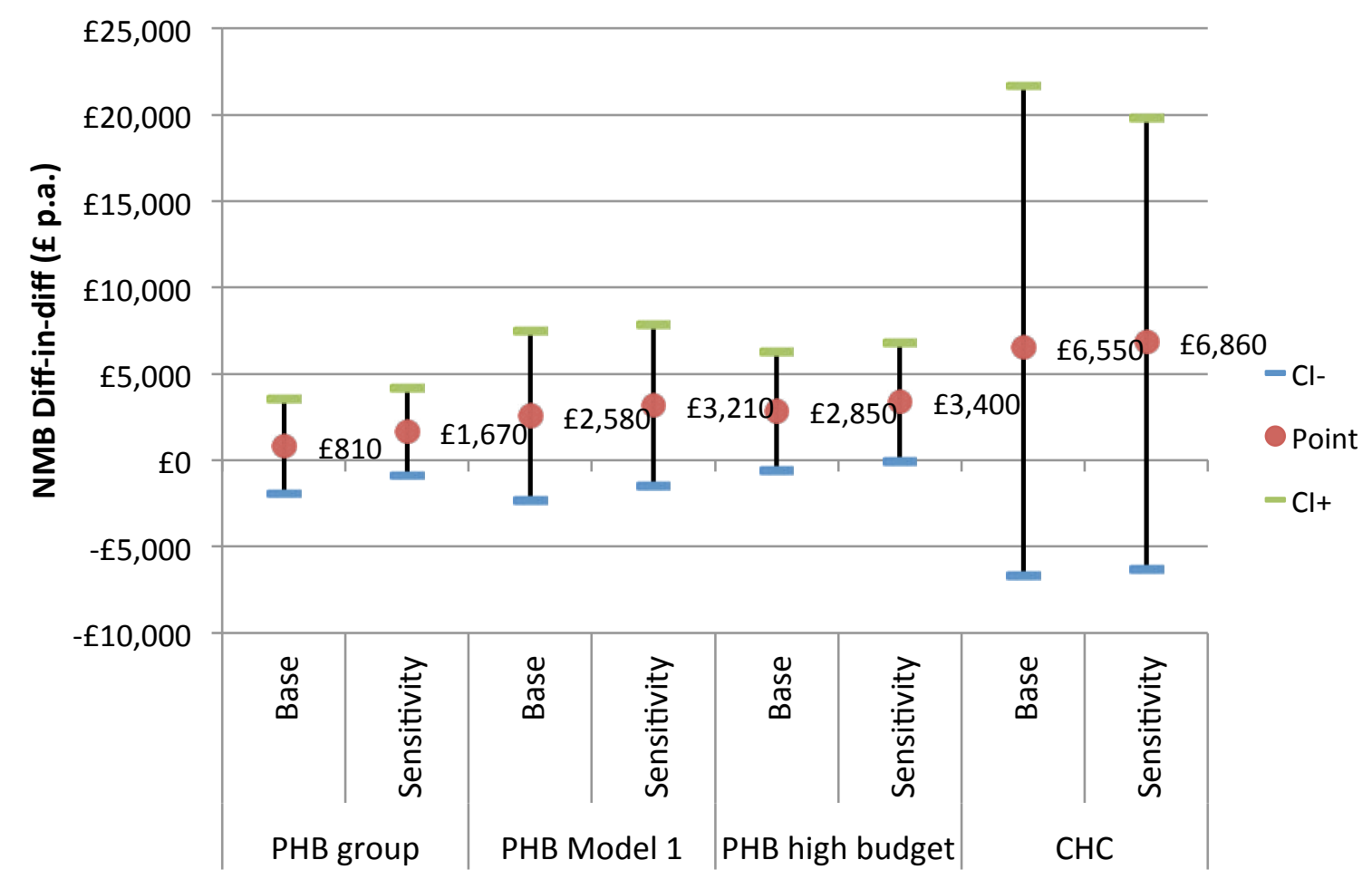


Ml estimation accounts for a proportion of missing data. In general, a high proportion of missing values will generate higher between imputation variance (more noise) than a low proportion of missing values. Nonetheless, we also tested the main analyses on sub-samples of the full dataset selected according to the availability of actual follow-up data. The results are shown in Table 6-19. Although there are some small differences in estimation coefficients and significance levels, which are to be expected, the overall nature of the results is largely unaffected.

Table 6-19. Sensitivity of results to data sub-samples

\begin{tabular}{llccc}
\hline & \multicolumn{2}{l}{$\begin{array}{l}\text { Only specific outcome } \\
\text { data at follow-up }\end{array}$} & \multicolumn{2}{l}{ Any follow-up data } \\
& $\begin{array}{l}\text { Coeff } \\
\text { PHB any }\end{array}$ & prob & Coeff & prob \\
\hline High Budget & 1751 & 0.134 & 1997 & $0.089^{*}$ \\
\hline PHB model 1 & 3687 & $0.084^{*}$ & 3752 & $0.034^{* *}$ \\
Mental health & 5104 & $0.032^{*}$ & 4396 & $0.052^{*}$ \\
\hline NHS Continuing Healthcare & 9551 & $0.066^{*}$ & 4459 & 0.111 \\
\hline Sample size & 5118 & $0.095^{*}$ & 9644 & 0.124 \\
\hline Percentage of full sample & $72 \%$ & & & \\
\hline
\end{tabular}

Significance levels: ${ }^{*} p<0.10^{* *} p<0.05^{* * *} p<0.001$

Using PMM estimations rather than truncated regression in the imputation model made very little difference to the point effect sizes when comparing net benefit for the personal health budget and control groups. It did appear, however, to produce smaller standard errors and therefore results that were significant at higher confidence levels - see Figure 6-16 (ASCOT) and Figure 6-17 (EQ-5D). For example, in using PMM in the imputation, we estimated that net benefits were $£ 2310$ higher for the personal health budget group compared to the control group, after baseline subtraction (rather than f2300 in the base model). This new estimate was significant at the $95 \%$ confidence level (rather than the $90 \%$ level). Also, the high-value personal health budget group had net benefits of $£ 2760$ greater than the control group, when measured using the EQ-5D scale, which was significant at the $95 \%$ confidence level. The largest difference in the sensitivity results were for the NHS Continuing Healthcare sub-group, reflecting the relatively small sub-sample size and the high volatility in costs for this group. Effect sizes in this case were reduced, but so were the standard errors compared to the base case. The net benefit difference (after baseline) remained significant at the $90 \%$ confidence level for ASCOT and insignificant for EQ-5D-measured net benefit. 
Figure 6-16. ASCOT NMB Diff-in-Diff, comparing MI models (truncated regression is base against $P M M)$, point estimates and confidence intervals

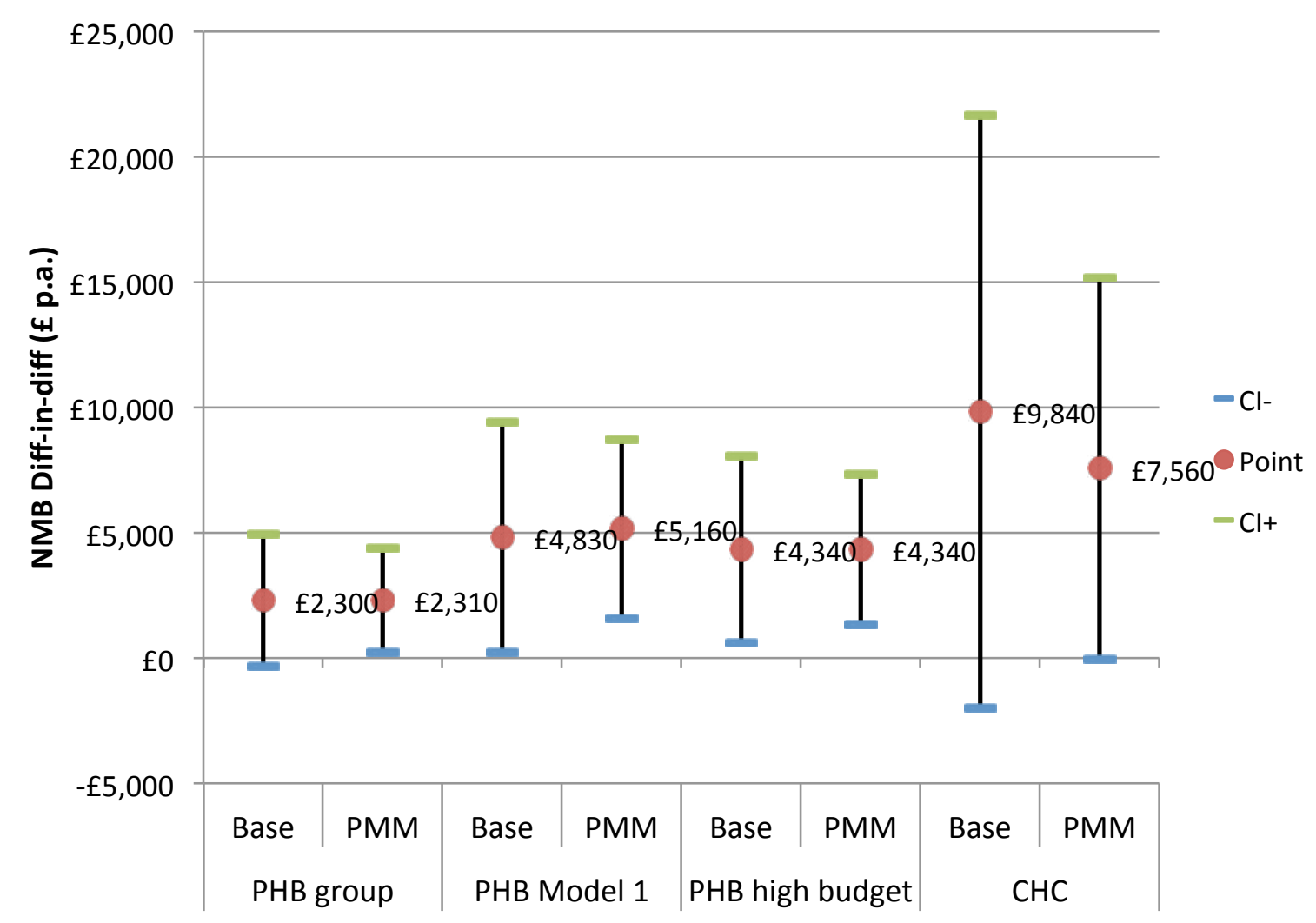


Figure 6-17. EQ-5D NMB Diff-in-Diff, comparing MI models (truncated regression is base against $P M M)$, point estimates and confidence intervals

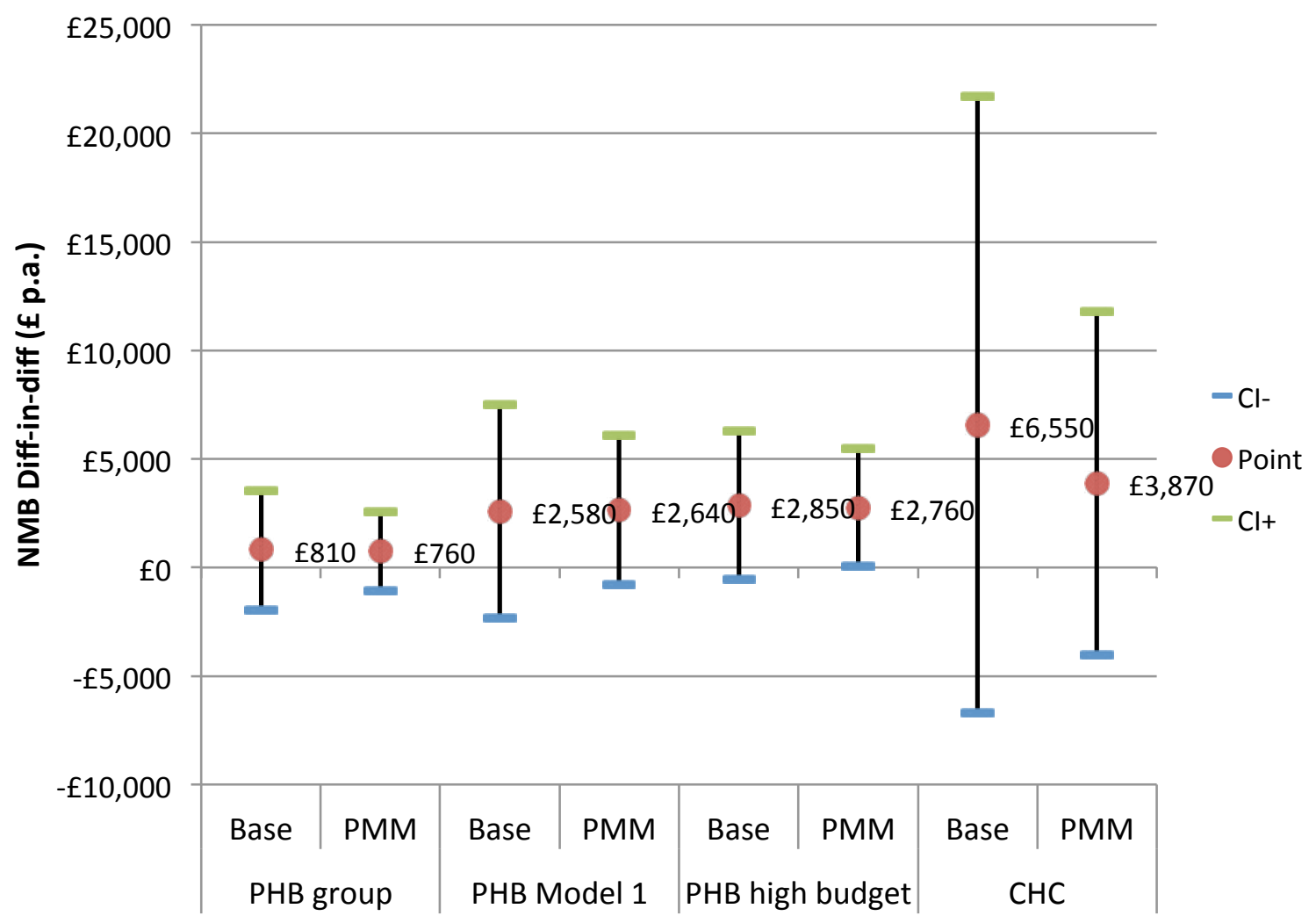

\subsubsection{Cost assumptions}

The main cost assumption concerns the threshold at which personal health budgets are regarded as funded by substitution rather than by additional resources. In the main analysis we assumed a threshold of $£ 1000$ per annum: that is, personal health budgets of $£ 1000$ or less in each service category were provided in addition to convention services in that category. Budgets over $£ 1000$ for each category of service were provided as a substitute for conventional service in that category.

To test the sensitivity of this assumption we re-estimated the models with a $£ 500$ limit instead. We also re-estimated with no limit, meaning that the personal health budget substituted for all service categories. This latter option is very unlikely to be valid in practice and is provided only for comparison. 
Figure 6-18. ASCOT NMB Diff-in-Diff, comparing base cost assumption ( $f 1000$ limit) with the

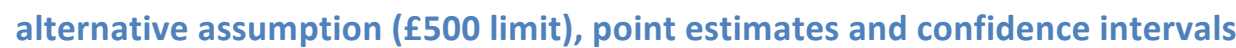

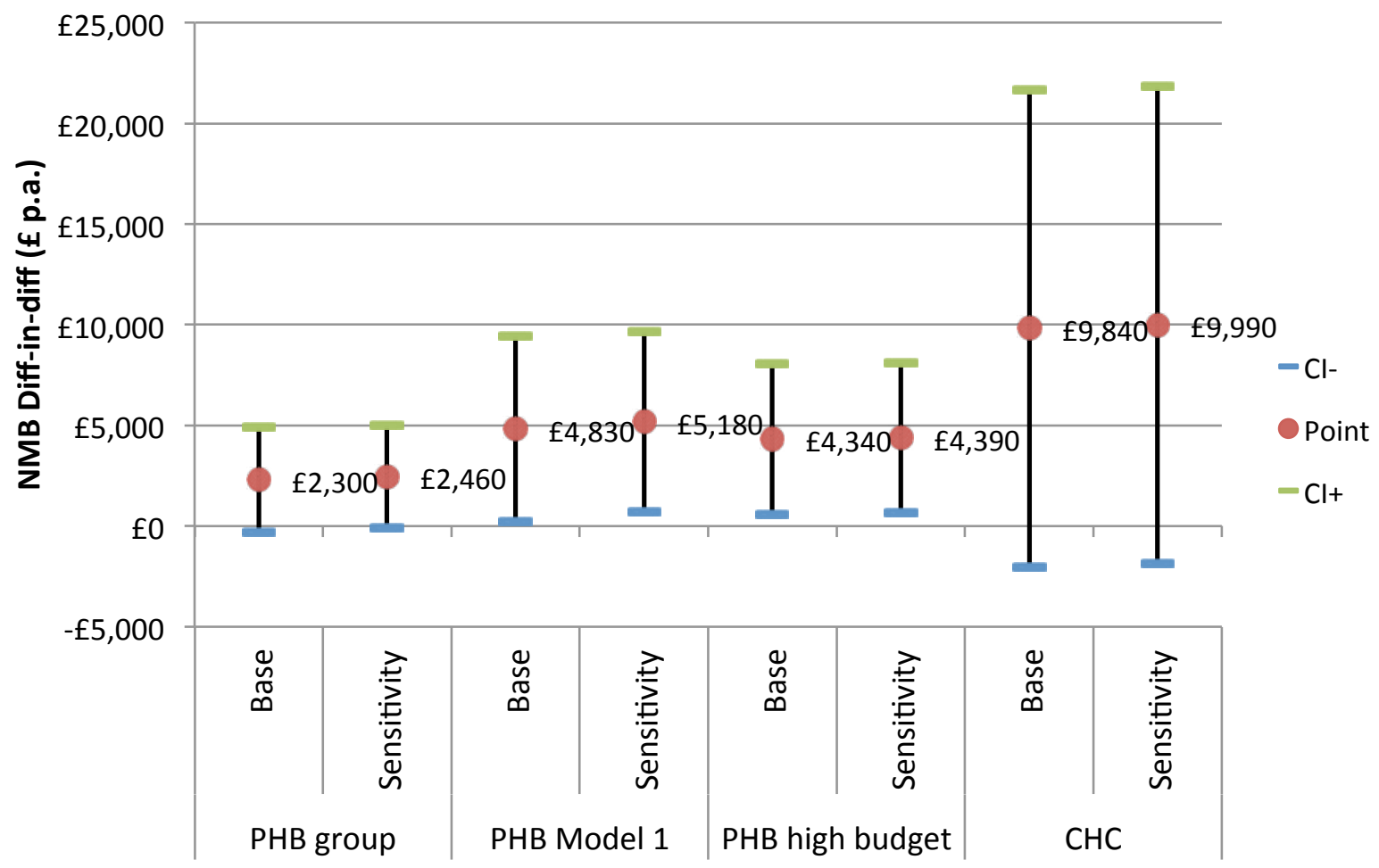

Figure 6-19. EQ-5D NMB Diff-in-Diff, comparing base cost assumption (f1000 limit) with the alternative assumption ( $\mathrm{f500}$ limit), point estimates and confidence intervals

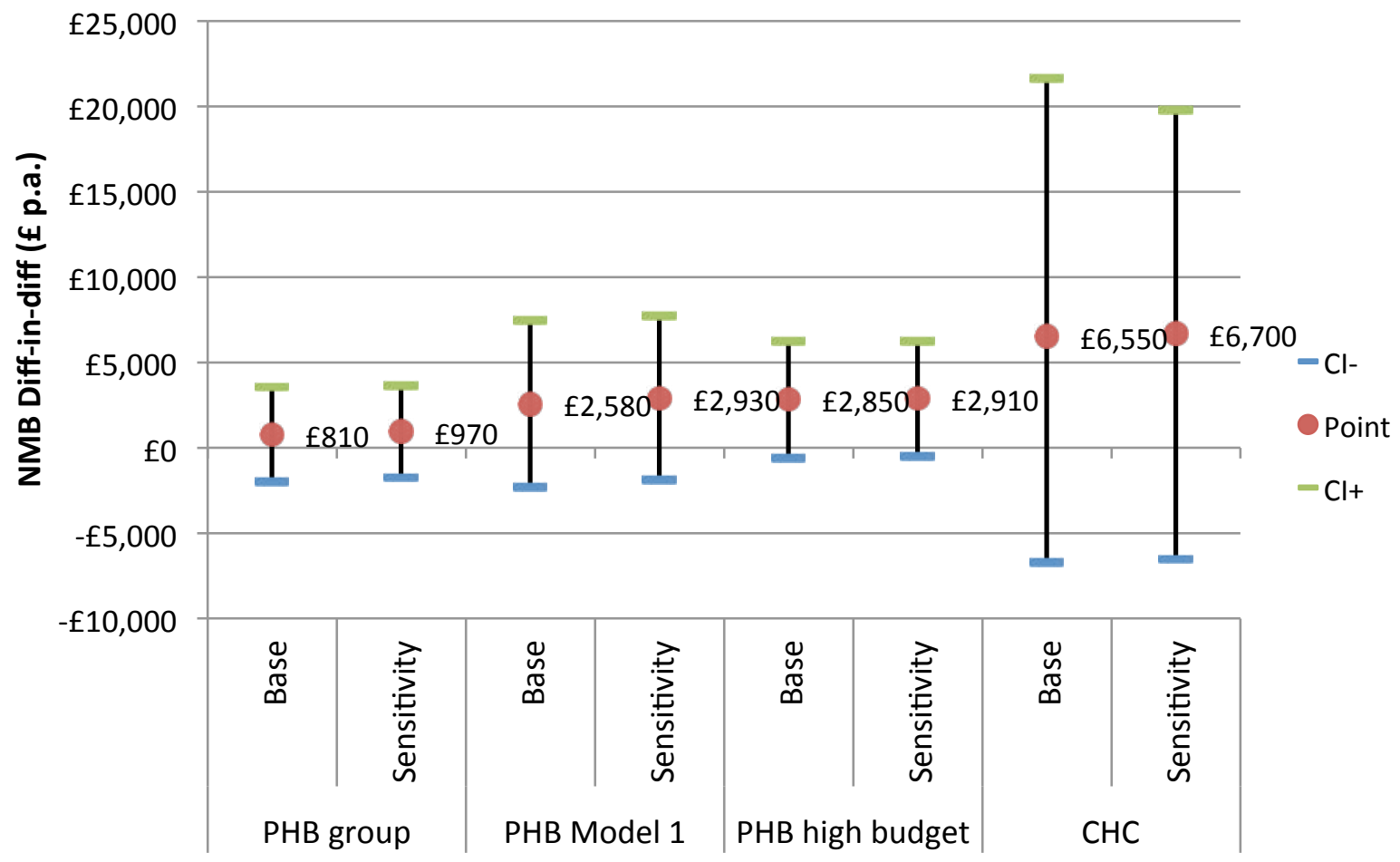


Figure 6-20. ASCOT NMB Diff-in-Diff, comparing base cost assumption ( $f 1000$ limit) with the alternative assumption ( $\mathrm{f} 0$ limit), point estimates and confidence intervals

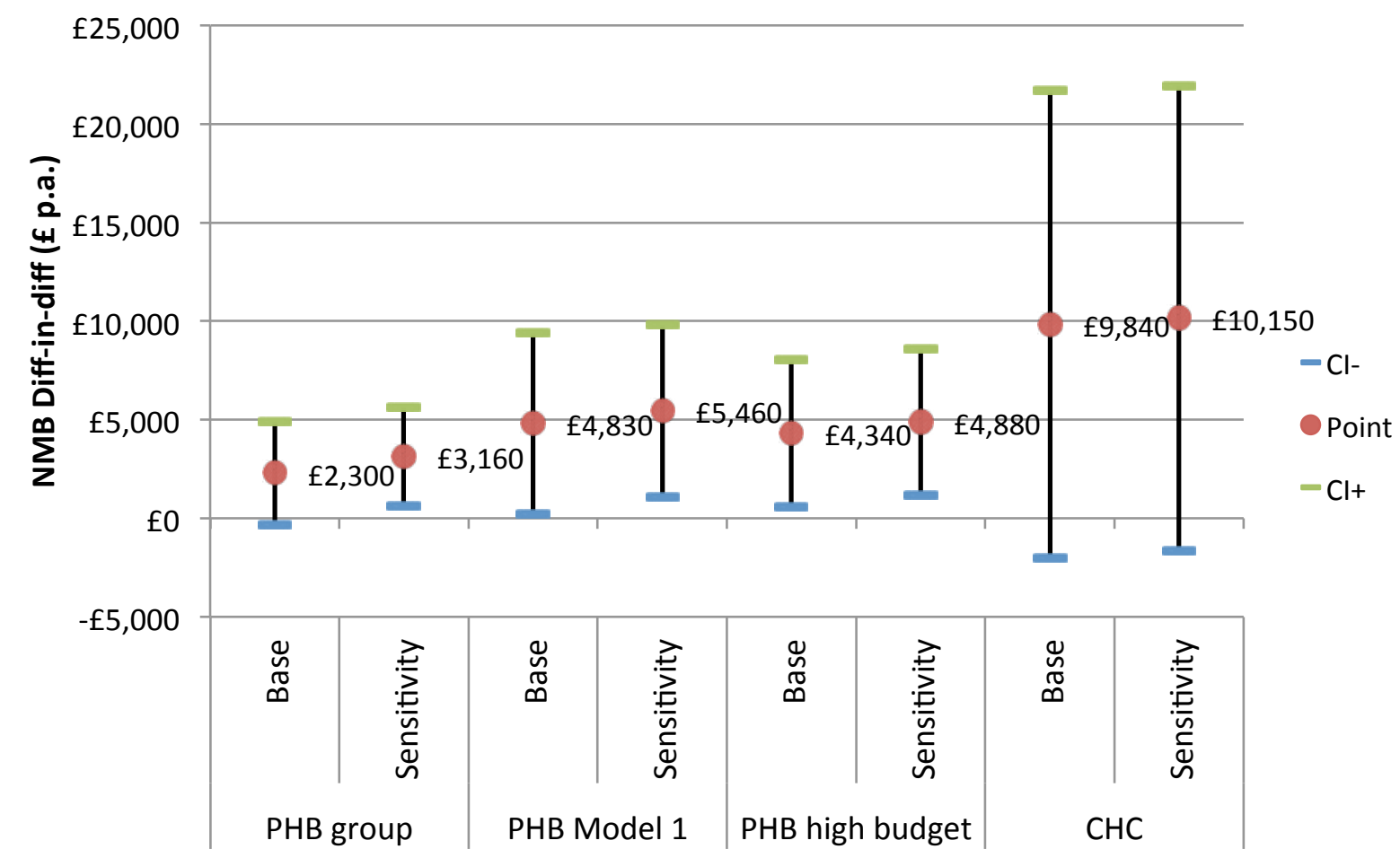

Figure 6-21. EQ-5D NMB Diff-in-Diff, comparing base cost assumption ( $f 1000$ limit) with the alternative assumption ( $\mathrm{f0}$ limit), point estimates and confidence intervals

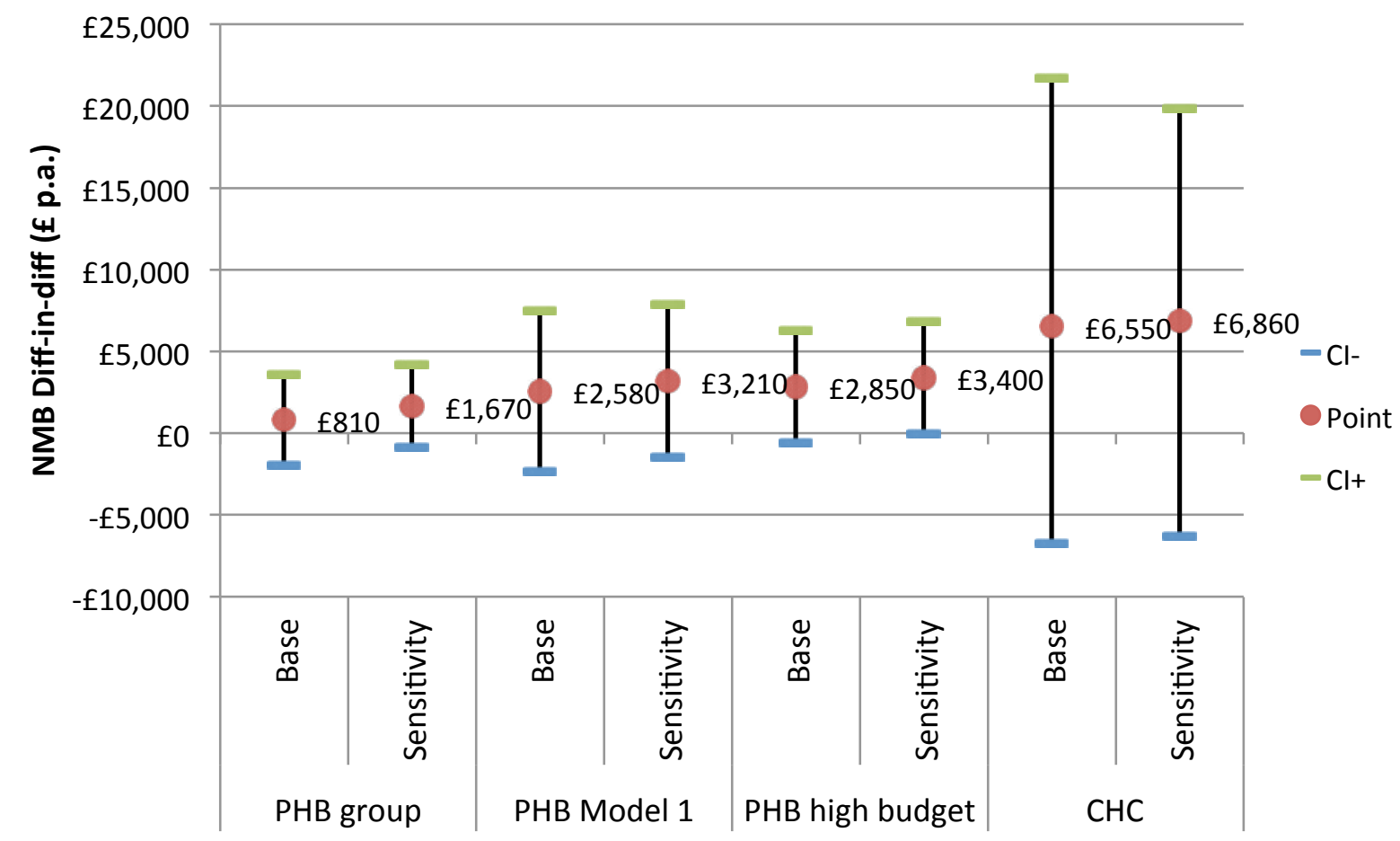




\section{The impact of receiving personal health budgets on carers}

\subsection{Summary}

An important element of the evaluation was to explore whether personal health budgets had an impact on informal care and on the caring role, compared to conventional service delivery. There were three aims:

- To explore whether personal health budgets had an impact on participants' receipt of informal care.

- To explore the perceptions held by informal carers who provided help to participants in both the personal health budget and the control group.

- To explore whether personal health budgets had an impact on carers' quality of life and the caring role, compared with conventional service delivery.

In total, 147 questionnaires were received from the sample of 282 carers (52\%). Of these, 88 were caring for a participant in the personal health budget group and 59 for a participant in the control group.

From the difference-in-difference multivariate analysis, a number of key results were found:

- In the whole sample analysis we did not find a significant difference in the reported use of informal care between personal health budget and control groups.

- Personal health budgets using implementation model 3 were associated with an increase in informal care receipt inside the household relative to controls.

- Personal health budgets of model 1 type were associated with a decrease in informal care receipt from outside the household relative to controls.

- We also found that personal health budgets of model 2 type were associated with an increase in informal care receipt inside the household relative to controls.

This pattern of results does not entirely accord with our prior expectation that personal health budgets would reduce the need for people to rely on informal care.

Analysis involving the data from the carer outcome questionnaire revealed:

- Carers providing assistance to an individual in the personal health budget group were more likely to report better quality of life and perceived health compared to carers assisting an individual in the control group.

- Carers in the personal health budget group generally reported lower instances of having their health affected by their caring role.

- Carers seemed to be satisfied with the personal health budget process in terms of support planning, the amount of the budget and the amount of help that was offered when deciding what services or support to purchase from the personal health budget.

- The findings need to be treated with caution due to the sample size in the sub-group analysis. 


\subsection{Introduction}

An important element of the evaluation was to explore whether personal health budgets had an impact on informal care and on the caring role, compared to conventional service delivery. Three questions will be addressed in this chapter:

- Is there evidence to suggest that personal health budgets have a significant impact on informal care receipt at follow-up compared to conventional service delivery?

- What factors are associated with a change in informal care receipt?

- Is there evidence to suggest that personal health budgets had an impact on carers' quality of life and the caring role, compared with conventional service delivery?

This chapter is structured as follows. In the next section we briefly review the methods that we used to explore the effects of using a personal health budget on carers. Section 7.4 reports the results of the analysis of receipt of informal care. Section 7.5 presents the results of the analysis of carer impact and we end with a discussion of those results in Section 7.6.

\subsection{Method}

One of the aims of the overall study was to explore whether personal health budgets had an impact on participants' receipt of informal care. The baseline and main follow-up outcome questionnaires asked whether participants received practical help on a regular basis from any friends, neighbours, partner or family member who lived in or outside the service user's household. The questionnaire also asked about the number of different people who provided support, and the relationship the participant had with people who provided support.

An additional aim of the study was to explore the perceptions held by informal carers who provided help to participants in both the personal health budget and the control group. The data collection included sending a postal questionnaire to carers of participants recruited to the main study 12 months after the date of consent. At the time of recruitment the participant was asked whether they would give permission for their carer to be contacted. The questionnaire included a number of outcome measures to explore whether personal health budgets had an impact on carers' quality of life and the caring role. Appendix A describes the measures used in more detail, and here we briefly summarise them.

- Health-related quality of life: The EQ-5D utility scale aims to measure a person's quality of life in domains likely to be related to their underlying health status. It measures personal functioning (as potentially constrained by poor health). We use the three-level version in this study.

- Care-related quality of life: The scale was based on the first national survey of adult carers' experiences, views and outcomes conducted in England by councils with social services responsibilities (Holder et al., 2009; Malley et al., 2010; Fox et al., 2010). 
- Perceived quality of life: The seven-point scale with categories ranging from 'So good, it could not be better' to 'So bad, it could not be worse'.

- Perceived health: The five-point scale asks respondents to rate their health in general according to five categories ranging from 'Very good' to 'Very bad'.

The outcome questionnaire also contained a number of socio-demographic and socio-economic questions which will be controlled for in the multivariate analysis.

The quantitative arm of the study was supplemented with qualitative data derived from semistructured interviews with a sub-sample of carers. These interviews were conducted three and nine months after the offer of a personal health budget was made to the cared-for person. Semistructured interviews were conducted only with carers of someone in receipt of a personal health budget.

\subsubsection{Response rates}

The initial analysis was run on the main imputed dataset to explore variation in informal care receipt between baseline and follow-up (See Appendix C, section C-7 for details).

The dataset containing responses from carers was used to explore whether personal health budgets had an impact on their quality of life and the caring role. This analysis was followed by running analysis on the dataset containing responses from carers. At the time of recruitment to the main study, 282 participants agreed that their carer could be contacted by the research team. The following processes were followed with the aim of obtaining the best possible sample of carers for this aspect of the study.

1. A postal questionnaire was sent to carers 12 months after the cared-for person had consented to participate in the study;

2. A reminder questionnaire was sent to non-respondents five weeks after the initial questionnaire was sent to carers;

3. Where a participant's consultee was a carer, an invitation letter and questionnaire were sent to ask whether they would be willing to participate in the study. ${ }^{41}$

\footnotetext{
${ }^{41}$ To reduce burden on carers and increase response rates, not all questions were included in reminder questionnaires and questionnaires to consultees. This is reflected in the responses to some questions.
} 


\subsubsection{Analyses of responses}

The difference-in-difference approach was followed to allow us to explore whether personal health budgets had an impact on receipt of informal care compared to conventional service delivery. The analysis explored whether the average change in informal care receipt in the personal health budget group was higher than the average change in the control group. A number of confounding factors were used in the analysis to control for any differences in the characteristics of individuals within the personal health budget and control groups. Furthermore, the impact that personal health budget implementation models had on explaining changes to informal care receipt was explored in the multivariate difference-in-difference analysis.

This analysis will be followed by exploring whether personal health budgets had an impact on carers' perceptions of quality of life, health and their caring role. However, the extent to which we can explore sub-groups is limited due to the sample size of carers participating in the study.

\subsection{Variation in informal care receipt between baseline and follow-up}

The initial analysis explored the variation in informal care receipt between baseline and follow-up. Table 7-1 shows there was an increase in receiving informal care at follow-up in both groups, although the change was lower among individuals in the personal health budget group. However, potentially baseline differences could account for some of the difference in change, rather than receiving the personal health budget. For this reason we also estimated the difference in the change over time when controlling for a range of baseline characteristics.

Table 7-1 Informal care receipt-sample means and differences, baseline and follow-up

\begin{tabular}{llcccc}
\hline & & \multicolumn{2}{c}{ PHB Group } & \multicolumn{2}{c}{ Control Group } \\
& & Mean & SD & Mean & SD \\
\hline $\begin{array}{l}\text { Informal care within } \\
\text { household }\end{array}$ & Baseline & .565 & .496 & .474 & .499 \\
& Follow-up & .572 & .495 & .520 & .499 \\
& Change & .006 & .469 & .043 & .485 \\
\multirow{2}{*}{$\begin{array}{l}\text { Informal care } \\
\text { outside household }\end{array}$} & Baseline & .412 & .492 & .364 & .481 \\
& Follow-up & .663 & .473 & .699 & .459 \\
\cline { 2 - 5 } No informal care & Change & .249 & .756 & .335 & .733 \\
& Baseline & .197 & .397 & .268 & .443 \\
& Follow-up & .139 & .345 & .144 & .351 \\
& Change & -.052 & .536 & -.115 & -.115 \\
\hline
\end{tabular}

Table 7-2 shows that the difference in receiving informal care between baseline and follow-up was associated with baseline dependency levels and health conditions. The non-significant interaction effects with the group variable (personal health budget or control group) indicate that receiving a personal health budget did not have a significant impact on informal care receipt. 
Table 7-2 Direction of change in receipt of informal care, with control factors

\begin{tabular}{|c|c|c|c|c|c|c|}
\hline & \multicolumn{2}{|c|}{$\begin{array}{l}\text { Informal care receipt } \\
\text { inside the household }\end{array}$} & \multicolumn{2}{|c|}{$\begin{array}{l}\text { Informal care receipt } \\
\text { outside the household }\end{array}$} & \multicolumn{2}{|c|}{ No informal care receipt } \\
\hline & Coeff & Prob & Coeff & Prob & Coeff & Prob \\
\hline PHB group & .019 & .807 & -.068 & .256 & .024 & .690 \\
\hline Age & .003 & .457 & -.001 & .707 & -.000 & .871 \\
\hline Male & -.079 & .244 & .142 & $.007^{* *}$ & -.063 & .261 \\
\hline ADL score & -.022 & .198 & -.031 & $.025^{* *}$ & .052 & $.002^{* *}$ \\
\hline ADL score (sqrd) & .001 & $.033^{* *}$ & .002 & $.000^{* * *}$ & -.003 & $.000^{* * *}$ \\
\hline \multicolumn{7}{|l|}{ Health condition } \\
\hline $\begin{array}{l}\text { NHS Continuing } \\
\text { Healthcare }\end{array}$ & -.495 & $.025^{* *}$ & .073 & .875 & .309 & .455 \\
\hline Stroke & -.348 & .220 & .072 & .887 & .370 & .422 \\
\hline Diabetes & .009 & .974 & .175 & .714 & -.063 & .883 \\
\hline Mental health & -.531 & $.045^{* *}$ & -.013 & .979 & .318 & .464 \\
\hline COPD & -.370 & .144 & -.015 & .975 & .316 & .465 \\
\hline Neurological & -.496 & $.048^{* *}$ & .129 & .790 & .349 & .413 \\
\hline Follow-up period & -.001 & .315 & .000 & .749 & .000 & .783 \\
\hline Consent date & .000 & .569 & .000 & .344 & -.000 & .641 \\
\hline Area cost adjust & -.028 & .968 & .356 & .370 & -.321 & .411 \\
\hline Constant & 3.73 & .713 & 5.93 & .376 & -4.94 & .559 \\
\hline $\mathrm{N}$ & 2235 & & 2235 & & 2235 & \\
\hline Model F & 4.16 & $.000^{* * *}$ & 3.97 & $.000^{* * *}$ & 9.87 & $.000^{* * *}$ \\
\hline
\end{tabular}

Significance levels: ${ }^{*} p<0.10^{* *} p<0.05^{* * *} p<0.001$

As reported earlier, five implementation models were developed based on the findings from the indepth qualitative interviews with organisational representatives (see Table 1-2). The models were classified in terms of whether pilot sites informed budget holders of the amount in the personal health budget before support planning began; the degree of flexibility in what services/help could be purchased through the budget; and the degree to which different deployment options were offered when the decision on how to manage the budget was made. The analysis highlights a significant impact on informal care receipt after controlling for confounding variables. Personal health budgets of model 3 type were associated with an increase in informal care receipt inside the household relative to controls. Personal health budgets of model 1 type were associated with a decrease in informal care receipt from outside the household relative to controls. We also found that personal health budgets of model 2 type were associated with an increase in informal care receipt inside the household relative to controls. This pattern of results does not entirely accord with our prior expectation that personal health budgets would reduce the need for people to rely on informal care. 
Table 7-3 Direction of change in receipt of informal care, with implementation models

\begin{tabular}{lccccccc}
\hline & \multicolumn{2}{c}{$\begin{array}{l}\text { Informal care receipt } \\
\text { inside the household }\end{array}$} & \multicolumn{2}{c}{ Informal care receipt } & \multicolumn{2}{c}{ No informal care receipt } \\
& Coeff & Prob & Coeff & Prob & Coeff & Prob \\
\hline Model 1 & -.150 & .124 & -.191 & $.011^{* *}$ & .073 & .362 \\
Model 2 & .030 & $.023^{* *}$ & -.084 & .367 & .035 & .693 \\
Model 3 & .236 & $.076^{*}$ & -.035 & .750 & -.152 & .166 \\
Model 4 & -.100 & .333 & .009 & .932 & .103 & .325 \\
Model 5 & $.032^{* * *} \mathrm{p}<0.001$ & .727 & -.144 & $.024^{* *}$ & .056 & .398 \\
\hline Significance levels: ${ }^{*} \mathrm{p}<0.10^{* *} \mathrm{p}<0.05^{*}$ & & & &
\end{tabular}

The next section will explore whether there was an impact on carers' quality of life and caring role.

\subsection{The impact of personal health budgets on carers}

In total, 147 questionnaires were received from the sample of 282 carers (52\%). Of these, 88 were caring for a participant in the personal health budget group and 59 for a participant in the control group. The majority of carers were aged 60 years or over $(58 \%, n=83)$ followed by carers aged $45-59$ $(32 \%, n=46)$. The remaining carers in the sample were aged $16-24(1 \%, n=2), 25-34(4 \%, n=5)$ and $35-$ $44(6 \%, n=8)$. Three people declined to answer.

Table 7-4 shows the spread of health conditions in terms of the carers' looked-after participants. Notably, there are no carers for any participants with diabetes as their primary health condition. Other than diabetes, carers for participants with COPD were the least represented in both the personal health budget and control groups. The research team relied on participants in the main study to consent for their carer to be contacted.

Table 7-4 Health condition of the cared for participant

\begin{tabular}{lcc}
\hline Primary Health condition & $\begin{array}{c}\text { PHB Group } \\
\text { \% (N) }\end{array}$ & $\begin{array}{c}\text { Control Group } \\
\%(\mathbf{N})\end{array}$ \\
\hline Stroke & $15 \%(13)$ & $26 \%(15)$ \\
Mental health & $20 \%(17)$ & $10 \%(6)$ \\
COPD & $5 \%(4)$ & $9 \%(5)$ \\
Neurological condition & $38 \%(33)$ & $34 \%(20)$ \\
Continuing healthcare & $22 \%(19)$ & $21 \%(12)$ \\
Total & $100 \%(86)$ & $100 \%(58)$ \\
\hline
\end{tabular}

A number of semi-structured interviews were also conducted with carers of personal health budget holders, and evidence gathered from these will be drawn on throughout this chapter. Chapter 3 provides a full description of the carers that participated in the in-depth qualitative interviews.

Within the sample of carers, $71 \%(n=45)$ were caring for a budget holder who was receiving support/services paid for by the personal health budget. Of these, $49 \%(n=22)$ had been receiving their personal health budget for more than six months. Almost three quarters had been receiving 
their budget for at least three months. Some participants either had nothing in place at all, did not have all of their budget in place, or had had their budget in place for a month or less $(8 \%, n=4)$.

Despite the delays within the implementation process, overall the personal health budget process did appear to have an impact on carers' aspirations. Table 7-5 explores the response among carers when asked whether or not they thought the personal health budget had changed their view of what could be achieved by the person they cared for. It shows that $42 \%(n=31)$ of carers stated that their view had changed 'a lot', 39\% ( $n=29)$ stated that their view had changed 'a little', while 19\% ( $n=14)$ stated that their view had not changed at all.

Table 7-5 Changed view of what cared for person can achieve

\section{$\%(N)$}

\begin{tabular}{ll}
\hline $\begin{array}{l}\text { Has the personal health budget changed your view } \\
\text { of what cared for person can achieve? }\end{array}$ \\
\hline A lot & $42 \%(31)$ \\
\hline A little & $39 \%(29)$ \\
\hline Not at all & $19 \%(14)$ \\
\hline
\end{tabular}

Table 7-6 shows the trend in satisfaction levels expressed by carers. This table indicates that carers were generally satisfied with the amount of the budget that the person they cared for received $(76 \%$, $\mathrm{n}=31$ ) and that the majority of carers were also satisfied with the knowledge of the support planner $(74 \%, n=32)$. When asked whether they thought the cared-for person had enough help with deciding what to spend their personal health budget on, $86 \%$ of carers agreed $(n=30)$. A majority of carers $(78 \% ; n=66)$ expressed a degree of satisfaction with the support planning process, and more carers in the personal health budget group $(29 \%, \mathrm{n}=22)$ reported that it was 'very easy' to get the support or services they needed in place compared to carers in the control group $(13 \%, n=6)$. All results in the table did not reach statistical significance due to the small sample sizes. Percentages may not equal 100 due to rounding.

Table 7-6 Satisfaction and support planning process (personal health budget group)

\begin{tabular}{lccc}
\hline & $\begin{array}{c}\text { Satisfaction with } \\
\text { amount of budget }\end{array}$ & $\begin{array}{c}\text { Satisfaction with } \\
\text { knowledge of the care } \\
\text { planner }\end{array}$ & $\begin{array}{c}\text { Support planning } \\
\text { process }\end{array}$ \\
\hline Extremely satisfied & $24 \%(10)$ & $19 \%(8)$ & $14 \%(12)$ \\
Very satisfied & $29 \%(12)$ & $26 \%(11)$ & $25 \%(21)$ \\
Quite satisfied & $22 \%(9)$ & $30 \%(13)$ & $39 \%(33)$ \\
Neither satisfied nor dissatisfied & $12 \%(5)$ & $7 \%(3)$ & $6 \%(5)$ \\
Quite dissatisfied & $2 \%(1)$ & $9 \%(4)$ & $6 \%(5)$ \\
Very dissatisfied & $5 \%(2)$ & $2 \%(1)$ & $2 \%(2)$ \\
Extremely dissatisfied & $5 \%(2)$ & $7 \%(3)$ & $7 \%(6)$ \\
Total & $100 \%(41)$ & $100 \%(43)$ & $100 \%(84)$ \\
\hline
\end{tabular}


The 13 carers that took part in the in-depth interviews had mixed experiences of implementation and support planning. One carer reported clear benefits from her lead role in planning how her son's budget would be used:

"It did me good because I actually sat down over a few days and wrote out a list of things. ... When you're a carer you're so wrapped up in just trying to get through the day and the next day, you don't really think outside the box. It kind of threw a spanner in the works for me really ... but it was good for me to think outside the box and it's made me think outside the box in other ways" (carer).

One or two carers reported receiving a great deal of help with the recruitment and on-going employment of paid carers to be funded by the personal health budget where this was required. In addition, carers of people with a long-term neurological condition, and in particular those eligible for NHS Continuing Healthcare, described how their own needs were also addressed as part of the care planning process:

"It takes the pressure off of me. I'll get a break which'll mean that I'm not tired all the time... and I think that's better for [son] as well that I'm not stressed out all the time" (carer).

Around half of the carers interviewed for the in-depth arm of the study had experienced difficulties or delays in decision-making about the size of their relative's budget, agreeing care/support plans, or setting up efficient payment arrangements. Indeed, three of the 13 carers still did not have a budget in place at the time of their nine-month interview. The problems mainly affected carers supporting people with long-term neurological conditions or who were eligible for NHS Continuing Healthcare. Some problems appeared to reflect difficulties in agreeing the respective responsibilities of local NHS and social care agencies, where both were involved in funding high-level support packages for people with very complex needs, or where funding responsibilities transferred from social services to NHS Continuing Healthcare. For example, one carer wanted to use an underspend in her social care personal budget for a 'special offer' on gym membership for her son, but:

"They said 'No, health's got to pay for that.' I said 'I'm a bit confused now, is that selfdirected?' and they said 'Yes, but that's a health [thing]'" (carer).

Other carers reported difficulties when arrangements for managing the personal health budget were inconsistent with those already in place to manage a social care personal budget; occasionally new arrangements for employing and paying personal assistants were required which worked less well than those they had been using for many years for social care-funded support. It is worth noting that carers recruited to the study were among the first people to be engaged in using a personal health budget and therefore some of these difficulties are likely to reflect the challenges of setting up a new pilot programme. 


\subsubsection{Impact of personal health budgets on the health and quality of life of carers}

One of the main aims of this analysis was to explore whether personal health budgets had an impact on the quality of life and the caring role of carers compared to carers providing help to someone in the control group.

Carers were asked whether specific aspects of their health had been affected by their caring role in the last 12 months. Table 7-7 details their responses. Carers in the personal health budget group generally reported lower instances of having their health affected by their caring role. While the findings are not statistically significant this is nonetheless an interesting observation, particularly given that participants in the personal health budget group had higher levels of need and dependency than participants in the control group (see Chapter 3). However, carers in the personal health budget group reported marginally greater problems with sleep, general feeling of stress and irritableness.

Table 7-7 Caring role and carers' health in the last 12 months

\begin{tabular}{lcc}
\hline $\begin{array}{l}\text { In the last } 12 \text { months has your health been } \\
\text { affected by your caring role in any of the } \\
\text { following ways }\end{array}$ & $\begin{array}{c}\text { PHB Group } \\
\%(\mathbf{N})\end{array}$ & $\begin{array}{c}\text { Control Group } \\
\%(\mathbf{N})\end{array}$ \\
\hline Feeling tired & $77 \%(36)$ & $81 \%(29)$ \\
\hline Feeling depressed & $43 \%(20)$ & $50 \%(18)$ \\
\hline Loss of appetite & $6 \%(3)$ & $14 \%(5)$ \\
\hline Disturbed sleep & $66 \%(31)$ & $61 \%(22)$ \\
\hline General feeling of stress & $62 \%(29)$ & $58 \%(21)$ \\
\hline Physical strain & $43 \%(20)$ & $50 \%(18)$ \\
\hline Short tempered/irritable & $43 \%(20)$ & $42 \%(15)$ \\
\hline Had to see my own GP & $30 \%(14)$ & $28 \%(10)$ \\
\hline Developed health condition & $11 \%(5)$ & $25 \%(9)$ \\
\hline Made existing condition worse & $17 \%(8)$ & $25 \%(9)$ \\
\hline Other & $4 \%(2)$ & $6 \%(2)$ \\
\hline None of these & $11 \%(5)$ & $11 \%(4)$ \\
\hline
\end{tabular}

\subsubsection{Outcomes of personal health budgets for carers}

Overall, carers providing assistance to an individual in the personal health budget group were more likely to report better quality of life and perceived health than carers providing assistance to an individual in the control group. Carers' perceived quality of life (a seven-point scale); perceived health (a five-point scale); health-related quality of life (EQ-5D) and perceived health compared to 12 months ago are shown in Table 7-8. EQ-5D scores are marginally higher (indicating better health) for carers in the personal health budget group than the control group, although the difference is not statistically significant. Looking at health compared to 12 months ago, we can see that $17 \%(n=14)$ of carers in the personal health budget group stated that they felt 'better' compared with $5 \%(n=3)$ of carers in the control group. It is also the case that more carers in the control group $(30 \%, n=17)$ said that they felt 'worse' compared to carers in the personal health budget group ( $24 \%, n=20)$. Quality of 
life and self-perceived health both indicate that those in the personal health budget group have a more positive view.

Table 7-8 Quality of life and well-being for carers

\begin{tabular}{|c|c|c|}
\hline & $\begin{array}{c}\text { PHB Group } \\
\%(\mathbf{N})\end{array}$ & $\begin{array}{c}\text { Control Group } \\
\%(\mathrm{~N})\end{array}$ \\
\hline \multicolumn{3}{|l|}{ Quality of life } \\
\hline So good, it could not be better & $1 \%(1)$ & $2 \%(1)$ \\
\hline Very good & $13 \%(11)$ & $9 \%(5)$ \\
\hline Good & $30 \%(26)$ & $27 \%(16)$ \\
\hline Alright & $45 \%(39)$ & $39 \%(23)$ \\
\hline Bad & $10 \%(9)$ & $17 \%(10)$ \\
\hline Very bad & $1 \%(1)$ & $5 \%(1)$ \\
\hline So bad, it could not be worse & $0 \%(0)$ & $2 \%(1)$ \\
\hline \multicolumn{3}{|l|}{ EQ-5D } \\
\hline Mean score $(n)(s d)$ & $0.75(83)(0.17)$ & $0.72(59)(0.19)$ \\
\hline \multicolumn{3}{|l|}{ Self-perceived health } \\
\hline Very good & $17 \%(8)$ & $14 \%(5)$ \\
\hline Good & $43 \%(20)$ & $36 \%(13)$ \\
\hline Fair & $34 \%(16)$ & $36 \%(13)$ \\
\hline Bad & $4 \%(2)$ & $14 \%(5)$ \\
\hline Very bad & $2 \%(1)$ & $0 \%(0)$ \\
\hline \multicolumn{3}{|c|}{ Health compared to 12 months ago } \\
\hline Better & $17 \%(14)$ & $5 \%(3)$ \\
\hline Much the same & $60 \%(51)$ & $65 \%(37)$ \\
\hline Worse & $24 \%(20)$ & $30 \%(17)$ \\
\hline
\end{tabular}

When asked whether the personal health budget had an impact on their quality of life and health, over $40 \%$ of carers reported it had a positive impact: $41 \%$ thought the personal health budget had an impact on their own health, whilst $56 \%(n=42)$ thought that the budget had an impact on their wellbeing. It would also seem that the deployment option had an effect on carers' well-being. Over twothirds of carers $(68 \% ; n=15)$ caring for patients receiving their personal health budget via the direct payment deployment option said that the personal health budget had had a positive impact on their well-being compared to $36 \%(n=10)$ of carers with participants using an alternative deployment option.

A number of carers that took part in the in-depth interviews reported benefits to their own wellbeing when they could see improvement in the quality of life for the person they supported, derived from the latter's personal health budget:

"Anything that's positive in [son's] life is a positive to me.... It's definitely had an impact on my life as well because he's just happier in himself, emotionally... He's not so emotionally needy, which has made my life a lot easier" (carer). 
Further to this, carers thought that one main direct benefit was an increase in the amount of support provided by paid care workers, which relieved some of the carers' own responsibilities for providing hands-on care. Greater flexibility over respite care arrangements, including new opportunities to 'save' some funding in case additional emergency respite was needed, was another direct benefit. For example, a man caring full-time for his wife had started to have an evening out each week because of the respite funded from his wife's personal health budget:

"Now I've got used to it I quite look forward to it .... It really does make a fantastic difference because I'm actually free.... [The break] blows all the cobwebs away and I enjoy myself for four hours and then I come back and we start all over again" (carer).

"It's changed my life actually because now I've got the flexibility of when I want respite... I can save up the hours for when it's a nice day and I can ask [paid care worker] to take [son] out" (carer).

Personal health budgets allow people to use a wide range of support and services to best meet their own needs. As shown in the previous chapter this did not increase costs in the personal health budget group (and in some cases resulted in a decrease in costs), therefore it is possible that outcomes such as those outlined above were achieved by people restructuring or changing the way they utilise services as opposed to adding services to their original care package.

Indirect benefits enjoyed by carers included new opportunities for an adult child to start going out and engaging in social activities with a paid carer, rather than a parent. Carers saw this as an important first step towards establishing alternative care arrangements when they were no longer able to provide care themselves:

"I like the fact that someone young is coming in and spending a bit of time with [son] so that's already a positive" (carer).

In contrast to some of the findings above, two of the 13 carers that were supporting relatives with stroke or COPD reported no benefits whatsoever for themselves, as their respective partners' personal health budgets were restricted to items directly linked to their healthcare - for example, to fund standard post-stroke rehabilitation services.

Table 7-9 that shows there was a positive trend in almost all domains within ASCOT quality of life for carers (apart from 'time to look after self' and 'occupation and employment'). Similarly, a higher proportion of carers in the control group reported having 'high needs' in all domains barring safety (which is equal (zero) for both groups). Again, this is potentially of interest given that the cared-for participants in the personal health budget group reported higher levels of need at baseline. 


\begin{tabular}{|c|c|c|}
\hline & $\begin{array}{c}\text { PHB Group } \\
\%(\mathrm{~N})\end{array}$ & $\begin{array}{c}\text { Control Group } \\
\%(\mathrm{~N})\end{array}$ \\
\hline \multicolumn{3}{|c|}{ Encouragement / support in caring role } \\
\hline No needs & $46 \%(40)$ & $40 \%(23)$ \\
\hline Low needs & $44 \%(38)$ & $40 \%(23)$ \\
\hline High needs & $10 \%(9)$ & $19 \%(13)$ \\
\hline \multicolumn{3}{|c|}{ Social participation and involvement } \\
\hline No needs & $31 \%(27)$ & $26 \%(15)$ \\
\hline Low needs & $56 \%(49)$ & $53 \%(30)$ \\
\hline High needs & $13 \%(11)$ & $21 \%(12)$ \\
\hline \multicolumn{3}{|c|}{ Control over daily life } \\
\hline No needs & $23 \%(20)$ & $17 \%(10)$ \\
\hline Low needs & $63 \%(55)$ & $67 \%(39)$ \\
\hline High needs & $14 \%(12)$ & $16 \%(9)$ \\
\hline \multicolumn{3}{|c|}{ Space and time for self } \\
\hline No needs & $20 \%(17)$ & $17 \%(10)$ \\
\hline Low needs & $64 \%(56)$ & $61 \%(36)$ \\
\hline High needs & $16 \%(14)$ & $22 \%(13)$ \\
\hline \multicolumn{3}{|l|}{ Safety } \\
\hline No needs & $92 \%(80)$ & $91 \%(53)$ \\
\hline Low needs & $8 \%(7)$ & $9 \%(5)$ \\
\hline High needs & $0 \%(0)$ & $0 \%(0)$ \\
\hline \multicolumn{3}{|c|}{ Time to look after yourself (sleeping / eating) } \\
\hline No needs & $52 \%(45)$ & $59 \%(34)$ \\
\hline Low needs & $31 \%(27)$ & $22 \%(13)$ \\
\hline High needs & $17 \%(15)$ & $19 \%(11)$ \\
\hline \multicolumn{3}{|c|}{ Occupation and employment } \\
\hline No needs & $12 \%(10)$ & $16 \%(9)$ \\
\hline Low needs & $79 \%(68)$ & $71 \%(41)$ \\
\hline High needs & $9 \%(8)$ & $14 \%(8)$ \\
\hline \multicolumn{3}{|c|}{ Skills needed for caring } \\
\hline No needs & $92 \%(79)$ & $91 \%(50)$ \\
\hline Low needs & $7 \%(6)$ & $7 \%(4)$ \\
\hline High needs & $1 \%(1)$ & $2 \%(1)$ \\
\hline
\end{tabular}

\subsection{Discussion}

The main findings suggest personal health budgets did not have an impact on the change of informal care receipt at follow-up compared to conventional service delivery once the confounding variables were accounted for in the difference-in-difference multivariate analysis.

In the whole sample analysis we did not find a significant difference in the reported use of informal care between the personal health budget and control group. There were some significant results in the sub-sample analysis in this regard. Personal health budgets of model 3 type were associated with 
an increase in informal care receipt inside the household relative to controls. Personal health budgets of model 1 type were associated with a decrease in informal care receipt from outside the household relative to controls. We also found that personal health budgets of model 2 type were associated with an increase in informal care receipt inside the household relative to controls. This pattern of results does not entirely accord with our prior expectation that personal health budgets would reduce the need for people to rely on informal care.

Carers providing assistance to an individual in the personal health budget group were more likely to report better quality of life and perceived health compared to carers assisting an individual in the control group. Carers also seemed to be satisfied with the personal health budget process in terms of support planning, the amount of the budget and the amount of help that was offered when deciding what services or support to purchase from the personal health budget. However, we need to exercise caution here due to the small sample sizes for the sub-group analysis.

Findings from the semi-structured interviews (Davidson et al., 2012) suggested that carers derived little direct benefit from budgets that were tightly targeted at the symptoms and healthcare of the person they supported. The potential for benefit appeared to be considerable among those carers providing substantial amounts of support to a relative with a progressive long-term neurological condition or someone eligible for NHS Continuing Healthcare. As with the study of the impact of social care individual budgets on carers (Glendinning et al., 2009), these benefits could be realised if carers' needs for regular breaks, or for flexibility over the provision of respite care, were taken into account in the care/support plan. Carers may also experience indirect benefits if they see improvements in the health, well-being, or quality of life of the person they support. However, difficulties in agreeing complex funding responsibilities or alterations to established arrangements for employing paid carers could potentially jeopardise and undermine these benefits.

Overall the findings suggest that personal health budgets have the potential to have a positive impact on the lives of carers. However, the findings should be treated with caution due to the small sample of carers, and a larger research study would be required before firm conclusions could be made. 


\section{Personal health budgets - successes and challenges}

\subsection{Summary}

This chapter draws together the main evidence to discuss the operation, benefits and costs of personal health budgets. We then outline the limitations of this highly complex evaluation that should serve as cautions when interpreting the results.

Overall the chapter outlines how the findings point to the conclusion that personal health budgets have benefits in terms of higher-order aspects of quality of life that are valued by people beyond improvements in health status per se.

The value society places on the improvement of quality of life, particularly through improvements in control and autonomy, will be critical in the overall conclusion about the cost-effectiveness of personal health budgets. In the main analysis we found personal health budgets to be cost effective when the willingness to pay for a unit improvement of ASCOT quality of life over a year is more than $f 30,000$.

The research limitations are broadly divided into three categories:

Evaluation design

- Rather than a single intervention there was a whole range of implementations of personal health budgets with different models operating in the 20 in-depth pilot sites over six patient groups. Personal health budgets were entirely new and a radical departure in some areas, so the operation of PHBs was developing and changing during the course of the evaluation.

- The pragmatic controlled design we used involved both randomised and non-randomised subsamples. In choosing the study design, the potential for selection bias in the absence of randomisation was balanced against the potential for contamination bias, that is, the control group being affected by the operation of the pilot programme. In the study, we had some significant differences between the groups at baseline. The control group was significantly older but also healthier than the personal health budget group. We have accounted for any potentially biasing consequences by using a difference-in-difference design and also using baseline control factors in the analysis where required.

Loss to follow-up

- We expected drop-out rates to be higher for this study population as a result of much lower than population-average health status and well-being scores. We have suggested that the reasons for drop-out are due to baseline factors to a significant extent and therefore not a source of bias in the evaluation of the personal health budget pilot programme.

Data quality

- A number of assumptions had to be made to produce like-with-like cost estimates between personal health budget and control groups. 


\subsection{Introduction}

Personal health budgets represent a significant departure from the conventional operation of the health service. There has been considerable debate about the potential advantages and disadvantages of personal health budgets. This evaluation has aimed to shed some light on these questions. In this chapter we gather our analysis results and evidence together to discuss the operation, benefits and costs of personal health budgets. In the next chapter we build on our findings to discuss the implications for rolling out personal health budgets nationally.

We begin by assessing, overall, the effectiveness of personal health budgets, their costs and their cost-effectiveness. We then turn to a discussion of the robustness of the findings in terms of the strengths and limitations of the evaluation.

\subsection{Costs and benefits}

We used a range of measures of outcome or effectiveness in the evaluation to build up a comprehensive picture of the potential impact of personal health budgets. Broadly speaking, we can distinguish our more objective clinical measures, such as the $\mathrm{HbA1c}$ blood glucose test, lung-function and mortality rates, from the more subjective measures that involve self-report about health and social care related quality of life (EQ-5D and ASCOT), psychological well-being (GHQ-12) and subjective global well-being. Of the latter, there is also the distinction between measures focused on personal health-related functioning, such as EQ-5D, and those which emphasise the achievement or otherwise, through all means, of activities and experiences that are important to care-related quality of life, such as ASCOT. Finally, subjective (global) well-being measures seek people's overall rating of life satisfaction, worry, happiness and satisfaction with health.

Key results with respect to the outcomes measures were as follows.

- The results of the analysis indicate that personal health budgets overall have a (statistically significant) positive impact on care-related quality of life (ASCOT) and psychological wellbeing (GHQ-12).

- We found that the size and significance of the effect of personal health budgets on these measures differed significantly for different configuration models of personal health budgets. Personal health budgets models with high degrees of flexibility and choice showed especially strong effects. The data also suggested that budgets of more than $£ 1,000$ per year ( $40 \%$ of all personal health budgets) were more effective than those with less than $£ 1,000$ in the budget. This latter result is likely to reflect the more limited scope of personal health budgets with small budgets to make a meaningful difference. It might also be due to people with higher levels of need - and hence larger budgets, other things equal - having a greater capacity to benefit from personal health budgets than people with lower needs.

- The difference in effectiveness of personal health budgets for different health conditions was less marked than we anticipated. With the exception of COPD, we found no significant differences in this regard. COPD patients showed significant improvements on ASCOT, GHQ12 and subjective well-being. 
- Personal health budgets appeared to have no impact on health status per se. We found no significant effects with regard to the two clinical measures, $\mathrm{HbA1c}$ blood glucose test and lung-function, as used for the diabetes and COPD sub-groups. Furthermore we found no significant difference in mortality rates between the groups.

- Consistent with the previous result, the study did not find that personal health budgets had a significant effect on EQ-5D compared to the control group.

The cost analysis included a wide range of service and support expenditures, including those classified as social care ${ }^{42}$, nursing, therapy and well-being services that can be secured (directly) using a personal health budget; and primary and secondary health care services that might be affected indirectly. As regard the cost of services and support, personal health budgets can be provided inaddition to conventional services or as substitutes for conventional services. The main findings with regard to costs were as follows.

- Personal health budgets were largely cost neutral (although there was substantial variation in the level and types of costs between participants). For certain categories of expenditure, the personal health budget group had slightly lower costs than the control group after correcting for baseline differences. In particular, indirect costs (primary and secondary care costs) were lower for the personal health budget group after subtracting baseline cost differences.

- This neutral result was found where we used the relatively conservative assumption that many personal health budgets $(60 \%+)$ were provided in-addition to conventional services. For high-budget personal health budgets (more than $f 1000$ a year) where the PHB was more likely to be provided as a substitute for conventional service delivery, costs were lower after baseline for the personal health budget group compared to controls at the $10 \%$ significance level.

The analysis focused on recurrent costs and did not include one-off costs such as set-up costs. We did not attempt a comparison of the transaction costs of personal health budgets compared to conventional service delivery arrangements due to the variation with implementing the initiative within the pilot sites.

The quantitative analysis also considered the impact of personal health budgets on the utilisation of carers. We did not, however, find an increase in the proportion of budget holders claiming to have either co-resident or other carers, as compared to the control group.

The cost-effectiveness analysis assessed differences in net monetary benefit between the personal health budget and control groups. Net monetary benefit (NMB) is the $f$-equivalent value of gains in

\footnotetext{
${ }^{42}$ Social care-related services that meet both identified health and social care needs.
} 
EQ-5D or ASCOT (initially assumed to be $\mathrm{f30,000}$ per unit gain in EQ-5D or ASCOT for one year) less the cost of services and support.

- For all types of personal health budgets, the results suggested that personal health budgets were cost-effective with regard to ASCOT outcomes: people in the personal health budget group reported higher net (monetary) benefit than for people in the control group after subtracting any baseline difference in net benefit between the groups. This result was significant overall at the $10 \%$ significance level.

- We did not, however, find a statistically significant difference in net benefit between the groups when benefits were measured on the EQ-5D scale.

- When we compared specific types of personal health budget to conventional service delivery we found cost-effectiveness at a higher level of significance. People using personal health budgets implemented as model 1 reported a greater positive change in ASCOT compared to those in the control group, significant at the $5 \%$ level. The same result also occurred for people using personal health budgets with a budget of more than $£ 1000$ a year, also with significance at $5 \%$.

- We found little difference in the effect size of personal health budgets for the different health condition sub-groups compared to the whole-sample effect. We did find personal health budgets to be significantly cost-effective (at 10\%) when just comparing personal health budget and control groups in the sub-samples for patients within the NHS Continuing Healthcare and mental health cohorts. These results suggest, somewhat tentatively, that the net benefits of a personal health budget are greater for NHS Continuing Healthcare and patients with mental health problems than for other patient groups.

- Further investigation of the main effect routes estimated that people with personal health budgets would have significantly better ASCOT outcomes than controls after removing any differences in total expenditure on services. The same result was also found for EQ-5D when only $f 1,000+$ personal health budgets were considered. These findings support our conjecture that the extra choice and control, and its consequences, are the main reasons why personal health budgets produce greater net benefits than conventional service delivery (rather than just differences in resourcing levels for personal health budgets). Choice and control can be valued for its own sake and as a means for people to secure services and support that better fits with their own needs and preferences.

The findings point to the conclusion that personal health budgets have benefits in terms of higherorder aspects of quality of life that are valued by people beyond improvements in health status per se. There is a substantial literature on defining, identifying and measuring the consequences that people experience as a result of using health, social care and other related public services. Much of this literature recognises that these consequences are more far-reaching than improvements in health alone, at least where 'health' is understood in the sense of people being free of impairing conditions, be they physical or mental (Sen, 1982; Sen, 1993; Grewal et al., 2006; Higgs et al., 2003; Schalock, 2004).

Two inter-related ideas are particularly relevant: first, a distinction between more basic human needs and higher-order needs (Maslow, 1968; Nussbaum, 1995; Nussbaum and Sen, 1993); and second, 
that it is the meeting of needs which is important, rather than how they are met. These ideas imply that having good health and also having control or autonomy (economic, political, or otherwise) are capabilities with which to achieve happiness, well-being and good quality of life. Arguably, the results suggest that personal health budgets serve to improve capability in the latter sense.

The value society places on the improvement of quality of life, particularly through improvements in control and autonomy, will be critical in the overall conclusion about the cost-effectiveness of personal health budgets. In the main analysis we found personal health budgets to be cost-effective when the willingness to pay for a unit improvement of ASCOT quality of life over a year is more than f30,000. ${ }^{43}$

These benefits are expressed in monetary terms when a willingness to pay threshold is applied to the underlying scale. Measures like ASCOT or EQ-5D are amenable scales for this purpose because they are designed to produce quantitative ratings of experiences that people have which are relevant to their quality of life - for example, being in pain, being able to conduct usual activities, feeling in control of their lives etc. People's valuation of difference in quality of life are made with reference to the trade-offs they are willing to make in terms of living with better quality of life but dying earlier. They are therefore 'anchored' in a meaningful way.

\subsection{Limitations}

The evaluation of the personal health budget pilot programme was highly complex. As such there were a number of limitations that should serve as cautions when interpreting the results.

\subsubsection{Evaluation design}

Rather than a single intervention there was a whole range of implementations of personal health budgets with different models operating in the 20 in-depth pilot sites over six patient groups. Personal health budgets were entirely new and a radical departure in some areas, so that the operation of personal health budgets was developing and changing during the course of the evaluation. Other sites were further ahead and made more progress than others. This did however mean that the establishment of detailed and consistent evaluation protocols and designing study selection criteria in advance was not always possible. A particular difficulty was in establishing what the personal health budget was for and which services could be purchased or secured with it before the evaluation started.

As a 'process/system' intervention there was no way to make the evaluation 'blind' in the sense that participants and others would not know whether they were in the personal health budget or control

\footnotetext{
${ }^{43}$ Whilst there is no well-established willingness-to-pay threshold for ASCOT, because its scaling is comparable to the EQ-5D scale (and anchored in the same way), we can follow NICE guidelines in using a willingness-to-pay threshold of $£ 30,000$ for ASCOT as it is used for EQ-5D.
} 
groups. It was possible in some cases to randomly assign people to the personal health budget and control groups but only where sites were set up so that personal health budgets and conventional service delivery options were available to the same health practitioner. The majority of sites, by contrast, set up personal health budgets in particular areas, a configuration that created difficulties in drawing control group participants from the same area. For these reasons a standard RCT design was both not feasible and inappropriate.

The pragmatic controlled design we used involved both randomised ( $26 \%$ of participants) and nonrandomised sub-samples (74\%). In the latter, the control group participants were drawn from different localities than participants in the personal health budget group. In choosing the study design, the potential for selection bias in the absence of randomisation was balanced against the potential for contamination bias ${ }^{44}$ when randomisation is used at an individual level. In the study, we had some significant differences between the groups at baseline. The control group was significantly older but also healthier than the personal health budget group. We have accounted for any potentially biasing consequences by using a difference-in-difference design and also using baseline control factors in the analysis where required.

\subsubsection{Loss to follow-up}

Another tension in the design of the evaluation was between allowing sufficient elapsed time after baseline for the effects of personal health budgets to be felt on the one hand, and minimising loss to follow-up on the other. The experience from the evaluation of the individual budgets pilots (Glendinning et al., 2008) was that a six-month follow-up period was unlikely to be sufficient and so we opted for a main follow-up period of one year. As a consequence, although final recruitment rates were good, drop-out rates were an issue. Some 2,393 people entered the study at baseline. ${ }^{45}$ Of these, 158 people died before follow-up, leaving 2,235 cases as the active sample. We had returned main follow-up instruments from 1,524 cases or $68 \%$ of the active sample. Regarding any outcomes instrument data, we had at least some follow-up data in 1,656 cases ( $74.1 \%$ of the active sample) - see Appendix $\mathrm{C}$ for more details.

The study population was also in the most part very frail with much lower than population-average health status and well-being scores. We expect drop-out rates to be higher for this study population as a result, but can argue that the reasons for drop-out are due to baseline factors to a significant extent and therefore not a source of bias in the evaluation of personal health budgets.

\footnotetext{
${ }^{44}$ that is, the control group being affected by the operation of the PHB pilot.

${ }^{45}$ Consent forms for the study were gained from 2,700 people but 302 people were excluded from the study: because they had neither taken part in the baseline or follow-up interviews and had in effect withdrawn consent before baseline; because they were in residential care at baseline; because they were under 18; or because they had died before baseline.
} 
Multiple-imputation is a now-established method to deal with drop-out and missing data. The study used (a) a wide range of predictors in the statistical models to estimate values for missing data, and (b) used different specifications of the imputation model to assess the sensitivity of the results to the implicit assumptions of those models.

\subsubsection{Data quality}

The quality of the outcomes data was good, especially the main subjective instruments. Because we had to rely on local site tracking and records regarding mortality data, we were less able to rate the quality of this data. Service data was drawn from a number of sources. Where possible we did not rely on self-reported use, instead interrogating care plans, using medical records and hospital episode statistics. Some data had to be collected by self-report however. Another issue was the sheer range of services and support people in the study could have used. As outlined in Chapters 2 and 6, a number of assumptions had to be made to produce like-with-like cost estimates between personal health budget and control groups.

\subsubsection{Sensitivity}

A number of assumptions had to be made in the analysis. We tested the sensitivity of three types of assumption:

- Statistical assumptions. For key analyses such as the cost-effectiveness estimates we used both parametric and non-parametric (bootstrapping) methods. We found very little difference in the results.

- Costing assumption. A key assumption in this regard was the identification of personal health budgets provided in-addition or as a substitute for conventional services. In the main analysis we adopted conservative assumptions (i.e. in favour of conventional service delivery) in this regard. On testing the sensitivity of the main results to this assumption, we did not find any qualitative impact on the results until quite unrealistic assumptions were tried.

- Multiple imputation. To test the sensitivity of the main results we, first, added a further five imputations to our main dataset with a different randomly selected seed value, and second, used a variant imputation model. The main results in both alternative cases were very similar to the original estimates, with, if anything, slightly better statistical significance. In particular, with both the alternative dataset and the alternative imputation model the results for the whole sample analysis indicated that personal health budgets were cost-effective on the ASCOT scale at the $5 \%$ significance level rather than at $10 \%$. 


\section{Implementing personal health budgets}

\subsection{Summary}

This chapter draws together the evidence and provides a series of policy recommendations:

- The optimal use and design of personal health budgets.

- This section will draw mainly on results of the comparative evaluation and focus on which options were most cost-effective.

- The configuration of personal health budgets and supporting processes from the individuals' perspective.

- The qualitative analysis results mostly inform the recommendations made in this section.

- The initial set up and configuration of personal health budgets from an organisational perspective.

- The wider impact of personal health budgets, assessing implications of personal health budget use for health and social care integration, commissioning and diversification of supply in the health and care economy.

The key recommendations are:

- Personal health budgets are best offered to people with greater need, to act as a substitute for conventional service delivery.

- Personal health budgets are cost-effective for people with mental health problems and those receiving NHS Continuing Healthcare but are inconclusive for other health conditions. Focusing initial roll-out on these two groups is suggested, but the study cannot recommend specific direction for the other health condition cohorts.

- Policy makers should anticipate that the use of personal health budgets is likely to result in a higher level of expenditure going to 'non-conventional' (i.e. non-NHS) providers. Further research is required to better understand the scale of these changes.

\subsection{Introduction}

The evaluation aimed: first, to investigate the operation, effectiveness and cost-effectiveness of personal health budgets; and, second, to inform the national roll-out of personal health budgets by providing information about how the initiative should be implemented. Clearly, these are interrelated aims: the implications for the roll-out involve identifying which kinds of personal health budget, in what circumstances and for whom, have the greatest effect. We would recommend rollout to be arranged on this basis.

During the evaluation period there was a growing emphasis on informing the roll-out of personal health budgets although accepting that decisions would be subject to the research findings regarding the costs and benefits of personal health budgets. On 25 September 2012, the Government announced that $f 1.5$ million will become available to support the roll-out of the initiative beyond the 
pilot programme. On 4 October 2011, the Secretary of State for Health announced that, subject to the evaluation, by April 2014 everyone in receipt of NHS Continuing Healthcare (NHS CHC) will have the right to ask for a personal health budget, including a direct payment. Furthermore, it is planned that the new Clinical Commissioning Group (CCGs) will be able to offer personal health budgets on a voluntary basis more widely.

This chapter aims to present our findings and evidence to inform the question of how best to direct a roll-out. The implications of the study will be organised into the following four sections:

1. The optimal use and design of personal health budgets.

- This section will draw mainly on results of the comparative evaluation and focus on which options were most cost-effective.

2. The configuration of personal health budgets and supporting processes from the individual's perspective.

- The qualitative analysis results mostly inform the recommendations made in this section.

3. The initial set up and configuration of personal health budgets from an organisational perspective.

4. The wider impact of personal health budgets, assessing implications of personal health budget use for health and social care integration, commissioning and diversification of supply in the health and care economy.

Our analysis in the section will consider cross-cutting themes of how to set up and facilitate the implementation of personal health budgets, and which models appear to work best and for which groups, including design choices about a personal health budget system, such as care planning, deployment options and resource allocation systems.

\subsection{Optimal use and design of personal health budgets}

\subsubsection{Should personal health budgets be deployed?}

This evaluation took a sample of people with chronic obstructive pulmonary disease, diabetes and long-term neurological conditions, mental health, stroke, or those using NHS Continuing Healthcare in 20 localities to assess whether personal health budgets are cost-effective. The study suggested that personal health budgets are cost-effective, producing valued well-being benefits with a largely neutral impact on (recurrent) costs and on health/clinical outcomes.

This conclusion applies to the population of patients from which this sample was drawn, in terms of an average effect. Clearly, the net benefits of personal health budgets vary between individuals, and therefore we would expect to see variation between health condition sub-groups and between localities. But this 'whole-sample' conclusion does not rest on these distinctions. We had the benefit, in this case, of a larger sample size - and therefore more precision for the result - but with a loss of specificity. The sub-group analyses, by contrast, gave greater specificity, but at the price of lesser statistical power. 
The overall implication is that personal health budgets are cost-effective for the study population, given the assumptions we have made, and should be rolled out for this population if these assumptions are acceptable. The main assumptions concern: the monetary value placed on carerelated quality of life (as measured by ASCOT) ${ }^{46}$ assessing cost implications in terms of recurrent health and social care service use ${ }^{47}$ and the level of statistical significance. ${ }^{48}$ In as far as the localities in the sample are representative of the whole country, on average, the results support a national roll-out where people are offered a personal health budget.

\section{Recommendation: Overall, the study found that personal health budgets were cost-effective, given the assumptions that were made (e.g. regarding the value of ASCOT quality of life), and should be rolled out for the study population (if these assumptions are acceptable).}

In practice, personal health budgets will be more cost-effective for some sub-groups and less costeffective for others. We were able to explore some of these differences in the sub-group analysis, the results of which we turn to next. Nonetheless, if a more inclusive roll-out programme is adopted, then ideally there would be an opportunity to review the cost-effectiveness of personal health budgets and on that basis refine how the offered use personal health budgets is targeted for different health condition groups.

In contrast to the cost-effectiveness results using ASCOT-measured quality of life, the analysis did not find that personal health budgets had a different effect on the size of participants' net benefit (compared to the control group) when those benefits were measured using the EQ-5D health-related quality of life scale or using clinical markers. We might therefore ask how relevant therefore is the ASCOT-measured quality of life result? People value care-related quality of life as measured using the ASCOT scale in that they are willing to exchange shorter life expectancy (with full ASCOT-measured quality of life) to avoid a longer life expectancy with poorer ASCOT-measured quality of life. We also sought to understand whether the sensitivity of the ASCOT measure was concentrated among those people using social care. We found that whether or not the participant was a social care service user at baseline made no difference to the size of the improvement in ASCOT-measured quality of life associated with personal health budget use (relative to the controls)..$^{49}$ If ASCOT was only sensitive to

\footnotetext{
${ }^{46}$ i.e. $£ 30,000$ or more per ASCOT QALY.

${ }^{47}$ i.e. putting aside any extra transaction costs that might accrue in using personal health budgets .

${ }^{48}$ In this case a 90\% confidence level (although the sensitivity analysis suggests this is a conservative assumption).

${ }^{49}$ This assessment was made by estimating whether the impact of personal health budgets on ASCOT-measured quality of life at follow-up (relative to the control group and controlling for baseline differences) was different in size comparing social care users at baseline with non-social care users. The interaction term for baseline social care use was insignificant $(p=$ 0.992).
} 
improvements in quality of life stemming from social care use only, we would have expected a significant difference in this effect size..$^{50}$

\subsubsection{Refining the implications for targeting of personal health budgets}

The study sample was drawn from a range of people with long-term conditions and this allowed us to conduct some sub-sample analyses to assess the costs and benefits of personal health budgets for different sub-groups. The sample could have been divided up in a number of ways, but we focused on sub-samples where (a) we expected that personal health budgets might have differential effects and (b) where there was sufficient sample size in the respective sub-group.

The relevance of these sub-sample analyses for policy about the national roll-out of personal health budgets is that they provide some insight into which groups of patients show better than average, and which worse than average, improvements in net benefits from the use of personal health budgets relative to conventional methods. Similarly, we can assess whether certain types of personal health budget process show better or worse than average effects. This information can help in targeting personal health budgets to their most cost-effective use.

We assessed these effects for the following sub-groups:

- Personal health budget process models;

- Size of personal health budget;

- Socio-demographic factors;

o Health conditions.

\subsubsection{Personal health budget process models}

As outlined in Chapter 1, a range of implementation models were used to implement personal health budgets, varying according to: whether the budget level was known before the care and support planning process began; what flexibility there was in terms of services/support that could be purchased with the budget; and the choice of deployment options (including DPs). Chapters 5 and 6 reported analyses suggesting these models had quite different impacts on improvements in outcome measures or effectiveness, and on the cost-effectiveness of personal health budgets.

Implementation models 4 and 5 had a positive impact in terms of well-being outcomes, but model 3 generally had a negative impact. Model 5 offered pre-determined budget levels, flexibility and deployment choice. Model 4 offered only the flexibility and choice component. Pilot sites implementing personal health budgets following model 3 limited the degree of flexibility and choice

\footnotetext{
${ }^{50}$ This result is not definitive in this respect because other factors change after baseline - but it does support our expectation that ASCOT is not just sensitive to the impact of social care services but also other forms of support for people with long-term conditions.
} 
around services and deployment. Together, the findings suggest that while knowing the budget is a fundamental part of the personal health budget initiative, flexibility and choice given to individuals and families are perhaps of greater importance.

In the whole-sample analysis we did not find a significant difference in the reported use of informal care between personal health budget and control groups. There were some significant results in the sub-sample analysis in this regard. Personal health budgets of model 3 type were associated with an increase in informal care receipt inside the household relative to controls. Personal health budgets of model 1 type were associated with a decrease in informal care receipt from outside the household relative to controls. We also found that personal health budgets of model 2 type were associated with an increase in informal care receipt inside the household relative to controls. This pattern of results does not entirely accord with our prior expectation that personal health budgets would reduce the need for people to rely on informal care.

Personal health budgets produced the most significant improvements in net benefit compared to controls (significant at the $5 \%$ level for the ASCOT scale) when implemented in model 1 form. By contrast, personal health budgets in model 3 form were not cost-effective compared to conventional practice in the control group.

Although somewhat intuitive, the implication for the roll-out is:

Recommendation: That personal health budgets should be configured to give recipients choice and flexibility over how the budget can be used.

In this respect, new personal health budget sites should resist the inclination to put in place limits in this regard.

\subsubsection{Size of the budget}

The monetary size of personal health budgets in the study varied enormously. Those of modest scale were generally being provided on top of conventional service delivery, not as substitutes. Our prior expectation was that if personal health budgets were going to offer improved net benefits then this improvement would be proportional to the size of the budget. Compared to modest size budgets, those of larger (monetary) size might not only indicate that the recipient had higher levels of service need - and therefor a greater capacity to benefit from services - but also that a greater proportion of their service and support needs are being met from the budget rather than via conventional service delivery.

We assumed in the analysis that high monetary amounts indicate that the personal health budget was funded by substituting for existing services, while the smaller budgets were in addition to what the individual would have received conventionally.

The results showed that larger monetary value budgets had a significantly different impact on both costs and cost-effectiveness than smaller personal health budgets. Overall, the use of a high-value personal health budget (i.e. greater than $£ 1000$ per year) was associated with significantly lower service costs at follow-up compared to the control group (accounting for baseline differences). 
Furthermore, people in the high-value personal health budget group had significantly higher net benefits than those people in the control group (accounting for baseline differences) - at the $95 \%$ confidence level for ASCOT-measured benefits and the $90 \%$ confidence level for EQ-5D-measured benefits. By contrast, lower value budgets did not show significant differences in net benefits accrued compared to controls.

The monetary size of personal health budgets in the sample varied significantly between different health conditions and other factors, clouding our interpretation of these results. Nonetheless, the findings would support a policy of offering personal health budgets to people with greater need to substitute for conventional services.

Recommendation: Personal health budgets are best offered to people with greater need, to act as a substitute for conventional service delivery.

This does not rule out offering more modest size personal health budgets in other circumstances, but only with the expectation that the net benefit of recipients is not likely to be greater than the net benefits they get from conventional service delivery.

\subsubsection{Health conditions}

The study sample drew participants from a population of people with different primary health conditions (and, indeed, multiple conditions). This provided the opportunity to explore whether personal health budgets are more beneficial for some groups rather than others. The study found that personal health budgets were cost effective (i.e. averaged higher net benefit) compared to the control groups for both the mental health and NHS Continuing Healthcare groups, at the $90 \%$ confidence level. ${ }^{51}$ We did not find significant differences for the other health condition groups.

The small sample sizes for the sub-group analyses mean the results for the other health conditions are generally non-conclusive on their own. We cannot be confident that a non-significant result implies that there was no personal health budget effect for the health condition group in question; rather, it might be that we did not have the statistical power to be able to confidently reject the possibility that no effect exists. Limits on the scale of the study meant that we could only realistically expect to detect strong personal health budget effects for individual sub-samples.

In terms of the implications for roll-out, the results do suggest - accepting the usual assumptions that efforts should initially be focused on offering personal health budgets to people receiving NHS Continuing Healthcare and to those with mental health problems as their primary health condition. The latter result is in line with the findings from the evaluation of individual budgets in social care. We cannot make specific recommendations for the other health condition groups for the reasons outlined, and need to defer to our overall findings in this case.

\footnotetext{
${ }^{51}$ Again, on the basis of ASCOT-measured net benefit.
} 
Recommendation: The results indicate that personal health budgets are cost-effective for people with mental health problems and those receiving NHS Continuing healthcare but are inconclusive for other health conditions. Focusing initial roll-out on these two groups is suggested, but the study cannot make specific recommendations for the other health condition cohorts.

\subsubsection{Socio-demographic factors}

Many particular socio-economic characteristics of individuals could make them more or less predisposed to benefiting from personal health budgets. This study focused on three: age, gender and income levels. As regards the former, older age is generally associated with higher levels of need and support and therefore a greater opportunity for personal health budgets to result in changes in services. The individual budgets evaluation, however, suggested that older people were less likely to benefit from social care personal budgets. In this study we distinguished between people aged 75 and over and those younger than 75 .

The analysis found that younger people with personal health budgets had higher ASCOT outcome scores at follow-up than younger people in the control group (after accounting for baseline differences in ASCOT scores between the two groups). There was no significant difference in outcome scores for people over 75 between the groups. We did not find significant differences by age using EQ-5D-measured outcomes.

By contrast, when we investigated the impact of personal health budgets on net benefit for different age groups, we found that personal health budgets were cost-effective for people over 75 at the $90 \%$ confidence level, but that there was no significant differences between net benefit levels for people aged younger than 75 with personal health budgets compared to people aged younger than 75 in the control group. ${ }^{52}$ This apparent inconsistency was due to the greater cost savings that were indicated for over $75 \mathrm{~s}$ using personal health budgets compared to younger people. This result is consistent with our contention that people over 75 used a higher level of services and so there was more opportunity to make savings with personal health budgets.

The complex nature of this analysis with regard to age makes it difficult to draw out clear implications. We need to be particularly aware of the relatively large variation in the cost data and note that the sensitivity analysis suggested some volatility of these findings based on the assumptions made. Even putting aside issues around the legality of targeting policies on the basis of age, these results are certainly not unambiguous in this respect anyway.

The analysis did not find any differential impact of personal budgets in terms of ethnicity, gender or income (using uptake of income benefits as a proxy for income levels). Note that sample sizes were

\footnotetext{
${ }^{52}$ A significant difference was found for under $75 \mathrm{~s}$ in the sensitivity analysis (PMM results).
} 
small for the ethnicity sub-group analyses; a non-significant result might arise from an underpowered sample size.

Recommendation: the results do not suggest that the impact of personal health budgets is differentiated by ethnicity, gender or income of recipients - no particular targeting in this respect need therefore be considered.

\subsection{Configuring personal health budgets from the individuals' perspective}

The in-depth interviews with budget holders and carers highlighted a number of issues that could provide further guidance for the future roll-out of personal health budgets. It should be kept in mind that the sub-samples of budget holders and carers who took part in the in-depth interviews were among the earliest to be offered a budget within the pilot programme. Moreover, few had begun to receive services, care or equipment funded through the personal health budget by the time of the three-month interviews, and several still did not have a budget in place when re-interviewed at nine months. Consequently, they, and some of their experiences, may not have been typical of the pilots once these were fully operational. Nevertheless, their views provide valuable learning about the factors that contributed to more or less positive experiences of implementation.

\subsubsection{Initial impressions and understanding}

Despite the fact that the participants had been specifically recruited to the pilot programme, initial understandings of personal health budgets were mixed. While some understood the principle of a personal allocation of resources, others thought they were drawing on a general fund rather like a grant-making scheme, so that if they did not use the budget it could be allocated to someone else. Moreover, around half the sub-sample reported initial anxiety about trying a budget, including whether other healthcare treatments would be affected; the size of the budget; how they would decide on its use; whether a desired use would be approved; and the 'hassle' involved.

These concerns highlight the importance of giving clear, detailed information about personal health budgets from the earliest contacts with potential budget holders, and ample subsequent opportunities for discussions with healthcare staff as new questions arise. Participants confirmed that written leaflets could be useful in providing a general overview, but these could be difficult to relate to individual circumstances. Subsequent face-to-face discussions with health professionals were particularly valued, especially if patients had been very unwell and unable to retain detailed information when first told about the budget.

Recommendation: Clear, detailed information about personal health budgets should be given to potential budget holders from the earliest contact.

\subsubsection{The level of the personal health budget}

Three months after the offer of a personal health budget, only a minority of the sub-sample knew how much their budget would be; even at nine months a few participants still did not know the level of their budget. Sometimes this was because they still thought the budget was a centralised 'pot' of money rather than an individual allocation; others thought the equipment or service that had been 
funded through the budget was a one-off grant, in response to a specific expressed need, rather than part of a personal health budget. This meant that most participants started - and sometimes completed - planning the use of their budget without knowing how much it was. They were also therefore unable to assess the adequacy of the budget for their needs, or how much of the budget remained unspent:

"I think it is [enough]; the problem is we don't know how much we've spent" (budget holder).

"I'm worried that there's not going to be enough, come the end of the year" (budget holder).

As reported in Chapter 5, around a quarter of participants thought they knew how their budget had been calculated, but the basis for these calculations varied widely. Some thought it was a standard amount for their particular health condition; others thought it reflected the cost of the items they planned to use the budget for; a third group thought it was related to the costs of the NHS services they used. Most, however, would have been interested in knowing.

Some interviewees had used their own money to pay for health or related services for various reasons: because they were unclear about what the budget was for or how it could be used; because their plans for using the budget had been refused; or because the budget was insufficient to purchase all the services or items they wanted:

"They said 'Well no, we're not going to give you the full amount, we can give you a basic rate and then ... you're going to have to pay a top up for that" (budget holder).

It was clear from the interviews that there was considerable scope for improving the information given to potential budget holders about the indicative level of the budget, especially before starting care/support planning.

\section{Recommendation: Potential budget holders should be given better information about the indicative level of the budget, especially before starting care/support planning.}

\subsubsection{Support and care planning}

Participants emphasised the wish to use their budget 'wisely', and 'in the right way'. Most had some ideas for how they might use their budget at the point of accepting the offer of a personal health budget. However, not all were able to realise these plans; indeed, even at nine months, there was considerable uncertainty among participants about what personal health budgets could and could not legitimately be used for. Participants found it helpful to be given a list of suggestions; information about how other people with similar conditions had used their budget to improve their health and quality of life was particularly useful, as this helped them to 'think outside the box'. However, it was important that such lists were treated as suggestions only, rather than constituting a definitive menu, otherwise participants felt uncomfortable about or inhibited from requesting items they thought would benefit them but that were not on the list. 
It was important for information about possible uses of the budget to be freely available; some participants felt uncomfortable asking if they could use their budget for a particular item or service and consequently used very little of the budget. A few felt they had been pressured by pilot site staff into agreeing a particular use for their budget, although it was not high on their own list of priorities. Occasionally, participants discovered the budget was being used for items they did not recall agreeing to.

Budget holders and carers who had deteriorating long-term neurological conditions or were eligible for NHS Continuing Healthcare usually had little difficulty deciding how to use their budget; in many instances the budget would enable them to maintain or increase the support they received from paid carers or personal assistants. However, one group of participants who had great difficulty with care/support planning were those offered a budget shortly after suffering a stroke, sometimes even before their discharge from hospital:

"When you're not well - and I was still quite confused early on - it's hard to really know what you're going to need and what you want" (budget holder).

Experiences of getting support/care plans approved varied from quick and smooth to complex and lengthy; in the latter cases, frustration was compounded by participants' lack of clarity over who exactly made approval decisions. Common reasons for delays included the need to obtain risk assessments from GPs or hospital consultants; and care plans that included items not on a standard list that had also so far not been approved for anyone else in the local pilot. Delays in obtaining approval led to participants feeling 'saddened', 'upset', 'uncertain' and 'destabilised', particularly if they were keen to start a particular treatment or exercise. A further cause of major distress was when items within a support/care plan were turned down. Participants expressed anger at being unable to use 'their' budget for things they thought important. Having support/care plans turned down also compounded uncertainties about what were and were not legitimate uses for personal health budgets; one or two participants had accessed websites about the pilots and knew that such items had been allowed for other budget holders.

\section{Recommendation: Greater clarity is needed regarding what types of services and support people can secure with their personal health budget.}

\subsubsection{Getting approved services in place}

The internet, shops, friends and relatives were all consulted to find suitable services or items. Participants who were helped by health professionals to source items or services said they appreciated being able to avoid the 'legwork' of investigating providers.

Participants who used their budget to pay for services or items from a local list or 'menu' usually experienced few delays with procurement or supply. However, others experienced significant delays, especially when the pilot sites took responsibility for finding and procuring non-standard items or services. Participants' frustration was compounded by poor communication and difficulties in getting updates on progress; other participants were reluctant to 'pester' staff for information. Equally frustrating were situations where participants knew exactly what they wanted (for example, a mobile 
phone or home exercise equipment) and could have purchased it cheaply themselves but had to wait for the pilot site to source it elsewhere. Such delays led to some participants purchasing items with their own money or taking undue risks. Delays also impacted on other family members: for example, where budgets were being used to fund (additional) paid care or respite care. As personal health budgets move from pilot to mainstream, it will be important for procurement processes to be streamlined, for one-off as well as standard items.

\section{Recommendation: Procurement processes for personal health budgets should be streamlined, for one-off as well as standard items.}

\subsubsection{Personal health budgets and social care support}

Around one in eight budget holders and a majority of the carers in the sub-sample also had social care funding as well as their personal health budget. Only a minority were able to manage both their social care personal budget and their personal health budget through a single bank account. Others had to keep the two budgets entirely separate. Moreover, there were instances of considerable confusion, particularly among carers supporting people with progressive or very complex conditions, about what could be funded from each budget. For example, one carer reported disputes between the local authority and PCT over the funding of a new wheelchair for her severely disabled daughter:

"It's sold as if you've got much more control... but when it comes down to it, we've had all this trouble in getting it approved to buy an electric wheelchair which [daughter] has been assessed as needing" (carer of budget holder).

Other participants experienced problems when arrangements for managing personal health budgets received as direct payments were incompatible with well-established arrangements for managing their social care direct payment. Instances were reported of pilot sites requiring new references and criminal records bureau checks (CRB) for paid carers who had been employed for many years, or insisting on different accounting and payroll systems from those used for social care direct payments:

"I think it's going to be more time-consuming [than social care direct payments], definitely... They want receipts and they want proof every six months - I will have to send in bank statements, slips of the money being used" (budget holder).

For those more severely disabled budget holders and carers receiving both personal health budgets and social care personal budgets, arrangements appeared to work best when the former could be integrated with their established bank accounts, accounting and payroll arrangements.

\section{Recommendation: Accounting procedures for personal health budgets should be better co- ordinated with those of social care personal budgets, where relevant.}

\subsubsection{Budget holders' and carers' experiences of deployment options}

When interviewed three months after the offer of a personal health budget, only a minority of participants recalled being offered a choice of how their budget would be managed. Others recalled being told that their budget would be managed by the PCT or a third-party organisation; being told 
that a direct payment was not possible for them; or that it had been 'assumed' they would want a direct payment, particularly if the personal health budget was for NHS Continuing Healthcare.

Those who did recall being offered a choice of deployment options generally felt well supported, with adequate information about the different options. The most common information gaps concerned setting up special bank accounts and paying staff; one interviewee questioned how 'Mr or Mrs Average' was supposed to cope with these responsibilities.

The nine-month interviews explored budget holders' and carers' actual experiences of different deployment options. PCT or third-party management was valued because it removed any risk of the budget being used inappropriately; avoided additional responsibility and stress; and offered better opportunities for securing good deals and bargains. On the other hand, some participants whose budget was managed by the PCT without having had a choice of deployment option felt they were not trusted: 'They don't think you can look after it yourself ... so ... we'll look after it.' PCT or thirdparty management also involved greater risk of delays in the procurement of goods or paying for services: for example, one budget holder was deeply embarrassed when she was told at the gym that her fees had not been paid by the PCT. In retrospect, some participants said they would have preferred a direct payment so they could obtain items more quickly themselves.

In contrast, those who had direct payments generally felt this had been the right choice for them, despite relatively common initial difficulties in setting up bank accounts, and delays with the PCT paying money into designated accounts. Most participants using direct payments were employing their own carers or personal assistants. Some had not needed any help with recruitment as they had previous social care-funded carers in place, while others employed friends or relatives. For those recruiting carers for the first time, help from PCT staff or support workers with advertising and interviewing applicants, obtaining CRB clearance and drawing up employment contracts was greatly appreciated, 'taking a weight off my shoulders'. Even so, some recruitment problems were reported in finding suitable people who were willing to work on a payroll basis and not 'cash in hand'. Ongoing back-up support with employing carers was also highly valued, in case new employment arrangements broke down.

\section{Recommendation: Procurement and recruitment support for personal health budget holders (especially direct payment options) are valued by recipients.}

\subsection{Personal health budget initial set up and configuration}

As with any major system organisation reform, the introduction of personal health budgets in the pilot sites presented a range of challenges in getting the new systems in place and also securing sufficient 'cultural' acceptance of a substantially new way of working. To some extent the initial implementation of personal health budgets was hampered by a number of external factors, not least of which was the wholesale restructuring of the health system during the study period. Although these external factors would not necessarily apply to a future roll-out of personal health budgets, they were relevant to the process by which the pilots arrived at a functioning personal health budgets model. 
During the study period, a number of implementation issues were raised as contributing to the delays within the pilot process. In addition to the restructuring that organisational representatives faced, it was questioned whether the degree of cultural change had been under-estimated at the beginning of the pilot process.

Organisational representatives who were interviewed at an early stage of implementation suggested a number of factors that would facilitate future implementation, including:

- Acknowledging the importance of engaging with all representatives during the early implementation phase, including stakeholders, patients, clinicians, middle managers and chief executives;

- Developing adequate internal systems and processes to support personal health budgets;

- Acknowledging and managing the cultural change in terms of:

- The immediate impact on the workplace: for example, the capacity to implement a new initiative;

- Training needs for frontline staff;

- Identifying the concerns of the middle managers as it was feared that their views could be communicated to other representatives in the local area;

- Identifying and addressing concerns among frontline staff that could have the potential to delay the implementation process and also have an impact on the experiences of budget holders and their families.

Representatives also expressed a view that setting up personal health budget systems would benefit from Department of Health guidance, especially regarding:

- How to set budgets;

- Which services could be included in the personal health budget;

- How to facilitate choice, including the importance of market development to be able to offer real choice to budget holders.

We were unable to test these specific suggestions in the main quantitative analysis, although they appear generally consistent with the findings regarding the personal health budget process - see section 9.3.2.1.

Recommendation: Regarding initial set-up of personal health budget systems, organisational representatives indicated the benefits of: tackling cultural change issues among staff with good communication; engaging with all representatives; and developing adequate internal systems.

Recommendation: that the Department of Health provides further guidance as to budget setting, coverage and the facilitation of choice.

\subsection{The wider impact of personal health budgets}

The main analysis has concentrated on the impact of personal health budgets on the individual recipient and their family (carers). The third interim report (Jones et al., 2011) assessed the costs of implementing and operating the personal health budget pilot. There are also wider impacts to 
consider, including the consequences of moving beyond the pilot stage for local health and care economies. The main study was not designed to address these broader issues, but we can draw out some implications in terms of the changing balance of services that might result and which types of providers might be commissioned by budget holders.

\subsubsection{Impacts beyond the individual/family}

\subsubsection{Impact on commissioning staff}

Although the study was not designed to estimate comparable transaction costs for the personal health budget group and the control group, we did ask about the staffing of project teams in the PCT (as was) in interviews with project leads. New appointments, rather than changed staff roles or secondments, might indicate an increase in the costs of running personal health budget systems. It is nonetheless difficult to distinguish one-off set up costs from recurrent transaction costs in this respect. Furthermore, for a pilot there may be double running costs as both conventional and personal health budget systems are maintained. The third interim report (Jones et al., 2011) outlined that an overall average cost of $£ 93,280$ (median $£ 81,680$ ) within the first year would be required to implement the initiative. This cost would be in addition to what would have been incurred without personal health budgets within the health authority.

\subsubsection{Change in services accessed}

Budget holders did change the mix of services they secured with their budget. We found significant increases in the use of well-being services and other health services, such as specialised continuing healthcare. We also saw a reduction in the use of hospital care by the personal health budget groups compared to the control group. The study did not, however, show a change in the use of social caretype services between personal health budget and control groups. These results suggest, overall, that the use of personal health budgets was associated with a change in the balance between secondary and primary health on the one hand, and either community health or social care on the other.

The changes involved were relatively modest, but they do suggest that personal health budget holders had slightly different priorities than commissioners working on behalf of people in the control group. While the exact causes are difficult to tease out, the results imply that service use across different service categories was inter-related. There are tentative implications for policy around integration; the results could be interpreted as implying that greater integration would lead to a change in the balance of services used, and also that personal health budgets might be a vehicle to promote better integration.

Recommendation: Personal health budgets should be considered as a vehicle to promote greater service integration (especially where social care personal budgets and personal health budgets could be integrated around established bank accounts, accounting and payroll arrangements).

We did not find a significant effect of personal health budgets on the demand for informal care support. In this respect, costs to the family would not appear to be greatly affected in the longer run. This result is somewhat at odds with our initial expectations. It is worth cautioning that our main results regarding carers were in relation to the amounts of informal care used by personal health 
budget holders. The data we had about the impact on carers of cared-for people using personal health budgets was much more limited.

\subsubsection{Change in provider type}

The use of personal health budgets might also be expected to impact on which organisations are commissioned by the budget-holder to deliver services. In as far as the use of personal health budgets leads to a change in what sorts of services people might want to commission, we would also see a change in where these services and support are sourced. In particular, some types of services are not provided by NHS organisations and would have to be sourced externally. As regards social care in general, a very high proportion - around $90 \%$ - of mainstream publicly-funded services are commissioned from private and voluntary sector providers. ${ }^{53}$ We would expect that social carerelated services commissioned using personal health budgets aimed at meeting both health and social care needs will continue to be secured from the independent sector.

Primary and secondary health care services (classed as indirect expenditure in the main analysis - see chapter 6) are not funded from personal health budgets, so we would not see any change in the providers of these services. Other types of services - well-being services, community health services, therapy services and so on - could be sourced from non-public sector providers. We did not specifically gather information about the organisation providing the service. We did collect data on the proportion of personal health budget-funded expenditure that was made as a direct payment, and this might be indicative of the amount of expenditure that went to non-public providers in the study. ${ }^{54}$

As outlined in chapter 6 , this assumption about the spending of direct payment budgets underpinned an estimation of the change in the amount of service secured outside the NHS (and in-house social care providers) as a result of the introduction of personal health budgets. Two other key assumptions were made in this analysis. First, that irrespective of the use of personal health budgets, around $90 \%$ of social care was purchased from independent sector providers anyway. Second, that all well-being services were sourced outside of the NHS. With these assumptions, we found that the amount of expenditure going outside the NHS averaged $£ 2180$ per year greater for the personal health budgets group as compared to the control group. This figure corresponds to around $12 \%$ of the average level of direct expenditure in the personal health budget group ( $£ 18000$ per year). If we assume that only half of well-being services were sourced outside of the NHS, this figure reduces to $f 1900$ per year.

\footnotetext{
${ }^{53}$ See Allan, S. and J. Forder (2012). Care Markets in England - lessons from research. PSSRU, University of Kent, PSSRU Discussion paper: DP2815 (http://www.pssru.ac.uk/archive/pdf/dp2815.pdf).

${ }^{54}$ In theory the commissioning of publicly-delivered services would not require a monetary payment and so it is not unreasonable to expect that most direct payment budgets are used to purchase non-publicly-delivered services. Nonetheless, it might be possible that local arrangements are made to accommodate these sorts of transactions e.g. netting off the cost of public services from the direct payment. These arrangements ought to be classed as mixed notional and DP deployment in the data, but interpretation can differ between sites.
} 
These figures give us a sense of the wider impact of personal health budgets. They are made on the basis of assumptions that might reasonably be challenged, and that would affect the results. A systematic re-analysis of care plans and follow-up with participants would be needed to derive a more robust figure. There appears, nonetheless, some grounds to expect that the use of personal health budgets is likely to result in a change in the supply of services. On this basis we make the following recommendation.

Recommendation: Policy makers should anticipate that the use of personal health budgets is likely to result in a higher level of expenditure going to 'non-conventional' providers (for example, a greater use of non-NHS providers). Further research is required to better understand the scale of these changes. 


\section{References}

Alakeson, V. 2007. Putting Patients in Control: The case for extending self-direction in the NHS. London: Social Market Foundation.

Alakeson, V. 2010a. Active patient: The case for self-direction in healthcare. Sheffield: Centre for Welfare Reform; University of Birmingham. Health Services Management Centre.

Alakeson, V. 2010b. Issues in International Health Policy: International Developments in SelfDirected Care. U.S: Department of Health and Human Services.

Arksey, H. \& Kemp, P. A. 2008. Dimensions of Choice: A narrative review of cash-for-care schemes. York: Social Policy Research Unit, University of York.

Brown, G. 2008. Speech on the National health Service., 7 January [Online]. [Accessed].

Cabinet Office 2005. Improving the Life Chances of Disabled People. London: Cabinet Office Strategy Unit.

Carr, S. 2011. Personal budgets and international contexts: Lessons from home and abroad. Journal of Care Services Management, 5, 9-22.

Carr, S. \& Robbins, D. 2009. SCIE Research briefing 20: the implementation of indivdiual budget schemes in adult social care. London: Social Care Institute for Excellence.

Cole, D. 2009. Recovery Budgets in a Mental Health Service. Evaluating recovery budgets for people accessing an early intervention service and the imapct of working with selfdirected services on the team members within a North West of England NHS Trust. Liverpool: Merseycare NHS Trust.

Curtis, L. 2010. Unit Costs of Health \& Social Care, 2010. Personal Social Services Research Unit, University of Kent.

Curtis, L. 2011. Unit Costs of Health \& Social Care, 2011. Personal Social Services Research Unit, University of Kent.

Davidson, J., Baxter, K., Glendinning, C., Jones, K., Forder, J., Caiels, J., Welch, E., Windle, K., Dolan, P. \& King, D. 2012. Personal health budgets: Experiences and outcomes for budget hodlers at nine months. York: Social Policy Research Unit, University of York.

Department for Health 2008. NHS Next Stage review: Our vision for primary and community care. London: Department of Health.

Department of Health 2001. Valuing people: a new strategy for learning disability for the 21st century. . London: Department of Health.

Department of Health 2003. Direct Payment Guidance: Community Care, Services for Carers and Children's Services (Direct Payments) Guidance. London: Department of Health.

Department of Health 2009. Personal health budgets: first steps. London: Department of Health.

Department of Health 2010. Liberating the NHS: Greater Choice and control. A consultation on proposals. In: HEALTH, D. O. (ed.). London.

Department of Health 2012. Liberating the NHS: No decision about me, without me - Further consultation on proposals to secure shared decision-making. London: Department of Health.

Drummond, M. F. 2005. methods for the Economic Evaluation of Health Care Programmes, Oxfordshire, Oxford University Press.

Duffy, S. 2005. Individual budgets; transforming the allocation of resources for care. Journal of Integrated Care, 13, 8-16. 
Fox, D., Holder, J. \& Netten, A. 2010. Personal Social Servcies of Adult carers Carers in England 2009-10: Survey development project-Technical Report. PSSRU Discussion paper No. 2643(2). Canterbury: Personal Social Servcies Research Unit, University of Kent.

Glasby, J. \& Hasler, F. 2004. A healthy option? Direct payments and the implications for healthcare. . Birmingham: Health Services Management Centre/National Centre for Independent Living.

Glasby, J., Le Grand, J. \& Duffy, S. 2009. A healthy choice? Direct payments and healthcare in the English NHS. Policy and Politics, 37, 481-497.

Glasby, J. \& Littlechild, R. 2009. Direct payments and personal budgets: Putting personalisation into practice (2nd edition), Bristol, The Policy Press.

Glendinning, C., Arksey, H., Jones, K., Moran, N., Netten, A. \& Rabiee, P. 2009. The Individual Budgets Pilot Projects: Impact and outcomes for carers. York: Social Policy Research Unit, University of York.

Glendinning, C., Challis, D., Fernandez, J., Jacobs, S., Jones, K., Knapp, M., Manthorpe, J., Moran, N., Netten, A., Stevens, M. \& Wilberforce, M. 2008. Evaluation of the Individual Budgets Pilot Programme. Final Report. York: Social Policy Research Unit, University of York.

Glendinning, C., Haliwell, S., Jacobs, S., Rummery, K. \& Tyrer, J. 2000a. Bridging the gap: using direct payments to purchase integrated care. Health and Social Care in the Community, 8, 192-200.

Glendinning, C., Haliwell, S., Jacobs, S., Rummery, K. \& Tyrer, J. 2000b. Buying independence: Using direct payments to integrate health and social services. , Bristol, The Policy Press.

Grewal, I., Lewis, J., Flynn, T., Brown, J., Bond, J. \& Coast, J. 2006. Developing attributes for a generic quality of life measure for older people: Preferences or capabilities? Social Science and Medicine, 62, 1891-1901.

Higgs, P., Hyde, M., Wiggins, R. \& Blane, D. 2003. Researching quality of life in early old age: The importance of the sociological dimension. Social Policy \& Administration, 37, 239-252.

HM Government 2010a. Building the National Care Services. London: HM Government.

HM Government 2010b. Equity and Excellence: Liberating the NHS. London: HM Government.

HM Government 2011. Government response to the NHS Future Forum report. London: HM Government.

Holder, J., Smith, N. \& Netten, A. 2009. Outcomes and quality for social care services for carers: Kent County Council Carers Survey Development project 2007-2008. Internal Thechnical Report (not publicly available). Canterbury: Personal Social Services Research Unit, University of Kent.

Jones, K., Forder, J., Caiels, J., Welch, E., Windle, K., Davidson, J., Dolan, P., Glendinning, C., Irvine, A. \& King, D. 2011. The cost of implementing personal health budgets. Canterbury: Personal Social Services Research Unit, University of Kent.

Le Grand, J. 2007. The other invisible hand: Delivering public services through choice and competition, Princeton, N.J, Princeton University Press.

Leadbeater, C., Bartlett, J. \& Gallagher, N. (eds.) 2008. Making It Personal, London.

Macmillan Cancer Support. 2008. Macmillan Solutions [Online]. [Accessed]. 
Malley, J., Fox, D. \& Netten, A. 2010. Developing a carers' experience performance indicator. PSSRU Discussion Paper No. 2734. Canterbury: Personal Social Services Research Unit, University of Kent.

Maslow, A. H. 1968. Towards a psychology of being, Princeton, NJ, Van Nostrand. Milburn, A. 2007. RE: 'A 2020 vision for pubic services'. Speech at the London School of Economics and Political Science, 16 May.

Needham, C. 2011. Personalising public services: Understanding the personalisation narrative, London, Policy Press.

Needham, C. \& Carr, S. 2009. Co-production: an emerging evidence base of adult social care transformation. London: Social Care Institute for Excellence.

NHS Confederation and National Mental Health Development Unit 2009. Shaping personal health budgets: a view from the top. London: NHS Confederation and National Mental Health Development Unit.

NHS Confederation and National Mental Health Development Unit 2011. Personal health budgets: the veiws of service users and carers. London: NHS Confederation and National Mental Health Development Unit.

NICE 2007. Briefing paper for the methods working party on the cost effectiveness threshold. London: National Institute for Health and Clinical Excellence.

Nussbaum, M. 1995. Human capabilities, female human beings. In: NUSSBAUM, M. \& GLOVER, J. (eds.) Women, Culture and Development: a study of human capabilities. Oxford: Oxford University Press.

Nussbaum, M. \& Sen, A. (eds.) 1993. The quality of life, Oxford: Oxford University Press.

Pavolini, E. \& Ranci, C. 2006. Restructuring the welfare state: reforms in long-term care in Western European countries Journal of European Social Policy, 18, 246-259.

Raftery, J. 2009. NICE and the challenge of cancer drugs. British Medical Journal, 338.

Ritchie, J. \& Spencer, I. 1994. Qualitative data analysis for applied research. In: BRYMAN, A. \& BURGESS, R. G. (eds.) Analysing qualitative data. London: Routledge.

Robert Wood Johnson Foundation 2006. Choosing independence: An overview of the Cash and Counselling model of self-directed personal assistance servcies. . US: Robert Wood Johnson Foundation.

Rubin, D. B. 1987. Multiple imputation for nonresponses in surveys, New Jersey, Wiley.

Schalock, R. L. 2004. The concept of quality of life: what we know and do not know. Journal of Intellectual Disability Research, 48, 203-216.

Sen, A. 1982. Choice, Welfare and Measurement, Oxford, Blackwell.

Sen, A. 1993. Capability and well-being. In: NUSSBAUM, M. \& SEN, A. (eds.) The Quality of Life. Oxford: Clarenden Press.

The Health Foundation 2010. Personal health budgets: Research scan. London: The Health Foundation.

Timonen, V., Convery, J. \& Cahill, S. 2006. Care revolutions in the making? - a comparison of cash-for-care programmes in four European countries. Ageing \& Society, 26, 455-474.

Ungerson, C. \& Yeandle, S. (eds.) 2007. Cash for care in developed welfare states, Basingstoke: Palgrave Macmillan.

Weinstein, M. C. 1990. Principles of cost-effectiveness resource allocations in health care organisations. International Journal of Technology Assessment in Health Care, 6, 93103. 


\section{Evaluation of the personal health budget pilot programme}

\section{Appendices}

\section{Contents}

Appendices

Appendix A Methodological Framework ................................................................ 176

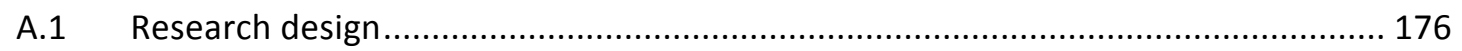

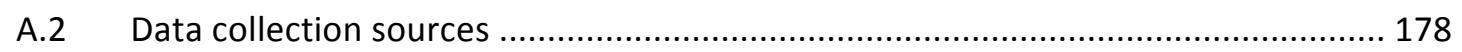

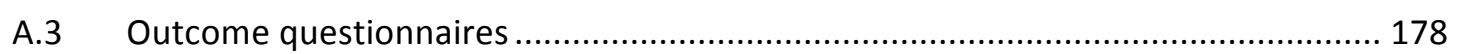

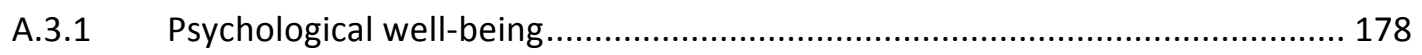

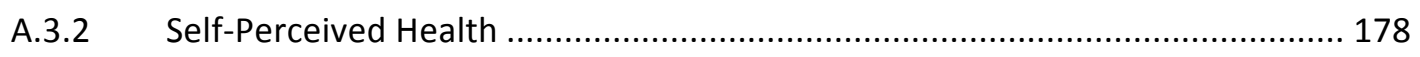

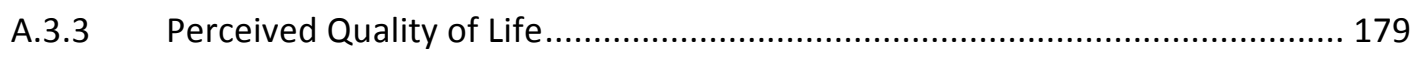

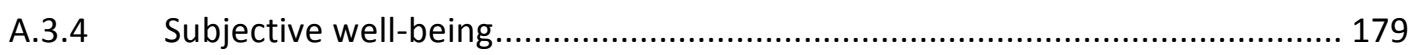

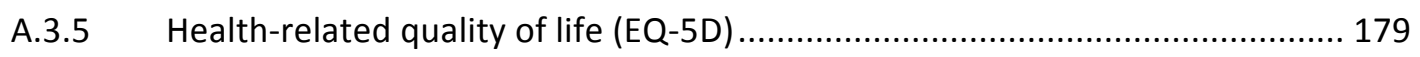

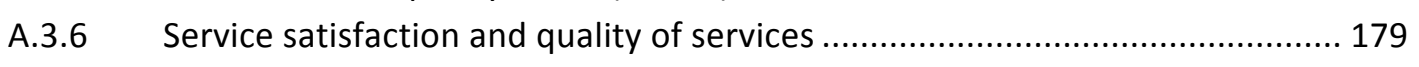

A.3.7 Care outcome-related quality of life (ASCOT) .............................................. 180

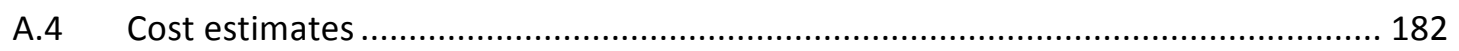

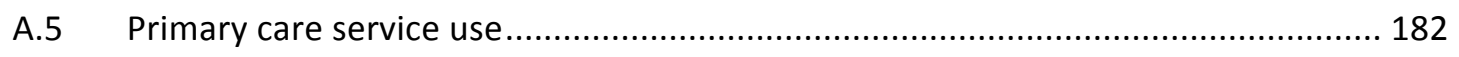

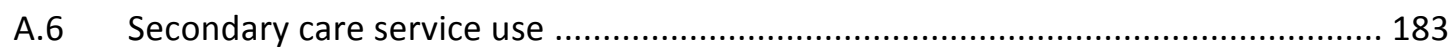

Appendix B.Research methods and sample: in-depth examination of personal health budgets from users' and carers' perspectives ............................................................ 185

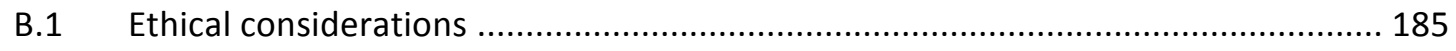

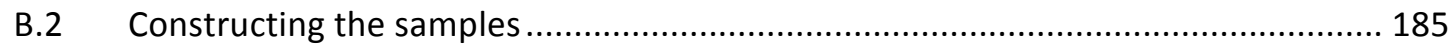

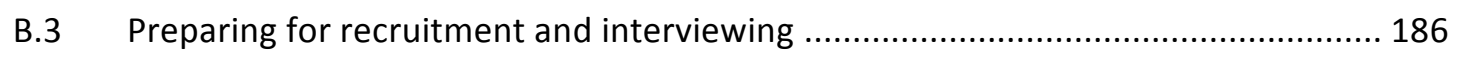

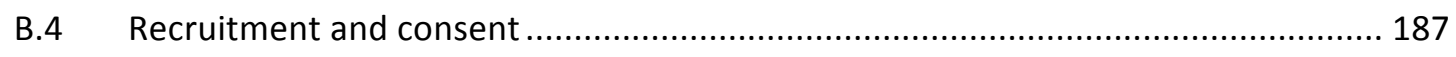

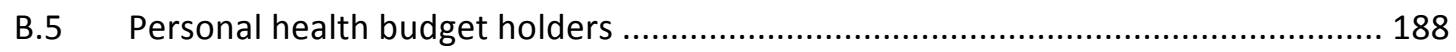

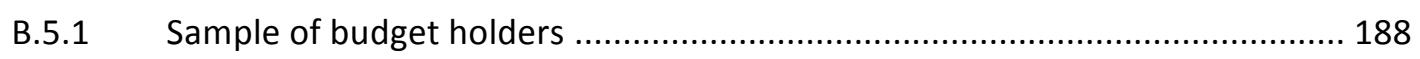

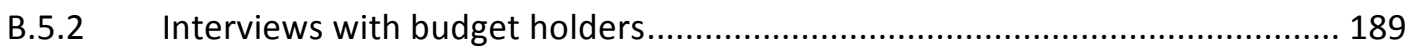

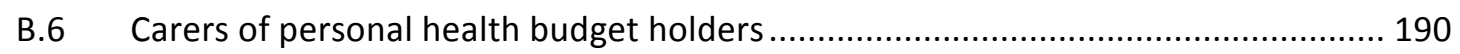

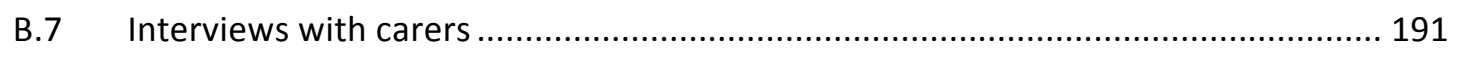

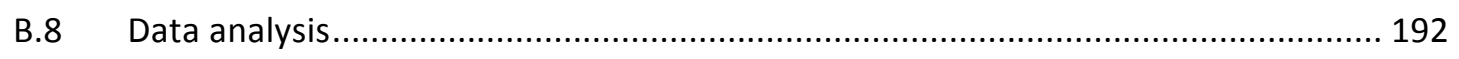

Appendix C Statistical Analysis ....................................................................... 193

C.1 Confounding variables included in the difference-in-difference multivariate analysis..

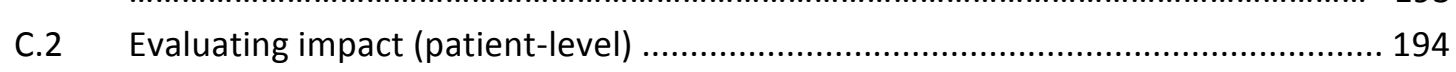




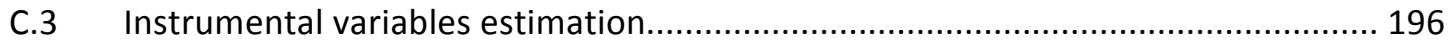

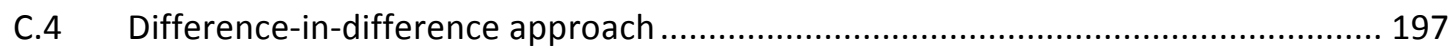

C.5 Mortality and confounding factors on outcome indicator changes ......................... 198

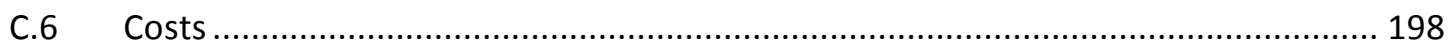

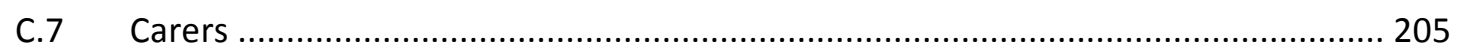

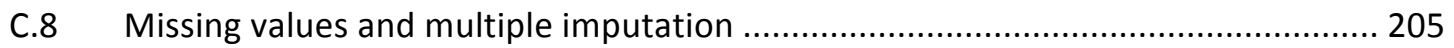

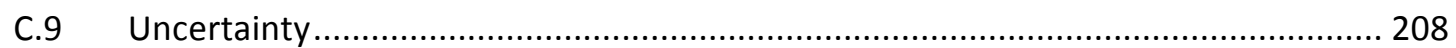

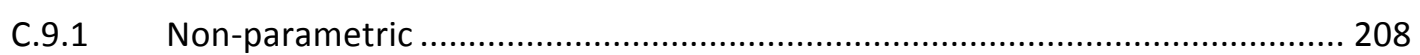

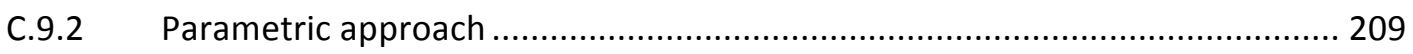

Appendix D Examples of quotes from organisational representatives following PHB

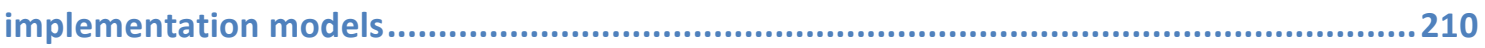

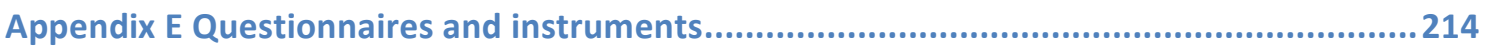

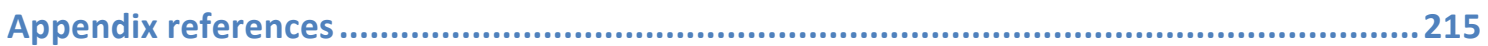

\section{Tables}

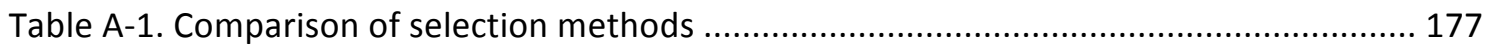

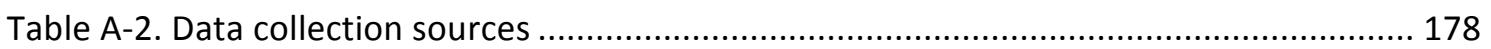

Table A-3. Options provided for each domain to reflect each need level............................... 181

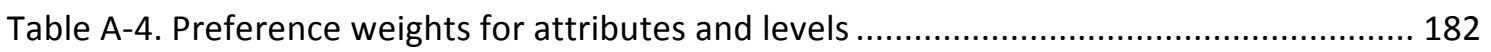



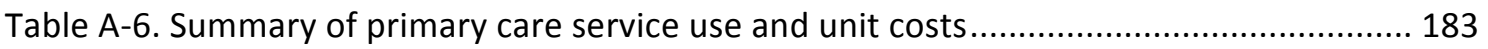

Table A-7. Summary of secondary care service use and unit costs ........................................ 184

Table B-1. Recruitment targets and sample: personal health budget holders......................... 189

Table B-2. Characteristics of sample: personal health budget holders .................................... 189

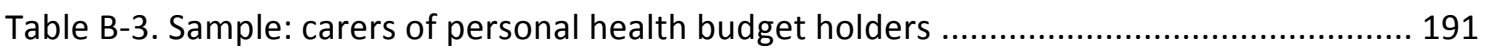

Table D-1. Quotes from organisational representatives implementing PHB model 1 ............. 210

Table D-2. Quotes from organisational representatives implementing PHB model 2 ............ 211

Table D-3. Quotes from organisational representatives implementing PHB model 3 ............. 212

Table D-4. Quotes from organisational representatives implementing PHB model 4 ............ 213 


\section{Appendix A Methodological Framework}

\section{A.1 Research design}

The evaluation within the in-depth sites used a controlled trial with a pragmatic design (depending on pilot site arrangements) to compare the experiences of people selected to receive personal health budgets with the experiences of people continuing under the current support arrangements for their condition. Two arrangements were used for sample allocation between intervention and control group:

- Randomisation. Where the pilot was set up so that personal health budgets were potentially offered by any health professional in the site, people who were judged as potentially eligible for a personal health budget were then randomised into either the personal health budget group or a control group.

- Stratification between different groups of health professions. In the main, personal health budget patients were drawn from practices in one patch within the PCT and the control patients from practices in a different patch (chosen to be as similar as possible). Where this was not possible, for example because local GPs refused to offer personal health budgets or where equivalent patches could not be easily found, control patients were drawn from practices selected on a more opportunistic basis.

The two arrangements have different implications for potential selection bias and 'contamination', as outlined in Table A-1. 
Table A-1. Comparison of selection methods

\begin{tabular}{|c|c|c|}
\hline Issues & $\begin{array}{l}\text { Individual level } \\
\text { randomisation method }\end{array}$ & Comparison method \\
\hline Selection bias & $\begin{array}{l}\text { Potential for selected sample as } \\
\text { eligible for PHB. Therefore possible } \\
\text { selected PHB and control } \\
\text { participants, which do not reflect } \\
\text { everyday practice and where the } \\
\text { generalising of conclusions will be } \\
\text { more limited. } \\
\text { No selection of patients into PHB or } \\
\text { control groups. Baseline } \\
\text { characteristics of sample should not } \\
\text { be different between PHB and } \\
\text { control groups. }\end{array}$ & $\begin{array}{l}\text { Potential for selected sample as } \\
\text { eligible for PHB. But control group } \\
\text { not chosen using PHB criteria; hence } \\
\text { PHBs compared to existing practice } \\
\text { and patients rather than a selected } \\
\text { group of control patients. } \\
\text { PHB and control groups likely to } \\
\text { have different characteristics for } \\
\text { operational reasons. } \\
\text { PHB and controls can be deliberately } \\
\text { selected into the PHB or control } \\
\text { group to influence evaluation } \\
\text { outcomes. } \\
\text { Difference-in-difference methods } \\
\text { can mitigate these problems. }\end{array}$ \\
\hline $\begin{array}{l}\text { Extra-patient } \\
\text { contamination }\end{array}$ & $\begin{array}{l}\text { PHBs are a systemic process centred } \\
\text { on the individual. Within any site } \\
\text { practitioners work with both PHB } \\
\text { and control group patients. } \\
\text { Implementation of PHB systems } \\
\text { might affect ('contaminate') the } \\
\text { operation of 'usual treatment' } \\
\text { alterative, undermining the validity } \\
\text { of the control group experience as a } \\
\text { counterfactual. }\end{array}$ & $\begin{array}{l}\text { Contamination less likely as PHB and } \\
\text { control group are drawn from } \\
\text { different groups of practitioners. }\end{array}$ \\
\hline $\begin{array}{l}\text { Loss-to-follow } \\
\text { up }\end{array}$ & $\begin{array}{l}\text { External factors that cause data to } \\
\text { be missing completely at random } \\
\text { (MCAR) less likely to be } \\
\text { differentiated between PHB and } \\
\text { control groups. } \\
\text { Missing at random (MAR) data more } \\
\text { problematic but can be imputed. }\end{array}$ & $\begin{array}{l}\text { MCAR missing data are less } \\
\text { problematic and can be imputed. } \\
\text { Missing at random (MAR) data more } \\
\text { problematic but can be imputed. }\end{array}$ \\
\hline
\end{tabular}




\section{A.2 Data collection sources}

Table A-2. Data collection sources

\begin{tabular}{|c|c|c|}
\hline & Data collection source & Timeline \\
\hline \multirow[t]{4}{*}{ Outcome data } & Participant self-report & Baseline \\
\hline & & 12 months after consent (main follow-up) \\
\hline & & 6 months after consent \\
\hline & & 24 months after consent \\
\hline \multirow[t]{2}{*}{ Health condition information } & Medical records & Baseline \\
\hline & & 12 months after consent \\
\hline \multirow[t]{2}{*}{ Primary care service use } & Medical records & Baseline \\
\hline & & 12 month after consent \\
\hline \multirow[t]{5}{*}{ Secondary care service use } & Hospital Episodes Statistics & $2009 / 2011$ \\
\hline & & $2010 / 2011$ \\
\hline & Participant self-report & Baseline outcome questionnaire \\
\hline & & Follow-up outcome questionnaire \\
\hline & Medical records & Baseline and follow-up \\
\hline \multirow{2}{*}{$\begin{array}{l}\text { Social care-related services- unit } \\
\text { costs }\end{array}$} & Participant self-report & Baseline outcome questionnaire \\
\hline & & Follow-up outcome questionnaire \\
\hline
\end{tabular}

\section{A.3 Outcome questionnaires}

The questionnaires contained a number of outcome indicators and measures.

\section{A.3.1 Psychological well-being}

The psychological well-being of service users was measured by the 12 -item version of the General Health Questionnaire (Goldberg, 1992) that explores whether respondents have experienced a particular symptom or behaviour over the past few weeks. Each item is rated on a four-point scale (less than usual, no more than usual, rather more than usual, or much more than usual). There are two scoring methods; the bi-modal ( 0 to 1 ) scoring style that indicates the likely presence of psychological distress according to a designated cut-off score of 4 or more; and the Likert scoring scale ( 0 to 3 ) which generates a total score ranging from 0 to 36 , with higher scores indicating worse conditions. The GHQ-12 has been extensively used in national studies including British Household Panel Survey and the Health Survey for England providing the scope for comparative analysis in the future.

\section{A.3.2 Self-Perceived Health}

A person's perception of his/her own health has been found to be a reliable predictor of functional decline (Ferraro, 1980), chronic disease (Shadbolt, 2007) and even mortality (Idler and Benyamini, 1997). The perceived health question was based on the five point scale suggested by Robine et al., (2003) as part of a European project on health indicators. This 
question asks respondents to rate their health in general according to five categories ranging from 'Very good' to 'Very bad'.

\section{A.3.3 Perceived Quality of Life}

The quality of life item was developed as part of a project funded under the ESRC Growing Older Research Programme (Bowling et al., 2002). This item was measured using a seven point scale, with categories ranging from 'So good, it could not be better' to 'So bad, it could not be worse' (Bowling, 1995).

\section{A.3.4 Subjective well-being}

We used a subjective global measure based on the measure used by ONS in the Integrated Household Survey (IHS). This measure used in this study seeks to capture general life happiness and satisfaction. We used a scale that considers satisfaction with life, happiness and satisfaction/worry about the person's health (Dolan et al., 2010). This measure consists of five questions using an eleven-point scale (ranging from 0-10). The questions are below:

- Overall, how satisfied are you with your life?

- Overall, how happy did you feel yesterday?

- Overall, how worried did you feel yesterday?

- Overall, how satisfied are you with your health?

- Overall, how worried are you about your health?

\section{A.3.5 Health-related quality of life (EQ-5D)}

The three-level Euro-QoL (EQ-5D) measure was used to explore the impact of the projects on users' reported changes in health related quality of life. There are three parts to this measure.

Part 1: Participants are asked to indicate what level of difficulty they have in carrying out five tasks; Mobility, Self-Care, Usual Activities, Pain/Discomfort and Anxiety/Depression. The levels of difficulty are, 'no problems', 'some problems' and 'extreme problems/unable'.

Part 2: Participants are asked to say how they feel their 'general level' of health has changed compared to the previous 12 months, whether it has got better, is much the same, or worse.

Part 3: Participants are asked to indicate how good or bad their heath state is on a 'thermometer' that runs from 0 (worst imaginable health state) to 100 (best imaginable health state).

\section{A.3.6 Service satisfaction and quality of services}

Measures of service satisfaction were based on quality indicators derived from the extensions to national user experience surveys for older home care service users and younger adults (Jones et al., 2007; Malley et al., 2006). 


\section{A.3.7 Care outcome-related quality of life (ASCOT)}

The Adult Social Care Outcomes Toolkit (ASCOT) was used to capture care-related quality of life measure that measure people's achievement of everyday activities, including basic capabilities such as dressing and feeding, as well as more complex capabilities such as feeling safe, being occupied and having a sense of control. ASCOT recognises that achievement of these activities may come from the support of services and interventions, as well as personal functioning. Whilst it was developed for people using long-term social care, many of the indicators are highly relevant for people with long-term conditions in general. For example, it would be expected that having a sense of control over daily living would be equally important for patients with a long-term health condition as well as for service users with social care needs.

The questions ask respondents to choose from a series of three deteriorating situations.

Table A-3 below shows the responses actually used in the interview. Rather than assuming that each domain and level is of equivalent importance the measure is weighted using population based preferences (see Burge et al., 2006). 
Table A-3. Options provided for each domain to reflect each need level

\begin{tabular}{|c|c|c|}
\hline Domain & $\begin{array}{l}\text { Need } \\
\text { level }\end{array}$ & Description \\
\hline \multirow[t]{3}{*}{ Control } & No & I have as much control over my daily life as I want \\
\hline & Low & Sometimes I don't feel I have as much control over my daily \\
\hline & High & I have no control over my daily life \\
\hline \multirow[t]{3}{*}{ Personal care } & No & I feel clean and wear what I want \\
\hline & Low & I sometimes feel less clean than I want or sometimes can't wear what I want \\
\hline & High & I feel much less clean than I want, with poor personal hygiene \\
\hline Food and & No & I eat the meals I like when I want \\
\hline \multirow[t]{2}{*}{ nutrition } & Low & I don't always eat the right meals I want, but I don't think there is a risk to my health \\
\hline & High & I don't always eat the right meals I want, and I think there is a risk to my health \\
\hline \multirow[t]{3}{*}{ Safety } & No & I feel as safe as I want \\
\hline & Low & Sometimes I do not feel as safe as I want \\
\hline & High & I never feel as safe as I want \\
\hline \multirow{3}{*}{$\begin{array}{l}\text { Social } \\
\text { participation }\end{array}$} & No & My social situation and relationships are as good as I want \\
\hline & Low & Sometimes I feel my social situation and relationships are not as good as I want \\
\hline & High & I feel socially isolated and often feel lonely \\
\hline \multirow{3}{*}{$\begin{array}{l}\text { Activities/ } \\
\text { occupation }\end{array}$} & No & I do the activities I want to do \\
\hline & Low & I do some of the activities I want to do \\
\hline & High & I don't do any of the activities I want to do \\
\hline \multirow[t]{3}{*}{ Accommodation } & No & My home is as clean and comfortable as I want \\
\hline & Low & My home is less clean and comfortable than I want \\
\hline & High & My home is not at all as clean or comfortable as I want \\
\hline \multirow{3}{*}{$\begin{array}{l}\text { Level of worry } \\
\text { and concern }\end{array}$} & No & I feel free from worry and concerns on a day-to-day basis \\
\hline & Low & I sometimes feel worried and concerned \\
\hline & High & I feel very worried and concerned on a daily basis \\
\hline \multirow{3}{*}{$\begin{array}{l}\text { Dignity and } \\
\text { respect }\end{array}$} & No & I am treated by other people with the dignity and respect that I want \\
\hline & Low & Sometimes I am not treated by other people with the dignity and respect that I want \\
\hline & High & I am never treated with the dignity and respect that I want \\
\hline
\end{tabular}

Table A-4 gives the weights that we applied to each of the attributes. For example, if a person reported that their personal cleanliness was at a desired level then this would be scored at $4.54^{1}$.

\footnotetext{
${ }^{1}$ Further information concerning the development and preference weights for the ASCOT quality of life measure can be found at the following website - http://www.hta.ac.uk/fullmono/mon1616.pdf
} 
Table A-4. Preference weights for attributes and levels

\begin{tabular}{lrrr}
\hline & Desired & Adequate & Poor \\
\hline Control over daily life & 5.18 & 1.5 & 0 \\
\hline Personal cleanliness & 4.54 & 1.87 & 1.09 \\
\hline Meals and nutrition & 4.16 & 2.59 & 1.96 \\
\hline Safety & 4.71 & 1.71 & 1.14 \\
\hline Social participation & 4.67 & 2.36 & 0.76 \\
\hline Activities/occupation & 4.50 & 3.95 & 1.69 \\
\hline Home cleanliness and comfort & 4.38 & 2.47 & 1.76 \\
\hline Anxiety & 4.69 & 1.88 & 1.24 \\
\hline Dignity and respect & 4.25 & 1.63 & 1.18 \\
\hline
\end{tabular}

\section{A.4 Cost estimates}

Table A-5 shows the social care unit data that was taken from the PSS EX1.

Table A-5. Summary of the social care cost estimates

\begin{tabular}{|c|c|c|}
\hline & $\begin{array}{l}\text { Average unit cost } \\
2009 / 2010\end{array}$ & $\begin{array}{l}\text { Average unit } \\
\text { cost } 2010 / 2011\end{array}$ \\
\hline Day care - older person & $£ 30$ & $£ 34$ \\
\hline $\begin{array}{l}\text { Day care- younger person (average cost between mental } \\
\text { health, learning disability and physical disability) }\end{array}$ & $£ 67$ & f73 \\
\hline Home care per hour (local authority) & $£ 38$ & $£ 42$ \\
\hline Home care per hour (delivered by others) & $£ 15$ & $£ 15$ \\
\hline Meals on wheels & f8 & $£ 4$ \\
\hline Residential care ${ }^{2}$ & $f 580$ & £633 \\
\hline Local authority social worker ${ }^{3}$ & $£ 55$ & $£ 53$ \\
\hline
\end{tabular}

\section{A.5 Primary care service use}

The outcome questionnaire and the medical record template collected information about participant's primary care service use at two time points: baseline and at 12 months after

\footnotetext{
${ }^{2}$ Average gross weekly expenditure per person on supporting adults and older people in residential and nursing care (including full cost paying and preserved rights residents) and providing intensive home care

${ }^{3}$ Based on an hour of face to face contact.
} 
consent date. Table A-6 shows the national unit costs that were used for these services (Curtis, 2011; Curtis, 2010).

Table A-6. Summary of primary care service use and unit costs

\begin{tabular}{lrr}
\hline Service resource & $\begin{array}{c}\text { Unit cost } \\
\text { 2009/2010 }\end{array}$ & $\begin{array}{c}\text { Unit cost } \\
\text { 2010/2011 }\end{array}$ \\
\hline $\begin{array}{l}\text { District nurse, health visitor or other kind of nurse } \\
\quad \text { Home and clinic }\end{array}$ & $£ 38^{5}$ & $£ 41$ \\
$\begin{array}{l}\text { Occupation therapist, physiotherapist, speech } \\
\text { therapist or any other kind of therapist }\end{array} \quad$ & & \\
$\quad \begin{array}{l}\text { Home and clinic } \\
\text { General Practitioner } \\
\quad \text { Surgery } \\
\text { Home }\end{array}$ & & $£ 31$ \\
\hline
\end{tabular}

\section{A.6 Secondary care service use}

Information concerning secondary care service use was extracted at two time-points from the Hospital Episodes Statistics database (NHS Information Centre). We collected admitted patient, outpatient and A\&E activity data for each study participant using their NHS number. Due to the time lag in the availability of HES data, some activity was truncated for those participants joining the study at the end of the recruitment period. Data from medical records was used for any missing period rather than HES - see section C.6.1.1 below for details of this calculation.

Inpatient data were costed by applying the $2008 / 9$ admitted patient tariff. We calculated the cost of individual spells for participants using either the elective or non-elective tariff for the corresponding HRG of the spell. Using data on bed-days for the spend, we adjusted for elective and non-elective long-stays applying the long-stay tariff after the relevant trim-point for that HRG and admission type. Short-stay reductions were also applied. The total annual cost of inpatient care per study participant was then calculated by summing up the cost of individual spells. Total costs for different years were inflated using the weighted mean costs for the corresponding year.

\footnotetext{
${ }^{4}$ Based on an average unit cost per hour in clinic of between a community nurse (including a district nursing sister and district nurse) and health visitor.

${ }^{5}$ Based on an hour of client contact.

${ }^{6}$ Based on an average unit cost per hour in clinic between a hospital physiotherapist, community physiotherapist, community occupational therapist and a community speech and language therapist

${ }^{7}$ Based a clinic consultation lasting 11.7 minutes including direct care staff costs.

${ }^{8}$ Based on a home visit lasting 11.7 minutes including 12 minutes for travel and direct care staff costs.
} 
Outpatient attendances were collected for study participants, by type of attendance. An average cost per attendance was applied at $£ 136$ for 2009/10 and $£ 147$ for 2010/11 (taken from the Unit Costs report, Curtis 2011 and 2012). Total annual costs per person were calculated by summing attendances.

Accident and Emergency attendances were also collected for study participants, by type of A\&E attendance as categorised using the 10 A\&E tariff codes (2010/11 tariff). The corresponding A\&E tariff rate was applied and the totals deflated for the relevant year. We also added in ambulance transport using HES data on arrival mode per patient. Total yearly cost per study participant was calculated by summing attendances and transport costs.

Table A-7 provides a summary of the unit costs used in these calculations.

Table A-7. Summary of secondary care service use and unit costs






\section{Appendix B Research methods and sample: in-depth examination of personal health budgets from users' and carers' perspectives}

This appendix gives details of the recruitment and interviewing of personal health budget holders and carers.

The in-depth strand as a whole had two main elements:

- Interviews with budget holders at approximately three months and nine months after taking up the personal health budget;

- Interviews with carers of budget holders at approximately three months and nine months after taking up the personal health budget.

\section{B.1 Ethical considerations}

The wider evaluation received ethical approval from an NHS Research Ethics Committee; no additional approvals were required for the in-depth strand. However, in accordance with good practice, separate information sheets and consent forms specific to the in-depth research strand were prepared for participants. A number of preparatory measures (detailed below) were also carried out, in order to ensure appropriate and sensitive conduct of the in-depth interview research.

\section{B.2 Constructing the samples}

The sampling frame was provided by the wider personal health budget evaluation. The research team at the University of Kent supplied the SPRU research team with a database of all individuals recruited to the personal health budget evaluation. Transfer of the database was done electronically via a secure virtual private network. This database was updated periodically, allowing the SPRU team to construct a sample iteratively, according to the recruitment targets for each sub sample and health condition and also balanced for other characteristics including age and gender. The primary sampling criterion for the budget holders was the individual's health condition; secondary criteria were a spread of ages and an approximately even gender balance. The intention was also to include a range of ethnic groups within the in-depth sub-samples. However, this was dependent on the wider sample and, given the small numbers of non-White British budget holders in the evaluation at the time that people were being approached for the interviews, the potential number of minority ethnic interviewees in the in-depth sample was similarly limited.

The researchers aimed to include participants from at least half of the 20 in-depth evaluation sites in order to obtain a variety of local experiences. The range of health conditions being covered in the different pilot sites also guided sampling decisions. Most sites were offering personal health budgets to only two or three condition groups and their overall recruitment targets also varied, meaning that some sites offered more potential for in-depth sample 
selection than others. Only one of the sites offered personal health budgets for maternity services.

Regarding deployment options for personal health budgets, the intention was to include some budget holders who were receiving direct payments. However, this was again dictated by the number of pilot sites offering direct payments at the time of fieldwork and the numbers of budget holders taking up this deployment option.

\section{B.3 Preparing for recruitment and interviewing}

Prior to beginning fieldwork, the two researchers conducting the in-depth interviews held an informal meeting with a small group of colleagues in SPRU who had substantial collective experience of interviewing people with long-term and severe health conditions. This meeting highlighted a number of practical and ethical considerations to take into account when arranging and conducting the interviews. These included, for example, the most appropriate times of day to interview people with fluctuating conditions or complex medication regimes; the importance of pacing and breaks for participants who could tire easily; how to respond if a participant became unwell or distressed; and appropriate ways to involve other parties who might wish to be present during interviews.

A key source of background information in preparing for the semi-structured interviews was the personal health budget lead in each of the 20 in-depth pilot sites. When details of budget holders and carers were provided to the SPRU team by the University of Kent, the SPRU researchers contacted the personal health budget lead in each site to gather up-to-date information on the personal, health and wider circumstances of each budget holder or carer who was being considered for recruitment to the in-depth interview sub-sample. The purpose of these preliminary enquiries was to avoid making approaches to budget holders or carers if their current circumstances suggested this would be inconvenient or inappropriate and to ensure that the researchers were aware of any potential risks to their own wellbeing posed by that budget holder's circumstances.

The researchers first sent an introductory email to personal health budget leads explaining the reason for their contact, and then made a telephone call to gather key information including:

- The main health condition for which the person was receiving a personal health budget.

- Any recent changes in health or personal circumstances that should be taken into account before making contact or making a home visit.

- Any language or communication needs that should be taken into account when making initial contact and carrying out a research interview.

Personal health budget leads and care navigators were invariably very helpful in responding to these requests for information. Sometimes personal health budget leads identified some budget holders as particularly 'good' people to approach. However, the researchers attempted to set aside this type of information so as not to construct a sample biased towards the most 
articulate, positive or amenable respondents. Personal health budget leads and care navigators also commented on the likelihood of the budget holder being capable of engaging in an in-depth interview of the type intended for this strand of the evaluation. On the basis of the above range of information, the researchers made decisions about who to approach with an invitation to take part in an in-depth interview.

\section{B.4 Recruitment and consent}

Having identified potential participants, recruitment proceeded with an initial invitation letter and information sheet sent to selected budget holders or carers. All budget holders and carers had been informed when recruited to the wider evaluation that they may be contacted about taking part in some more in-depth research. However, being aware that not all participants would recall this clearly, the invitation letter reiterated why participants were being contacted and the information sheet set out comprehensive details of what participation in the in-depth strand of the study would involve.

Invitation letters were sent out in several successive waves, in part to pace the fieldwork but also because of the initially slow recruitment of participants to the wider evaluation. These waves were timed to correspond to three months after being offered a personal health budget for budget holders and carers, and three months after the birth of their baby for maternity personal health budget holders. Experience of the Individual Budgets Pilot evaluation also suggested that it would be worth undertaking some interviews for later on in the pilots, when personal health budgets pilot sites' recruitment and care/support planning processes had 'bedded in'.

In the first wave of invitation letters for budget holders, the researchers offered a two-week opt-out period, during which budget holders could state (by pre-paid postal reply slip, telephone or email) if they did not wish for further contact from the researchers. The first six invitation letters sent out included this option, but no opt-outs were received during the twoweek period. Following the two-week opt-out period, the researchers contacted budget holders by telephone to ask if they had any further questions and to see if they would be willing to participate in a research interview. Of these initial six approaches, four participants agreed to interview, one opted out at the point of telephone contact and one was not able to be contacted by telephone. Of those who agreed to be interviewed, most did not have a clear recall of the invitation letter by the time of the researcher's telephone call, though they still expressed willingness to take part in an in-depth interview. Based on this experience, the research team decided that a two-week opt-out period was unnecessary for future invitations and invitation letters were amended to say that a researcher would be in touch by telephone to talk about possible participation.

Budget holders and carers who chose not to take part in an in-depth interview did so because of severe ill health, current personal circumstances (for example, moving house), too many other time commitments, or simply feeling that it would be too demanding. In a number of cases where the budget holder was very unwell, frail or had communication difficulties, the 
initial telephone conversation took place with a third party (typically a partner) and, in such cases, it was usually established that it would be inappropriate to conduct an in-depth interview with the budget holder. Wherever possible, however, the researchers endeavoured to speak to the budget holder themselves before deciding not to pursue an interview.

The following three sections give specific details on the samples and interviews with personal health budget holders, budget holders for maternity services and carers, respectively.

\section{B.5 Personal health budget holders}

\section{B.5.1 Sample of budget holders}

The study aimed to interview a sample of 55 personal health budget holders. The timing of the three month interview was intended to allow for reflection on the decision-making and care/support planning process at a point in time when participant recall remained relatively good but sufficient time had elapsed for some care/support planning to have taken place. ${ }^{9}$

Six health conditions would be covered by the budget holder sample: diabetes, chronic obstructive pulmonary disease (COPD), stroke, long-term neurological conditions (for example, multiple sclerosis, Parkinson's), mental health conditions and people receiving NHS Continuing Healthcare. Target recruitment numbers were agreed in advance. Table B-1 gives the target numbers and the number of budget holders actually recruited and interviewed for each condition.

\footnotetext{
${ }^{9}$ Previous experience of the individual budgets pilot evaluation suggested that scheduling interviews any earlier than three months may mean that little planning had taken place with the budget holder.
} 
Table B-1. Recruitment targets and sample: personal health budget holders

\begin{tabular}{lcrc}
\hline Health condition & Recruitment target & $\begin{array}{c}\text { Interviews at } \\
\text { 3 months }\end{array}$ & $\begin{array}{c}\text { Interviews at } \\
\mathbf{9} \text { months }\end{array}$ \\
\hline COPD & 5 & 7 & 6 \\
\hline Diabetes & 5 & 6 & 5 \\
\hline Stroke & 10 & 8 & 8 \\
\hline Mental health & 10 & 9 & 8 \\
NHS continuing healthcare & 10 & 15 & 15 \\
\hline LTNC & 15 & 13 & 10 \\
\hline TOTAL & 55 & 58 & 52 \\
\hline
\end{tabular}

Table B-2 shows the characteristics of the 58 budget holders recruited and interviewed at three months.

Table B-2. Characteristics of sample: personal health budget holders

\begin{tabular}{|cc}
\hline Characteristic & 3 month interviews \\
\hline Age* range & 1 \\
\hline Teens & 8 \\
\hline $20 \mathrm{~s}$ & 11 \\
\hline $30 \mathrm{~s}$ & 7 \\
$40 \mathrm{~s}$ & 10 \\
\hline $50 \mathrm{~s}$ & 13 \\
\hline $60 \mathrm{~s}$ & 4 \\
\hline $70 \mathrm{~s}$ & 3 \\
\hline $80 \mathrm{~s}$ & \\
\hline Gender & 31 \\
\hline Female & 27 \\
\hline Male & \\
\hline
\end{tabular}

*1 person's age unknown.

\section{B.5.2 Interviews with budget holders}

The majority of interviews were conducted face-to-face in the budget holder's home, using a semi-structured topic guide. A small number of interviews were conducted by telephone. Three month interviews typically lasted 80-90 minutes; nine month interviews lasted about 6090 minutes. Several at both three and nine months approached two hours. Sometimes people 
chose to take a brief break but in most cases interviews continued uninterrupted for their duration.

The main topics covered in the three month interviews were:

- Health condition and satisfaction with services prior to taking up a personal health budget.

- $\quad$ Finding out about personal health budgets.

- Making the decision to try a personal health budget.

- The amount of the personal health budget and feelings about this.

- Deciding how to use the personal health budget.

- How the personal health budget is managed.

- Experiences so far of having a personal health budget.

The main topics covered in the nine month interviews were:

- The impacts of the personal health budget on the health, well-being and quality of life of the budget holder (and other family members).

- How the personal health budget had been used and budget holders' reflections on the choices they had made.

- Budget holders' satisfaction with the level of their personal health budget.

- Reflections on the chosen management options for the personal health budget.

- Experiences of the implementation of personal health budgets.

The researchers used the topic guides flexibly. While key themes were covered with all participants, certain questions or probes were omitted where not applicable to that person's circumstances or experience.

Although the researchers tried to avoid conducting interviews with 'proxy' respondents on behalf of a personal health budget holder, in a number of cases a third party was present during the interview and often contributed a substantial amount to the conversation.

Three and nine month interviews were digitally recorded with the participant's consent. Some participants showed printed papers to the researcher during the interview, for example, assessment forms, care/support plans, financial information or correspondence from the personal health budget lead or care navigator. These documents were discussed during the interviews as relevant but detailed information from such paperwork was not recorded and copies were not taken away by the researcher.

\section{B.6 Carers of personal health budget holders}

The evaluation aimed to conduct semi-structured interviews with 20 carers of personal budget holders, at three and nine months after the offer of a personal health budget. Although a number of carers had consented to participate in the main evaluation as proxy respondents for budget holders, they were not considered appropriate to interview, as they had not consented 
to participate as carers in their own right. Recruitment of carers (as respondents in their own right) to the main evaluation was delayed. Therefore, in order to be able to conduct both the three- and nine- month interviews within the overall timeframe of the evaluation, it was necessary to select the sample from a limited number of the earliest carer recruits.

Consequently, it was not possible to recruit carers supporting personal budget holders with the full range of conditions covered by the pilot sites, nor carers from all the sites involved in the evaluation. Nineteen carers were recruited for the in-depth interviews, a majority of whom were supporting personal budget holders with long-term neurological conditions (LTNC) or eligible for NHS Continuing Healthcare.

Subsequently there was some attrition from the sample: five carers withdrew from the personal health budget pilot and/or the evaluation before the nine-month interview and one who was interviewed at three months could not be contacted again at nine months. Table B-3 summarises the sample.

Table B-3. Sample: carers of personal health budget holders

\begin{tabular}{lcc}
\hline Carers who were supporting relatives with: & 3 month interview & 9 month interview \\
\hline Stroke & & 1 \\
\hline COPD $^{2}$ & 3 & 1 \\
LTNC $^{3}$ & 2 & 4 \\
\hline NHS Continuing Healthcare & 7 & 7 \\
\hline Total & 7 & 13 \\
\hline 1. & One of these had a long-term neurological condition as well as stroke. \\
2. & One of these had multiple health problems, including arthritis, as well as COPD. \\
3. $\quad$ Two of these had additional health problems, including diabetes and Aspergers Syndrome.
\end{tabular}

Fourteen carers were female and five were male. Their ages varied. At the time of recruitment eight people were in their forties; three in their fifties; five in their sixties and the remaining three were in their seventies.

To focus specifically on the impacts and outcomes of personal health budgets, the analysis concentrated only on the 13 carers who took part in both interviews. Even so, at nine months one of these carers had recently withdrawn from the personal health budget pilot; one was caring for someone still waiting to receive a personal health budget; and one was not sure whether a personal health budget had been awarded or not.

\section{B.7 Interviews with carers}

As with the main sample of budget holders, carers were interviewed using a semi-structured topic guide. The three-month interviews focused on:

- carers' early knowledge of personal health budgets; 
- their role in the decision to try a personal health budget and

- their role in planning, so far, how it would be used and managed.

The nine-month interviews explored:

- carers' experiences with the overall implementation of the personal health budget;

- their satisfaction with the roles they had been able to play in planning how the budget was to be used; and

- the direct and indirect impacts of the budget on their carer role.

The researchers used the topic guides flexibly. While key themes were covered with all participants, certain questions or probes were omitted where not applicable to that person's circumstances or experience. Both rounds of interviews were conducted face-to-face or by telephone and were digitally recorded.

\section{B.8 Data analysis}

The completed interviews for personal health budget holders, maternity services budget holders and carers of budget holders were transcribed in full. Data (summaries and quotations) was extracted from each transcript and placed in a template organised by themes. The themes included in the template followed closely the structure of the topic guide. The thematic analysis was supported using MaxQDA and used the Framework (Ritchie and Spencer, 1994) approach. The themes were written up for the sample as a whole, with each researcher taking lead responsibility for writing up a number of these. 


\section{Appendix C Statistical Analysis}

\section{C.1 Confounding variables included in the difference-in-difference multivariate analysis}

Table C-1. Confounding variables include in the difference-in-difference multivariate models

\begin{tabular}{ll}
\hline Demographic information & Socio-economic information \\
\hline Age & Highest education level \\
\hline Ethnicity & Benefit receipt \\
\hline Accommodation & Current circumstances \\
\hline $\begin{array}{l}\text { Gender (excluding trans-gender) } \\
\text { Household composition }\end{array}$ & Activities of daily living \\
\hline Employment status & Receipt of informal care support \\
\hline Marital status & Personal health budget process \\
\hline Health conditions - dummy codes & Personal health budget /Control Group \\
\hline NHS Continuing Healthcare & Implementation models 1 to 5 \\
\hline Diabetes & Time in receipt of services paid for by the \\
& personal health budget \\
\hline Mental health & Personal health budget value \\
\hline COPD & Deployment of the personal health budget \\
\hline Stroke & \\
\hline Long-term neurological & Interaction effects with each main effect \\
\hline & Group (personal health budget or Control \\
& Group) \\
\hline Other factors & Gender \\
\hline Time period between consent and 12- & Individual health conditions \\
month outcome interview & Marital status \\
\hline Area cost adjustment & \\
\hline Area classification - rural/urban & \\
\hline Deprivation scores & \\
\hline Help with the outcome interview & \\
\hline Pilot site dummy codes & \\
\hline
\end{tabular}

\footnotetext{
${ }^{10}$ The area cost adjustment reflects the varying costs of service delivery around the country.
} 


\section{C.2 Evaluating impact (patient-level)}

We started with a basic theoretical model, whereby a person's care-related quality of life is assumed to be determined by their needs-related characteristics (e.g. severity of their condition, levels of disability or impairment, age, sex and so on) and also the support and services they receive. Personal health budgets change the process by which care services are commissioned and used, and this should also have an impact on quality of life. A simple linear model can be expressed as:

$$
y_{j t}=\theta_{0}+\theta_{1} B_{j t}+\theta_{2} x_{j t}+\theta_{3} m_{j t}+\theta_{4} w_{j}
$$

where $y_{j t}$ is an outcome indicator such as an ASCOT or EQ-5D quality of life score. Also $B_{j t}$ is the care process i.e. whether a person has a personal health budget or not, $x_{j t}$ is expenditure on health and social care services, $m_{j t}$ is a vector of needs-related characteristics that affect people's underlying health condition and functioning, and $w_{j}$ are time-invariant needs-related characteristics (NRCs). The subscript $j$ denotes each patient and $t$ is time.

Services are deployed according to need, care process and other factors (denoted $x_{j t}^{0}$ )

$$
x_{j t}=\alpha_{0}+\alpha_{1} B_{j t}+\alpha_{2} m_{j t}+\alpha_{3} x_{j t}^{0}
$$

Similarly, NRCs are functions of care services and care processes, and other factors (denoted $\left.m_{j t}^{0}\right)$ :

$$
m_{j t}=\delta_{0}+\delta_{1} B_{j t}+\delta_{2} x_{j t}+\delta_{3} m_{j t}^{0}
$$

We solved these two equations for their partial reduced-forms:

$$
x_{j t}=\mu_{0}+\mu_{1} B_{j t}+\mu_{2} x_{j t}^{0}+\mu_{3} m_{j t}^{0}
$$

Similarly, NRCs are functions of care services and care processes, and other factors (denoted $\left.m_{j t}^{0}\right)$ :

$$
m_{j t}=\lambda_{0}+\lambda_{1} B_{j t}+\lambda_{2} x_{j t}^{0}+\lambda_{3} m_{j t}^{0}
$$

In turn, these functions could be substituted into (C-1):

$$
\begin{gathered}
y_{j t=\theta_{0}+\theta_{2} \mu_{0}}+\theta_{3} \delta_{0}+\left(\theta_{1}+\theta_{2} \mu_{1}+\theta_{3} \delta_{1}\right) B_{j t}+\left(\theta_{2} \mu_{2}+\theta_{3} \delta_{2}\right) x_{j t}^{0} \\
+\left(\theta_{2} \mu_{3}+\theta_{3} \delta_{3}\right) m_{j t}^{0}+\theta_{4} w_{j}
\end{gathered}
$$

To remove the time invariant factors, we took differences:

$$
\begin{aligned}
y_{j t+1}-y_{j t}= & \left(\theta_{1}+\theta_{2} \mu_{1}+\theta_{3} \delta_{1}\right)\left(B_{j t+1}-B_{j t}\right)+\left(\theta_{2} \mu_{2}+\theta_{3} \delta_{2}\right)\left(x_{j t+1}^{0}-x_{j t}^{0}\right) \\
& +\left(\theta_{2} \mu_{3}+\theta_{3} \delta_{3}\right)\left(m_{j t+1}^{0}-m_{j t}^{0}\right)
\end{aligned}
$$

We estimated this difference model in reduced-form as: 


$$
y_{j t+1}-y_{j t}=b_{0}+b_{1}\left(B_{j t+1}-B_{j t}\right)+\varepsilon
$$

where $b_{1}=\theta_{1}+\theta_{2} \mu_{1}+\theta_{3} \delta_{1}$. The constant, $b_{0}$, will capture any person-invariant part of the last two terms of (C-7), with the remaining components going into the error i.e.

$\varepsilon=\varepsilon\left(\left(x_{j t+1}^{0}-x_{j t}^{0}\right),\left(m_{j t+1}^{0}-m_{j t}^{0}\right)\right)$. Potentially an OLS estimation of (C-8) would produce a biased estimate of $b_{1}$ due to these omitted variables in that the selection of participation into the personal health budget or control groups was not completely blind and at random.

However, estimating this model in differences limits this problem in practice because the choice of $B_{j t+1}$ is made at time $t$ (baseline) not $t+1$. Whilst it might be possible that personal health budget group choice is influenced by $x_{j t}^{0}$ or $m_{j t}^{0}$, omitted variable bias would only arise if the differences $\Delta x_{j}^{0}=\left(x_{j t+1}^{0}-x_{j t}^{0}\right)$ and $\Delta m_{j}^{0}=\left(m_{j t+1}^{0}-m_{j t}^{0}\right)$ were correlated with $x_{j t}^{0}$ or $m_{j t}^{0}$ and in turn with the choice of $B_{j t+1}$. Furthermore, we can quite reasonably assume that the differences $\Delta x_{j}^{0}=\left(x_{j t+1}^{0}-x_{j t}^{0}\right)$ and $\Delta m_{j}^{0}=\left(m_{j t+1}^{0}-m_{j t}^{0}\right)$ are small (approaching timeinvariance) over the period of the study. In this case, (C-7) reduces to $y_{j t+1}-y_{j t}=\left(\theta_{1}+\right.$ $\left.\theta_{2} \mu_{1}+\theta_{3} \delta_{1}\right)\left(B_{j t+1}-B_{j t}\right)$ and an OLS estimation will be unbiased.

As a final safeguard, we also estimated a 'controlled' model:

$$
y_{j t+1}-y_{j t}=b_{0}+b_{1}\left(B_{j t+1}-B_{j t}\right)+b_{2} \tilde{x}_{j t}^{0}+b_{3} \widetilde{m}_{j t}^{0}+\varepsilon
$$

using baseline characteristics as proxies, $\tilde{x}_{j t}^{0}$ and $\tilde{m}_{j t}^{0}$, on the assumption that $\Delta x_{j}^{0}=\Delta x_{j}^{0}\left(\tilde{x}_{j t}^{0}\right)$ and $\Delta m_{j}^{0}=\Delta m_{j}^{0}\left(\tilde{m}_{j t}^{0}\right)$.

The coefficient $b_{1}$ on the change in care process (use of PHBs) is a function of the direct effect on outcome (difference), $\theta_{1}$, and the indirect effects of PHB-use on services $\left(\theta_{2} \mu_{1}\right)$ and dependency $\left(\theta_{3} \delta_{1}\right)$. Our assessment of personal health budgets would also benefit from an estimate of the direct effect only, mainly because the adoption of a personal health budget model need not mean a change in resources. The size of $\alpha_{1}$ is a policy choice. If sites used a pure substitution approach, for example, then this parameter would be zero. The complicating factor, however, is that $\mu_{1}$ need not be zero even if $\alpha_{1}=0$ because the use of personal health budgets could reduce need, $m_{j t}$, which in turn reduces the demand for services. One way we chose to proceed was to estimate the full structural model (C-1), which was done more easily in difference form i.e.:

$$
y_{j t+1}-y_{j t}=b_{0}^{S}+b_{1}^{S}\left(B_{j t+1}-B_{j t}\right)+b_{2}^{S}\left(x_{j t+1}-x_{j t}\right)+b_{3}\left(m_{j t+1}^{D}-m_{j t}^{D}\right)+\varepsilon^{S}
$$

where the $S$ superscript denotes coefficients from the structural model. In estimating this model in practice, we are never likely to have a complete set of NRC factors available. In this study, we used a subset of factors $m_{j t}^{D} \subset m_{j t}$, specifically: ADL need, informal care rates and income proxies. Unlike the choice of $B_{j t+1}$ which is made at baseline, $x_{j t+1}$ is determined after baseline (at time $t+1$ ) and it is a function of $m_{j t+1}^{D}$. As such the difference $\left(x_{j t+1}-x_{j t}\right)$ is likely to be correlated with omitted NRC change factors that appear in the error term, producing endogeneity bias. In other words, the coefficient $b_{2}^{\mathrm{S}}$ in an OLS estimation would be 
picking up some of the effects of changes in NRC. For example, other things equal, an increase in services would be expected to improve outcomes. But the demand for services is also dependent on the person's health state. Other things equal, if people's health deteriorates (for external reasons), their quality-of-life score would decrease, and at the same time, service use would increase. Where this effect is strong, omitting proxies for health state could lead to a negative correlation between change in outcomes and change in service use.

\section{C.3 Instrumental variables estimation}

We accounted for this problem by including as many changes in NRCs factors as possible and also by using instrumental variables estimation of (C-10). We estimated these models using both ASCOT and EQ-5D as outcome indicators. In both cases, the strategy for selecting instruments was to use baseline health state and care need indicators that were correlated with the change in total service expenditure but not with a change in outcomes. By including the change in these instruments directly in the model as explanatory factors the intention was to remove any correlation between their baseline value and the outcome change dependent variable (without removing the correlation between their baseline value and change in services). Baseline ADL score indicators were found to work well for ASCOT. For EQ-5D, because this measure is usually highly correlated with ADL levels, using ADL score indicators led to an over-identified model. Instead we opted to use time 1 psychological wellbeing (GHQ12) as an instrument in that the EQ-5D measure concentrates more on physical health. In addition, for EQ-5D we used our baseline income proxy - whether the person is in receipt of income benefits. Service utilisation, especially social care, tends to be influenced by income, but short-term changes in income are less likely to affect health directly (other than through service use). Over-identification was not indicated when benefits receipt at follow-up was also included in the model.

In working on the imputed dataset, we manually instrumented for $\Delta x_{j t}$ using a first-stage multiple imputation (MI) OLS (ordinary least squares) estimation of the change in total expenditure on the included and excluded instruments. The predicted values for each imputation were used in an MI OLS model as follows:

$$
\Delta y_{j t}=b_{0}^{S}+b_{1}^{S} \Delta B_{j t}+b_{2}^{S} \Delta \widehat{x}_{j t}\left(m_{j t}^{I}, \Delta m_{j t}^{D}, \Delta B_{j t}\right)+b_{3} \Delta m_{j t}^{D}+\varepsilon^{S}
$$

Instrument weakness was tested by an MI test of joint significance (of the instruments in the first-stage MI OLS model). Over-identification was tested using a Sargan style test with the mean R-squared values from an MI OLS estimation of the residuals $\varepsilon^{S}$. Finally, endogeneity was tested using a Hausman test by including the residual of the first-stage equation in an MI OLS estimation of the main model using the $\Delta x_{j t}$ rather than its predicted value. We were not able to correct the standard errors on the coefficient estimates in the main-stage MI model for the stochastic nature of $\widehat{\Delta x}_{j t}$ and therefore our significance estimators are subject to some (small) error. 
The model results are given in the main text. Overall, the estimation performed reasonably well although we were hampered by the usual problem of weak instruments. The instruments were jointly significant but the test statistic was a little lower than the usual benchmarks. On the other hand, the over-identification and endogeneity tests showed no further issues and supported our rejection of the exogeneity hypothesis.

We discuss the results in the main text.

\section{C.4 Difference-in-difference approach}

On the basis of the theoretical model and empirical strategy outlined above in C.1, we used a difference-in-difference (DiD) approach for the main analyses. In other words we calculated the difference between personal health budget and control groups in the change through time of the relevant indicator e.g. outcome scores such as EQ-5D, ASCOT, GHQ-12 and also costs and net effects (see below).

The difference-in-difference (DiD) estimator is calculated as:

$$
\Delta y=\left(\bar{y}_{11}-\bar{y}_{10}\right)-\left(\bar{y}_{01}-\bar{y}_{00}\right)
$$

where $\bar{y}_{i t}$ is the sample mean value of the intervention group $i$ subgroup at time $t$. Taking the whole sample, the DiD estimator is identical to the coefficient $b_{1}$ in the estimation of difference model (C-8).

This approach is also equivalent to fitting a pooled OLS model as follows:

$$
y_{j t}=\beta_{0}+\beta_{1} B_{j}+\beta_{2} T_{j t}+\beta_{3} B_{j} T_{j t}+e_{j t}
$$

where $B_{j}=0,1$ is a 'treatment dummy' variable for the $j$ 'th person i.e. having a personal health budget or using the convention process. A time dummy variable is $T_{t}=0,1$. The coefficient on the interaction term $X_{j} T_{t}$ is the DiD estimate: $\Delta y=\beta_{3}=b_{1}$.

As an alternative we can estimate a fixed effects ( $\mathrm{FE}$ ) model using a time variant indicator of personal health budget use, $B_{i t}$ :

$$
y_{j t}=\mu_{0}+\mu_{1} T_{j t}+\mu_{2} B_{j t}+c_{j}+e_{j t}
$$

The standard errors between the OLS and FE models will differ slightly (see Wooldridge, 2002).

We can use these approaches to estimate the impact on effectiveness, costs and also NMB (net monetary benefit). Consider the latter. At any given time $t$, the NMB for each person $j$ is:

$$
N M B_{j t}=\lambda E_{j t}-C_{j t}
$$

and the difference over time in NMB is: 


$$
\Delta^{t} N M B_{j}=\lambda\left(E_{j 1}-E_{j 0}\right)-\left(C_{j 1}-C_{j 0}\right)
$$

We can estimate NMB using a fixed-effects model as above (substituting $y_{j t}=N M B_{j t}$ in (C-14)).

\section{C.5 Mortality and confounding factors on outcome indicator changes}

A difference-in-difference approach controls for baseline differences in the level of the outcome indicator. There remains a possibility that baseline differences in characteristics between the groups might cause differences in the rate of change of the indicator, beyond any true effect of personal health budgets. We can control for this by estimating DiD models incorporating baseline characteristics:

$$
\Delta y_{j}=\beta_{0}+\beta_{1} m_{j 0}+\beta_{2} B_{j}+e_{j 1}
$$

where $m_{j 0}$ are baseline NR characteristics.

We might argue that outcome indicators such as wellbeing scores are less inherently susceptible to baseline differences causing spurious change effects 12 months later because the most important determinants at any given time are those more-immediate and everyday factors, including use of a personal health budget. This argument is much more difficult to sustain for mortality rates, especially because a lack of difference in mortality at baseline can hide substantial differences in the propensity to die immediately after baseline (where, clearly, dead people cannot be recruited into the study). It is therefore most important to control for baseline factors that affect change, as in (C-17).

It is also for this reason that we should note the limitations of baseline control estimations and consequently that the design of this study was not the ideal one for considering the mortality question. There is always the possibility that some significant factor is omitted so that, on average, people in one group had a higher propensity for mortality than those in the other group at baseline, regardless of the effects of personal health budgets. Ideally, an RCT would have been used to avoid this possibility.

\section{C.6 Costs}

People receiving PHBs used services and support funded from the personal health budget and also from conventional budgets. There is a wide range of forms of support or services that people can use, which we classify as, firstly, indirect services, namely: primary healthcare; secondary inpatient healthcare, outpatient and A\&E services; and secondly, direct services: nursing (mainstream) and therapy services; specialist nursing and other health services; social care, equipment and respite support; and well-being and leisure support. Personal health budgets can be used to secure all these services except the first three, the indirect services, but we measure the use of indirect services because the use of a PHB may change people's demand for these services. In theory, the use of a personal health budget might also affect 
demand for other (non-PHB) services such as prescription drugs, but measuring this wider impact was deemed to be beyond the scope of this study.

As a consequence of study participants potentially receiving support via their budgets and via conventional routes we needed to measure service use distinguishing these funding sources. Due to the difficulties of making this distinction in the data collection our approach was to measure, as far as possible, total use of services and support and also activity funded by the personal health budget as specified in the care and support plan. To this end, the study involved four methods to capture the use of this range of services: secondary care was captured using HES data; medical records were used to measure primary and AHP care; the service user interview was the vehicle to capture the use of nursing and social care services and the care and support plan was used to measure all services funded by the personal health budget.

The variety of implementation forms for personal health budgets in the study complicated the calculation of total costs of all services and support used by participants in the PHB and control groups. Our costing methodology is outlined in what follows.

We began by distinguishing five categories of service or support activity. These categories combined one or more specific activity, $x_{j t}^{k}$, of the $k=1$ to 11 services listed above (and in table 2-1 in chapter 2), for each participant $j$ at time $t=1,2$ (baseline and follow-up). Cost is calculated by multiplying the activity level by the corresponding unit cost, $c_{t}^{k}$ :

- Indirect service (secondary and primary) cost is: $I_{j t}^{N}=\sum_{k=1}^{2} c_{t}^{k} x_{j t}^{k}$.

- Specialist nursing and other health cost is:

$h_{j t}^{m}=c_{t}^{4} x_{j t}^{4}$

- Nursing and therapy services cost is:

$q_{j t}^{m}=c_{t}^{5} x_{j t}^{5}$

- Social care, equipment, respite cost is:

$s_{j t}^{m}=\sum_{k=6}^{9} c_{t}^{k} x_{j t}^{k}$

- Wellbeing and informal care pay cost is: $w_{j t}^{m}=c_{t}^{10} x_{j t}^{11}+c_{t}^{10} x_{j t}^{11}$

where $m=N, B$ for conventionally-funded and PHB-funded services respectively.

Total costs for study participants in group $j(j=1$ for PHB and $j=0$ for control) at time $t$ ( $t=1$ for baseline and $t=2$ for follow-up) were defined as:

$$
C_{j t}=I_{j t}^{N}\left(B_{j t}\right)+q_{j t}^{N}+q_{j t}^{B}+s_{j t}^{N}+s_{j t}^{B}+w_{j t}^{B}+h_{j t}^{N}+h_{j t}^{B}
$$

Our definition of well-being support and services was chosen to rule out any services that might be conventionally provided. We therefore defined $w_{j t}^{N}=0$ for all $j$ and $t$.

For the personal health budget group at time 2 , total cost, $C_{12}$ was calculated as:

$$
C_{12}=I_{12}^{N}\left(B_{12}\right)+q_{12}^{N}+q_{12}^{B}+s_{12}^{N}+s_{12}^{B}+w_{12}^{B}+h_{12}^{N}+h_{12}^{B}
$$

where the PHB funded $B_{12}=q_{12}^{B}+s_{12}^{B}+h_{12}^{B}+w_{12}^{B}+B_{12}^{0}$ with $B_{12}^{0}$ being any underspent part of the budget. The components of this equation were measured as follows. 
Indirect service use cannot be funded using a personal health budget and therefore our measurement of total primary and secondary care expenditure gives us the value $I_{12}^{N}$. We measured this expenditure for both personal health budget and control groups because we allow for the possibility that indirect service use is affected by whether or not a person has a PHB i.e $\frac{\partial I_{12}^{N}}{\partial B_{12}} \neq 0$.

As regards direct services, we also assumed that expenditure on the personal health budget substitutes in part for spending via conventional budgets. In particular we supposed that

$$
C_{12}^{N}=\bar{C}_{12}^{N}-f^{C}\left(C_{12}^{B}\right)
$$

for each direct service $C=q, s, h, w$ and where $\bar{C}_{12}^{N}$ is the service expenditure without a personal health budget. For example, social care expenditure may be shifted from conventional budgets to the personal health budget: $s_{12}^{N}=\bar{s}_{12}^{N}-f^{S}\left(s_{12}^{B}\right)$. In general a f1-forf1 substitution would be: $C_{12}^{N}=\bar{C}_{12}^{N}-C_{12}^{B}$ (or $s_{12}^{N}=\bar{s}_{12}^{N}-s_{12}^{B}$ in the social care case) so that a full transfer would result in: $C_{12}^{N}=0$ and $C_{12}^{B}=\bar{C}_{12}^{N}$.

By definition, total expenditure by service type for the personal health budget group was: $C_{12}^{T}=C_{12}^{N}+C_{12}^{B}$ for $C=q, s, h, w$. Ideally, in the study we aimed to collect data on the total use of all forms of service and support and also the amount funded out of the personal health budget i.e. to measure $C_{12}^{T}$ and $C_{12}^{B}$. In practice, the care plan gave us the breakdown of expenditure made from the personal health budget but there was no equivalent way of measuring the amount of each form of service or support that was funded conventionally. As outlined we could instead measure the total use of those services, where in theory the personal health budget could be used to fund part of this total alongside conventional budgets. There is a difficulty with this approach however. Respondents might not include all PHB-funded service/support category because they did not use their personal health budget according to conventional service definitions. For example, suppose we are measuring social care expenditure. A personal health budget holder who was receiving conventional home care services at baseline, at a value of $£ 250$ per week, instead uses their budget at follow-up to pay for a personal assistant. Their PA might cost the same per week ( $£ 250$ ), but could we be sure that the study participant would record total home care use at follow-up at $£ 250$ per week? The cost of PA support could be confirmed in the support plan however.

In this way we assumed that the observed total might not include all PHB-funded care: i.e. $\tilde{C}_{12}^{T}=C_{12}^{N}+g^{C} C_{12}^{B}$ where $\tilde{C}_{12}^{T}$ is the observed total such that the PHB contribution may be under-counted such that $0 \leq g^{C} \leq 1$. We can re-arrange in this function and substitute for $C_{12}^{N}$ i.e. $\tilde{C}_{12}^{T}-g^{C} C_{12}^{B}=C_{12}^{N}$. As such actual expenditure is:

$$
C_{12}^{T}=C_{12}^{N}+C_{12}^{B}=\left(\tilde{C}_{12}^{T}-g^{C} C_{12}^{B}\right)+C_{12}^{B}
$$

Clearly if all PHB-funded care was counted i.e. $g^{C}=1$ then $C_{12}^{T}=\tilde{C}_{12}^{T}$.

We therefore needed an estimate of $g^{C}$. For this purpose, we distinguished between two types of personal health budgets process. First there were sites that offered relatively small 
budgets (of less than $\mathrm{f1000}$ ) which were provided on top of conventional services, with funding from new sources. The second group of personal health budgets were those funded by a reduction of budgets for conventional services. These were generally more substantial. On this basis we assumed that personal health budgets with budgets of greater than $£ 1000$ in each service component are fully substituting, as outlined above i.e. $C_{12}^{N}=0$ with $f^{C}=1$. Those with budgets of less than $£ 1000$ were assumed to have personal health budgets funded in addition to usual services i.e. $C_{12}^{N}>0$ with $f^{C}=0$.

Using (C-20) we can write: $\tilde{C}_{12}^{T}-g^{C} C_{12}^{B}=C_{12}^{N}=\bar{C}_{12}^{N}-f^{C}\left(C_{12}^{B}\right)$. With full substitution i.e. $C_{12}^{N}=0$ then $\tilde{C}_{12}^{T}=g^{C} C_{12}^{B}$ and $(\mathrm{C}-21)$ reduces to

$$
C_{12}^{T}=C_{12}^{B}
$$

and under-counting is not a problem. But where there is partial substitution, under-counting might occur. In that case, actual expenditure is given by (C-21) and we need to make an assumption about the size of $g^{C} \leq 1$ since $C_{12}^{N}=\bar{C}_{12}^{N}-f^{C}\left(C_{12}^{B}\right)>0$. One way of doing this was to estimate a value of $g^{C}$ from the relationship $\tilde{C}_{12}^{T}=g^{C} C_{12}^{B}$.

We took a sub-sample of cases where budgets are greater than $£ 1000$, for which we assumed that $\tilde{C}_{12}^{T}=g^{C} C_{12}^{\bar{B}}$, and where both expenditure amounts were measured. Using simple regression we estimated the coefficient $g^{C}$. For social care services we estimated $g^{S}=0.47$ and for nursing and therapy services, $g^{q}=0.16$. These values were assumed to apply to budgets of less than $£ 1000$ where $C_{12}^{N}>0$ and actual total expenditure is given by (C-21). With the observed total expenditure $\tilde{C}_{12}^{T}$ from the interviews at follow-up and $C_{12}^{B}$ from support plans, and using these estimates we calculated total social care and nursing and therapy services. In the social care case we have:

- For $s_{12}^{B}>£ 1000$ we have: $s_{12}^{N}+s_{12}^{B}=s_{12}^{B}$

- For $s_{12}^{B} \leq £ 1000$ we have: $s_{12}^{N}+s_{12}^{B}=\tilde{s}_{12}^{T}-0.47 s_{12}^{B}+s_{12}^{B}$

Similarly for nursing and therapy services we have:

- For $q_{12}^{B}>£ 1000$ we have: $q_{12}^{N}+q_{12}^{B}=q_{12}^{B}$

- For $s_{12}^{B} \leq £ 1000$ we have: $q_{12}^{N}+q_{12}^{B}=\tilde{q}_{12}^{T}-0.16 q_{12}^{B}+q_{12}^{B}$

These amounts were substituted into (C-19).

We also assumed that the social care of any personal health budget might include elements from any existing social care personal budget (PB). Specifically we expected that the social care component in the PHB would be a function of the existence of time 1 social care PBs, including direct payments: $s_{12}^{B}=s_{12}^{B}\left(B, b_{11}\right)$, where $b_{11}$ is the existence of a social care personal budget at time 1.

Regarding well-being support and services in (C-19), as noted above, we defined $w_{12}^{N}=0$. Well-being support and services listed in the personal health budget support plan were used to cost $w_{12}^{B}$ in (C-19). 
The specialist nursing and other health services are somewhat of a catch-all category for health care services not captured in the other categories. As such we were not able to collect information about total activity in this category except for that listed in the personal health budget support plan. In other words, we assumed that $h_{12}^{N}=0{ }^{11}$. We made the same assumption for the control group so as to not bias the cost comparison.

For the personal health budget group at time 1:

$$
C_{11}=I_{11}^{N}+q_{11}^{N}+s_{11}^{N}+s_{11}^{B} b_{11}+w_{11}^{B} b_{11}+h_{11}^{N}
$$

Indirect services are measured in the same way as time 2 . In the absence of personal health budgets, conventionally-funded nursing and therapy support equals the total observed level: $q_{11}^{N}=\tilde{q}_{12}^{T}$.

As regards social care, and despite personal health budgets not being available, participants might have been receiving social care personal budgets $(\mathrm{PB})$ at time 1 . Interview data at baseline indicated whether a person had a social care PB, but we did not have access to PB care plans at time 1.

In theory the same social care services can be funded in a social care personal budget as those in a personal health budget (where they are often combined). We therefore estimated the cost of time 1 social care personal budgets using the cost of social care in the time 2 personal health budget and controlling for any changes in study participant characteristics, such as ADL dependency, and also baseline characteristics. Hence we use: $\hat{s}_{11}^{B}=\hat{s}_{12}^{B}\left(\delta_{11}\right)$ from the time 2 regression: $s_{12}^{B}=\hat{s}_{12}^{B}\left(\delta_{12}\right)+\varepsilon^{s}$. To avoid double-counting, we calculate $s_{11}^{N}+s_{11}^{B} b_{11}=\tilde{s}_{11}^{T}+$ $(1-0.47) \hat{s}_{11}^{B} b_{11}$ in all cases.

We can also make the similar argument that people with social care personal budgets might have been using that budget to secure wellbeing services. As with social care, we used the wellbeing component of the time 2 personal health budget as an indicator, controlling for any changes in characteristics: $w_{11}^{B}=\widehat{w}_{12}^{B}\left(\delta_{11}\right)$. As noted above, with personal health budgets of less than $f 1000$, we assumed that the personal health budget was provided on top of existing services and support and this could include existing time-1 social care personal budgets. In this case the actual value of the social care personal budget would be less than implied by the time-2 personal health budget. In this case, the social care personal budget would be less by $f 1000$ or the value of the personal health budget, whichever is greater (so that any time-1 personal budget is greater or equal to zero).

\footnotetext{
${ }^{11}$ Given the complexities of measuring the use of routine equipment and costing this use, particularly in terms of the opportunity cost where equipment is borrowed from an existing (slowly depreciating) stock (i.e. treated as capital), we assumed that this cost was also zero when funded from conventional budgets.
} 
Although we did not collect data on specialist and other health care that is conventionallyfunded, it is important not to count the amount in the personal health budget at follow-up as purely additional costs. Where personal health budgets were substituting for conventionally funded services, any expenditure on these services in the personal health budgets at time 2 will have been in part transferred from a conventional budget at time 1 . We could have proceeded by removing these costs from the total i.e. setting $h_{12}^{B}=0$ but this would have under-costed the personal health budget group because some sites made additional budget available and this was used to purchase specialist nursing and other health services. Instead, we opted to infer the value of these services when they were provided at time 1 in a conventional budget. This was done in the same way as inferring the time 1 social care personal budget i.e. $h_{11}^{N}=\hat{h}_{12}^{B}\left(\delta_{11}\right)$.

In either case, we have:

$$
\begin{aligned}
s_{11}^{B} b_{11}+w_{11}^{B} b_{11} & +h_{11}^{N} \\
= & \left(\hat{s}_{12}^{B}+\widehat{w}_{11}^{B}\right) b_{11}+\hat{h}_{11}^{B}-\left(\Delta^{E} s_{12}^{B}+\Delta^{E} w_{12}^{B}+\Delta^{E} h_{12}^{B}\right) \\
& -\left(\Delta^{T} s_{12}^{B}+\Delta^{T} w_{12}^{B}+\Delta^{T} h_{12}^{B}\right)
\end{aligned}
$$

where $\Delta^{E}$ signifies the extra money available in the personal health budget compared to time 1 that comes on top of conventional funding. It is also possible that people transfer expenditure at time 2 from their budget between the different service components (compared to the distribution in their time 1 personal budget). This transfer could be positive or negative but we have no basis for calculating this value. We therefore assume the net transfer is zero i.e. $\left(\Delta^{T} s_{12}^{B}+\Delta^{T} w_{12}^{B}+\Delta^{T} h_{12}^{B}\right)=0$. As noted the extra amount $\Delta^{E}$ is assumed to be zero for personal health budgets over $£ 1000$, but will be up to the value of the PHB for budgets of less than $f 1000$. In other words, when substituting (C-24) into (C-23) we use $s_{11}^{B} b_{11}+w_{11}^{B} b_{11}+$ $h_{11}^{N}=\left(\hat{s}_{12}^{B}+\widehat{w}_{11}^{B}\right) b_{11}+\hat{h}_{11}^{B}-B_{12}$ or $s_{11}^{B} b_{11}+w_{11}^{B} b_{11}+h_{11}^{N}=0$ whichever is greater.

For the control group, overall cost per individual is:

$$
\begin{aligned}
C_{0 t}=I_{0 t}^{N}+q_{0 t}^{N} & +s_{0 t}^{N}+\left(s_{0 t}^{B}+w_{0 t}^{B}\right) b_{11} \\
& =I_{0 t}^{N}+\tilde{q}_{0 t}^{T}+\tilde{s}_{0 t}^{T}+\left(\hat{s}_{0 t}^{B}(1-0.47)+\widehat{w}_{0 t}^{B}\right) b_{11}
\end{aligned}
$$

that is, we use the observed totals for each service component in the same way as for the personal health budget group. We also include the costs of social care personal budgets in the control group (again accounting for possible double counting) using the same predicted values as for the personal health budget group but with control group characteristics. Costs are calculated in the same way for time 1 and time 2 .

\section{C.6.1.1 Secondary care use}

We have both administrative data (from the Hospital Episode Statistics or HES) and self-report data. In the main we aim to use HES data over self-report because the latter can embody recall bias. The Payment by Results tariff reimburses inpatient activity with an admission payment and a long-stay adjuster and therefore average unit cost (per bed day) is not constant. We use 
the admitted patient tariff to cost this activity, and write the cost for patient $j$ as $c_{j t}^{1}\left(x_{j m}^{1}\right) x_{j m}^{1}$, where $\frac{\partial c_{j t}^{1}}{\partial x_{j m}^{1}}<0$.

Due to the timing of the study the latest available HES data were for the year ending 31 March 2012. As a result of the late up-surge in study recruitment around half the people in the sample have a follow-up date after 31 March 2012, with the last follow-up interviews done at the end of July 2012. We therefore lack HES data for between one to four months at the end of the follow-up period for these cases. We can, however, use self-reported data where HES data is missing. Suppose monthly HES costs were $c_{t}^{1}\left(x_{j m}^{1}\right) x_{j m}^{1}$, where the subscript $m$ denotes 31 March in the month after baseline. Then the sum of these costs over the period (in months) of month $m$ to month $M$ is $C_{j m}^{M}$, which is,

$$
\sum_{m}^{M} c_{t}^{1}\left(x_{j m}^{1}\right) x_{j m}^{1}=C_{j m}^{M}
$$

We estimated inpatient costs for missing months as follows. First, we estimated 4 models, for each of the last 4 months:

$$
C_{j m-1}^{m}=C_{j m-1}^{m}\left(C_{j 1}^{m-1}, V_{j}, V_{j} C_{j 1}^{m-1}, G_{j}, \delta_{j}\right), \quad m=9,10,11,12
$$

where

$V$ is self-reported visits over the year

$G$ is personal health budget or control group

$\delta$ is person characteristics

The sample in each case was all participants excluding those with month $m$ missing. For the missing cases, the predicted values were used for hospital costs in the corresponding missing month. A zero-inflated negative binomial model was used for the estimations.

\section{C.6.1.2 Primary care use}

We have two sources of data on primary care use: the medical record extracts and the main interviews, both at baseline and at 12-month follow-up. We collect information on surgery and home GP visits and on practice/community nurse visits using both these sources. To minimise recall bias we ask interviewees about service use in the last one month. From medical records we extracted the last year's worth of service use. In terms of priority we used the medical records information as the primary source and turned to the self-report data where the former was missing. In order to reconcile the different time periods in these collections we first estimated annual primary care use from baseline medical records using self-report data as a predictor. Given the significant proportion of zero-use across the sample in this count data, we estimated the model using a zero-inflated negative binomial model: 


$$
y_{j 0}^{m}=p^{m 0}\left(y_{j 0}^{s}\right) y_{j 0}^{1 m}\left(y_{j 0}^{s}\right)+\epsilon_{j}
$$

where

$y_{j 0}^{m}$ is annual primary care use from medical records (person $j$ at time $t=0$ )

$y_{j 0}^{S}$ is the previous month's primary care use from the interview

$p^{m 0}$ is the probability of zero annual use

$y_{j 0}^{1 m}$ is the level of annual use conditional on a person having at least one visit

We then used the time 0 (baseline) estimation results to convert self-reported service use in the last month (at time $t$ ) to annual use where the latter was missing at either baseline (time $t$ $=0$ ) or at following up (time $t=1$ ):

$$
\hat{y}_{j t}^{m}=\left(1-\hat{p}^{m 0}\left(y_{j t}^{s} ; \beta_{t=0}^{0}\right)\right) \exp \left(\hat{y}_{j t}^{1 m}\left(y_{j t}^{s} ; \beta_{t=0}^{1}\right)\right)
$$

We set $y_{k t}^{m}=\hat{y}_{k t}^{m}$ for each $j=k$ missing values.

\section{C.7 Carers}

A sub-sample of main study participants consented for the research team to contact their carer at time 1. We collected information on carer outcomes, including EQ-5D, ASCOT and GHQ-12, which we denote, respectively, $y_{k 1}^{c}$. We also collected some information on their time-invariant characteristics, such as sex, as well as factors that we assume are age-invariant or predictable, such as housing tenure, living arrangements and age group. These are denoted $z_{k}$ below. Caring-related outcomes that are potentially affected by personal health budgets are also likely to be influenced by the characteristics of the cared-for person. To this end, we included relevant baseline (TO) needs-related characteristics and also the baseline quality of life indicators for the cared-for person, denoted respectively, $z_{k 0}^{j}$ and $y_{k 0}^{j}$.

Carer outcomes were then estimated by the following model:

$$
y_{k 1}^{c}=\beta_{0}^{c}+\beta_{1}^{c} z_{k 0}^{j}+\beta_{2}^{c} y_{k 0}^{j}+\beta_{3}^{c} z_{k}+\beta_{4}^{c} X_{i}+e_{i 1}
$$

An OLS (ordinary least squares) estimator was used. As with the mortality analysis, omitted variable problems, especially relating to the (unobserved) baseline quality of life characteristics of carers, could apply. Given this issue and the relatively small sample, we need to be somewhat cautious when interpreting the results.

\section{C.8 Missing values and multiple imputation}

Multiple imputation (MI) was used to deal with missing data according to Rubin's method (Rubin, 1987). MI requires that the missing values in the data are missing at random (MAR) 
(StataCorp, 2011). Missing data are missing at random (MAR) if the probability that the data are missing does not depend on unobserved data, but may depend on observed data (StataCorp, 2011). Under MAR, the missing-data values do not contain any additional information given observed data about the missing-data mechanism. We can distinguish MAR from two other types of missing data patterns. Missing completely at random (MCAR) is where the probability that data are missing does not depend on observed or unobserved data. Missing not at random (MNAR) is where part of the reason that the data are missing is explained by variables that have missing values.

We can use MI for MAR (and MCAR) but not with MNAR. Essentially, MI requires that the reasons/mechanism for the data being missing must be accounted for in the model (i.e. by factors that do not have missing values). The alternative strategy of dropping cases with missing data is generally only valid for MCAR data, and could lead to bias for MAR and MNAR data. The options available for MNAR are limited to (a) assuming a priori, a mechanism for predicting missing data and configuring the imputation process on that basis, or (b) finding additional (non-missing) data that can be reasonably argued to reflect the reasons why these data were missing. In both cases, some form of sensitivity analysis - where the data are imputed on different configurations - is recommended.

What are the implications for the evaluation of personal health budgets? We categorised the following types of missing data in the study:

- People overlooking or discarding individual questions. These data are either MCAR (missing completely at random) or it could be MAR (missing at random). In this MAR case, people's baseline characteristics such as their level of frailty, which are measured, can explain non-response.

- Withdrawal of consent for external reasons. One example would be a change of family circumstances outside the study such as the person moving away as the cause of missing data. These are MCAR (missing completely at random) missing values and could be either dropped or imputed.

- Loss to follow-up due to severe frailty, ill health or poor quality of life of the study participant i.e. no follow-up interview data. There were two relevant considerations. Where we could argue that the cause of severe ill health was unrelated to the study directly, i.e. that having a personal health budget does not affect severe morbidity, then this missing data is MCAR (missing completely at random). However, if the treatment choice potentially does have an impact on morbidity as we expect, then the issue is: (a) whether the missing data were direct causes of the drop-out - and so MNAR (missing not at random), or (b) whether the missing data are consequences of the intervention and other non-missing characteristics, which are observed, and so MAR (missing at random). We maintain that the latter case seems a more reasonable judgement and that we could impute these missing values. 
Study consent was initially gained from 2,700 people. Some 302 people were excluded from the study because they had neither taken part in the baseline or follow-up interviews (and had in effect withdrawn consent before baseline), because they were in residential care at baseline, or because they had died before baseline. The remaining 2,398 cases were suitable for multiple imputation. Of these, 158 people died before follow-up. A further 5 cases were dropped because they were aged less than 18 (these cases were dropped after the imputation because some ages were missing in the MI sample of 2398). For the main analysis we dropped the 158 people that had died because we expected them to have a relatively different profile of costs and benefits than people that were still alive at follow-up. Although these data are potentially missing not at random (MNAR), they were a small number of cases. We also found no significant differences in mortality rates between the personal health budget and control groups and so were content to drop these cases. This left an active sample for the analysis of 2235 cases.

The pattern of missing data in the sample was as follows. Regarding outcomes data from the EQ-5D, ASCOT, and GHQ-12 scores, we had at least some follow-up outcomes data in 1,656 cases ( $74.1 \%$ of the active sample of 2,235 cases). For services we had 2,104 cases (94.1\%) with at least some service data at follow-up. We had either some follow-up outcomes data or some service data for 2,133 cases (95.4\%), leaving $4.6 \%$ of cases with just baseline data (35 control and 67 personal health budget). For information on receipt of informal care by respondents we obtained data from 1,340 participants (60.0\%).

Reflecting the arbitrary (non-monotonic) pattern of missing data, multiple imputations of the dataset were constructed using the chained equation method (ICE). The imputation model was constructed with equation estimators in the following categories. We used:

- First, regression models without constraints for continuous and non-censored variables such as age, dependency score (a 36 point scale) and some outcome scores such as GHQ-12;

- Second, censored regression, conditional on health condition, for clinical outcomes;

- Third, censored regression with an upper limit of 1 for EQ-5D and ASCOT;

- Fourth, logit models for binary dummy variables such as sex, marital status, use of benefits and so on (these logit models were estimated with augmentation);

- Fifth, logit models for whether or not people used particular services (estimated without augmentation);

- Sixth, censored regression models (with a zero lower limit) for service cost as conditional on binary service use (service costs were transformed using Box-Cox with a power of 0.67 ). 
The imputation model therefore incorporated two-part models for service use with Box-Cox transforms in order to better estimate missing service costs. A variety of final model specifications were used and in some cases specific variables were omitted to ensure convergence of the imputation run. Following Rubin (1987) the base model used 5 imputations (10 iterations). ${ }^{12}$ In total 152 variables were imputed in the dataset.

We conducted sensitivity analysis regarding the imputed dataset by generating a further 5 more imputations using a different random seed and adding these to the original 5 imputations. As outlined in chapter 6 , re-performing the analyses using this alternative dataset did not change the nature of the results.

\section{C.9 Uncertainty}

The estimates (including the difference-in-difference) are subject to statistical error. We used two methods to estimate the standard errors associated with the parameters, as follows.

\section{C.9.1 Non-parametric}

Standard errors were estimated using bootstrapping (1000 reps) for each of the 5 imputations of the data. We used the bootstrap (BS) mean and the bootstrap standard error for the 5 imputations to calculate overall mean, and BS standard error for the sample using Rubin's rules (Rubin, 1987; Schafer, 1997) (see Box C-1).

In this way we calculated BS Cls for the difference (over time) in effect $\Delta^{t} E_{i}$, the difference in cost $\Delta^{t} C_{i}$ and the difference in NMB $\Delta^{t} B_{i}$. We calculated each of these differences for both the intervention and control groups, bootstrapping each group separately. The DiD estimate was found by subtracting the control group difference from the personal health budget group difference. For example, the NMB difference for group $i$ is:

$$
\Delta^{t} N M B_{i}=\lambda\left(E_{i 1}-E_{i 0}\right)-\left(C_{i 1}-C_{i 0}\right)
$$

where $E_{i j}$ is the sub-sample mean value of effectiveness for intervention group $i$ and time $t$. Similarly, $C_{i j}$ is the cost. The DiD estimate is therefore:

$$
\Delta^{i} \Delta^{t} N M B=\Delta^{t} N M B_{1}-\Delta^{t} N M B_{0}
$$

The personal health budget and control groups as independent samples (each with clustering by site) for the purpose of finding BS standard errors. As such, the standard error of the DiD

\footnotetext{
${ }^{12}$ Five imputations may be regarded as at the lower limit, but was chosen in part for pragmatic reason in that the imputation procedure required over 7,500 model estimations to produce a dataset, the bulk of which were maximum likelihood models.
} 
estimate $\Delta^{i} \Delta^{t} y$ for $y=N M B, E, C$ is the sum of the BS standard errors for the estimates for each group i.e. $\hat{\sigma}\left(\Delta^{i} \Delta^{t} y\right)=\hat{\sigma}_{1}\left(\Delta^{t} y_{1}\right)+\hat{\sigma}_{0}\left(\Delta^{t} y_{0}\right)$ where $\hat{\sigma}($.$) is the total bootstrap standard$ error from the 5 imputations.

We can calculate the probability that $\Delta^{i} \Delta^{t} N M B(\lambda)>0$ for each $\lambda$. This can be done nonparametrically by finding the proportion of the $1000 \mathrm{M}$ repetitions where $\Delta^{i} \Delta^{t} N M B(\lambda)>0$ for a range of threshold values $\lambda$.

\section{Box C-1. Rubin's Rules}

Following the notation used in the text, the overall estimate is the average of the estimates from the individual imputations (of which we assume $M=5$ ):

$$
\bar{y}_{i}=\frac{1}{M} \sum_{m=1}^{M} y_{i m}
$$

Within-imputation variance:

$$
\overline{\hat{\sigma}_{l}^{W^{2}}}=\frac{1}{M} \sum_{m=1}^{M} \hat{\sigma}_{i m}^{2}
$$

Between-imputation variance:

$$
\hat{\sigma}_{i}^{B^{2}}=\frac{1}{M-1} \sum_{m=1}^{M}\left(y_{i m}-\bar{y}_{l}\right)^{2}
$$

Total variance is:

$$
\hat{\sigma}_{i}^{2}=\overline{\hat{\sigma}_{l}^{W^{2}}}+\left(1+\frac{1}{M}\right) \hat{\sigma}_{i}^{B^{2}}
$$

and so total standard error $\hat{\sigma}_{i}=\sqrt{\hat{\sigma}_{i}^{2}}$. This is distributed as a t-distribution with degrees of freedom of: $d f=(M-1)\left(1+\frac{M \overline{\widehat{\sigma}_{l}^{W^{2}}}}{(M+1) \widehat{\sigma}_{i}^{B^{2}}}\right)^{2}$.

\section{C.9.2 Parametric approach}

Estimating a fixed-effects model as in (C-14) or (C-8) using NMB as the dependent variable i.e.

$$
\Delta^{t} N M B_{j}=b_{0}+b_{1} \Delta^{t} B_{j}+e_{j}
$$

gives the difference-in-difference estimator as: $\Delta^{i} \Delta^{t} B=\mu_{2}$. We can estimate this model over 5 imputations of the data and combine estimates on each, including $\mu_{2}$, using Rubin's rules. Standard errors for each coefficient are estimated in the model on the usual assumption of a tdistribution. The system can be re-estimated for a range of $\lambda$ threshold values. For each $\lambda$ we 
can calculate the probability $\alpha$ required such that $\mu_{2} \equiv \Delta^{i} \Delta^{t} B>0$ i.e. that $\mu_{2}(\lambda)-t_{\alpha} \hat{\sigma}(\lambda)>$ 0 .

\section{Appendix D Examples of quotes from organisational representatives following PHB implementation models}

Table D-1. Quotes from organisational representatives implementing PHB model 1

\begin{tabular}{ll}
\hline $\begin{array}{l}\text { Organisational } \\
\text { representative }\end{array}$ & Quotes \\
\hline Project Lead & $\begin{array}{l}\text { Well firstly because of direct payments we've got a very good working relationship } \\
\text { with [the local council], we're really building on the integrated working, so we've used } \\
\text { [the local councils] finance systems to deliver the direct payments, and we've used all } \\
\text { of the policies and procedures to do that. }\end{array}$ \\
& $\begin{array}{l}\text { The reason people want to go on to a personal health budget is that while agencies } \\
\text { are very good at providing care, they do not provide consistency. For example, } \\
\text { someone might be having four visits a day, and within those four visits they have four } \\
\text { different people, and those four different people may be different on the next day, so } \\
\text { there's no consistency. }\end{array}$
\end{tabular}

Project Lead It's just taking way too long. I mean, there are people who were recruited to this pilot way back with our predecessor, so possibly on the pilot a year, and they still do not a budget. We do have some people who do not have an indicative budget because it's taking such a long time to get the costs revealed.

Health Professional It's been successful where you've got somebody with charisma, drive passion, with a real heart for it, and that personality has made it happen here. And I think we are very fortunate, we've got [project lead's name] who is just absolutely super, a real role model I think for leading it. I think other areas, who haven't got the same personality driving it haven't been quite as successful.

\begin{tabular}{|c|c|}
\hline $\begin{array}{l}\text { Third party provider } \\
\text { / budget holder }\end{array}$ & $\begin{array}{l}\text { They [PHBs] give people choice. As an individual, you do sometimes feel you are being } \\
\text { wheeled along a path whether you like it or not. So I am all for giving people the } \\
\text { opportunity to make decisions for themselves, but also people must take } \\
\text { responsibility for their decisions, if you make a wrong one you have to live with it. } \\
\text { Whoever makes the decision needs to take the responsibility. There is no point giving } \\
\text { people the chance of making decisions if they are not going to have some form of } \\
\text { responsibility for that decision. You can't blame someone else once you have decided } \\
\text { you want to do something. }\end{array}$ \\
\hline Service Provider & $\begin{array}{l}\text { It was okay to be able to explain what a personal health budget was, but nobody could } \\
\text { give any practical examples that were meaningful for our services and our clinicians to } \\
\text { really understand what it meant. So I think the information was limited. }\end{array}$ \\
\hline
\end{tabular}




\section{Organisational Quotes \\ representative}

Project Lead We've had some really good stories and we've had some really positive results of it, but equally there are some people who it's not really worked for and we've probably been giving people more things that we would do normally.

Third party provider The main thing l've seen, concerns about health and safety. Obviously you have / holder to take into account their health and safety and even giving somebody the knowledge of what risk factors there are in their choice......you're still not able to go with certain things, even if they feel that they can take that risk.

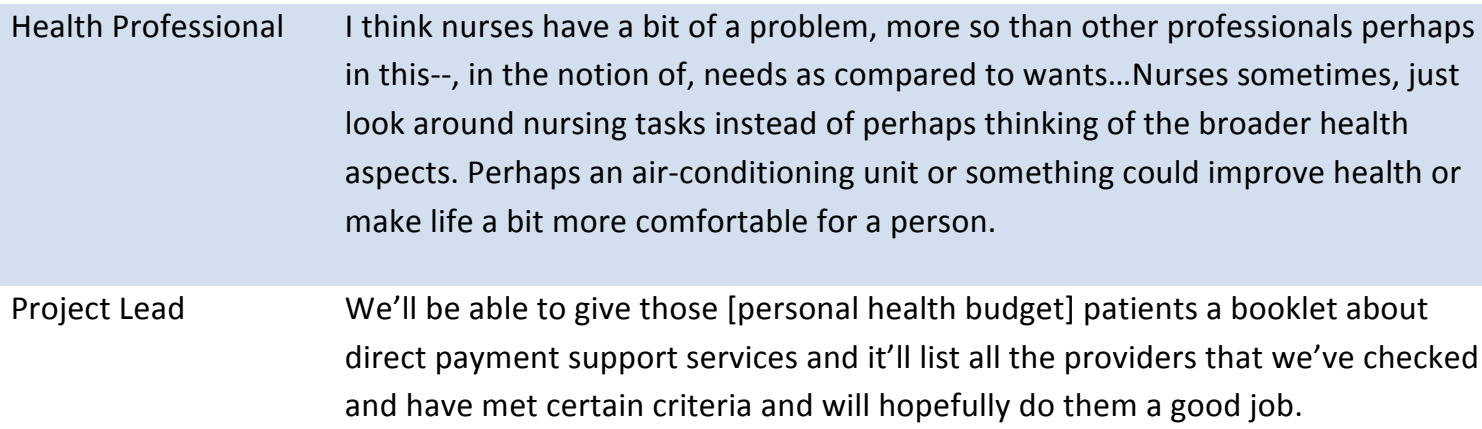
in this--, in the notion of, needs as compared to wants...Nurses sometimes, just look around nursing tasks instead of perhaps thinking of the broader health aspects. Perhaps an air-conditioning unit or something could improve health or make life a bit more comfortable for a person.

Project Lead We'll be able to give those [personal health budget] patients a booklet about direct payment support services and it'll list all the providers that we've checked and have met certain criteria and will hopefully do them a good job.

Project Lead Clinician views and opinions, that's been a massive block really because we've got a few clinicians who really don't like the idea and they sort of scupper all the plans that you try and put in place. So when other clinicians are talking about patients they think might benefit from a personal health budget, there's always a reason why it's not possible.

Third party provider / holder

I think there is an extensive list and I think that the more that they're done, the more personal budgets are available to people, the larger the list will become and the more knowledge that the brokers have...But I do think it's always an open document that can be added to.

Project Lead The disadvantages... it takes a long time to set up those things for the patients. So where a patient's only got one-off budget of say 300 pounds, there's no point in them receiving that as a direct payment. So we've had to organise them as a notional budget and we've had to organise the services on the patient's behalf, so obviously that takes a while and takes time and effort I suppose. 


\begin{tabular}{|c|c|}
\hline $\begin{array}{l}\text { Organisational } \\
\text { representative }\end{array}$ & Quotes \\
\hline Project Lead & $\begin{array}{l}\text { On an individual level, personal health budget have been a great success. The } \\
\text { individuals have benefitted greatly and I think more than anticipated for such a } \\
\text { relatively small amount of money compared to some of their packages that they } \\
\text { were receiving. We've started to see the personal health budgets have actually } \\
\text { given people more choice about how they would spend some money in a } \\
\text { different way from what traditional models would suggest. }\end{array}$ \\
\hline $\begin{array}{l}\text { Commissioning } \\
\text { Manager }\end{array}$ & $\begin{array}{l}\text { What we've discovered is that outside of primary care systems, not much money } \\
\text { gets spent on those people. So there isn't much money to divert into personal } \\
\text { health budgets and we've just been double spending. The general perception I } \\
\text { think of senior staff, and particularly of GPs, is that it's not worth the time and } \\
\text { effort. So, they will always fall back on clinical risk as an issue. }\end{array}$ \\
\hline $\begin{array}{l}\text { Commissioning } \\
\text { Manager }\end{array}$ & $\begin{array}{l}\text { I think it's been successful in getting people to be more engaged in their own } \\
\text { health. I think people enjoy the experience by and large, they take a bit of } \\
\text { persuading to get involved, quite a lot of them. They're sort of suspicious to start } \\
\text { with, but then I think they enjoy it. I think they enjoy making choices, I think it } \\
\text { does make them think more about their own health, so I thinks it's generally } \\
\text { very positive. }\end{array}$ \\
\hline Operational Staff & $\begin{array}{l}\text { To be honest when I've spoken to other people within the health service about } \\
\text { someone on the PHB and have received a generally negative attitude towards it. } \\
\text { The people who are negative towards it have come from a lot of different } \\
\text { camps. I would say that there isn't one particular group that has more of a } \\
\text { dislike for it than any other. }\end{array}$ \\
\hline Health Professional & $\begin{array}{l}\text { We work in an area of very high deprivation, with high mental health needs and } \\
\text { so on. So I think people being able to access the alternative therapy type } \\
\text { complimentary services has been very beneficial because they've been able to } \\
\text { access things that traditionally other people would pay privately for. And I think } \\
\text { they've found those services very beneficial and felt very lucky in a way. }\end{array}$ \\
\hline
\end{tabular}




\section{Organisational Quotes \\ representative}

Project Lead When people have come to us around thinking about personal health budgets, they generally have an idea about what it is that's going to make a difference, and so they've got quite a clear idea around outcome. Sometimes they know exactly where they can maybe purchase or, you know, kind of broker that either piece of equipment or service from.

Third party provider I think having to make health service staff think about the actual cost of their / budget holder services per head has been quite a challenge. I think having to realise that the disabled person or the patient might be the expert over them in some areas has been a real challenge but then again, a very positive one.

Operational Staff Some things have been quite straightforward.....spot purchase type things. Some of it's been a little bit more complex to organise, but then the outcomes have been good, so that's been worth investing in really.

Project Lead Speaking to somebody else who has been using direct payments for a while, also in fairly difficult circumstances, went some way to reassuring the family that, with the right support we can do this. And they have, they are successfully now using direct payments and are employing their own staff, with an understanding that it's new for them and it's new for us and there are things that we're learning that we may have to change and adapt as we go along.

Health Professional Through my own experience.....people are generally not aware of what statutory services are available to them and the health and social care makeup is currently so complex. People have to navigate themselves around systems in ways that maybe aren't necessarily meaningful to them, and very often I think some of the staff that are working within those organisations don't understand the full range of possibilities that are available to people.

Operational Staff I think staff are quite frightened about risk. I think staff are becoming, quite afraid to, you know, make changes to, as regards to, personal health budgets, and they're often quite reluctant to explore other ideas.

Project Lead

We're developing some kind of improved local knowledge as we go along around providers that we've used and know and trust and have got the appropriate qualifications, liability insurances and right approach. 


\section{Appendix E Questionnaires and instruments}

\section{List of questionnaires and instruments}

Baseline outcome questionnaire

Baseline medical record template

Follow-up outcome questionnaire - PHB Group

Follow-up outcome questionnaire - Control Group

Follow-up medical record template

Carer outcome questionnaire - Control Group

Carer outcome questionnaire - PHB Group

Month 3 budget holder topic guide

Month 9 budget holder topic guide

Month 3 topic guide - Project Leads

Month 3 topic guide - Operational staff

Month 3 topic guide - Health Professionals

Month 3 topic guide - Commissioning Managers

Month 3 topic guide $-3^{\text {rd }}$ party budget holders

Month 15 topic guide - Project Leads

Month 15 topic guide - Operational staff

Month 15 topic guide - Health Professionals

Month 15 topic guide $-3^{\text {rd }}$ party budget holders

Month 15 topic guide - Service providers

Month 15 topic guide - managers of user and carer organisations

Set-up cost template

These documents are provided after the following Appendix reference section. 


\section{Appendix references}

Bowling, A. 1995. Measuring Disease. Buckingham: Open University Press.

Bowling, A., Banister, D., Sutton, S., Evans, O. \& Windsor. 2002. A multidimensional model of the quality of life in older age. Aging and Mental Health, 6, 355-371.

Burge, P., Gallo, F. \& Netten, A. 2006. Valuing PSS Outputs and Qaulity Changes. PSSRU Discussion paper No 2356. Canterbury: Personal Services research Unit, University of Kent.

Curtis, L. 2010. Unit Costs of Health \& Social Care, 2010. Personal Social Services Research Unit, University of Kent.

Curtis, L. 2011. Unit Costs of Health \& Social Care, 2011. Personal Social Services Research Unit, University of Kent.

Dolan, P., Layard, R. \& Metcalfe, R. 2010. Measuring subjective wellbeing for public policy: Recommendations on measures. . London: The London School of Economics and Polical Science.

Ferraro, K. 1980. Self-ratings of health among the old and the old-old. Journal of Health and Social Behaviour, 21, 377-383.

Goldberg, D. 1992. General Health Questionnaire. Windsor: NFER Nelson.

Idler, E. \& Benyamini, Y. 1997. Self-rated health and mortality: a review of twentyseven community studies. Journal of Health and Social Behaviour, 38, 21-37.

Jones, K., Netten, A., Francis, J. \& Bebbington, A. 2007. Using older home care user experiences in performance monitoring. Health \& Social Care in the Community, 15, 322-332.

Malley, J., Sandhu, S. \& Netten, A. 2006. Younger adults' understanding of questions for a service user experience survey: A report to the Information Centre for Health and Social Care. Discussion Paper No. 2360. Canterbury: Personal Social Services Research Unit. University of Kent.

Robine, J. M., Jagger, C. \& Romieu, I. 2003. Selection of a coherent set of health indicators for the European Union. Phase 11: final report. Montpelier: EuroREVES.

Rubin, D. B. 1987. Multiple imputation for nonresponses in surveys, New Jersey, Wiley. Schafer, J. L. 1997. Analysis of incomplete multivariate data, London, Chapman \& Hall. Shadbolt, B. 2007. Some correlates of self-rated health for Australian women. American Journal of Public Health, 87, 951-956.

Statacorp. 2011. Stata statistical software: Release 11. College Station [Online]. TX: StataCorp LP. [Accessed].

Wooldridge, J. M. 2002. Econometric analysis of cross section and panel data, Cambridge, MA, MIT Press. 